\title{
GROTHENDIECK'S PAIRING ON NÉRON COMPONENT GROUPS: GALOIS DESCENT FROM THE SEMISTABLE CASE
}

\author{
TAKASHI SUZUKI
}

\begin{abstract}
In our previous study of duality for complete discrete valuation fields with perfect residue field, we treated coefficients in finite flat group schemes. In this paper, we treat abelian varieties. This in particular implies Grothendieck's conjecture on the perfectness of his pairing between the Néron component groups of an abelian variety and its dual. The point is that our formulation is well-suited with Galois descent. From the known case of semistable abelian varieties, we deduce the perfectness in full generality. We also treat coefficients in tori and, more generally, 1-motives.
\end{abstract}

\section{Contents}

1. Introduction

1.1. Main results

1.2. General picture

1.3. Organization

2. Site-theoretic preliminaries

2.1. Sites and algebraic groups: setup and first properties

2.2. Generalities on derived categories of ind-procategories

2.3. The derived categories of ind-proalgebraic groups and of sheaves

2.4. Serre duality and P-acyclicity

3. Local fields with ind-rational base

3.1. Basic notions and properties

3.2. Topology on rational points of varieties

3.3. The relative fppf site of a local field

3.4. Cohomology as sheaves on the residue field

4. Statement of the duality theorem

4.1. Formulation

4.2. Reduction to components groups and the first cohomology

5. Relation to Grothendieck's and Šafarevič's conjectures

5.1. Grothendieck's pairing

5.2. Bester-Bertapelle's isomorphism

5.2.1. Bester's finite flat duality

5.3. Bégueri's isomorphism 74

6. Galois descent

Date: March 22, 2018.

2010 Mathematics Subject Classification. Primary: 11G10; Secondary: 11S25, 14F20.

Key words and phrases. Abelian varieties; duality; Grothendieck topologies. 
7. End of proof: Grothendieck's conjecture 82

8. Duality with coefficients in tori 83

9. Duality with coefficients in 1-motives 90

10. Connection with classical statements for finite residue fields 92

Appendix A. The pro-fppf topology for proalgebraic proschemes 97

References 102

\section{INTRODUCTION}

1.1. Main results. Let $K$ be a complete discrete valuation field with ring of integers $\mathcal{O}_{K}$ and perfect residue field $k$ of characteristic $p>0$. Let $A$ be an abelian variety over $K, \mathcal{A}$ the Néron model of $A$ over $\mathcal{O}_{K}$ and $\mathcal{A}_{x}$ the special fiber of $\mathcal{A}$ over $x=\operatorname{Spec} k$. The component group $\pi_{0}\left(\mathcal{A}_{x}\right)$ of $\mathcal{A}_{x}$ is a finite étale group scheme over $k$. From the dual abelian variety $A^{\vee}$, we have corresponding objects $\mathcal{A}^{\vee}, \mathcal{A}_{x}^{\vee}$ and $\pi_{0}\left(\mathcal{A}_{x}^{\vee}\right)$. In Gro72, IX, 1.2.1], Grothendieck constructed a canonical pairing

$$
\pi_{0}\left(\mathcal{A}_{x}^{\vee}\right) \times \pi_{0}\left(\mathcal{A}_{x}\right) \rightarrow \mathbb{Q} / \mathbb{Z}
$$

which appears as the obstruction to extending the Poincaré biextension on $A^{\vee} \times A$ to the Néron models $\mathcal{A}^{\vee} \times \mathcal{A}$. In this paper, we prove the following conjecture of Grothendieck [Gro72, IX, Conj. 1.3]:

Grothendieck's conjecture. The pairing $\pi_{0}\left(\mathcal{A}_{x}^{\vee}\right) \times \pi_{0}\left(\mathcal{A}_{x}\right) \rightarrow \mathbb{Q} / \mathbb{Z}$ above is perfect.

Some previously known cases include: $k$ is finite $\mathrm{McC} 86$; $K$ has mixed characteristic Bég81; $A$ has semistable reduction Wer97; the prime-to- $p$ part Ber01; $A$ is the Jacobian of a curve with a rational point [BL02]; $A$ has potentially multiplicative reduction [Bos97, 1 McCallum used (the usual) local class field theory and local Tate duality. Bégueri instead used Serre's local class field theory Ser61, which is applicable for general perfect residue fields $k$. To deduce Grothendieck's conjecture, Bégueri used some dimension counting argument, which does not work in equal characteristic since the corresponding objects are infinite-dimensional. Werner, on the other hand, used rigid analytic uniformization for semistable abelian varieties. It does not seem possible to deduce the general case from her result by a simple application of the semistable reduction theorem, since Néron models are known to behave very badly under base change.

The method we take in this paper is first to formulate a local Tate duality type statement for the general case. This is a version for abelian varieties of the author's reformulation of a known duality theorem, where coefficients were finite flat group schemes (Suz13]). Our formulation crucially relies on the techniques of sheaves on the category of fields developed in Suz13. We prove that the duality we formulate here is equivalent to the conjunction of Grothendieck's conjecture and Šafarevič's conjecture, the latter of which was posed in Šaf61] and solved by Bégueri Bég81] (mixed characteristic case), Bester Bes78] (equal characteristic, good reduction) and Bertapelle Ber03] (equal characteristic, general reduction). Our duality is

\footnotetext{
${ }^{1}$ The conjecture may fail when $k$ is imperfect BB00. The results on the semistable case and the prime-to- $p$ part are valid for any (possibly imperfect or zero characteristic) residue field $k$. In this paper, we only consider the case that $k$ is perfect of positive characteristic.
} 
very functorial in an appropriate derived category that is strong enough to treat the infinite-dimensional groups involved, and well-suited with Galois descent. Therefore we are able to prove that if the duality is true over a finite Galois extension of $K$, then it is true over $K$. This and the semistable case cited above together imply Grothendieck's conjecture.

Here we sketch our formulation of the duality with coefficients in abelian varieties. Recall from Suz13 that a $k$-algebra is said to be rational if it is a finite direct product of the perfections (direct limit along Frobenii) of finitely generated fields over $k$, and ind-rational if it is a filtered union of rational $k$-subalgebras. We denote the category of ind-rational $k$-algebras by $k^{\text {indrat }}$. We can endow this category with the pro-étale topology ( $\overline{B S 15}$; see $\$ 2.1$ below for the details about the pro-étale topology in this ind-rational setting). We denote the resulting site by $\operatorname{Spec} k_{\text {proet }}^{\text {indrat }}$. The derived category of sheaves on Spec $k_{\text {proet }}^{\text {indrat }}$ is denoted by $D\left(k_{\text {proet }}^{\text {indrat }}\right)$ and the derived sheaf-Hom functor by $R \mathbf{H o m}_{k_{\text {proet }}^{\text {indrat }}}$. For an object $C \in D\left(k_{\text {proet }}^{\text {indrat }}\right)$, we define

$$
C^{\mathrm{SD}}=R \mathbf{H o m}_{k_{\text {proet }}^{\text {indrat }}}(C, \mathbb{Z})
$$

and call it the Serre dual of $C$ (cf. [Ser60, 8.4, Remarque], [Mil06, III, Thm. 0.14]). The double dual SDSD sends the perfection (inverse limit along Frobenii) of a prounipotent group to itself. It sends the perfection of a semi-abelian variety $G$ to its profinite Tate module placed in degree -1 . Hence the universal covering of $G$ placed in degree -1 gives a mapping cone of $G \rightarrow G^{\mathrm{SDSD}}$, which is uniquely divisible.

As in the beginning of the paper, let $K$ be a complete discrete valuation field with perfect residue field $k$ of characteristic $p>0$ and $A$ an abelian variety over $K$. We can regard the cohomology complex $R \Gamma(A)=R \Gamma(K, A)$ of $K$ as a complex of sheaves on Spec $k_{\text {proet }}^{\text {indrat }}$ in a suitable way. We denote the resulting complex by $R \boldsymbol{\Gamma}(A)$ and its $n$-th cohomology by $\mathbf{H}^{n}(A)$. The sheaf $\boldsymbol{\Gamma}(A)=\mathbf{H}^{0}(A)$ is represented by the perfection of the Greenberg transform of the Néron model of $A$. This is a proalgebraic group over $k$, and the reduction map induces an isomorphism $\pi_{0}(\boldsymbol{\Gamma}(A)) \stackrel{\sim}{\rightarrow} \pi_{0}\left(\mathcal{A}_{x}\right)$. The sheaf $\mathbf{H}^{1}(A)$ is ind-algebraic, and all higher cohomology vanishes. We have

$$
R \boldsymbol{\Gamma}(A)^{\mathrm{SD}}=R \mathbf{H o m}_{k_{\text {proet }}^{\text {indrat }}}(R \boldsymbol{\Gamma}(A), \mathbb{Q} / \mathbb{Z})[-1] .
$$

The trace morphism $R \boldsymbol{\Gamma}\left(\mathbf{G}_{m}\right) \rightarrow \mathbb{Z}$ given in [Suz13, Prop. 2.4.4] and the cup product formalism give a pairing

$$
R \boldsymbol{\Gamma}\left(A^{\vee}\right) \times R \boldsymbol{\Gamma}(A) \rightarrow \mathbb{Z}[1]
$$

in $D\left(k_{\text {proet }}^{\text {indrat }}\right)$. This induces morphisms

$$
R \boldsymbol{\Gamma}(A) \rightarrow R \boldsymbol{\Gamma}\left(A^{\vee}\right)^{\mathrm{SD}}[1] \quad \text { and } \quad R \boldsymbol{\Gamma}\left(A^{\vee}\right)^{\mathrm{SDSD}} \rightarrow R \boldsymbol{\Gamma}(A)^{\mathrm{SD}}[1] .
$$

We will prove the following.

Theorem A. The above defined morphism

$$
R \boldsymbol{\Gamma}\left(A^{\vee}\right)^{\mathrm{SDSD}} \rightarrow R \boldsymbol{\Gamma}(A)^{\mathrm{SD}}[1]
$$

in $D\left(k_{\text {proet }}^{\text {indrat }}\right)$ is an isomorphism.

We recall Šafarevič's conjecture, or a theorem of Bégueri and Bester-Bertapelle. In our terminology, Šafarevič's conjecture can be stated as the existence of a canonical isomorphism

$$
H^{1}\left(A^{\vee}\right) \cong \operatorname{Ext}_{k_{\text {proet }}^{\text {indrat }}}^{1}(\boldsymbol{\Gamma}(A), \mathbb{Q} / \mathbb{Z})
$$


for $k$ algebraically closed. The right-hand side is the Pontryagin dual the fundamental group $\pi_{1}(\boldsymbol{\Gamma}(A))$ of the proalgebraic group $\boldsymbol{\Gamma}(A)$ in the sense of Serre [Ser60]. Hence Safarevič's conjecture claims the existence of a perfect pairing

$$
H^{1}\left(A^{\vee}\right) \times \pi_{1}(\boldsymbol{\Gamma}(A)) \rightarrow \mathbb{Q} / \mathbb{Z}
$$

between the torsion group and the profinite group. Note that $\pi_{1}$ is indifferent to component groups and observe how this conjecture is complementary to Grothendieck's conjecture.

We will prove the following more precise form of the above theorem.

\section{Theorem B.}

(a) Theorem $\triangle$ is equivalent to the conjunction of Grothendieck's conjecture and Šafarevič's conjecture.

(b) If Theorem $A$ is true for $A \times_{K} L$ for a finite Galois extension $L$ of $K$

$$
R \boldsymbol{\Gamma}\left(L, A^{\vee}\right)^{\mathrm{SDSD}} \stackrel{\sim}{\rightarrow} R \boldsymbol{\Gamma}(L, A)^{\mathrm{SD}}[1],
$$

then it is true for the original $A$.

Since Šafarevič's conjecture is already a theorem, these results together with the semistable reduction theorem and Werner's result imply:

Theorem C. Grothendieck's conjecture is true.

In the next logically optional subsection, we explain the general picture of our formulation and proof. The organization of the paper will be explained in the subsection after next.

1.2. General picture. We explain the general picture of our proof of Grothendieck's conjecture without getting into the details or giving precise definitions, in order to motivate our very peculiar constructions. For the convenience of the reader, we repeat the main ideas in our previous paper Suz13. Notation is as in the previous subsection. Assume that $k$ is algebraically closed for simplicity.

Suppose for the moment that we have a nice complex $R \boldsymbol{\Gamma}(K, A)=R \boldsymbol{\Gamma}(A)$ of indor proalgebraic groups over $k$ for an abelian variety $A$ over $K$, so that its $k$-points is $R \Gamma(A)$ (hence $\mathbf{H}^{n}(A)=0$ for $n \geq 2$ by a cohomological dimension reason). The group $\boldsymbol{\Gamma}(A)$ is proalgebraic and $\mathbf{H}^{1}(A)$ ind-algebraic. What is nice about $R \boldsymbol{\Gamma}(A)$ is that it can capture the Néron component group $\pi_{0}\left(\mathcal{A}_{x}\right)$ and behaves well under base change.

To explain this, let $\mathfrak{p}_{K}$ be the maximal ideal of $\mathcal{O}_{K}$. We have a surjection

$$
\boldsymbol{\Gamma}(K, A)=\boldsymbol{\Gamma}\left(\mathcal{O}_{K}, \mathcal{A}\right)={\underset{\varliminf}{n}}_{\lim } \boldsymbol{\Gamma}\left(\mathcal{O}_{K} / \mathfrak{p}_{K}^{n}, \mathcal{A}\right) \stackrel{n=1}{\rightarrow} \mathcal{A}_{x}
$$

of proalgebraic groups with connected kernel, so $\pi_{0}(\boldsymbol{\Gamma}(A))=\pi_{0}\left(\mathcal{A}_{x}\right)$. This explains how $\pi_{0}\left(\mathcal{A}_{x}\right)$ can be functorially recovered from $\boldsymbol{\Gamma}(A)$. Here it is essential to put a proalgebraic group structure on $\boldsymbol{\Gamma}(A)$ to make sense of its $\pi_{0}$. On the other hand, the Hochschild-Serre spectral sequence

$$
R \Gamma(\operatorname{Gal}(L / K), R \boldsymbol{\Gamma}(L, A))=R \boldsymbol{\Gamma}(K, A)
$$

for a finite Galois extension $L / K$ shows that $R \boldsymbol{\Gamma}(K, A)$ can be recovered from $R \boldsymbol{\Gamma}(L, A)$ very cheaply, while there is no such simple relation for Néron models.

We expect some duality between the proalgebraic group $\boldsymbol{\Gamma}(A)$ and the indalgebraic group $\mathbf{H}^{1}\left(A^{\vee}\right)$ that takes care of $\pi_{0}(\boldsymbol{\Gamma}(A))$. This should be analogous 
to the usual local Tate duality in the finite residue field case. A little more precisely, let's expect that there should be an isomorphism

$$
R \boldsymbol{\Gamma}\left(A^{\vee}\right) \stackrel{\sim}{\rightarrow} R \operatorname{Hom}_{k}(R \boldsymbol{\Gamma}(A), \mathbb{Q} / \mathbb{Z})
$$

up to some completion of the left-hand side. Here $R \mathbf{H o m}_{k}$ is some internal $R$ Hom functor for the category of ind- or proalgebraic groups over $k$. The actual duality statement needs the completion or double-dual as in previous subsection, since semiabelian varieties (appearing in $\mathcal{A}_{x}$ ) are not double-dual invariant (while unipotent groups are so). Let's ignore the double-dual. This duality statement is robust for Galois descent: if it is true for $A$ over a finite Galois extension $L / K$, then it is true for $A$ over $K$, basically by the Hochschild-Serre spectral sequence above.

The statement is equivalent to the conjunction of Grothendieck's and Šafarevič's conjectures as follows. The isomorphism (ignoring the completion) gives a hyperext spectral sequence

$$
E_{2}^{i j}=\mathbf{E x t}_{k}^{i}\left(\mathbf{H}^{-j}(A), \mathbb{Q} / \mathbb{Z}\right) \Longrightarrow \mathbf{H}^{i+j}\left(A^{\vee}\right) .
$$

The functor $\mathbf{E x t}_{k}^{i}(\cdot, \mathbb{Q} / \mathbb{Z})$ is dual to the $i$-th homotopy group functor by $\operatorname{Ser60}, \S 5$, Cor. to Prop. 7], hence zero for $i \geq 2$ by [Ser60, §10, Thm. 2]. Therefore this spectral sequence degenerates at $E_{2}$ and becomes (ignoring the term $\mathbf{H o m}_{k}\left(\mathbf{H}^{1}(A), \mathbb{Q} / \mathbb{Z}\right)$ ) an exact sequence and an isomorphism

$$
\begin{aligned}
0 \rightarrow \operatorname{Ext}_{k}^{1}\left(\mathbf{H}^{1}(A), \mathbb{Q} / \mathbb{Z}\right) & \rightarrow \boldsymbol{\Gamma}\left(A^{\vee}\right) \rightarrow \operatorname{Hom}_{k}(\boldsymbol{\Gamma}(A), \mathbb{Q} / \mathbb{Z}) \rightarrow 0, \\
\mathbf{H}^{1}\left(A^{\vee}\right) & \cong \mathbf{E x t}_{k}^{1}(\boldsymbol{\Gamma}(A), \mathbb{Q} / \mathbb{Z})
\end{aligned}
$$

(with a completion to $\boldsymbol{\Gamma}\left(A^{\vee}\right)$ ). The isomorphism gives Šafarevič's conjecture. As [Mil06, III Lem. 0.13 (c)] suggests, the group $\operatorname{Ext}_{k}^{1}\left(\mathbf{H}^{1}(A), \mathbb{Q} / \mathbb{Z}\right)$ should be connected. Hence the exact sequence should be a connected-étale sequence. The connected part gives the statement that the identity component of $\boldsymbol{\Gamma}\left(A^{\vee}\right)$ up to completion is $\operatorname{Ext}_{k}^{1}\left(\mathbf{H}^{1}(A), \mathbb{Q} / \mathbb{Z}\right)$. This is dual to Šafarevič's conjecture. The étale part gives an isomorphism

$$
\pi_{0}\left(\boldsymbol{\Gamma}\left(A^{\vee}\right)\right) \cong \operatorname{Hom}_{k}\left(\pi_{0}(\boldsymbol{\Gamma}(A)), \mathbb{Q} / \mathbb{Z}\right) .
$$

Writing the groups as Néron component groups, we get the isomorphism predicted by Grothendieck's conjecture.

Here it is very important to have a complex, $R \boldsymbol{\Gamma}(A)$, of ind- or proalgebraic groups, and work in a derived category. Looking at the terms individually seriously breaks the good behavior under base change.

How can we define such a complex? A naive approach is the following. For a perfect $k$-algebra $R$, we define its canonical lift " $R \otimes_{k} K$ " to $K$ to be

$$
\mathbf{K}(R)=\left(W(R) \hat{\otimes}_{W(k)} \mathcal{O}_{K}\right) \otimes_{\mathcal{O}_{K}} K,
$$

where $W$ is the affine scheme of Witt vectors of infinite length. More explicitly, if $K=W(k)[1 / p][x] /(f(x))$ with some Eisenstein polynomial $f(x) \in W(k)[x]$, then $\mathbf{K}(R)=W(R)[1 / p][x] /(f(x))$, and if $K=k((T))$, then $\mathbf{K}(R)=R[[T]][1 / T]$. If $R=k^{\prime}$ is a perfect field, the ring $\mathbf{K}\left(k^{\prime}\right)$ is a complete discrete valuation field obtained from $K$ by extending the residue field from $k$ to $k^{\prime}$. Take an injective resolution $I$ of $A$ over the fppf site of $K$. Consider the complex of presheaves

$$
R \mapsto \Gamma\left(\mathbf{K}(R), I^{\cdot}\right)
$$


on the category of perfect $k$-algebras. Its (pro-)étale sheafification is the candidate of our complex. Its $n$-th cohomology is the sheafification of the presheaf

$$
R \mapsto H^{n}(\mathbf{K}(R), A) .
$$

But this fppf cohomology group is very difficult to calculate. It is a classical object only if we restrict the $R$ 's to be perfect fields. This means that we can only obtain the generic behavior of the sheaf from our classical knowledge.

But generic behavior is sufficient to describe algebraic groups in view of Weil's theory of birational groups. This means that there is no information lost by treating proalgebraic groups as functors on the category of ind-rational $k$-algebras, which is essentially the category of perfect fields over $k$. Now the sheafification of the complex of presheaves

$$
k^{\prime} \mapsto \Gamma\left(\mathbf{K}\left(k^{\prime}\right), I^{\cdot}\right)
$$

on our ind-rational pro-étale site Spec $k_{\text {proet }}^{\text {indrat }}$ yields the sought-for object $R \boldsymbol{\Gamma}(A)$. This explain why this strange site Spec $k_{\text {proet }}^{\text {indrat }}$ is needed in this paper. Moreover, this type of complexes and cohomology groups looks very similar to the well-known general description of higher pushforward sheaves. Therefore we are tempted to define a morphism of sites

$$
\pi: \operatorname{Spec} K_{\mathrm{fppf}} \rightarrow \operatorname{Spec} k_{\text {proet }}^{\text {indrat }}
$$

corresponding to the functor $k^{\prime} \mapsto \mathbf{K}\left(k^{\prime}\right)$ on the underlying categories, so that we have a more systematic definition $R \boldsymbol{\Gamma}(A)=R \pi_{*} A$. In fact, a little care and modifications are needed for continuity and exactness of pullback.

Now the picture is the following. The duality we want should be a relative duality for the morphism $\pi$. The cup product formalism in site theory will give us the duality morphism (1.2.1). The derived sheaf-Hom functor $R \mathbf{H o m}_{k_{\text {proet }}^{\text {indrat }}}$ on the site Spec $k_{\text {proet }}^{\text {indrat }}$ should give the internal $R$ Hom functor on the category of ind-proalgebraic groups.

All we do in this paper is to carry out these ideas rigorously making necessary corrections to imprecise ideas. There are three difficulties to overcome. One is to show that $R \mathbf{H o m}_{k_{\text {proet }}^{\text {indrat }}}(G, H)$ behaves well for ind-proalgebraic groups (i.e., indobjects of proalgebraic groups) $G, H$. We already know this for proalgebraic $G$ and algebraic $H$ by Suz13, Thm. 2.1.5]. To extend this result for ind-proalgebraic groups, we will need heavy derived limit arguments following Kashiwara-Schapira [KS06. Chap. 15]. Second, cohomology groups of the form $H^{n}\left(\mathbf{K}\left(k^{\prime}\right), A\right)$ are not completely classical when $k^{\prime}$ has infinitely many direct factors and Spec $k^{\prime}$ as a topological space is profinite. The ring $\mathbf{K}\left(k^{\prime}\right)$ is a finite direct product of complete discrete valuation fields if $k^{\prime}$ has only finitely many direct factors, but otherwise it is quite complicated. We study some site-theoretic properties of the $\operatorname{ring} \mathbf{K}\left(k^{\prime}\right)$ by approximation by complete discrete valuation subfields. In [Suz13, §2.5], we calculated $H^{n}\left(\mathbf{K}\left(k^{\prime}\right), \mathbf{G}_{m}\right)$. We need to further develop the techniques used there in order to calculate $H^{n}\left(\mathbf{K}\left(k^{\prime}\right), A\right)$ for an abelian variety $A$. The third point is that we need to redo Bester's work Bes78 within the style of this paper. This is lengthy, but there is essentially no new idea needed in this part.

1.3. Organization. This paper is organized as follows. In $\oint_{2}$ we study how to treat ind-proalgebraic groups over $k$ as sheaves on $\operatorname{Spec} k_{\text {proet }}^{\text {indrat }}$. In 33 , we view cohomology of $K$ with various coefficients as sheaves on Spec $k_{\text {proet }}^{\text {indrat }}$ and compute 
it. In 4 , we construct the duality morphism of Theorem $\mathrm{A}$ and show that it induces two pairings

$$
\pi_{0}\left(\mathcal{A}_{x}^{\vee}\right) \times \pi_{0}\left(\mathcal{A}_{x}\right) \rightarrow \mathbb{Q} / \mathbb{Z}, \quad H^{1}\left(A^{\vee}\right) \times \pi_{1}(\boldsymbol{\Gamma}(A)) \rightarrow \mathbb{Q} / \mathbb{Z}
$$

The perfectness of these pairings is equivalent to Theorem $\mathrm{A}$. In $₫ 5$, we show that the first pairing agrees with Grothendieck's pairing and the second with Bégueri-BesterBertapelle's pairing. This proves TheoremB(国) and the semistable case of Theorem A. In 96 , we prove that Galois descent works for Theorem A, namely Theorem B (b). In 97 , we summarize the results of the preceding sections to conclude the proof of Theorems $\mathrm{A}, \mathrm{B}$ and $\mathrm{C}$, which finishes the proof of Grothendieck's conjecture.

In 88 , we formulate and prove an analogue of Theorem $\mathrm{A}$ for tori. Most of the results of this section has already been obtained by Bégueri (loc.cit.), Xarles Xar93 and Bertapelle-González-Avilés [BGA15. In 99, the dualities for abelian varieties and tori are combined into a duality for 1-motives. This extends the finite residue field case of the duality shown by Harari-Szamuely [HS05. In 10, we show, in the finite residue field case, how to pass from the above sheaf setting to the classical setting. In Appendix A, we explain how to treat proalgebraic groups that are proschemes but not schemes. We do this by extending the pro-fppf topology introduced in [Suz13] for affine schemes to proalgebraic proschemes.

Acknowledgement. The author expresses his deep gratitude to Kazuya Kato for his support and helpful discussions. This paper is seriously based on his ideas on local duality [Kat86, Introduction], Kat91, §3.3]. The author is grateful to Clifton Cunningham and David Roe, who explained the author relations between their work CR15] and Serre's local class field theory Ser61. Their invitation, hospitality and useful discussions on tori over local fields at Calgary were valuable. The author would like to thank Alessandra Bertapelle and Cristian D. GonzálezAvilés, who informed the author of their work BGA15 and encouraged to give details on the duality with coefficients in tori. Bertapelle also helped the author to understand several points in her work on Šafarevič's conjecture. Special thanks to Cédric Pépin for sharing his interest in Grothendieck's conjecture and useful discussions, to Michal Bester for acknowledging our treatment of his work in this paper, and to the referee for their very careful reading and suggestions to make the paper more readable.

Notation. We fix a perfect field $k$ of characteristic $p>0$. A perfect field over $k$ is said to be finitely generated if it is the perfection (direct limit along Frobenii) of a finitely generated field over $k$. The same convention is applied to morphisms of perfect $k$ algebras or $k$-schemes being finite type, finite presentation, etc. The categories of sets and abelian groups are denoted by Set and Ab, respectively. Set theoretic issues are omitted for simplicity as the main results hold independent of the choice of universes. The opposite category of a category $\mathcal{C}$ is denoted by $\mathcal{C}^{\text {op }}$. The procategory and indcategory of $\mathcal{C}$ are denoted by $\mathrm{PC}$ and $\mathrm{IC}$, respectively, so that IPC $=\mathrm{I}(\mathrm{PC})$ is the ind-procategory. All group schemes (except for Galois groups) are assumed to be commutative. We say that a (commutative) étale group scheme over $k$ is finitely generated if its group of geometric points is finitely generated as an abelian group. A lattice is a finitely generated étale group with no torsion or, equivalently, a finite free abelian group with a Galois action. For an abelian category $\mathcal{C}$, we denote by $D^{b}(\mathcal{C})$, $D^{+}(\mathcal{C}), D^{-}(\mathcal{C}), D(\mathcal{C})$ its bounded, bounded below, bounded above and unbounded derived categories, respectively. If we say $A \rightarrow B \rightarrow C$ is a distinguished triangle 
in a triangulated category, we implicitly assume that a morphism $C \rightarrow A[1]$ to the shift of $A$ is given and $A \rightarrow B \rightarrow C \rightarrow A[1]$ is distinguished. For a Grothendieck site $S$ and a category $\mathcal{C}$, we denote by $\mathcal{C}(S)$ the category of sheaves on $S$ with values in $\mathcal{C}$. For an object $X$ of $S$, the category $S / X$ of objects of $S$ over $X$ is equipped with the induced topology (AGV72, III, §3]), which is the localization of $S$ at $X$ (AGV72, III, §5]). The category $D^{*}(\operatorname{Ab}(S))$ for $*=b,+,-$ or (blank) is also denoted by $D^{*}(S)$. By a continuous map $f: S^{\prime} \rightarrow S$ between sites $S^{\prime}$ and $S$, we mean a continuous functor from the underlying category of $S$ to that of $S^{\prime}$, i.e., the right composition (or the pushforward $f_{*}$ ) sends sheaves on $S^{\prime}$ to sheaves on $S$. By a morphism $f: S^{\prime} \rightarrow S$ of sites we mean a continuous map whose pullback functor $\operatorname{Set}(S) \rightarrow \operatorname{Set}\left(S^{\prime}\right)$ is exact. For an abelian category $\mathcal{C}$, we denote by $\operatorname{Ext}_{\mathcal{C}}^{i}$ the $i$-th Ext functor for $\mathcal{C}$. If $\mathcal{C}=\mathrm{Ab}(S)$, we also write $\operatorname{Ext}_{S}^{i}=\operatorname{Ext}_{\mathcal{C}}^{i}$. The sheaf-Hom and sheaf-Ext functors are denoted by $\mathbf{H o m}_{S}$ and $\mathbf{E x t}_{S}^{n}$, respectively. For sites such as $\operatorname{Spec} k_{\text {proet }}^{\text {indrat }}$, we also use $\operatorname{Ext}_{k_{\text {proet }}^{\text {indrat }}}, \operatorname{Ab}\left(k_{\text {proet }}^{\text {indrat }}\right)$ etc. omitting Spec from the notation. Similarly, cohomology of the site Spec $k_{\text {proet }}^{\text {indrat }}$ for example is denoted by $R \Gamma\left(k_{\text {proet }}^{\text {indrat }}, A\right)$, where $A$ is a sheaf of abelian groups.

Statements (theorems, propositions etc.), remarks and equations share the numbering system. Their numbers are preceded by (sub)(sub)section numbers and surrounded by parentheses. Therefore (6.2) for example means the second one of the statements, remarks and equations in $\S 6$, and (5.2.2.3) means the third one of such in $§ 5.2 .2$. Listed items are labeled by letters. Therefore (b) means the second item in a list. As a consequence, (2.2.1) (c) means the third item in the list in $(2.2 .1)$.

\section{Site-TheORETIC PRELIMINARIES}

Let $k$ be a perfect field of characteristic $p>0$. In this section, we introduce the ind-rational pro-étale site $\operatorname{Spec} k_{\text {proet }}^{\text {indrat }}$. We also recall the perfect pro-fppf site Spec $k_{\text {profppf }}^{\text {perf }}$ from [Suz13. We call a help from Spec $k_{\text {profppf }}^{\text {perf }}$ to establish a basic method of treating ind-proalgebraic groups as sheaves on Spec $k_{\text {proet }}^{\text {indrat }}$. This needs to extend the result [Suz13, Thm. 2.1.5] on Ext groups of proalgebraic groups to ind-proalgebraic groups. For this, we develop a general study on the derived Hom functor $R$ Hom on ind-procategories. Then we can embed the derived category of ind-proalgebraic groups into the derived category of sheaves on Spec $k_{\text {proet }}^{\text {indrat }}$. We also extend Serre duality (Ser60, 8.4, Remarque], [Mil06, III, Thm. 0.14]) on perfect unipotent groups (= quasi-algebraic unipotent groups) to proalgebraic and indalgebraic groups.

At the end of this section, we introduce the notion of P-acyclicity. In the next section, we will view the cohomology of complete discrete valuation fields with residue field $k$ as sheaves on the ind-rational étale site Spec $k_{\mathrm{et}}^{\text {indrat }}$ first and then as sheaves on Spec $k_{\text {proet }}^{\text {indrat }}$ by sheafification. The cohomology of the pro-étale sheafification of an étale sheaf is difficult to calculate in general. In the situations we are interested in, however, we will see that most of these étale sheaves are P-acyclic. This means that the associated pro-étale sheaves still remember the original étale sheaves and hence the cohomology of the original complete discrete valuation field. This notion is also useful when we want to obtain some information specific to a non-closed residue field $k$. See (7.3) and (8.9). 
2.1. Sites and algebraic groups: setup and first properties. As in [Suz13, Def. 2.1.1], we say that a perfect $k$-algebra $k^{\prime}$ is rational if it is a finite direct product of finitely generated perfect fields over $k$, and ind-rational if it is a filtered union of rational $k$-subalgebras. Since any perfect field over $k$ is ind-rational, we know that a $k$-algebra is ind-rational if and only if it can be written as a filtered union of finite products of (not necessarily finitely generated) perfect fields over $k$. The rational (resp. ind-rational) $k$-algebras form a full subcategory of the category of perfect $k$ algebras, which we denote by $k^{\text {rat }}$ (resp. $\left.k^{\text {indrat }}\right)$. Since a $k$-algebra homomorphism from $k^{\prime} \in k^{\text {rat }}$ to $k^{\prime \prime}=\bigcup k_{\lambda}^{\prime \prime} \in k^{\text {indrat }}$ factors through some $k_{\lambda}^{\prime \prime} \in k^{\text {rat }}$, we know that $k^{\text {indrat }}$ is equivalent to the indcategory of $k^{\text {rat } 2}$ We define the rational (resp. ind-rational) étale site of $k$ to be the étale site on $k^{\text {rat }}$ (resp. $k^{\text {indrat}}$ ) (Suz13, Def. 2.1.3]). These sites are denoted by Spec $k_{\mathrm{et}}^{\text {rat }}$ and $\operatorname{Spec} k_{\mathrm{et}}^{\text {indrat }}$, respectively.

We also introduce the pro-étale topology on $k^{\text {indrat }}$. The pro-étale topology for schemes is introduced in BS15. We throughout use the affine variant of the proétale site [BS15, Def. 4.2.1, Rmk. 4.2.5], which behaves simpler for limit arguments. Note that any $k$-algebra étale over an ind-rational $k$-algebra is ind-rational Suz13, Prop. 2.1.2]. Hence if $k^{\prime} \in k^{\text {indrat }}$ and $R$ is a perfect $k^{\prime}$-algebra, then $R$ is ind-étale ([BS15, Def. 2.2.1.5]) over $k^{\prime}$ if and only if $R \cong{\underline{\lim _{\lambda}}}_{\lambda} k_{\lambda}^{\prime}$ for some filtered direct system $\left\{k_{\lambda}^{\prime}\right\}$ of étale $k^{\prime}$-algebras ind-rational over $k$. In particular, such an $R$ itself is ind-rational over $k$. Therefore we can introduce the pro-étale topology on the category $k^{\text {indrat }}$. That is, a covering of an ind-rational $k$-algebra $k^{\prime}$ is a finite family $\left\{k_{i}^{\prime}\right\}$ of ind-étale $k^{\prime}$-algebras such that $\prod k_{i}^{\prime}$ is faithfully flat over $k^{\prime}$. We call the resulting site the ind-rational pro-étale site and denote it by $\operatorname{Spec} k_{\text {proet }}^{\text {indrat }}$.

Some care is needed for localizations (see Notation) of Spec $k_{\mathrm{et}}^{\mathrm{rat}}$, Spec $k_{\mathrm{et}}^{\text {indrat }}$ and Spec $k_{\text {proet }}^{\text {indrat }}$, which comes from subtleties of the underlying categories $k^{\text {rat }}$ and $k^{\text {indrat }}$. See [Suz13, the paragraphs after Def. 2.1.3] for the details. We quickly recall the facts and notation there. As in Notation in this paper, for $k^{\prime} \in k^{\text {indrat }}$, the category of objects over $k^{\prime}$ in $k^{\text {indrat }}$ (i.e. the category of $k^{\prime}$-algebras ind-rational over $k$ ) is denoted by $k^{\text {indrat }} / k^{\prime}$, and the localization of Spec $k_{\text {proet }}^{\text {indrat }}$ at $k^{\prime}$ is denoted by Spec $k_{\text {proet }}^{\text {indrat }} / k^{\prime}$. If $k^{\prime}$ is a field that is in $k^{\text {rat }}$ or algebraic over $k$, then $k^{\text {indrat }}=$ $k^{\text {indrat }} / k^{\prime}$ Suz13, loc. cit.], hence

$$
\operatorname{Spec} k_{\mathrm{et}}^{\text {indrat }}=\operatorname{Spec} k_{\mathrm{et}}^{\text {indrat }} / k^{\prime}, \quad \operatorname{Spec} k_{\mathrm{proet}}^{\text {indrat }}=\operatorname{Spec} k_{\mathrm{proet}}^{\text {indrat }} / k^{\prime} .
$$

For any $k^{\prime} \in k^{\text {rat }}$, we define

$$
\begin{gathered}
k^{\text {indrat }}:=k^{\text {indrat }} / k^{\prime}, \\
\operatorname{Spec} k_{\text {et }}^{\text {indrat }}:=\operatorname{Spec} k_{\text {et }}^{\text {indrat }} / k^{\prime}, \quad \operatorname{Spec} k_{\text {proet }}^{\text {indrat }}:=\operatorname{Spec} k_{\text {proet }}^{\text {indrat }} / k^{\prime} .
\end{gathered}
$$

Let $k^{\text {perf }}$ be the category of perfect $k$-algebras. Some general results on perfect schemes in modern language can be found in [BS17, §3] and [BGA17. On $k^{\text {perf }}$, we can introduce the étale, (affine) pro-étale [BS15, Def. 4.2.1, Rmk. 4.2.5] and pro-fppf [Suz13, Def. 3.1.3] topologies. We denote the resulting sites by Spec $k_{\text {et }}^{\text {perf }}$,

\footnotetext{
${ }^{2}$ Do not confuse $k^{\text {indrat }}$ with another indcategory $\mathrm{IC}$, where $\mathcal{C}$ is the category of finite products of not necessarily finitely generated perfect fields over $k$ with $k$-algebra homomorphisms. There are natural functors $k^{\text {indrat }} \rightarrow \mathrm{IC} \rightarrow k^{\text {indrat }}$. The first one is fully faithful and the second one is essentially surjective, with composite the identity functor. The object of $\mathcal{C}$ given by the perfection of the field $k\left(x_{1}, x_{2}, \ldots\right)$ and the ind-object in $\mathcal{C}$ consisting of the increasing family of the perfections of the fields $k\left(x_{1}, \ldots, x_{n}\right)$ are not isomorphic in $\mathrm{IC}$, but become isomorphic in $k^{\text {indrat }}$.
} 
Spec $k_{\text {proet }}^{\text {perf }}$, Spec $k_{\text {profppf }}^{\text {perf }}$, respectively. A covering in Spec $k_{\text {proet }}^{\text {perf }}$ is a finite family $\left\{R \rightarrow S_{i}\right\}$ with each $S_{i}$ ind-étale over $R$ and $\prod S_{i}$ faithfully flat over $R$. For Spec $k_{\text {profppf }}^{\text {perf }}$, recall from [Suz13, §3.1] that a homomorphism $R \rightarrow S$ in $k^{\text {perf }}$ is said to be flat of finite presentation if $S$ is the perfection of a flat $R$-algebra of finite presentation. Also, a homomorphism $R \rightarrow S$ in $k^{\text {perf }}$ is said to be flat of ind-finite presentation if it can be written as a filtered direct limit of flat homomorphisms $R \rightarrow S_{\lambda}$ of finite presentation ([Suz13, Def. 3.1.1]). A covering in Spec $k_{\text {profppf }}^{\text {perf }}$ is a finite family $\left\{R \rightarrow S_{i}\right\}$ with each $S_{i}$ flat of ind-finite presentation and $\prod S_{i}$ faithfully flat over $R$. For a perfect $k$-algebra $R$, the category of objects over $R$ in $k^{\text {perf }}$ is nothing but the category of perfect $R$-algebras, in contrast to the case of the category of ind-rational $k$-algebras $k^{\text {indrat }}$. Hence we will write the localization of Spec $k_{\text {profppf }}^{\text {perf }} R$ by Spec $k_{\text {profppf }}^{\text {perf }} / R=\operatorname{Spec} R_{\text {profppf }}^{\text {perf }}$. Similar notation applies to Spec $k_{\text {et }}^{\text {perf }}$ and Spec $k_{\text {proet }}^{\text {perf }}$.

For $R \in k^{\text {perf }}$, we define the small pro-étale site $\operatorname{Spec} R_{\text {proet }}$ of $R$ to be the category $R_{\text {proet }}$ of ind-étale $R$-algebras ([BS15, Def. 2.2.1.5]) with the topology induced from Spec $k_{\text {proet }}^{\text {perf }}$. That is, a covering $\left\{R_{i}^{\prime}\right\}$ of an object $R^{\prime} \in R_{\text {proet }}$ is a finite family of ind-étale $R^{\prime}$-algebras such that $\prod R_{i}^{\prime}$ is faithfully flat over $R^{\prime}$.

We have the following commutative diagram of continuous maps of sites:

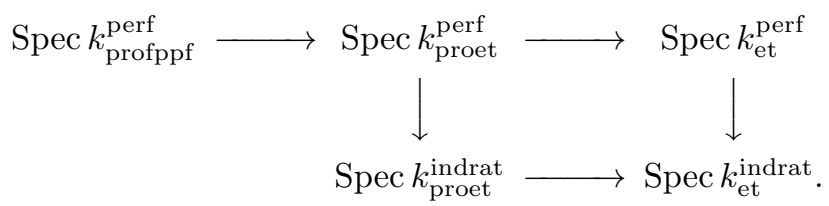

All the maps are defined by the identity. The horizontal maps are morphisms of sites (i.e., have exact pullback functors), but the vertical ones are not Suz13, Prop. 3.2.3].

Let Alg/ $k$ be the category of quasi-algebraic groups (commutative, as assumed throughout the paper) over $k$ in the sense of Serre Ser60. Recall that a quasialgebraic group is the perfection (inverse limit along Frobenii) of an algebraic group Ser60, $\S 1.2$ Déf. 2; §1.4, Prop. 10], [Pép14, Prop. 1.2.10]. The category Alg/ $k$ is an abelian category [Ser60, §1.2, Prop. 5]. We simply call an object of the procategory PAlg $/ k$ a proalgebraic group following [Ser60, §2.1, Déf. 1; §2.6, Prop. 12]. Similarly, we call an object of the indcategory IAlg/ $k$ an ind-algebraic group and an object of the ind-procategory IPAlg $/ k=\mathrm{I}(\mathrm{PAlg} / k)$ an ind-proalgebraic group. (Therefore we will not say $\mathbb{Z}$ ind-algebraic or ind-proalgebraic in this paper.) Note that the indcategory (and hence also the procategory) of an abelian category is an abelian category [KS06, Thm. 8.6.5 (i)]. Following [Ser60, §1.3], we say that a quasi-algebraic group is a unipotent group, a torus or an abelian variety if it is the perfection of a unipotent group, a torus or an abelian variety, respectively. Let $\mathrm{Alg}_{\mathrm{uc}} / k \subset \mathrm{Alg} / k$ be the full subcategory consisting of those whose identity component is unipotent. This is the direct product of the category of (not necessarily connected) unipotent quasi-algebraic groups and the category of finite étale groups of order prime to $p$. We have fully faithful exact embeddings of abelian categories

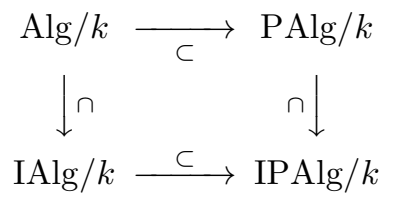


by KS06 Thm. 8.6.5 (ii)]. Let LAlg/ $k$ be the category of perfections of smooth group schemes over $k$ (which contains $\mathbb{Z}$ but is not abelian). Let Et/ $k$ be the category of étale group schemes over $k$. Let FGEt/ $k$ be its full subcategory consisting of finitely generated étale groups, namely those whose group of geometric points is a finitely generated abelian group. Let FEt/ $k$ be the full subcategory consisting of finite étale group schemes. The categories FEt $/ k, \mathrm{FGEt} / k$ and Et $/ k$ are abelian. We have fully faithful embeddings FEt $/ k \subset \mathrm{FGEt} / k \subset \mathrm{Et} / k \subset \mathrm{LAlg} / k$. The identity component of $A \in \mathrm{LAlg} / k$ is denoted by $A_{0}{ }^{3}$ The formation of $A_{0}$ is functorial, so the notion and notation of identity component extend to any $A \in \mathrm{IAlg} / k, \mathrm{PAlg} / k$ or IPAlg $/ k$. We define $\pi_{0}(A)=A / A_{0}$, which is in IFEt $/ k, \mathrm{PFEt} / k$ or IPFEt $/ k$ in each case. We say that $A \in \mathrm{IPAlg} / k$ is connected if $\pi_{0}(A)=0$. We have natural additive functors IPAlg $/ k, \mathrm{LAlg} / k \rightarrow \mathrm{Ab}\left(k_{\text {proet }}^{\text {indrat }}\right)$.

We say that a sheaf $A$ of abelian groups on a site $S$ is acyclic if $H^{n}(X, A)=0$ for any object $X$ of $S$ and any $n \geq 1$. If $S, S^{\prime}$ are sites defined by pretopologies, and if $u$ is a functor from the underlying category of $S$ to that of $S^{\prime}$ that sends coverings to coverings and $u\left(Y \times_{X} Z\right)=u(Y) \times_{u(X)} u(Z)$ whenever $Y \rightarrow X$ appears in a covering family, then $u$ defines a continuous map $f: S^{\prime} \rightarrow S$, and $f_{*}$ sends acyclic sheaves to acyclic sheaves and hence induces the Leray spectral sequence $R \Gamma\left(X, R f_{*} A^{\prime}\right)=R \Gamma\left(u(X), A^{\prime}\right)$ for any $X \in S$ and $A^{\prime} \in \mathrm{Ab}\left(S^{\prime}\right)$ [Art62, §II.4].

\section{Proposition (2.1.2).}

(a) On any of the sites in the diagram (2.1.1), cohomology of any object of the site commutes with filtered direct limits of coefficient sheaves. Products and filtered direct limits of acyclic sheaves are again acyclic.

(b) The sites $\operatorname{Spec} k_{\text {profppf }}^{\text {perf }}$, Spec $k_{\text {proet }}^{\text {perf }}$ and $\operatorname{Spec} k_{\text {et }}^{\text {perf }}$ are coherent, i.e., the objects of their underlying categories are quasi-compact and stable under finite inverse limits.

(c) Let $f:$ Spec $k_{\text {proet }}^{\text {perf }} \rightarrow$ Spec $k_{\text {proet }}^{\text {indrat }}$ be the continuous map defined by the identity. Then $f$ induces isomorphisms on cohomology. More precisely, $f_{*}$ is exact, $f_{*} f^{*}=$ id on $\mathrm{Ab}\left(k_{\text {proet }}^{\text {indrat }}\right)$ (hence $f^{*}$ is a fully faithful embedding), and

$$
R \Gamma\left(k_{\text {proet }}^{\text {indrat }} / k^{\prime}, f_{*} A\right)=R \Gamma\left(k_{\text {proet }}^{\text {perf }}, A\right)
$$

for any $k^{\prime} \in k^{\text {indrat }}$ and $A \in \mathrm{Ab}\left(k_{\text {proet }}^{\text {perf }}\right)$, where the left-hand side is the cohomology of the site Spec $k_{\text {proet }}^{\text {indrat }}$ at $k^{\prime}$. The same is true for the étale version $\operatorname{Spec} k_{\text {et }}^{\text {perf }} \rightarrow \operatorname{Spec} k_{\text {et }}^{\text {indrat }}$.

(d) In the categories $\operatorname{Set}\left(k_{\text {profppf }}^{\text {perf }}\right)$, $\operatorname{Set}\left(k_{\text {proet }}^{\text {perf }}\right)$ and $\operatorname{Set}\left(k_{\text {proet }}^{\text {indrat }}\right)$, the product of any family of surjections is a surjection. This implies that $\mathrm{Ab}\left(k_{\text {profppf }}^{\text {perf }}\right)$, $\mathrm{Ab}\left(k_{\text {proet }}^{\text {perf }}\right)$ and $\mathrm{Ab}\left(k_{\text {proet }}^{\text {indrat }}\right)$ are $A B_{4}^{*}$ categories.

(e) The natural functor from $\mathrm{PAlg} / k$ to any of $\mathrm{Ab}\left(k_{\text {profppf }}^{\text {perf }}\right), \mathrm{Ab}\left(k_{\text {proet }}^{\text {perf }}\right), \mathrm{Ab}\left(k_{\text {proet }}^{\text {indrat }}\right)$ is exact.

\footnotetext{
${ }^{3}$ More customary notation is $A^{0}$ or $A^{\circ}$. We prefer the subscript 0 in this paper, however, since we extensively use derived categories and $A^{0}$ may be ambiguous to the zeroth term of a complex $\cdots \rightarrow A^{0} \rightarrow A^{1} \rightarrow \cdots$. A circle $\circ$ in the script size looks too similar to a zero 0 and $A^{\circ}$ may still be confusing in this paper. Moreover, we use below tons of duality operations such as the Serre dual $A^{\mathrm{SD}}$, the Pontryagin dual $A^{\mathrm{PD}}$, the dual abelian variety $A^{\vee}$ etc. We want to distinguish such contravariant operations from the covariant operation $A \mapsto A_{0}$.
} 
(f) Let $A=\lim _{\lambda} A_{\lambda} \in \mathrm{PAlg} / k$ with $A_{\lambda} \in \mathrm{Alg} / k$. Let $R \lim _{\lambda}$ be the derived functor of $\lim _{\lambda}$ considered in either $D\left(k_{\text {profpp }}^{\text {perf }}\right), D\left(k_{\text {proet }}^{\text {perf }}\right)$ or $D\left(k_{\text {proet }}^{\text {indrat }}\right)$. Then we have $R \varliminf_{\lambda} A_{\lambda}=A$ in each case.

(g) Let $f:$ Spec $k_{\text {profppf }}^{\text {perf }} \rightarrow$ Spec $k_{\text {proet }}^{\text {perf }}$ be the morphism defined by the identity. Let $g$ be either the morphism Spec $k_{\text {proet }}^{\text {perf }} \rightarrow$ Spec $k_{\text {et }}^{\text {perf }}$ or Spec $k_{\text {proet }}^{\text {indrat }} \rightarrow$ Spec $k_{\mathrm{et}}^{\text {indrat }}$ defined by the identity. If $A \in \operatorname{IPAlg} / k$, the pro-fppf cohomology with coefficients in $A$ agrees with the pro-étale cohomology: $R f_{*} A=A$. If $A \in \mathrm{IAlg} / k$ or $\mathrm{LAlg} / k$, the pro-fppf cohomology with coefficients in $A$ agrees with the étale cohomology: $R f_{*} A=A$ and $R g_{*} A=A$.

Proof. (回) This is true for any site defined by finite coverings. See Mil80, III, Rmk. 3.6] for the statement that cohomology commutes with filtered direct limits. It follows that filtered direct limits of acyclics are acyclic. If a family of sheaves has vanishing higher Čech cohomology, then so is the product. We can deduce the corresponding statement for the derived functor cohomology by Mil80, III, Prop. $2.12]$.

(b) Obvious.

(IC) The exactness of $f_{*}$ is obvious. We know that $f_{*}$ commutes with sheafification and $f_{*} f^{-1}=\mathrm{id}$, where $f^{-1}$ is the pullback for presheaves. Hence $f_{*} f^{*}=\mathrm{id}$. The stated equality is the Leray spectral sequence for $f$, which is available by the remark in the paragraph before the proposition. The same proof works for the étale version Spec $k_{\text {et }}^{\text {perf }} \rightarrow$ Spec $k_{\text {et }}^{\text {indrat }}$.

(d) Let $\left\{G_{\lambda} \rightarrow F_{\lambda}\right\}$ be a family of surjections in $\operatorname{Set}\left(k_{\text {profppf }}^{\text {perf }}\right)$. Let $R \in k^{\text {perf }}$ and $\left\{s_{\lambda}\right\} \in \prod F_{\lambda}(R)$. For each $\lambda$, there are an object $S_{\lambda} \in k^{\text {perf }}$ faithfully flat of ind-finite presentation over $R$ and a section $t_{\lambda} \in G_{\lambda}\left(S_{\lambda}\right)$ that maps to the natural image $s_{\lambda} \in F_{\lambda}\left(S_{\lambda}\right)$. The tensor product $S$ of all the $S_{\lambda}$ over $R$ (which is the filtered direct limit of the finite tensor products) is again faithfully flat of ind-finite presentation over $R$. Since $\left\{t_{\lambda}\right\} \in \prod G_{\lambda}(S)$ maps to $\left\{s_{\lambda}\right\} \in \prod F_{\lambda}(S)$, the morphism $\prod G_{\lambda} \rightarrow \prod F_{\lambda}$ is surjective. The same proof works for $\operatorname{Set}\left(k_{\text {proet }}^{\text {perf }}\right)$ and $\operatorname{Set}\left(k_{\text {proet }}^{\text {indrat }}\right)$. (Ee) In either cases, the left exactness is obvious.

For the right exactness of $\mathrm{PAlg} / k \rightarrow \mathrm{Ab}\left(k_{\text {profppf }}^{\text {perf }}\right)$, let $0 \rightarrow A \rightarrow B \rightarrow C \rightarrow 0$ be an exact sequence in $\mathrm{PAlg} / k$. To show that $B \rightarrow C$ is a surjection in $\mathrm{Ab}\left(k_{\text {profppf }}^{\text {perf }}\right)$, it is enough to show that $B \rightarrow C$ is faithfully flat of profinite presentation. Suppose first that $A \in \mathrm{Alg} / k$. Let $\left\{B_{\mu}\right\}$ be a filtered decreasing family of proalgebraic subgroups of $B$ such that $B \stackrel{\sim}{\rightarrow} \lim B / B_{\mu}$ (i.e., $\cap B_{\mu}=0$; Ser60, $\S 2.5$, Cor. 3 to Prop. 10]) and $B / B_{\mu} \in \mathrm{Alg} / k$ for all $\mu$. Then $A \cap B_{\mu}=0$ for some $\mu$ since $\mathrm{Alg} / k$ is artinian [Ser60, $\S 1.3$, Prop. 6]. This implies $B=\left(B / B_{\mu}\right) \times_{C / B_{\mu}} C$. We have an exact sequence $0 \rightarrow A \rightarrow B / B_{\mu} \rightarrow C / B_{\mu} \rightarrow 0$ in $\mathrm{Alg} / k$. Hence $B / B_{\mu} \rightarrow C / B_{\mu}$ is faithfully flat of finite presentation. Its base change $B \rightarrow C$ is thus faithfully flat of finite presentation. Suppose next that $A \in \mathrm{PAlg} / k$. Write $A=\lim A / A_{\lambda}$ with $A / A_{\lambda} \in \mathrm{Alg} / k$ as above. Then $B \rightarrow C$ can be written as the filtered inverse limit of the morphisms $B / A_{\lambda} \rightarrow C$. The kernel of $B / A_{\lambda} \rightarrow C$ is $A / A_{\lambda} \in \operatorname{Alg} / k$. Hence the previous case shows that $B / A_{\lambda} \rightarrow C$ is faithfully flat of finite presentation.

By (ㄷ) (exactness of pushforward), the exactness of $\mathrm{PAlg} / k \rightarrow \mathrm{Ab}\left(k_{\text {proet }}^{\text {indrat }}\right)$ is reduced to that of $\mathrm{PAlg} / k \rightarrow \mathrm{Ab}\left(k_{\text {proet }}^{\text {perf }}\right)$. To show this final statement, let $0 \rightarrow$ $A \rightarrow B \rightarrow C \rightarrow 0$ be an exact sequence in $\mathrm{PAlg} / k$. Suppose that we are given an $X$-valued point of $C$, where $X=\operatorname{Spec} R$ with $R \in k^{\text {perf }}$. By the exactness of 
$\mathrm{PAlg} / k \rightarrow \mathrm{Ab}\left(k_{\text {profppf }}^{\text {perf }}\right)$, the fiber $Y$ of the morphism $B \rightarrow C$ over the $X$-valued point $X \rightarrow C$ is a pro-fppf torsor for $A$ over $X$. We want to show that $Y \rightarrow X$ admits a section pro-étale locally. By [BS15, Cor. 2.2.14], there exists a pro-étale cover $X^{\prime} \rightarrow X$ such that $X^{\prime}$ is w-strictly local [BS15, Def. 2.2.1]. In particular, every étale cover of $X^{\prime}$ admits a section. Therefore we may assume that $X$ itself is w-strictly local. We show that $Y \rightarrow X$ then admits a section. Let $\left\{A_{\lambda}\right\}$ be a filtered decreasing family of proalgebraic subgroups of $A$ such that $A \stackrel{\sim}{\rightarrow} \underset{\lim }{\longleftarrow} A / A_{\lambda}$ (i.e., $\cap A_{\lambda}=0$ ) and $A / A_{\lambda} \in \mathrm{Alg} / k$ for all $\lambda$. Then we have $Y \stackrel{\sim}{\rightarrow} \lim Y / A_{\lambda}$ and $Y / A_{\lambda}$ is a pro-fppf torsor for $A / A_{\lambda}$ over $X$. Since $A / A_{\lambda}$ is quasi-algebraic, we know that $H^{1}\left(X_{\text {profppf }}, A / A_{\lambda}\right)=H^{1}\left(X_{\text {et }}, A / A_{\lambda}\right)$ by [Suz13, Cor. 3.3.3]. Therefore $Y / A_{\lambda}$ is an étale torsor for $A / A_{\lambda}$ over $X$. For each $\lambda$, this torsor is trivial since $X$ is w-strictly local. In this situation, if $R$ is an algebraically closed field, then Ser60, 2.3, Prop. 2] or [SY12, Lem. 3.7] says that $Y \rightarrow X=\operatorname{Spec} R$ admits a section. The arguments there actually work for any w-strictly local $X$.

(If) The higher $R \mathrm{lim}$ vanishes on $\mathrm{PAlg} / k$. The functors from $\mathrm{PAlg} / k$ to each of the categories preserves $R$ lim by the previous two assertions and [Nee01, Lem. A.3.2].

(g) The statement for the morphism $g$ on the ind-rational sites follows from that for the morphism $g$ on the perfect sites by (드. The cases $A \in \mathrm{IPAlg} / k, \mathrm{IAlg} / k$ are reduced to the cases $A \in \mathrm{PAlg} / k, \mathrm{Alg} / k$, respectively, by (回). Note that $R \lim _{\lambda}$ commutes with $R f_{*}$. With this and (f) , the case $A \in \mathrm{PAlg} / k$ is reduced to the case $A \in \mathrm{Alg} / k$. Summing all up, we are reduced to the case $A \in \mathrm{LAlg} / k$. This case is [Suz13, Cor. 3.3.3].

For the rest of the paper, we denote the object $R \Gamma\left(k_{\text {proet }}^{\text {indrat }} / k^{\prime}, f_{*} A\right)$ appearing in (c) simply by

$$
R \Gamma\left(k_{\text {proet }}^{\prime}, A\right)
$$

This is the same as the cohomology of the small pro-étale site Spec $k_{\text {proet }}^{\prime}$ with coefficients given by the restriction of $A$ to Spec $k_{\text {proet }}^{\prime}$, since big sites (Spec $k_{\text {proet }}^{\text {perf }}$ ) and small sites (Spec $k_{\text {proet }}^{\prime}$ ) have the same cohomology theory (Mil80, III, Rmk. $3.2])$. Its étale version $R \Gamma\left(k_{\mathrm{et}}^{\prime}, A\right)$ is used in a similar sense.

Note that the sites Spec $k_{\mathrm{et}}^{\text {indrat }}$ and Spec $k_{\text {proet }}^{\text {indrat }}$ are not coherent. If $x$ and $y$ are the generic points of irreducible varieties over $k$, then their fiber product as a sheaf on these sites is given by the disjoint union of the points (identified with the Spec of their residue fields) of the underlying set of the usual fiber product $x \times_{k} y$. This is infinite unless $x$ or $y$ is finite over $k$.

2.2. Generalities on derived categories of ind-procategories. A general reference on derived categories of indcategories is [KS06, Chap. 15]. We need to develop some more here. For a certain abelian category $\mathcal{A}$, we describe the derived Hom functor $R \operatorname{Hom}_{\operatorname{IP} \mathcal{A}}$ on the ind-procategory $\operatorname{IP} \mathcal{A}=\mathrm{I}(\mathrm{P} \mathcal{A})$ (i.e. the ind-category of the pro-category $\mathrm{P} \mathcal{A}$ ) in terms of $R \operatorname{Hom}_{\mathcal{A}}$. Recall that $\operatorname{Hom}_{\mathrm{IP} \mathcal{A}}$ is defined as the inverse limit of the direct limit of the inverse limit of the direct limit of $\operatorname{Hom}_{\mathcal{A}}$. Roughly speaking, the two inverse limits and the one Hom functor should be derived to get the required description of $R \operatorname{Hom}_{I P \mathcal{A}}$. This turns out to be quite complicated both notationally and mathematically. We organize it by introducing a relatively reasonable notation. In the next subsection, we will apply these results and notation to $\mathcal{A}=\mathrm{Alg} / k$ and $\mathrm{Ab}\left(k_{\text {proet }}^{\text {indrat }}\right)$. To clarify the argument, we generalize 
the situation and treat small abelian categories and Grothendieck categories in this subsection.

We need notation. Let $\mathcal{A}$ be an additive category. A filtered direct system $\left\{A_{\lambda}\right\}_{\lambda \in \Lambda} \in \mathrm{I} \mathcal{A}$ will occasionally be written as "lim" ${ }_{\lambda \in \Lambda} A_{\lambda}$. This is the direct limit of the $A_{\lambda}$ in I $\mathcal{A}$. If $\left\{A_{\lambda}\right\}_{\lambda \in \Lambda}$ is a family of objects of $\mathcal{A}$, then its direct sum in $\mathrm{I} \mathcal{A}$ (which is the filtered direct limit of finite sums) is denoted by " $\bigoplus$ " ${ }_{\lambda \in \Lambda} A_{\lambda}$. If $\mathcal{A}^{\prime}$ is a full additive subcategory of $\mathcal{A}$, then we denote by " $\bigoplus$ " $\mathcal{A}^{\prime}$ the full additive subcategory of I $\mathcal{A}$ consisting of objects of the form " $\bigoplus$ " ${ }_{\lambda \in \Lambda} A_{\lambda}$ with $A_{\lambda} \in \mathcal{A}^{\prime}$. A similar notation is applied to $\mathrm{P} \mathcal{A}$, for example, objects " $\lim _{\lambda}{ }_{\lambda} A_{\lambda}, " \prod^{\prime}{ }_{\lambda} A_{\lambda}$ of $\mathrm{P} \mathcal{A}$ and full additive subcategories " $\prod$ " $\mathcal{A}$ ' of $\mathrm{P} \mathcal{A}$.

Now let $\mathcal{A}$ be an abelian category. Assume that $\mathcal{A}$ is small. Then $\mathrm{P} \mathcal{A}$ has exact filtered inverse limits and a set of cogenerators given by objects of $\mathcal{A}$. Hence $\mathrm{P} \mathcal{A}$ is a co-Grothendieck category (i.e. the opposite of a Grothendieck category) and, in particular, has enough projectives. Therefore the Hom functor on $\mathrm{P} \mathcal{A}$ admits a derived functor $R \operatorname{Hom}_{\mathrm{P} \mathcal{A}}$. Its restriction to the bounded derived category of $\mathcal{A}$ is denoted by $R \operatorname{Hom}_{\mathcal{A}}$, namely we define

$$
R \operatorname{Hom}_{\mathcal{A}}(A, B):=R \operatorname{Hom}_{\mathcal{A}}(A, B) \quad \text { for } \quad A, B \in D^{b}(\mathcal{A}) .
$$

We do not claim that this $R \operatorname{Hom}_{\mathcal{A}}$ is the derived functor of $\operatorname{Hom}_{\mathcal{A}}$. By KS06, Thm. 15.3.1, (i)], the natural functor $D^{b}(\mathcal{A}) \rightarrow D^{b}(\mathrm{P} \mathcal{A})$ is fully faithful, hence

$$
H^{n} R \operatorname{Hom}_{\mathcal{A}}(A, B)=\operatorname{Ext}_{\mathcal{A}}^{n}(A, B) \quad \text { for } \quad A, B \in \mathcal{A},
$$

where the right-hand side is the usual Ext functor for the abelian category $\mathcal{A}$. Therefore if the derived functor of $\operatorname{Hom}_{\mathcal{A}}$ on the bounded derived category exists, then the morphism of functors from this derived functor to the above $R \operatorname{Hom}_{\mathcal{A}}$ defined by universality is an isomorphism. In general, we do not assume this. If $A=" \varliminf^{\prime}{ }_{\lambda} A_{\lambda} \in \mathrm{P} \mathcal{A}$ and $B \in \mathcal{A}$, then

$$
\operatorname{Ext}_{\mathrm{P} \mathcal{A}}^{n}(A, B)=\underset{\lambda}{\lim _{\lambda}} \operatorname{Ext}_{\mathcal{A}}^{n}\left(A_{\lambda}, B\right)
$$

for all $n$ by [KS06, Cor. 15.3.9]. We express this result by the equality

$$
R \operatorname{Hom}_{\mathrm{P} \mathcal{A}}(A, B)=\underset{\lambda}{\lim } R \operatorname{Hom}_{\mathcal{A}}\left(A_{\lambda}, B\right),
$$

which is intuitive and convenient, but may be confusing since the $\lim _{\rightarrow}$ in the righthand side is not a direct limit in the triangulated category $D(\mathrm{Ab})$.

Next we want to treat the case that $B$ also is a pro-object and, more generally, the case $A, B \in \mathrm{IP} \mathcal{A}$. An object $A \in \mathrm{IP} \mathcal{A}$ can be written as $A=\stackrel{\text { "lim" }}{\lambda \in \Lambda} A_{\lambda}$, where each $A_{\lambda} \in \mathrm{P} \mathcal{A}$ can be written as $A_{\lambda}=$ "lim" ${\stackrel{\lambda^{\prime} \in \Lambda_{\lambda}^{\prime}}{ }} A_{\lambda \lambda^{\prime}}$ with $A_{\lambda \lambda^{\prime}} \underset{\mathcal{A}}{\longrightarrow}$. Note that the morphisms $A_{\lambda_{1}} \rightarrow A_{\lambda_{2}} \in \mathrm{P} \mathcal{A}$ for $\lambda_{1} \leq \lambda_{2} \in \Lambda$ are assumed as given, but no maps $\Lambda_{\lambda_{2}}^{\prime} \rightarrow \Lambda_{\lambda_{1}}^{\prime}$ between the index sets are assumed. The Hom functor for IP $\mathcal{A}$ is given by definition as

$$
\operatorname{Hom}_{\mathrm{IP} \mathcal{A}}(A, B)=\lim _{\lambda} \underset{\mu}{\lim } \underset{\mu_{\mu^{\prime}}}{\lim } \underset{\lambda^{\prime}}{\lim } \operatorname{Hom}_{\mathcal{A}}\left(A_{\lambda \lambda^{\prime}}, B_{\mu \mu^{\prime}}\right)
$$

for $A=$ "lim" ${ }_{\lambda}$ "lim" ${ }_{\lambda^{\prime}} A_{\lambda \lambda^{\prime}} \in \operatorname{IP} \mathcal{A}$ and $B=$ "lim" " "lim" ${ }_{\mu^{\prime}} B_{\mu \mu^{\prime}} \in \operatorname{IP} \mathcal{A}$, which is of the form

$$
\operatorname{Hom}_{\mathrm{IP} \mathcal{A}}: \operatorname{PI}\left(\mathcal{A}^{\mathrm{op}}\right) \times \operatorname{IP} \mathcal{A} \rightarrow \mathrm{Ab} .
$$

A general method to derive this type of functors is the following. 
Proposition (2.2.1). Let $\mathcal{A}, \mathcal{B}, \mathcal{C}$ be abelian categories and $F: \mathcal{A} \times \mathcal{B} \rightarrow \mathcal{C}$ an additive bifunctor that is left exact in both variables. Let $\mathcal{A}^{\prime}, \mathcal{B}^{\prime}, \mathcal{C}^{\prime}$ be full additive subcategories of $\mathcal{A}, \mathcal{B}, \mathcal{C}$, respectively, such that $F\left(\mathcal{A}^{\prime} \times \mathcal{B}^{\prime}\right) \subset \mathcal{C}^{\prime}$. Let $\mathrm{P} F: \mathrm{P} \mathcal{A} \times$ $\mathrm{PB} \rightarrow \mathrm{PC}, \operatorname{IP} F: \operatorname{IP} \mathcal{A} \times \operatorname{IPB} \rightarrow \operatorname{IPC}, \operatorname{PIP} F: \operatorname{PIP} \mathcal{A} \times \operatorname{PIPB} \rightarrow \operatorname{PIPC}$ etc. be the natural extensions of $F$.

(a) Assume that the pair $\left(\mathcal{A}^{\prime}, \mathcal{B}^{\prime}\right)$ is $F$-injective in the sense of [KS06. Def. 13.4.2] (which implies the existence of the derived functor of $F$ on the bounded below derived categories). Then $\left(\mathrm{I} \mathcal{A}^{\prime}, \mathrm{I} \mathcal{B}^{\prime}\right)$ is $\mathrm{I} F$-injective, and $\left(\mathrm{P} \mathcal{A}^{\prime}, \mathrm{P} \mathcal{B}^{\prime}\right)$ and ( $П " \mathcal{A}$ ', " $\prod$ " $\left.\mathcal{B}^{\prime}\right)$ are both $\mathrm{P} F$-injective. In particular, $\mathrm{I} F$ and $\mathrm{P} F$ admit derived functors on the bounded below derived categories. The diagrams

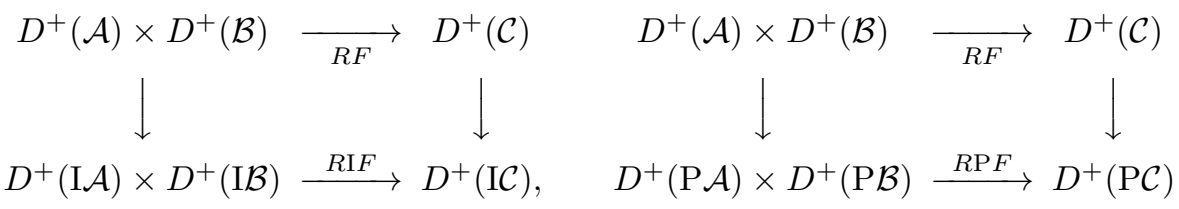

commute. We have natural isomorphisms $R^{n} \mathrm{I} F \cong \mathrm{I} R^{n} F$ and $R^{n} \mathrm{P} F \cong$ $\mathrm{P} R^{n} F$ of functors for all $n$.

(b) Assume that: $\mathcal{C}$ has products and exact filtered direct limits; $\mathcal{C}^{\prime}$ is closed by products and filtered direct limits; the subcategory $\mathcal{C}^{\prime} \subset \mathcal{C}$ is cogenerating, i.e., any object of $\mathcal{C}$ has an injection into an object of $\mathcal{C}^{\prime}$; and for any family of exact sequences $0 \rightarrow C_{\lambda}^{1} \rightarrow C_{\lambda}^{2} \rightarrow C_{\lambda}^{3} \rightarrow 0$ in $\mathcal{C}$ with $C_{\lambda}^{1}, C_{\lambda}^{2} \in \mathcal{C}^{\prime}$ for any $\lambda$, we have $C_{\lambda}^{3} \in \mathcal{C}^{\prime}$ for any $\lambda$ and the sequence $0 \rightarrow \prod C_{\lambda}^{1} \rightarrow \prod C_{\lambda}^{2} \rightarrow$ $\prod C_{\lambda}^{3} \rightarrow 0$ is exact. Then the sequence

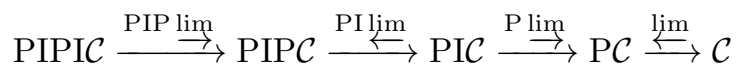

of functors restricts to the sequence

$$
\text { "॥ " }
$$

on the subcategories. Each category in the latter sequence is injective with respect to the functor that follows in the former sequence (i.e. " $\Pi$ " I " $\Pi$ " $\mathcal{C}^{\prime}$ is PIlim-injective etc.). In particular, the composite of the derived functors of the functors in the former sequence gives the derived functor of the "takeall-the-limits" functor

$$
\lim _{\longleftarrow} \mathrm{P} \underset{\longrightarrow}{\lim } \mathrm{PI} \underset{\longleftarrow}{ } \mathrm{PIP} \underset{\longrightarrow}{\longrightarrow} \mathrm{PIPIC} \rightarrow \mathcal{C} .
$$

(c) Under the assumptions of the previous two assertions, the composite

$$
\mathrm{PIPI} \mathcal{A} \times \mathrm{PIPI} \mathcal{B} \stackrel{\mathrm{PIPI} F}{\longrightarrow} \mathrm{PIPIC} \stackrel{\lim }{\longleftarrow} \mathrm{P} \lim _{\longrightarrow} \mathrm{PI} \lim _{\leftarrow} \mathrm{PIP} \stackrel{\lim }{\longrightarrow} \mathcal{C}
$$

admits a derived functor on the bounded below derived categories, which is given by the composite of the derived functors.

Recall that $\left(\mathcal{A}^{\prime}, \mathcal{B}^{\prime}\right)$ being $F$-injective means that: for any bounded below complex $A$ in $\mathcal{A}$, there is a quasi-isomorphism $A \rightarrow A^{\prime}$ to a bounded below complex $A^{\prime}$ in $\mathcal{A}^{\prime}$; for any bounded below complex $B$ in $\mathcal{B}$, there is a quasi-isomorphism $B \rightarrow B^{\prime}$ to a bounded below complex $B^{\prime}$ in $\mathcal{B}^{\prime}$; and $F\left(A^{\prime}, B^{\prime}\right)$ is an exact complex if $A^{\prime}$ (resp. $B^{\prime}$ ) is a bounded below complex in $\mathcal{A}^{\prime}$ (resp. $\mathcal{B}^{\prime}$ ) such that either $A^{\prime}$ or $B^{\prime}$ is exact. In this situation, according to [KS06, §13.4], the derived functor

$$
R F: D^{+}(\mathcal{A}) \times D^{+}(\mathcal{B}) \rightarrow D^{+}(\mathcal{C})
$$


of the two-variable functor $F$ is defined by $R F(A, B)=F\left(A^{\prime}, B^{\prime}\right)$, where $A \stackrel{\sim}{\rightarrow} A^{\prime}$ is a quasi-isomorphism to a bounded below complex in $A^{\prime}$ and $B \stackrel{\sim}{\rightarrow} B^{\prime}$ is a quasiisomorphism to a bounded below complex in $B^{\prime}$. We need to replace the both variables at the same time. In the proposition, we chose a pro-ind-pro-indcategory as an example. There is nothing special about this choice. There is a corresponding statement for any finite sequence of P's and I's.

Proof. (国) This is nothing but a two-variable version of KS06, Prop. 15.3.2, 15.3.7]. We merely indicate what should be modified from the original single-variable version. Let $\tilde{\mathcal{A}}^{\prime}$ be the full subcategory of $\mathrm{I} \mathcal{A}$ consisting of objects $A \in \mathrm{I} \mathcal{A}$ such that $\mathrm{I} R^{n} F(A, B)=0$ for any $B \in \mathrm{I}^{\prime}$ and $n \geq 1$. Let $\tilde{\tilde{\mathcal{B}}}^{\prime}$ be the full subcategory of $\mathrm{I} \mathcal{B}$ consisting of objects $B \in \mathrm{I} \mathcal{B}$ such that $\operatorname{I}^{n} F(A, B)=0$ for any $A \in \tilde{\mathcal{A}}^{\prime}$ and $n \geq 1$. Then exactly in the same manner as [KS06, loc.cit.], we can show that $\tilde{\mathcal{A}}^{\prime} \times \tilde{\tilde{\mathcal{B}}}^{\prime}$ contains $\mathrm{I} \mathcal{A}^{\prime} \times \mathrm{I} \mathcal{B}^{\prime}$ and that $\left(\tilde{\mathcal{A}}^{\prime}, \tilde{\tilde{\mathcal{B}}}^{\prime}\right)$ is $\mathrm{I} F$-injective. We can deduce from this that $\left(\mathrm{I} \mathcal{A}^{\prime}, \mathrm{I} \mathcal{B}^{\prime}\right)$ is $\mathrm{I} F$-injective. A similar argument for the pro version implies that $\left(\mathrm{P} \mathcal{A}^{\prime}, \mathrm{P} \mathcal{B}^{\prime}\right)$ is $\mathrm{P} F$-injective. Since " $\Pi$ " $\mathcal{A}^{\prime}$ is cogenerating in $\mathrm{P} \mathcal{A}^{\prime}$, we know that ("П" $\left.\mathcal{A}^{\prime}, " \prod " \mathcal{B}^{\prime}\right)$ is $\mathrm{P} F$-injective. We omit the details.

(b) The assumptions imply that the subcategory " $\Pi$ " $\mathcal{C}^{\prime} \subset \mathrm{PC}$ is injective with respect to the functor $\lim _{\mathrm{P}} \mathrm{PC} \rightarrow \mathcal{C}$ by [KS06, Prop. 13.3.15]. The subcategory $\mathrm{IC}^{\prime} \subset \mathrm{IC}$ is cogenerating by [KS06, Thm. 15.2.5], hence injective with respect to the exact functor $\lim : \mathrm{IC} \rightarrow \mathcal{C}$. These imply the rest of the statement by an iterated usage of $[\overrightarrow{\mathrm{KS} 06}$, Prop. 15.3.2, 15.3.7] and the theorem on derived functors of composition [KS06, Prop. 13.3.13].

(C) This follows from the previous two assertions and [KS06, Prop. 13.3.13].

Hence, in the situation of the proposition, we have

$$
\begin{aligned}
& R\left(\lim _{\longleftarrow} \mathrm{P} \underset{\longrightarrow}{\lim } \mathrm{PI} \lim _{\longleftarrow} \mathrm{PIP} \underset{\mathrm{lim}}{\longrightarrow} \mathrm{PIPI} F\right)(A, B) \\
& =R \lim _{\longleftarrow} \mathrm{P} \underset{\longrightarrow}{l} R \mathrm{PI} \varliminf_{\longleftarrow} \mathrm{PIP} \underset{\lim }{\longrightarrow} R \operatorname{PIPIF}(A, B) \in D^{+}(\mathcal{C})
\end{aligned}
$$

for $A \in D^{+}(\mathrm{PIPI} \mathcal{A}), B \in D^{+}(\mathrm{PIPI} \mathcal{B})$. Again, an intuitive and convenient but less rigorous way to denote the painful right-hand side (when $A \in \operatorname{PIPI} \mathcal{A}, B \in \mathrm{PIPI} \mathcal{B}$ ) is

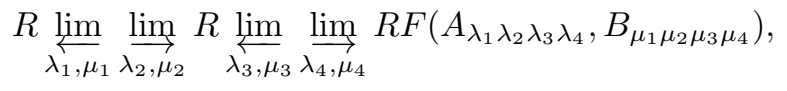

where

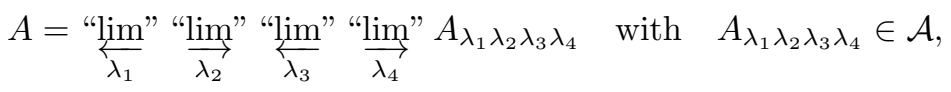

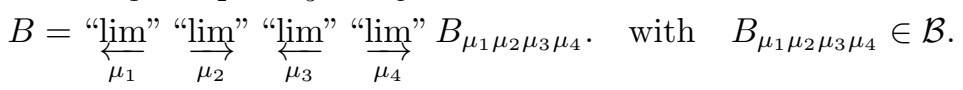

What is rigorously true about this notation is that there are two spectral sequences. One (for fixed $\lambda_{1}, \lambda_{2}, \mu_{1}, \mu_{2}$ ) has $E_{2}^{i j}$-terms given by

$$
R^{i} \underset{\lambda_{3}, \mu_{3}}{\lim _{\lambda_{4}, \mu_{4}}} \lim ^{j} F\left(A_{\lambda_{1} \lambda_{2} \lambda_{3} \lambda_{4}}, B_{\mu_{1} \mu_{2} \mu_{3} \mu_{4}}\right)
$$


converging to $R^{i+j} \bar{F}\left(A_{\lambda_{1} \lambda_{2}}, B_{\mu_{1} \mu_{2}}\right)$, where

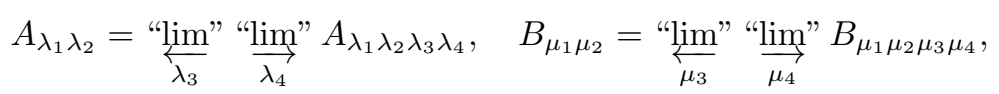

$\bar{F}=\lim _{\longleftarrow} P \underset{\lim }{\longrightarrow}$ PIF, i.e., $\quad \bar{F}\left(A_{\lambda_{1} \lambda_{2}}, B_{\mu_{1} \mu_{2}}\right)=\lim _{\lambda_{3}, \mu_{3}} \underset{\lambda_{4}, \mu_{4}}{\lim _{4}} F\left(A_{\lambda_{1} \lambda_{2} \lambda_{3} \lambda_{4}}, B_{\mu_{1} \mu_{2} \mu_{3} \mu_{4}}\right)$.

Varying $\lambda_{1}, \lambda_{2}, \mu_{1}, \mu_{2}$, this spectral sequence takes values in PIC. The other has $E_{2}^{i j}$-terms given by

$$
R^{i} \underset{\lambda_{1}, \mu_{1}}{\lim _{\lambda_{2}, \mu_{2}}} R^{j} \bar{F}\left(A_{\lambda_{1} \lambda_{2}}, B_{\mu_{1} \mu_{2}}\right)
$$

converging to $R^{i+j} \overline{\bar{F}}(A, B)$, where

$$
\begin{aligned}
& \overline{\bar{F}}=\lim _{\longleftrightarrow} \mathrm{P} \underline{\lim } \mathrm{PI} \lim _{\longleftarrow} \mathrm{PIP} \underset{\lim }{\longrightarrow} \mathrm{PIPI} F, \quad \text { i.e., } \\
& \overline{\bar{F}}(A, B)=\lim _{\lambda_{1}, \mu_{1}} \underset{\lambda_{2}, \mu_{2}}{\lim _{\lambda_{3}, \mu_{3}}} \underset{\lambda_{4}, \mu_{4}}{\lim } F\left(A_{\lambda_{1} \lambda_{2} \lambda_{3} \lambda_{4}}, B_{\mu_{1} \mu_{2} \mu_{3} \mu_{4}}\right) .
\end{aligned}
$$

A special case we use below is where $A \in \operatorname{PI} \mathcal{A}$ and $B \in \operatorname{IPB}$. Here we embed $\mathrm{PI} \mathcal{A}$ into PIPI $\mathcal{A}$ by adding $I$ and $P$ in the middle and IP $\mathcal{B}$ into PIPI $\mathcal{B}$ by adding $\mathrm{P}$ from the left and I from the right. These embeddings are exact functors. The first one takes the subcategory " $\prod$ " I $\mathcal{A}^{\prime}$ into " $\prod$ " I " $\prod$ " $I \mathcal{A}$ '. The second takes the subcategory I " $\prod$ " $\mathcal{B}^{\prime}$ into " $\prod$ " I " $\prod$ " $\mathrm{I} \mathcal{B}^{\prime}$. Hence the derived functor of the restriction of $\overline{\bar{F}}$ to $\mathrm{PI} \mathcal{A} \times \operatorname{IP} \mathcal{A}$ is the restriction of $R \overline{\bar{F}}$ to $D^{+}(\mathrm{PI} \mathcal{A}) \times D^{+}(\operatorname{IP} \mathcal{A})$, and we have

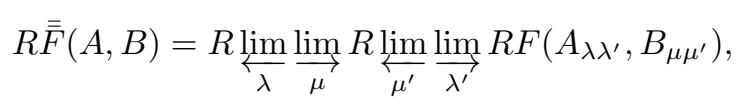

where $A=$ "lim" " ${ }_{\lambda} \longrightarrow{ }_{\lambda^{\prime}} A_{\lambda \lambda^{\prime}} \in \mathrm{PI} \mathcal{A}, B=$ "lim" "lim" ${ }_{\mu^{\prime}} B_{\mu \mu^{\prime}} \in \mathrm{IP \mathcal {B }}$. Similar observations apply to adding more or less P's and/or I's in different places.

Proposition (2.2.2). Let $\mathcal{A}$ be a small abelian category. Then we have natural fully faithful embeddings

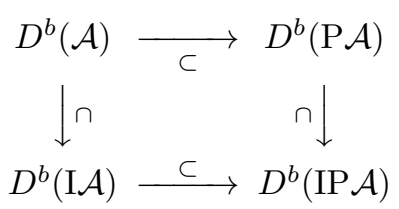

of triangulated categories. We have

$$
R \operatorname{Hom}_{\mathrm{P} \mathcal{A}}(A, B)=R \lim _{\mu} \underset{\lambda}{\lim _{\lambda}} R \operatorname{Hom}_{\mathcal{A}}\left(A_{\lambda}, B_{\mu}\right)
$$

for $A=\lim { }_{\lambda} A_{\lambda} \in \mathrm{P} \mathcal{A}$ and $B=\lim ^{\longleftarrow}{ }_{\mu} B_{\mu} \in \mathrm{P} \mathcal{A}$,

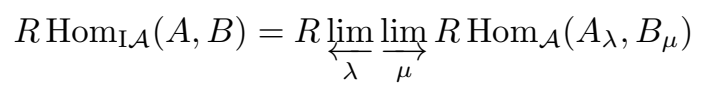

for $A=\stackrel{\longrightarrow}{\longrightarrow}{ }_{\lambda} A_{\lambda} \in \mathrm{I} \mathcal{A}$ and $B=\lim { }_{\mu} B_{\mu} \in \mathrm{I} \mathcal{A}$, and

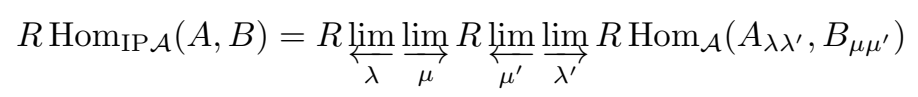

for $A=\stackrel{\text { lim }}{\longrightarrow}{ }_{\lambda}$ " "im ${ }_{\lambda^{\prime}}, A_{\lambda \lambda^{\prime}} \in \operatorname{IP} \mathcal{A}$ and $B=\stackrel{\text { "im }}{\longrightarrow}{ }_{\mu} \stackrel{\text { "im }}{\longleftarrow}{ }_{\mu^{\prime}} B_{\mu \mu^{\prime}} \in \operatorname{IP} \mathcal{A}$. 
Proof. The fully faithful embedding $D^{b}(\mathcal{A}) \hookrightarrow D^{b}(\mathrm{P} \mathcal{A})$ has already been mentioned ([KS06, Thm. 15.3.1 (i)]). The same implies the fully faithfulness of $D^{b}(\mathcal{A}) \hookrightarrow$ $D^{b}(\mathrm{I} \mathcal{A})$ and $D^{b}(\mathrm{P} \mathcal{A}) \hookrightarrow D^{b}(\operatorname{IP} \mathcal{A})$. The exactness of the embedding $\operatorname{I} \mathcal{A} \hookrightarrow \operatorname{IP} \mathcal{A}$ yields a morphism of functors from $R \operatorname{Hom}_{\mathrm{I} \mathcal{A}}$ to $R \operatorname{Hom}_{\mathrm{IP} \mathcal{A}}$ restricted to $D^{b}(\mathrm{I} \mathcal{A})$. This will turn out to be an isomorphism and hence the fully faithfulness of $D^{b}(\mathrm{I} \mathcal{A}) \rightarrow$ $D^{b}(\operatorname{IP} \mathcal{A})$ will follow once we compute these $R$ Hom functors and verify the stated formulas.

We compute $R \operatorname{Hom}_{\mathrm{IP} \mathcal{A}}$. Let $\mathcal{B}=(\mathrm{P} \mathcal{A})^{\mathrm{op}}, \mathcal{B}^{\prime}$ its full subcategory of injectives (= the opposite of projectives of $\mathrm{P} \mathcal{A}$ ) and $\mathcal{C}=\mathcal{C}^{\prime}=\mathrm{Ab}$. We will apply the observations we made before the proposition for

$$
F:=\operatorname{Hom}_{\mathrm{P} \mathcal{A}}:(\mathrm{P} \mathcal{A})^{\mathrm{op}} \times \mathcal{A}=: \mathcal{B} \times \mathcal{A} \rightarrow \mathcal{C}=\mathrm{Ab}
$$

and

$$
\lim _{\longleftarrow} \mathrm{P} \lim _{\longrightarrow} \mathrm{PI} \lim _{\longleftarrow} \mathrm{PIP} F=\operatorname{Hom}_{\mathrm{IP} \mathcal{A}}: \mathrm{PB} \times \mathrm{IP} \mathcal{A} \rightarrow \mathrm{PIPC} \rightarrow \mathcal{C}
$$

(the $\mathrm{P}$ in $\mathrm{PB}$ corresponds to the left $\mathrm{P}$ in $\mathrm{PIPC}$ and the IP in IP $\mathcal{A}$ the right IP in PIPC). The pair $\left(\mathcal{B}^{\prime}, \mathcal{A}\right)$ is $F$-injective. Obviously $\mathcal{C}=\mathcal{C}^{\prime}=$ Ab satisfies the conditions of (2.2.1) (b). Hence we have

$$
\begin{aligned}
& R \operatorname{Hom}_{\mathrm{IP} \mathcal{A}}(A, B)=R \underset{\lambda}{\lim _{\lambda}} \underset{\mu}{\lim } R \underset{\mu^{\prime}}{\lim } R \operatorname{Hom}_{\mathrm{P} \mathcal{A}}\left({\underset{\lambda^{\prime}}{\left(\lim ^{\prime}\right.}} A_{\lambda \lambda^{\prime}}, B_{\mu \mu^{\prime}}\right) \\
& =R \underset{\lambda}{\lim _{\lambda}} \underset{\mu}{\lim } R \underset{\mu^{\prime}}{\lim _{\lambda^{\prime}}} \underset{\lim _{1}}{\longrightarrow} R \operatorname{Hom}_{\mathcal{A}}\left(A_{\lambda \lambda^{\prime}}, B_{\mu \mu^{\prime}}\right)
\end{aligned}
$$

for $A=$ "lim" ${ }_{\lambda}$ "lim" ${ }_{\lambda^{\prime}} A_{\lambda \lambda^{\prime}} \in \operatorname{IP} \mathcal{A}$ and $B=$ "lim" " "lim" ${ }_{\mu^{\prime}} B_{\mu \mu^{\prime}} \in \operatorname{IP} \mathcal{A}$.

Since $D^{b}(\mathrm{P} \mathcal{A}) \hookrightarrow D^{b}(\mathrm{IP} \mathcal{A})$ is fully faithful, this also verifies, by restriction, the stated formula for $R \operatorname{Hom}_{\mathrm{P} \mathcal{A}}$. Dualizing, this in turn verifies the stated formula for $R \operatorname{Hom}_{I \mathcal{A}}$. This completes the proof.

Note that this proposition in particular implies that the restriction of $R \mathrm{Hom}_{\mathrm{I} \mathcal{A}}$ to $D^{b}(\mathcal{A})$ agrees with $R \operatorname{Hom}_{\mathcal{A}}$, which was originally defined as the restriction of $R \operatorname{Hom}_{\mathrm{P} \mathcal{A}}$. The proposition is also true for a Grothendieck category $\mathcal{A}$. We omit the proof as we do not need this case.

What we need to know about Grothendieck categories is when direct and derived inverse limits commute with $R$ Hom in the both variables. The following shows what is true in general and what should be checked in specific cases.

Proposition (2.2.3). Let $\mathcal{A}, \mathcal{C}$ be Grothendieck categories and $F: \mathcal{A}^{\mathrm{op}} \times \mathcal{A} \rightarrow \mathcal{C}$ an additive bifunctor that is left exact in both variables. Let $\mathcal{C}^{\prime}$ be a full additive subcategory of $\mathcal{C}$. Assume that the functor $F(\cdot, I): \mathcal{A}^{\mathrm{op}} \rightarrow \mathcal{C}$ for any injective $I \in \mathcal{A}$ is exact with image contained in $\mathcal{C}^{\prime}$. Assume also that $F$ commutes with filtered inverse limits. Assume finally that $\mathcal{C}^{\prime} \subset \mathcal{C}$ satisfies the conditions of (2.2.1) 
(b). Then we have canonical morphisms and isomorphisms

$$
\begin{aligned}
& R \underset{\lambda}{\lim _{\lambda}} \underset{\mu}{\lim } R \underset{\mu^{\prime}}{\lim } \underset{\lambda^{\prime}}{\lim } R F\left(A_{\lambda \lambda^{\prime}}, B_{\mu \mu^{\prime}}\right)
\end{aligned}
$$

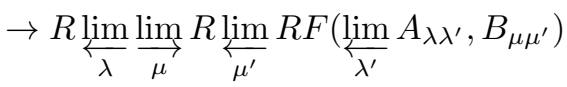

$$
\begin{aligned}
& =R \lim _{\lambda} \underset{\mu}{\lim } R F(\underbrace{\lim }_{\lambda^{\prime}} A_{\lambda \lambda^{\prime}}, R \underset{\mu^{\prime}}{\lim } B_{\mu \mu^{\prime}})
\end{aligned}
$$

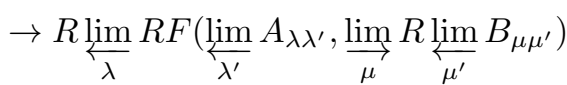

$$
\begin{aligned}
& =R F\left(\underset{\lambda}{\lim } \underset{\lambda^{\prime}}{\lim } A_{\lambda \lambda^{\prime}}, \underset{\mu}{\lim } R \underset{\mu^{\prime}}{\lim } B_{\mu \mu^{\prime}}\right)
\end{aligned}
$$

in $D^{+}(\mathcal{C})$ for any $A=\stackrel{\text { "lim }}{\longrightarrow}{ }_{\lambda}$ " "Lim ${ }_{\lambda^{\prime}} A_{\lambda \lambda^{\prime}} \in \operatorname{IP} \mathcal{A}$ and $B=\stackrel{\text { "im }}{\longrightarrow}{ }_{\mu} \stackrel{\text { "lim }}{\longleftarrow}{ }_{\mu^{\prime}} B_{\mu \mu^{\prime}} \in$ $\operatorname{IP} \mathcal{A}$.

Note that the $R \lim _{\mu^{\prime}}$ in the displayed equations are the derived ones but the $\lim _{\lambda^{\prime}}$ are not, so that all the variables lie in $D^{-}(\mathcal{A})^{\mathrm{op}} \times D^{+}(\mathcal{A})$ where $R F$ is defined.

Proof. In the rigorous terms, the morphisms and isomorphisms to be constructed are

$$
\begin{aligned}
& R \lim _{\longleftarrow} \mathrm{P} \underset{\longrightarrow}{\lim } R \mathrm{PI} \varliminf_{\leftarrow} \mathrm{PIP} \underset{\lim }{\longrightarrow} R \operatorname{PIPI} F(A, B) \\
& \rightarrow R \lim _{\longleftarrow} \mathrm{P} \lim _{\longrightarrow} R \mathrm{PI} \lim _{\longleftarrow} R \mathrm{PIP} F\left(\mathrm{I} \lim _{\longleftarrow} A, B\right) \\
& =R \varliminf_{\longleftarrow} \mathrm{P} \lim _{\longrightarrow} R \mathrm{PI} F\left(\mathrm{I} \varliminf_{\longleftarrow} A, R \mathrm{I} \varliminf_{\longleftarrow} B\right)
\end{aligned}
$$

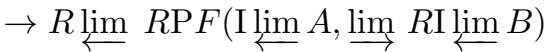

$$
\begin{aligned}
& =R F\left(\underline{\lim } \mathrm{I} \lim _{\longleftarrow} A, \underline{\lim } R \mathrm{I} \lim _{\longleftarrow} B\right)
\end{aligned}
$$

for $A, B \in \mathrm{IP} \mathcal{A}$. It suffices to construct a morphism

$$
\mathrm{PIP} \underset{\longrightarrow}{\longrightarrow} R \operatorname{PIPI} F(A, B) \rightarrow R \mathrm{PIP} F\left(\operatorname{I} \lim _{\longleftarrow} A, B\right) \quad \text { in } \quad D^{+}(\mathrm{PIPC})
$$

for $A \in \operatorname{IP} \mathcal{A}$ and $B \in D^{+}(\operatorname{IP} \mathcal{A})$, an isomorphism

$$
R \mathrm{PI} \lim _{\leftarrow} R \operatorname{PIP} F(A, B)=R \operatorname{PI} F\left(A, R \operatorname{I} \varliminf_{\leftarrow} B\right) \quad \text { in } \quad D^{+}(\mathrm{PIC})
$$

for $A \in D^{-}(\mathrm{I} \mathcal{A}), B \in D^{+}(\mathrm{IP} \mathcal{A})$, a morphism

$$
\mathrm{P} \underset{\longrightarrow}{\longrightarrow} R \operatorname{PI} F(A, B) \rightarrow R \mathrm{P} F\left(A, \lim _{\longrightarrow} B\right) \quad \text { in } \quad D^{+}(\mathrm{PC})
$$

for $A \in D^{-}(\mathrm{I} \mathcal{A}), B \in D^{+}(\mathrm{I} \mathcal{A})$, and an isomorphism

$$
R \lim _{\longleftarrow} R \mathrm{P} F(A, B)=R F\left(\lim _{\longrightarrow} A, B\right) \quad \text { in } \quad D^{+}(\mathcal{C})
$$

for $A \in D^{-}(\mathrm{I} \mathcal{A}), B \in D^{+}(\mathcal{A})$.

We construct the first morphism. We fix $A \in \operatorname{IP} \mathcal{A}$. Let $\mathcal{A}^{\prime} \subset \mathcal{A}$ be the full subcategory of injectives. Then $\left(\mathcal{A}^{\mathrm{op}}, \mathcal{A}^{\prime}\right)$ is $F$-injective by assumption. Hence $\left((\mathrm{IP} \mathcal{A})^{\mathrm{op}}, \mathrm{IP} \mathcal{A}^{\prime}\right)$ is PIPI $F$-injective by (2.2.1) (国). This implies that $\operatorname{RPIPI} F(A, B)$ (which is a priori calculated by resolving $A$ and $B$ at the same time) is the value at $B$ of the derived functor of the single-variable functor $\operatorname{PIPI} F(A, \cdot)([\mathrm{KS} 06$, Cor. 13.4.5]). Similarly $R \operatorname{PIP} F\left(\operatorname{I} \lim _{\longleftarrow} A, B\right)$ is the value at $B$ of the derived functor of $\mathrm{PIP} F(\mathrm{I} \lim A, \cdot)$. Hence we need to construct a morphism

$$
\mathrm{PIP} \underset{\lim }{\longrightarrow} R \operatorname{PIPI} F(A, \cdot) \rightarrow R \operatorname{PIP} F\left(\operatorname{I} \lim _{\longleftarrow} A, \cdot\right)
$$


of functors $D^{+}(\mathrm{IP} \mathcal{A}) \rightarrow D^{+}(\mathrm{PIPC})$. Since $\mathrm{PIP} \underline{\lim }: \mathrm{PIPIC} \rightarrow \mathrm{PIPC}$ is exact, we have

$$
\mathrm{PIP} \stackrel{\lim }{\longrightarrow} \operatorname{PIPI} F(A, \cdot)=R(\mathrm{PIP} \stackrel{\lim }{\longrightarrow} \operatorname{PIPI} F)(A, \cdot) .
$$

Deriving the natural morphism

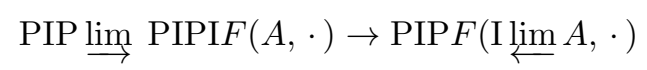

of functors IP $\mathcal{A} \rightarrow$ PIPC , we get the required morphism of derived functors.

We construct the second isomorphism. Let $\mathcal{A}^{\prime} \subset \mathcal{A}$ be the full subcategory of injectives. Since $F$ commute with filtered inverse limits, we have a commutative diagram

$$
\begin{aligned}
& (\mathrm{I} \mathcal{A})^{\mathrm{op}} \times \operatorname{IP} \mathcal{A} \underset{\mathrm{PIP} F}{\longrightarrow} \mathrm{PIPC} \\
& \downarrow \operatorname{id} \times \operatorname{Ilim} \underset{\leftarrow}{\operatorname{PI}} \underset{\leftarrow}{\leftarrow} \downarrow \\
& (\mathrm{I} \mathcal{A})^{\mathrm{op}} \times \mathrm{I} \mathcal{A} \stackrel{\mathrm{PI} F}{\longrightarrow} \mathrm{PIC} .
\end{aligned}
$$

This diagram restricts to the diagram

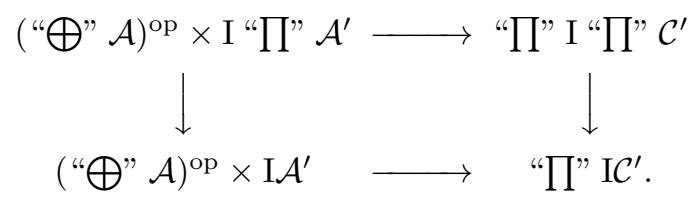

of full subcategories since $F\left(\mathcal{A}^{\mathrm{op}} \times \mathcal{A}^{\prime}\right) \subset \mathcal{C}^{\prime}$ and products of injectives are injective. The category ( $\bigoplus$ " $\mathcal{A}$ ) ${ }^{\mathrm{op}} \times I$ " $\prod$ " $\mathcal{A}$ ' is injective with respect to both $\operatorname{PIP} F$ and id $\times \mathrm{Il} \varliminf_{\text {by }}$ (2.2.1). Therefore the above commutative diagram derives to a commutative diagram of the derived functors on the derived categories. This gives the required isomorphism.

We construct the third morphism. Consider the following (non-commutative) diagram:

$$
\begin{aligned}
& (\mathrm{I} \mathcal{A})^{\mathrm{op}} \times \mathrm{I} \mathcal{A} \underset{\mathrm{PI} F}{\longrightarrow} \mathrm{PIC} \\
& \downarrow \operatorname{id} \times \lim _{\rightarrow} \stackrel{\mathrm{Plim}}{\rightarrow} \downarrow \\
& (\mathrm{I} \mathcal{A})^{\mathrm{op}} \times \mathcal{A} \stackrel{\mathrm{P} F}{\longrightarrow} \mathrm{PC} .
\end{aligned}
$$

This gives two functors from the left upper term to the right lower term. There is a natural morphism of functors from the one factoring through the right upper term to the one factoring through the left lower term. Since the right vertical arrow is an exact functor, this morphism of functors induces a morphism of functors in the derived categories, which is the required morphism.

The fourth isomorphism can be constructed in the same way as the second.

One might expect that the $\varliminf_{\lambda^{\prime}}$ in the proposition may be replaced by $R \lim _{\lambda^{\prime}}$, to make the statement more natural. This would need to extend the results of [KS06, Chap. 15] on derivation of indcategories cited here several times to unbounded derived categories. It is not clear whether such an extension is possible or not.

The following should be a well-known fact on the commutation of the derived pushforward and direct/derived inverse limits. We note it here since we need to treat continuous maps of sites without exact pullbacks and non-coherent sites, which do not frequently appear in the literature.

Proposition (2.2.4). 
(a) Let $S$ be a site defined by finite coverings. Let $\mathcal{C}^{\prime} \subset \operatorname{Ab}(S)$ be the full subcategory of acyclic sheaves. Then $\mathcal{C}^{\prime}$ satisfies the conditions of (2.2.1) (b).

(b) Let $f: S_{2} \rightarrow S_{1}$ be a continuous map of sites. Assume that both $S_{1}$ and $S_{2}$ are defined by finite coverings. Assume also that $f_{*}$ sends acyclic sheaves to acyclic sheaves. Then $R f_{*}$ commutes with $\varliminf_{\longrightarrow}$ and $R \biguplus$ on bounded below derived categories. That is, the diagrams

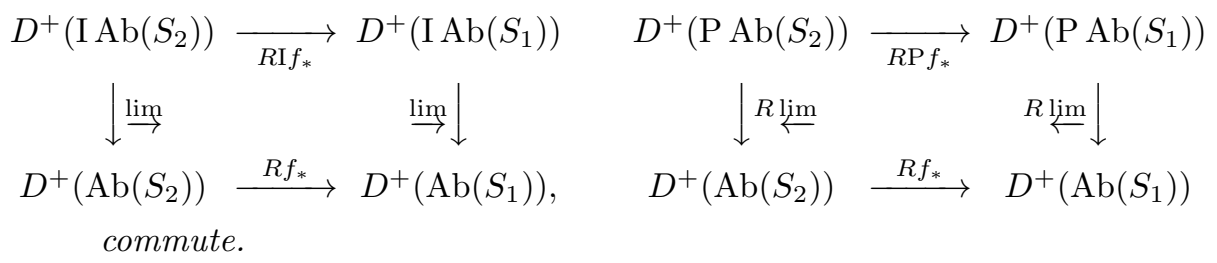

Proof. (国) The category $\mathcal{C}^{\prime}$ is closed by products and filtered direct limits by the comments in the proof of (2.1.2) (国). All the other conditions are immediate.

(b) Let $\mathcal{C}_{i}^{\prime} \subset \mathrm{Ab}\left(S_{i}\right)$ be the full subcategory of acyclic sheaves, $i=1,2$. The commutative diagrams

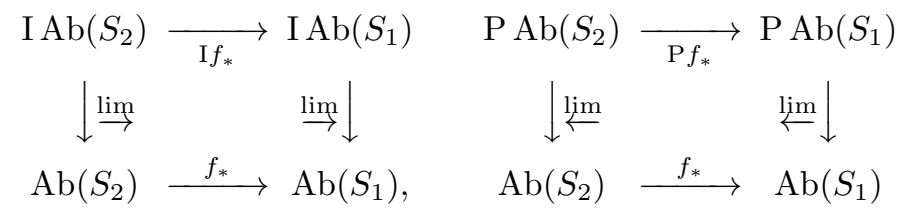

restricts to their subcategories

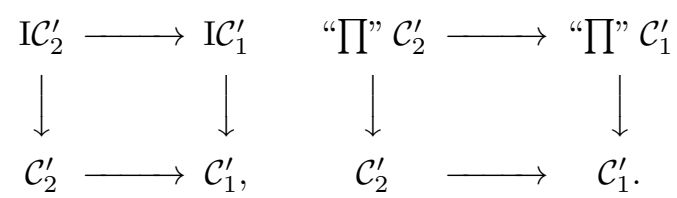

Hence the diagrams derive to commutative diagrams of the derived categories by (2.2.1).

2.3. The derived categories of ind-proalgebraic groups and of sheaves. We apply the results of the previous subsection to $\mathrm{Alg} / k$ and $\mathrm{Ab}\left(k_{\text {proet }}^{\text {indrat }}\right)$. We continue using the notation in the previous subsection about filtered direct/derived inverse limits in derived categories of ind/pro-objects. We use, however, the usual lim and lim instead of "lim" and "lim" for simplicity when they are considered in $\overrightarrow{\mathrm{IAl}} \mathrm{l} / k$, $\overleftarrow{\mathrm{PA}} \lg / k$ or IPAlg/k$k$. The $\underset{\mathrm{lim}}{\longrightarrow}$ and $\underset{\lim }{\longleftarrow}$ for sheaves mean limits as sheaves, not as ind-sheaves or pro-sheaves.

We will use the four operations formalism (about pushforward, pullback, sheafHom and tensor products) for derived categories of sites throughout the paper. A good reference (for arbitrary morphisms of sites and unbounded derived categories) is KS06, Chap. 18].

The following theorem is a summary of the site-theoretic results proved in Suz13. We clarify below which results of [Suz13] imply each statement.

Theorem (2.3.1). Let

$$
\text { Spec } k_{\text {profppf }}^{\text {perf }} \stackrel{f}{\rightarrow} \text { Spec } k_{\text {et }}^{\text {perf }} \stackrel{g}{\rightarrow} \text { Spec } k_{\text {et }}^{\text {indrat }}
$$


be the continuous maps defined by the identity and $S$ either one of these sites. Let $A=\lim _{\lambda} A_{\lambda} \in \mathrm{PAlg} / k$ with $A_{\lambda} \in \mathrm{Alg} / k$.

(a) If $B \in \mathrm{Ab}\left(k_{\text {profppf }}^{\text {perf }}\right)$, then

$$
\begin{aligned}
g_{*} R f_{*} R \mathbf{H o m}_{k_{\mathrm{profpp}}^{\text {perf }}}(A, B) & =g_{*} R \mathbf{H o m}_{k_{\mathrm{et}}^{\text {perf }}}\left(A, R f_{*} B\right) \\
& =R \mathbf{H o m}_{k_{\mathrm{et}}^{\text {indrat }}}\left(A, g_{*} R f_{*} B\right) .
\end{aligned}
$$

(Note that $g_{*}$ is an exact functor by (2.1.2) (ㄷ).)

(b) If $B=\lim _{\mu} B_{\mu}$ with $B_{\mu} \in \mathrm{Ab}(S)$, then

$$
R \operatorname{Hom}_{S}(A, B)=\underset{\mu}{\lim } R \operatorname{Hom}_{S}\left(A, B_{\mu}\right)
$$

for $S=\operatorname{Spec} k_{\text {profppf }}^{\text {perf }}$ or $\operatorname{Spec} k_{\text {et }}^{\text {perf }}$.

(c) If $B \in \mathrm{LAlg} / k$, then $R f_{*} B=B$ (and $g_{*} B=B$ as notation), and we have

$$
R \operatorname{Hom}_{S}(A, B)=\underset{\lambda}{\lim } R \operatorname{Hom}_{S}\left(A_{\lambda}, B\right)
$$

(with $S=\operatorname{Spec} k_{\mathrm{et}}^{\text {indrat }}$ allowed). Its $R \Gamma\left(k^{\prime}, \cdot\right)$ for $k^{\prime}=\bigcup k_{\nu}^{\prime} \in k^{\text {indrat }}$ with $k_{\nu}^{\prime} \in k^{\text {rat }}$ is

$$
\underset{\lambda, \nu}{\lim _{\lambda}} R \operatorname{Hom}_{S / k_{\nu}^{\prime}}\left(A_{\lambda}, B\right),
$$

where $S / k_{\nu}^{\prime}$ is the localization of $S$ at $k_{\nu}^{\prime}$ (see the notation section of $\$ 1.3$ ). If $k^{\prime}$ is a field and $S=\operatorname{Spec} k_{\mathrm{et}}^{\text {indrat }}$, then this is further isomorphic to

$$
R \operatorname{Hom}_{k_{\text {et }}^{\text {indrat }}}\left(A_{\lambda}, B\right) .
$$

(d) If $B \in \mathrm{Et} / k(\subset \mathrm{LAlg} / k)$ is uniquely divisible, then

$$
R \operatorname{Hom}_{k_{\mathrm{et}}^{\text {indrat }}}(A, B)=0 .
$$

(e) If $B \in \mathrm{Alg} / k$, then

$$
R \operatorname{Hom}_{S}(A, B)=R \operatorname{Hom}_{\mathrm{PAlg} / k}(A, B) .
$$

The corresponding results in Suz13 were proved for affine $A$. (The symbol $\mathrm{Alg} / k$ in [Suz13] denoted the category of affine quasi-algebraic groups.) The same proof works for abelian varieties as remarked after [Suz13, Thm. 2.1.5]. It even works for arbitrary proalgebraic groups; see Appendix $\mathrm{A}$.

Proof. (国) Note that $f^{*}$ is the pro-fppf sheafification functor and hence $f^{*} A=A$. ( $A$ is a sheaf even for the fpqc topology by the fpqc descent and the fact that inverses limits of sheaves are sheaves.) Therefore the sheafified adjunction between $f^{*}$ and $R f_{*}$ [KS06, Thm. 18.6.9 (iii)] gives an isomorphism

$$
R f_{*} R \mathbf{H o m}_{k_{\text {profppf }}^{\text {perf }}}(A, B)=R \mathbf{H o m}_{k_{\text {et }}^{\text {perf }}}\left(A, R f_{*} B\right)
$$

in $D\left(k_{\text {et }}^{\text {perf }}\right)$. This proves the first isomorphism. For the second, if $R f_{*} B \stackrel{\sim}{\rightarrow} J$ is an injective resolution over $\operatorname{Spec} k_{\text {et }}^{\text {perf }}$, then we have natural isomorphisms and the 
functoriality morphism of $g_{*}$ :

$$
\begin{aligned}
g_{*} R \mathbf{H o m}_{k_{\mathrm{et}}^{\text {perf }}}\left(A, R f_{*} B\right) & =g_{*} \operatorname{Hom}_{k_{\mathrm{et}}^{\text {perf }}}(A, J) \\
& \rightarrow \operatorname{Hom}_{k_{\mathrm{et}}^{\text {indrat }}}\left(g_{*} A, g_{*} J\right) \\
& \rightarrow R \mathbf{H o m}_{k_{\mathrm{et}}^{\text {indrat }}}\left(g_{*} A, g_{*} J\right) \\
& =R \operatorname{Hom}_{k_{\mathrm{et}}^{\text {indrat }}}\left(A, g_{*} R f_{*} B\right)
\end{aligned}
$$

in $D\left(k_{\mathrm{et}}^{\text {indrat }}\right)$, where the middle Hom's are the total complexes of the sheaf-Hom double complexes and $g_{*} A=A$ as notation. Applying $R \Gamma\left(k_{\mathrm{et}}^{\prime}, \cdot\right)$ for any $k^{\prime} \in$ $k^{\text {indrat }}$ and using the Leray spectral sequence for $g_{*}$, we have a morphism

$$
R \operatorname{Hom}_{k_{\mathrm{et}}^{\prime \text { perf }}}\left(A, R f_{*} B\right) \rightarrow R \operatorname{Hom}_{k_{\mathrm{et}}^{\text {indrat }} / k^{\prime}}\left(A, g_{*} R f_{*} B\right)
$$

in $D(\mathrm{Ab})$. This is an isomorphism by [Suz13, Prop. 3.7.4]. Therefore we obtain the second isomorphism.

(b) Let $S=\operatorname{Spec} k_{\mathrm{et}}^{\mathrm{perf}}$. We have a morphism

$$
\underset{\mu}{\lim } R \mathbf{H o m}_{k_{\mathrm{et}}^{\text {perf }}}\left(A, B_{\mu}\right) \rightarrow R \mathbf{H o m}_{k_{\mathrm{et}}^{\text {perf }}}(A, B)
$$

by (2.2.3). (The construction of this morphism given in the proof does not involve the subcategory $\mathcal{C}^{\prime}$.) To see this is an isomorphism, we apply $R \Gamma\left(R_{\text {et }}, \cdot\right)$ for any $R \in k^{\text {perf }}$ and take cohomology in degree $n \geq 0$ :

$$
\underset{\mu}{\lim } \operatorname{Ext}_{R_{\mathrm{et}}^{\text {perf }}}^{n}\left(A, B_{\mu}\right) \rightarrow \operatorname{Ext}_{R_{\mathrm{et}}^{\text {perf }}}^{n}(A, B) .
$$

This is an isomorphism by Suz13, Lem. 3.8.2 (2)]. Hence the result follows.

The same proof (including the proof of [Suz13, loc. cit.]) works for Spec $k_{\text {profppf }}^{\text {perf }}$ in the same manner.

(C) We have a morphism

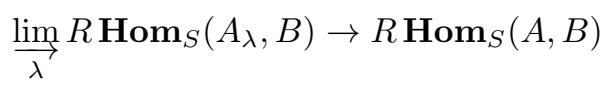

by (2.2.3) (with the same remark about $\mathcal{C}^{\prime}$ as above). Again, by taking cohomology at each object of $S$ and cohomology at each degree, we are reduced to showing that the following four induced morphisms are isomorphisms:

$$
\begin{gathered}
\underset{\lambda, \nu}{\lim } \operatorname{Ext}_{\left(R_{\nu}\right)_{\text {et }}^{\text {perf }}}^{n}\left(A_{\lambda}, B\right) \rightarrow \operatorname{Ext}_{R_{\text {et }}^{\text {perf }}}^{n}(A, B), \\
\underset{\lambda, \nu}{\lim } \operatorname{Ext}_{\left(R_{\nu}\right)_{\text {profpp }}^{\text {perf }}}^{n}\left(A_{\lambda}, B\right) \rightarrow \operatorname{Ext}_{R_{\text {profppf }}^{\text {perf }}}^{n}(A, B)
\end{gathered}
$$

for a filtered direct system $\left\{R_{\nu}\right\}$ in $k^{\text {perf }}$ with limit $R$,

$$
\underset{\lambda, \nu}{\lim } \operatorname{Ext}_{\left(k_{\nu}^{\prime}\right)_{\mathrm{et}}^{\text {indrat }}}^{n}\left(A_{\lambda}, B\right) \rightarrow \operatorname{Ext}_{k_{\mathrm{et}}^{\text {indrat }} / k^{\prime}}^{n}(A, B)
$$

for a filtered direct system $\left\{k_{\nu}^{\prime}\right\}$ in $k^{\text {rat }}$ with limit $k^{\prime}$, and

$$
\operatorname{Ext}_{k_{\mathrm{et}}^{\text {indrat }} / k^{\prime}}^{n}(A, B) \rightarrow \operatorname{Ext}_{k_{\mathrm{et}}^{\text {'indrat }}}^{n}(A, B)
$$

for a field $k^{\prime} \in k^{\text {indrat }}$. The first isomorphism is [Suz13, Lem. 3.8.2 (1)]. This lemma also works for the pro-fppf topology, so we get the second isomorphism. Since $R f_{*} B=B$ by (2.1.2) (g), [Suz13, Prop. 3.7.4] implies that

$$
\operatorname{Ext}_{k_{\text {profppf }}^{\prime \text { perf }}}^{n}(A, B)=\operatorname{Ext}_{k_{\mathrm{et}}^{\text {perf }}}^{n}(A, B)=\operatorname{Ext}_{k_{\mathrm{et}}^{\text {indrat }} / k^{\prime}}^{n}(A, B)
$$


for any $k^{\prime} \in k^{\text {indrat }}$. Hence the third isomorphism follows. The fourth isomorphism is Suz13, Thm. 2.1.5].

(d) Such a group $B$ can be written as a filtered direct limit of étale groups over $k$ whose groups of geometric points are finite-dimensional $\mathbb{Q}$-vector spaces. Hence by (b), we may assume that $B$ is an étale group over $k$ with $\operatorname{dim}_{\mathbb{Q}} B(\bar{k})<$ $\infty$. Let $k_{1}$ be a finite Galois extension of $k$ with Galois group $G$ such that $B$ becomes a constant group over $k_{1}$. Let $k^{\prime} \in k^{\text {indrat }}$ and $k_{1}^{\prime}=k^{\prime} \otimes_{k} k_{1}$. The Hochschild-Serre spectral sequence for the coefficients in $R \operatorname{Hom}_{\left(k_{1}\right)_{\mathrm{et}}^{\text {indrat }}}(A, B)=$ $R \mathbf{H o m}_{k_{\mathrm{et}}^{\text {indrat }} / k_{1}}(A, B)$ yields

$$
R \Gamma\left(G, R \operatorname{Hom}_{k_{\mathrm{et}}^{\text {indrat }} / k_{1}^{\prime}}(A, B)\right)=R \operatorname{Hom}_{k_{\mathrm{et}}^{\text {indrat }} / k^{\prime}}(A, B) .
$$

Therefore we may assume that $B$ is constant and, moreover, $B=\mathbb{Q}$. The statement to prove is thus

$$
\operatorname{Ext}_{k_{\mathrm{et}}^{\text {indrat }} / k^{\prime}}^{n}(A, \mathbb{Q})=0
$$

for $k^{\prime} \in k^{\text {indrat }}$ and $n \geq 0$. This is [Suz13, Thm. 2.1.5].

(远) By [Suz13, Thm. 2.1.5, Prop. 3.7.4 and Prop. 3.8.1], we have

$R \operatorname{Hom}_{k_{\mathrm{profpp}}^{\text {perf }}}(A, B)=R \operatorname{Hom}_{k_{\mathrm{et}}^{\text {perf }}}(A, B)=R \operatorname{Hom}_{k_{\mathrm{et}}^{\text {indrat }}}(A, B)=R \operatorname{Hom}_{\mathrm{PAlg} / k}(A, B)$.

We generalize this theorem to the case where both $A$ and $B$ are ind-proalgebraic groups.

Proposition (2.3.2). (2.3.1) remains true when all the subscripts et are replaced by proet.

Proof. This is a straightforward consequence of the fact that $A \in \mathrm{PAlg} / k$ is a sheaf for the pro-fppf topology (as commented in the proof of the theorem) and (2.1.2) (g). We only explain this for (国) and (ㄷ).

(a) We need to show that the morphisms

$$
R \operatorname{Hom}_{k_{\text {profppf }}^{\text {perf }}}(A, B) \rightarrow R \operatorname{Hom}_{k_{\text {proet }}^{\text {perf }}}\left(A, R \tilde{f}_{*} B\right) \rightarrow R \operatorname{Hom}_{k_{\text {proet }}^{\text {indrat }} / k^{\prime}}\left(A, \tilde{g}_{*} R \tilde{f}_{*} B\right)
$$

are isomorphisms for $k^{\prime} \in k^{\text {indrat }}$, where

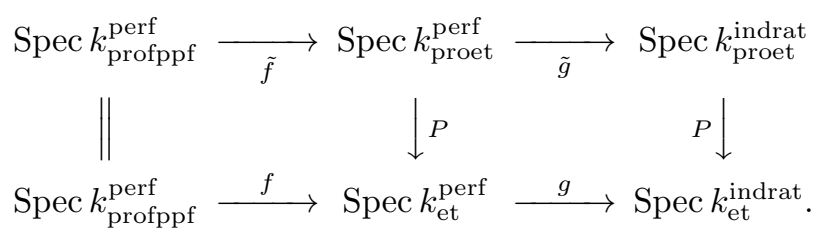

But $P^{*} A=A$. Hence the adjunction between $P^{*}$ and $R P_{*}$ reduces the statement to the original assertion (a).

(C) Similarly, we need to show that the morphism

$$
\underset{\lambda}{\lim } R \operatorname{Hom}_{k_{\text {proet }}^{\text {indrat }} / k^{\prime}}\left(A_{\lambda}, B\right) \rightarrow R \operatorname{Hom}_{k_{\text {proet }}^{\text {indrat }} / k^{\prime}}(A, B)
$$

is an isomorphism for $k^{\prime} \in k^{\text {indrat }}$. But $R P_{*} B=B$ by (2.1.2) (g). Hence again the adjunction between $P^{*}$ and $R P_{*}$ reduces the statement to the original assertion (IC). The rest can be proven similarly.

Proposition (2.3.3). Let $S$ be either Spec $k_{\text {profppf }}^{\text {perf }}$, Spec $k_{\text {proet }}^{\text {perf }}$ or Spec $k_{\text {proet }}^{\text {indrat }}$. Let $A=\underline{\lim } A_{\lambda} \in \mathrm{IPAlg} / k$ with $A_{\lambda}=\lim _{\longleftrightarrow} A_{\lambda \lambda^{\prime}} \in \mathrm{PAlg} / k$ with $A_{\lambda \lambda^{\prime}} \in \mathrm{Alg} / k$. 
(a) Let $B=\underline{\lim } B_{\mu}=\underline{\lim } \lim _{\longleftarrow} B_{\mu \mu^{\prime}} \in \mathrm{IPAlg} / k$ similarly. Then

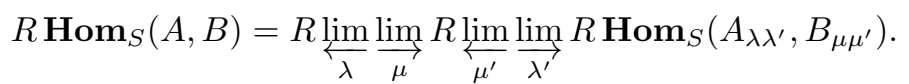

(b) Its $R \Gamma(k, \cdot)$ is

$$
\begin{aligned}
& R \operatorname{Hom}_{\mathrm{IPAlg} / k}(A, B)=R \underset{\varliminf_{\lambda}}{\lim _{\mu}} R \operatorname{Hom}_{\mathrm{PAlg} / k}\left(A_{\lambda}, B_{\mu}\right) \\
& =R \underset{\lambda}{\lim _{\lambda}} \underset{\mu}{\lim } R \underset{\mu^{\prime}}{\lim } \underset{\lambda^{\prime}}{\lim } R \operatorname{Hom}_{\mathrm{Alg} / k}\left(A_{\lambda \lambda^{\prime}}, B_{\mu \mu^{\prime}}\right) \text {. }
\end{aligned}
$$

When already $A_{\lambda}, B_{\mu} \in \mathrm{Alg} / k$ for all $\lambda, \mu$ so that $A, B \in \mathrm{IAlg} / k$, then this further equals to $R \operatorname{Hom}_{\mathrm{IAlg} / k}(A, B)$.

(c) If $B \in \mathrm{LAlg} / k$, then

$$
R \operatorname{Hom}_{S}(A, B)=R \underset{\bigsqcup_{\lambda}}{\lim _{\lambda^{\prime}}} \underset{\lim }{\longrightarrow} \operatorname{Hom}_{S}\left(A_{\lambda \lambda^{\prime}}, B\right) .
$$

(d) If $B \in \mathrm{Et} / k$ is uniquely divisible, then

$$
R \operatorname{Hom}_{k_{\text {proet }}^{\text {indrat }}}(A, B)=0 .
$$

Proof. (国) We first prove this for $S=\operatorname{Spec} k_{\text {profppf }}^{\text {perf }}$ and $\operatorname{Spec} k_{\text {proet }}^{\text {perf }}$. We will apply (2.2.3) for

$$
F=\operatorname{Hom}_{S}: \operatorname{Ab}(S)^{\mathrm{op}} \times \operatorname{Ab}(S) \rightarrow \operatorname{Ab}(S) .
$$

Let $\mathcal{C}=\mathcal{C}^{\prime}=\operatorname{Ab}(S)$. This has exact products by (2.1.2) (dd). This implies the conditions of (2.2.1) (b). Hence (2.2.3) yields morphisms and isomorphisms

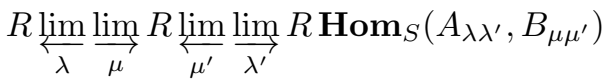

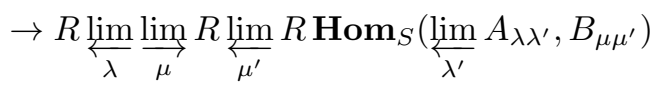

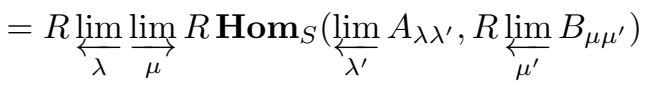

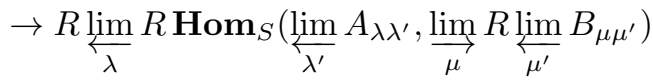

$$
\begin{aligned}
& \left.=R \operatorname{Hom}_{S} \underset{\lambda}{\left(\underset{\lambda}{\lim } \underset{\lambda^{\prime}}{\lim } A_{\lambda \lambda^{\prime}}\right.} \underset{\mu}{\lim } R \underset{\mu^{\prime}}{\lim } B_{\mu \mu^{\prime}}\right)
\end{aligned}
$$

in $D^{+}(S)$ for any

$$
\begin{aligned}
& A=\underset{\lambda}{\stackrel{\lim }{\longrightarrow}} \text { "lim" }{\overleftarrow{\lambda^{\prime}}} A_{\lambda \lambda^{\prime}} \in \operatorname{IP} \operatorname{Ab}(S), \\
& B=\underset{\mu}{\stackrel{\lim }{\longrightarrow}} \text { "lim" } B_{\mu^{\prime}}{ }_{\mu \mu^{\prime}} \in \operatorname{IP} \operatorname{Ab}(S) \text {. }
\end{aligned}
$$

Assume $A_{\lambda \lambda^{\prime}}, B_{\mu \mu^{\prime}} \in \mathrm{Alg} / k$. Then $R \lim _{\mu^{\prime}} B_{\mu \mu^{\prime}}=\lim _{\mu^{\prime}} B_{\mu \mu^{\prime}}$ by (2.1.2) (f). The first and third morphisms are isomorphisms by (2.3.1) (C) and (b), respectively.

For $S=\operatorname{Spec} k_{\text {proet }}^{\text {indrat }}$, let

$$
\text { Spec } k_{\text {profppf }}^{\text {perf }} \stackrel{f}{\rightarrow} \text { Spec } k_{\text {proet }}^{\text {perf }} \stackrel{g}{\rightarrow} \text { Spec } k_{\text {proet }}^{\text {indrat }}
$$

be the continuous maps defined by the identity. We will apply $g_{*}$ for the both sides of the isomorphism just proved for $S=\operatorname{Spec} k_{\text {proet }}^{\text {perf }}$. For the left-hand side, we have

$$
g_{*} R \mathbf{H o m}_{k_{\text {proet }}^{\text {perf }}}(A, B)=R \mathbf{H o m}_{k_{\text {proet }}^{\text {indrat }}}(A, B)
$$


by (2.3.1) (国) and (2.3.2), since $B \in \mathrm{IPAlg} / k$ and hence $R f_{*} B=B$ by (2.1.2) (g). For the right-hand side, we have

$$
\begin{aligned}
& g_{*} R \underset{\lambda}{\lim _{\lambda}} \underset{\mu}{\lim } R \underset{\mu^{\prime}}{\lim _{\lambda^{\prime}}} \underset{\lim _{\mathbf{m}}}{\operatorname{lom}} \mathbf{H o m}_{k_{\mathrm{proet}}^{\text {perf }}}\left(A_{\lambda \lambda^{\prime}}, B_{\mu \mu^{\prime}}\right) \\
& =R \underset{\lambda}{\lim _{\lambda}} \underset{\mu}{\lim } R \underset{\mu^{\prime}}{\lim _{\lambda^{\prime}}} \lim _{*} g_{*} R \mathbf{H o m}_{k_{\text {proet }}^{\text {perr }}}\left(A_{\lambda \lambda^{\prime}}, B_{\mu \mu^{\prime}}\right) \\
& =R \lim _{\lambda} \underset{\mu}{\lim } R \underset{\mu^{\prime}}{\lim } \underset{\lambda^{\prime}}{\lim } R \mathbf{H o m}_{k_{\text {proet }}^{\text {indrat }}}\left(A_{\lambda \lambda^{\prime}}, B_{\mu \mu^{\prime}}\right)
\end{aligned}
$$

by (2.2.4) and again by (2.3.1) (国).

(b). We have

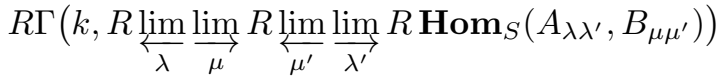

$$
\begin{aligned}
& =R \underset{\lambda}{\lim _{\mu}} \underset{\lim _{\mu}}{\lim } \underset{\lim _{\mu^{\prime}}}{\lim _{\lambda^{\prime}}} R \operatorname{Hom}_{S}\left(A_{\lambda \lambda^{\prime}}, B_{\mu \mu^{\prime}}\right) \text {. }
\end{aligned}
$$

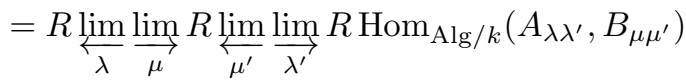

by (2.3.1) (eㅣ) and (2.3.2).

(ㄷ) The proof of (a) also applies.

(d) This follows from (c) here, (2.3.1) (d) and (2.3.2).

Proposition (2.3.4). We have fully faithful embeddings

$$
\begin{gathered}
D^{b}(\mathrm{Alg} / k) \longrightarrow D^{b}(\mathrm{PAlg} / k) \\
\downarrow \cap \\
D^{b}(\mathrm{IAlg} / k) \stackrel{\subset}{\longrightarrow} D^{b}(\mathrm{IPAlg} / k) \stackrel{\subset}{\longrightarrow} D^{b}\left(k_{\text {proet }}^{\text {indrat }}\right), \\
D^{b}(\mathrm{FGEt} / k) \subset D^{b}(\mathrm{Et} / k)=D^{b}\left(k_{\text {et }}\right) \subset D^{b}\left(k_{\text {proet }}^{\text {indrat }}\right)
\end{gathered}
$$

of triangulated categories. The same is true with $D^{b}\left(k_{\text {proet }}^{\text {indrat }}\right)$ replaced by $D^{b}\left(k_{\text {proet }}^{\text {perf }}\right)$ or $D^{b}\left(k_{\text {profppf }}^{\text {perf }}\right)$.

Proof. This follows from (2.2.2) and (2.3.3).

2.4. Serre duality and P-acyclicity. In this subsection, we mainly focus on the site Spec $k_{\text {proet }}^{\text {indrat }}$. From the next section, it is important to use $\operatorname{Spec} k_{\text {et }}^{\text {indrat }}$ and $\operatorname{Spec} k_{\text {proet }}^{\text {indrat }}$ in order to view cohomology of local fields as sheaves over the

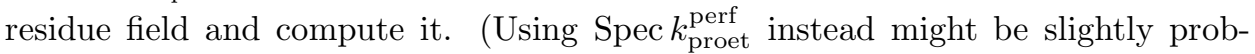
lematic for the treatment of Serre duality for semi-abelian varieties. The group $\operatorname{Ext}_{R_{\mathrm{proet}}^{\text {per }}}^{n}\left(\mathbf{G}_{m}, \mathbf{G}_{m}\right)$ for example might not be torsion for $n \geq 2$ if $R$ is not the perfection of a regular scheme and the computation done in Bre70] does not literally apply. This implies that the sheaf-Ext $\mathbf{E x t}_{k_{\text {proet }}^{\text {perf }}}^{n}\left(\mathbf{G}_{m}, \mathbf{G}_{m}\right)$ might not be torsion.

The same problem exists for Spec $k_{\text {profppf }}^{\text {perf }}$.)

For a complex $A \in D\left(k_{\text {proet }}^{\text {indrat }}\right)$, we define

$$
A^{\mathrm{SD}}=R \mathbf{H o m}_{k_{\text {proet }}^{\text {indrat }}}(A, \mathbb{Z}) .
$$


We call $A^{\mathrm{SD}}$ the Serre dual of $A$ 过 It defines a contravariant triangulated endofunctor SD on $D\left(k_{\text {proet }}^{\text {indrat }}\right)$. If $A \in \mathrm{IPAlg} / k$, then

$$
A^{\mathrm{SD}}=R \mathbf{H o m}_{k_{\mathrm{proet}}^{\text {indrat }}}(A, \mathbb{Q} / \mathbb{Z})[-1]
$$

by (2.3.3) (d). Note that this is not true for $\mathbb{Z}$, whose Serre dual is itself. For any $A \in D\left(k_{\text {proet }}^{\text {indrat }}\right)$, we have a natural morphism $A \rightarrow\left(A^{\mathrm{SD}}\right)^{\mathrm{SD}}=A^{\mathrm{SDSD}}$. If this is an isomorphism, we say that $A$ is Serre reflexive. Serre reflexive complexes form a full triangulated subcategory of $D\left(k_{\text {proet }}^{\text {indrat }}\right)$, where the functor SD is a contravariant auto-equivalence with inverse itself.

\section{Proposition (2.4.1).}

(a) If $A \in \mathrm{PAlg} / k$, then

$$
\mathbf{E x t}_{k_{\text {proet }}^{\text {indrat }}}^{n}(A, \mathbb{Q} / \mathbb{Z})=0
$$

for $n \neq 0,1$. We have

$$
\operatorname{Hom}_{k_{\text {proet }}^{\text {indrat }}}(A, \mathbb{Q} / \mathbb{Z})=\operatorname{Hom}_{k_{\text {proet }}^{\text {indrat }}}\left(\pi_{0}(A), \mathbb{Q} / \mathbb{Z}\right) .
$$

(b) The objects of the categories

$$
D^{b}\left(\mathrm{PAlg}_{\mathrm{uc}} / k\right), D^{b}\left(\operatorname{IAlg}_{\mathrm{uc}} / k\right), D^{b}(\mathrm{FGEt} / k)
$$

are Serre reflexive. The functor $\mathrm{SD}$ gives an auto-equivalence on $D^{b}\left(\operatorname{Alg}_{\mathrm{uc}} / k\right)$, which agrees with the Breen-Serre duality on perfect unipotent groups ([Ser60, 8.4, Remarque], Mil06, III, Thm. 0.14]) shifted by -1.5 It also gives an auto-equivalence on $D^{b}(\mathrm{FEt} / k)$, which agrees with the Pontryagin duality $\mathrm{PD}=\mathbf{H o m}_{k}(\cdot, \mathbb{Q} / \mathbb{Z})$ shifted by -1 . It further gives an auto-equivalence on $D^{b}(\mathrm{FGEt} / k)$, and an equivalence between $D^{b}\left(\mathrm{PAlg}_{\mathrm{uc}} / k\right)$ and $D^{b}\left(\mathrm{IAlg}_{\mathrm{uc}} / k\right)$. For $A \in \mathrm{PAlg} / k$ (resp. $A \in \mathrm{IAlg} / k$ ), we have: $H^{1} A^{\mathrm{SD}}=\pi_{0}(A)^{\mathrm{PD}}$;

$$
H^{2} A^{\mathrm{SD}}=\mathbf{E x t}_{k_{\mathrm{proet}}^{\text {indrat }}}^{1}(A, \mathbb{Q} / \mathbb{Z})
$$

is a connected ind-unipotent (resp. pro-unipotent) group; and $H^{n} A^{\mathrm{SD}}=0$ for $n \neq 1,2$.

(c) Let $A$ be (the perfection of) a semi-abelian variety over $k$. Let TA be the Tate module of $A$, i.e. the pro-finite-étale group over $k$ given by the inverse limit of the kernel of multiplication by $n \geq 1$ on $A \mid \bar{G}$ Let $\bar{A}$ be the proétale universal covering group of $A$, i.e. the inverse limit of multiplication by $n \geq 1$ on $A$. Then the morphism $A \rightarrow T A[1]$ (where [1] is the shift) coming from the natural exact sequence

$$
0 \rightarrow T A \rightarrow \bar{A} \rightarrow A \rightarrow 0
$$

\footnotetext{
4 Milne Mil06 III, 0] used the term "Breen-Serre duality". Breen's work Bre78 brought Serre's observation Ser60 8.4, Remarque] to the setting of the perfect étale site and provided Artin-Milne AM76 a basis of their "second context" of global duality. Just to make it notationally parallel to and easily distinguishable with Cartier duality CD and Pontryagin duality PD, we omit Breen's name and use the symbol SD in this paper. Of course our treatment of the functor SD heavily relies on the work of both Breen and Serre.

${ }^{5}$ Strictly speaking, the objects of $\mathrm{Alg}_{\mathrm{uc}} / k$ are not exactly unipotent since their component groups can be any objects of FEt/ $k$ not necessarily $p$-primary. The Breen-Serre duality for finite étale $p$-primary groups agrees with the Pontryagin duality [Mil06, III, Lem. 0.13 (b)].

6 This is a reduced scheme due to the perfection. A perfect scheme is reduced. Hence $T A$ is the Pontryagin dual of the torsion part of the discrete Galois module $A(\bar{k})$.
} 
in $\mathrm{Ab}\left(k_{\text {proet }}^{\text {indrat }}\right)$ and (b) gives isomorphisms

$$
A^{\mathrm{SDSD}} \stackrel{\sim}{\rightarrow} T A[1]^{\mathrm{SDSD}}=T A[1] .
$$

We also have $A^{\mathrm{SD}}=(T A)^{\mathrm{PD}}[-2]$.

(d) For any $A \in \mathrm{PAlg} / k$, there is a canonical morphism from $A^{\mathrm{SDSD}}$ to the inverse limit $\lim _{n} A$ of multiplication by $n \geq 1$ placed in degree -1 , so that we have

$$
A^{\mathrm{SDSD}}=\left[\stackrel{\lim }{n}_{n} A \rightarrow A\right]
$$

where the morphism $\lim _{n} A \rightarrow A$ is the projection to the $n=1$ term and [·] denotes the mapping cone. To give a more explicit description, let $A_{0}$ be the identity component of $A, A_{\mathrm{u}}$ the maximal pro-unipotent subgroup of $A_{0}, A_{\mathrm{sAb}}=A_{0} / A_{\mathrm{u}}$ the maximal semi-abelian quotient of $A_{0}$. Then we have $\lim _{n} A=\overline{A_{\mathrm{sAb}}}$, whose Serre dual is zero. The commutative diagram with exact rows

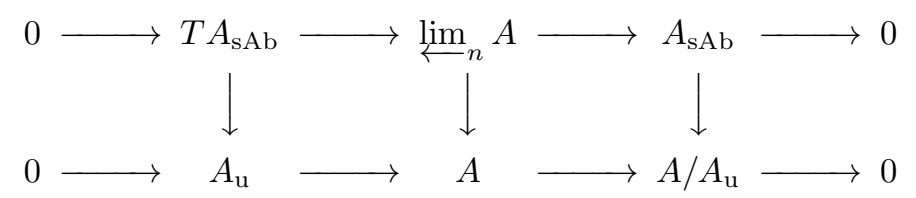

gives a distinguished triangle7

$$
\left[T A_{\mathrm{sAb}} \rightarrow A_{\mathrm{u}}\right] \rightarrow\left[\varliminf_{n} A \rightarrow A\right] \rightarrow \pi_{0}(A) .
$$

In particular, we have $A^{\mathrm{SDSD}} \in D^{b}\left(\mathrm{PAlg}_{\mathrm{uc}} / k\right)$. We also have $A^{\mathrm{SD}} \in$ $D^{b}\left(\operatorname{IAlg}_{\mathrm{uc}} / k\right)$, which is Serre reflexive.

Proof. (a) For any $n$, the sheaf in question is the pro-étale sheafification of the presheaf

$$
k^{\prime} \in k^{\text {indrat }} \mapsto \operatorname{Ext}_{k_{\text {proet }}^{\text {ind rat }} / k^{\prime}}^{n}(A, \mathbb{Q} / \mathbb{Z}) .
$$

Let $k^{\prime}=\bigcup k_{\nu}^{\prime} \in k^{\text {indrat }}$ with $k_{\nu}^{\prime} \in k^{\text {rat }}$. Then the group on the right can also be written as

$$
\operatorname{Ext}_{k_{\text {profppf }}^{\text {perf }} / k^{\prime}}^{n}(A, \mathbb{Q} / \mathbb{Z})=\operatorname{Ext}_{k_{\text {et }}^{\text {indrat }} / k^{\prime}}^{n}(A, \mathbb{Q} / \mathbb{Z})=\underset{\nu}{\lim } \operatorname{Ext}_{\left(k_{\nu}^{\prime}\right)_{\text {et }}^{\text {indrat }}}^{n}(A, \mathbb{Q} / \mathbb{Z})
$$

by (2.3.1) (a) (ㄷ) and (2.3.2).

For the statement for $n \neq 0,1$, it is enough, replacing $k_{\nu}^{\prime}$ with $k$, to show that the étale sheafification of

$$
k^{\prime} \in k_{\mathrm{et}} \mapsto \operatorname{Ext}_{k_{\mathrm{et}}^{\text {indrat }}}^{n}(A, \mathbb{Q} / \mathbb{Z})
$$

is zero (for any perfect field $k$ ), where $k_{\text {et }}$ is the category of étale $k$-algebras. Equivalently, it is enough to show that the group

$$
\operatorname{Ext}_{\bar{k}_{\text {et }}^{\text {indrat }}}^{n}(A, \mathbb{Q} / \mathbb{Z})=\operatorname{Ext}_{k_{\mathrm{et}}^{\text {indrat }} / \bar{k}}^{n}(A, \mathbb{Q} / \mathbb{Z})=\underset{k^{\prime} / k \text { finite }}{\lim _{k_{\mathrm{et}}}} \operatorname{Ext}_{k_{\text {ind }}^{\text {ind }}}^{n}(A, \mathbb{Q} / \mathbb{Z})
$$

\footnotetext{
7 Perhaps a more helpful description of $A^{\mathrm{SDSD}}=[\lim A \rightarrow A]$ is that its $H^{0}$ is the quotient of $A$ by the maximal semiabelian subgroup $A_{\mathrm{sAb}}^{\prime}$ of $A \overleftarrow{\text { (so }} H^{0}$ is the maximal quotient of $A$ that belongs to $\mathrm{PAlg}(\mathrm{uc})$ and $H^{-1}$ the Tate module of $A_{\mathrm{sAb}}^{\prime}$. The composite $A_{\mathrm{sAb}}^{\prime} \hookrightarrow A_{0} \rightarrow A_{\mathrm{sAb}}$ is an isogeny.
} 
is zero for $n \neq 0,1$, where $\bar{k}$ is an algebraic closure of $k$. For this, we may assume that $k=\bar{k}$. We have

$$
\operatorname{Ext}_{k_{\mathrm{et}}^{\text {indrat }}}^{n}(A, \mathbb{Q} / \mathbb{Z})=\underset{m}{\lim _{\vec{m}}} \operatorname{Ext}_{k_{\mathrm{et}}^{\text {indrat }}}^{n}(A, \mathbb{Z} / m \mathbb{Z})=\underset{m}{\lim } \operatorname{Ext}_{\mathrm{PAlg} / k}^{n}(A, \mathbb{Z} / m \mathbb{Z})
$$

by (2.3.1) (b), (E). This final group is the Pontryagin dual of the $n$-th homotopy group of $A$ over $k$ in the sense of Serre [Ser60, $\S 5$, Cor. to Prop. 7]. It is zero for $n \neq 0,1$ by [Ser60, $\S 10$, Thm. 2].

The statement for $n=0$ is proven similarly.

(b) First assume that $A \in \operatorname{Alg}_{\mathrm{uc}} / k$. Let $g: \operatorname{Spec} k_{\text {proet }}^{\text {perf }} \rightarrow \operatorname{Spec} k_{\text {proet }}^{\text {indrat }}$ be the continuous map defined by the identity. Then

$$
A^{\mathrm{SD}}=R \operatorname{Hom}_{k_{\text {proet }}^{\text {indrat }}}(A, \mathbb{Q} / \mathbb{Z})[-1]=g_{*} R \operatorname{Hom}_{k_{\text {proet }}^{\text {perf }}}(A, \mathbb{Q} / \mathbb{Z})[-1]
$$

by (2.1.2) (国), (2.3.1) (国) and (2.3.2). Let $P: \operatorname{Spec} k_{\text {proet }}^{\text {perf }} \rightarrow \operatorname{Spec} k_{\text {et }}^{\text {perf }}$ be the morphism defined by the identity. Then $R P_{*} \mathbb{Q} / \mathbb{Z}=\mathbb{Q} / \mathbb{Z}$ by (2.1.2) (g) and $P^{*} A=$ $A$. Hence

$$
R P_{*} R \mathbf{H o m}_{k_{\text {proet }}^{\text {perf }}}(A, \mathbb{Q} / \mathbb{Z})=R \mathbf{H o m}_{k_{\mathrm{et}}^{\text {perf }}}(A, \mathbb{Q} / \mathbb{Z}) .
$$

The $p$-primary part of the right-hand side is the Breen-Serre dual of $A$ by definition [Mil06, III, Thm. 0.14], which is in $D^{b}\left(\operatorname{Alg}_{\mathrm{uc}} / k\right)$. The prime-to- $p$ part is the Pontryagin dual of the prime-to- $p$ part of $A$ (or $\pi_{0}(A)$ ). With $P^{*} R P_{*}=$ id, we have

$$
R \mathbf{H o m}_{k_{\mathrm{proet}}^{\text {perr }}}(A, \mathbb{Q} / \mathbb{Z})=P^{*} R \mathbf{H o m}_{k_{\mathrm{et}}^{\text {perf }}}(A, \mathbb{Q} / \mathbb{Z}),
$$

hence

$$
A^{\mathrm{SD}}=g_{*} P^{*} R \mathbf{H o m}_{k_{\mathrm{et}}^{\mathrm{perf}}}(A, \mathbb{Q} / \mathbb{Z})[-1] .
$$

Note that the objects in $\mathrm{Alg} / k$ are already pro-étale sheaves and hence invariant under the pro-étale sheafification $P^{*}$. Hence we know that $A^{\mathrm{SD}}$ is the Breen-Serre dual of $A$ shifted by -1 in the $p$-primary part and the Pontryagin dual of $A$ in the prime-to- $p$ part shifted by -1 . In particular, we have by [Mil06, III, Lem. 0.13 (b), (c), (d)]: $H^{1} A^{\mathrm{SD}}=\pi_{0}(A)^{\mathrm{PD}} ; H^{2} A^{\mathrm{SD}} \in \mathrm{Alg} / k$ is connected unipotent; and $H^{n}=0$ for $n \neq 1,2$.

We show that $A^{\mathrm{SDSD}}=A$. The Breen-Serre duality [Mil06, III, Thm. 0.14] and the Pontryagin duality show that

$$
A=R \mathbf{H o m}_{k_{\mathrm{et}}^{\text {perf }}}\left(R \mathbf{H o m}_{k_{\mathrm{et}}^{\text {perf }}}(A, \mathbb{Q} / \mathbb{Z}), \mathbb{Q} / \mathbb{Z}\right) .
$$

By $R P_{*} \mathbb{Q} / \mathbb{Z}=\mathbb{Q} / \mathbb{Z}, P^{*} R P_{*}=$ id and $P^{*} A=A$, we have

$$
A=R \mathbf{H o m}_{k_{\text {proet }}^{\text {perf }}}\left(P^{*} R \mathbf{H o m}_{k_{\mathrm{et}}^{\text {perf }}}(A, \mathbb{Q} / \mathbb{Z}), \mathbb{Q} / \mathbb{Z}\right) .
$$

Applying $g_{*}$, we have a morphism

$$
\begin{aligned}
A & =g_{*} R \mathbf{H o m}_{k_{\text {proet }}^{\text {perf }}}\left(P^{*} R \mathbf{H o m}_{k_{\mathrm{et}}^{\text {perf }}}(A, \mathbb{Q} / \mathbb{Z}), \mathbb{Q} / \mathbb{Z}\right) \\
& \rightarrow R \mathbf{H o m}_{k_{\text {proet }}^{\text {indrat }}}\left(g_{*} P^{*} R \mathbf{H o m}_{k_{\mathrm{et}}^{\text {perf }}}(A, \mathbb{Q} / \mathbb{Z}), \mathbb{Q} / \mathbb{Z}\right) \\
& =A^{\mathrm{SDSD}} .
\end{aligned}
$$

Since $R \mathbf{H o m}_{k_{\mathrm{et}}^{\text {perf }}}(A, \mathbb{Q} / \mathbb{Z}) \in D^{b}\left(\operatorname{Alg}_{\mathrm{uc}} / k\right)$, this morphism is an isomorphism by (2.3.1) (国) and (2.3.2) (applied to the cohomologies of the complex $R \mathbf{H o m}_{k_{\mathrm{et}}^{\text {perf }}}(A, \mathbb{Q} / \mathbb{Z})$ ). Thus $A^{\mathrm{SDSD}}=A$.

If $A=\lim _{\lambda} A_{\lambda} \in \mathrm{PAlg} \operatorname{uc}_{\mathrm{uc}} / k$, then $A^{\mathrm{SD}}=\lim _{\lambda} A_{\lambda}^{\mathrm{SD}}$ by (2.3.3) (国). Since the direct limit of sheaves is exact, we have $H^{n} A^{\mathrm{SD}}=\lim _{\longrightarrow} H^{n} A_{\lambda}^{\mathrm{SD}}$ for any $n$. Hence $A^{\mathrm{SD}} \in$ 
$D^{b}\left(\operatorname{IAlg}_{\mathrm{uc}} / k\right)$. On the other hand, if $A=\lim A_{\lambda} \in \operatorname{IAlg}_{\mathrm{uc}} / k$, then $A^{\mathrm{SD}}=R \lim A_{\lambda}^{\mathrm{SD}}$

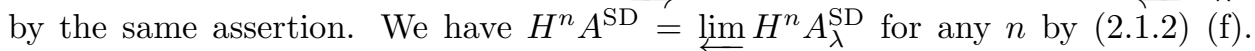
Hence $A^{\mathrm{SD}} \in D^{b}\left(\mathrm{PAlg}_{\mathrm{uc}} / k\right)$. Therefore the statements about $D^{b}\left(\mathrm{PAlg} \lg _{\mathrm{uc}} / k\right)$ and $D^{b}\left(\operatorname{IAlg}_{\mathrm{uc}} / k\right)$ are reduced to the statements about $D^{b}\left(\mathrm{Alg}_{\mathrm{uc}} / k\right)$, which have already been proved above.

The statement for $D^{b}(\mathrm{FGEt} / k)$ follows from the easy computation $\mathbb{Z}^{\mathrm{SD}}=\mathbb{Z}$.

(ㄷ) By (2.3.3) (国), we have

$$
R \mathbf{H o m}_{k_{\text {proet }}^{\text {indrat }}}(\bar{A}, \mathbb{Q} / \mathbb{Z})=\underset{n}{\lim _{\longrightarrow}} R \operatorname{Hom}_{k_{\text {proet }}^{\text {indrat }}}(\bar{A}, \mathbb{Z} / n \mathbb{Z})=0
$$

since $\bar{A} \in \mathrm{PAlg} / k$ is uniquely divisible. With the vanishing result (a) above, we have

$$
\operatorname{Ext}_{k_{\text {proet }}^{\text {indrat }}}^{1}(A, \mathbb{Q} / \mathbb{Z})=\operatorname{Hom}_{k_{\text {proet }}^{\text {indrat }}}(T A, \mathbb{Q} / \mathbb{Z})
$$

by the universal covering exact sequence. Hence

$$
A^{\mathrm{SD}}=R \mathbf{H o m}_{k_{\text {proet }}^{\text {indrat }}}(A, \mathbb{Q} / \mathbb{Z})[-1]=\mathbf{H o m}_{k_{\text {proet }}^{\text {indrat }}}(T A, \mathbb{Q} / \mathbb{Z})[-2]=(T A)^{\mathrm{SD}}[-1] .
$$

Therefore $A^{\mathrm{SDSD}}=T A[1]$.

(d) We have

$$
A^{\mathrm{SD}}=R \mathbf{H o m}_{k_{\text {proet }}^{\text {indrat }}}(A, \mathbb{Q} / \mathbb{Z})[-1]=\underset{m}{\lim } R \operatorname{Hom}_{k_{\text {proet }}^{\text {indrat }}}(A, \mathbb{Z} / m \mathbb{Z})[-1]
$$

by (2.3.3) (a), which is killed after tensoring with $\mathbb{Q}$. Hence the same assertion implies that

$$
\left(\stackrel{\lim }{n}_{n} A\right)^{\mathrm{SD}}=\underset{n}{\lim }\left(A^{\mathrm{SD}}\right)=0
$$

Therefore we have a natural morphism and an isomorphism

$$
\left[\varliminf_{n} A \rightarrow A\right] \rightarrow\left[\varliminf_{n} \varliminf_{n} A \rightarrow A\right]^{\mathrm{SDSD}}=A^{\mathrm{SDSD}} .
$$

We will know that the first morphism is an isomorphism once we show that $\left[\lim _{n} A \rightarrow\right.$ $A] \in D^{b}\left(\mathrm{PAlg}_{\mathrm{uc}} / k\right)$ (whose objects are Serre reflexive by (b) $)$.

Note that the exact endofunctor $\lim _{n}$ on PAlg/ $k$ kills protorsion groups and hence profinite groups and pro-unipotent groups. This implies that $\lim _{n} A=$ $\varliminf_{n} A_{\mathrm{sAb}}=\overline{A_{\mathrm{sAb}}}$. This yields the stated commutative diagram. In particular, the mapping cone $\left[A_{\mathrm{sAb}} \rightarrow A / A_{\mathrm{u}}\right]$ is quasi-isomorphic to the mapping cone of the morphism of complexes $\left[T A_{\mathrm{sAb}} \rightarrow A_{\mathrm{u}}\right] \rightarrow\left[\lim _{n} A \rightarrow A\right]$. This induces the mapping cone distinguished triangle

$$
\left[T A_{\mathrm{sAb}} \rightarrow A_{\mathrm{u}}\right] \rightarrow\left[\check{L}_{n} A \rightarrow A\right] \rightarrow\left[A_{\mathrm{sAb}} \rightarrow A / A_{\mathrm{u}}\right] .
$$

Since the kernel of $A_{\mathrm{sAb}} \rightarrow A / A_{\mathrm{u}}$ is zero and the cokernel is $\pi_{0}(A)$, we obtain the stated distinguished triangle. This implies that $\left[\lim _{n} A \rightarrow A\right] \in D^{b}\left(\mathrm{PAlg}_{\mathrm{uc}} / k\right)$ since $\left[T A_{\mathrm{sAb}} \rightarrow A_{\mathrm{u}}\right], \pi_{0}(A) \in D^{b}\left(\mathrm{PAlg}_{\mathrm{uc}} / k\right)$. Therefore $A^{\mathrm{SDSD}}=\left[\lim _{n} A \rightarrow A\right] \in$ $D^{b}\left(\mathrm{PAlg}_{\mathrm{uc}} / k\right)$.

Applying the Serre dual to the stated distinguished triangle and using $\left(\lim _{n} A\right)^{\mathrm{SD}}=$ 0 , we have a distinguished triangle

$$
\pi_{0}(A)^{\mathrm{SD}} \rightarrow A^{\mathrm{SD}} \rightarrow\left[T A_{\mathrm{sAb}} \rightarrow A_{\mathrm{u}}\right]^{\mathrm{SD}} .
$$

Hence $A^{\mathrm{SD}} \in D^{b}\left(\operatorname{IAlg}_{\mathrm{uc}} / k\right)$. 
As examples, we have

$$
\begin{gathered}
\left.W_{n}^{\mathrm{SD}}=W_{n}[-2] \quad \text { Mil06, III, Lem. } 0.13(\mathrm{c})\right], \\
W^{\mathrm{SD}}=\underset{n}{\lim } W_{n}^{\mathrm{SD}}=\underset{n}{\lim } W_{n}[-2], \\
(\mathbb{Z} / n \mathbb{Z})^{\mathrm{SD}}=\mathbb{Z} / n \mathbb{Z}[-1], \quad \mathbb{Z}^{\mathrm{SD}}=\mathbb{Z}, \\
\mathbf{G}_{m}^{\mathrm{SD}}=\bigoplus_{l \neq p}\left(\mathbb{Q}_{l} / \mathbb{Z}_{l}\right)(-1)[-2], \quad A^{\mathrm{SD}}=(T A)^{\mathrm{PD}}[-2],
\end{gathered}
$$

where $n \geq 1, W_{n}$ the group of Witt vectors of length $n, W=\lim W_{n},\left(\mathbb{Q}_{l} / \mathbb{Z}_{l}\right)(-1)$ the negative Tate twist and $A$ an abelian variety over $k$. (Recall that all the groups here are the perfections of the corresponding group schemes. In particular, the $p$-th power map on the quasi-algebraic group $\mathbf{G}_{m}$ is an isomorphism.)

Before introducing the notion of P-acyclicity, we discuss sheaves locally of finite presentation on Spec $k_{\text {et }}^{\text {indrat }}$. A sheaf $F \in \operatorname{Set}\left(k_{\text {et }}^{\text {indrat }}\right)$ is said to be locally of finite presentation if it commutes with filtered direct limits as a functor on $k^{\text {indrat }}$. Such a sheaf is automatically a pro-étale sheaf: $F \in \operatorname{Set}\left(k_{\text {proet }}^{\text {indrat }}\right)$. For $k^{\prime}=\bigcup k_{\lambda}^{\prime} \in k^{\text {indrat }}$ with $k_{\lambda} \in k^{\text {rat }}$, we define

$$
F^{\mathrm{fp}}\left(k^{\prime}\right)=\underset{\lambda}{\lim } F\left(k_{\lambda}^{\prime}\right) .
$$

Clearly $F^{\mathrm{fp}}$ is locally of finite presentation. We see that $F^{\mathrm{fp}} \in \operatorname{Set}\left(k_{\mathrm{et}}^{\text {indrat }}\right)$ since an étale covering $k^{\prime \prime} / k^{\prime}$ in $k^{\text {indrat }}$ is a filtered direct limit of étale coverings in $k^{\text {rat }}$. We have a natural morphism $F^{\mathrm{fp}} \rightarrow F$, which is an isomorphism if and only if $F$ is locally of finite presentation. If $\left\{F_{\lambda}\right\}$ is a filtered direct system of sheaves locally of finite presentation, then $\lim F_{\lambda}$ is locally of finite presentation. Since objects of LAlg/ $k$ and $\mathrm{Alg} / k$ in particular are locally of finite presentation, it follows that objects of IAlg/ $k$ are also locally of finite presentation. If $A \in \mathrm{PAlg} / k$ and $B \in \mathrm{LAlg} / k$, then $\mathbf{E x t}_{k_{\mathrm{et}}^{\text {indrat }}}^{n}(A, B)$ for any $n \geq 0$ is locally of finite presentation by (2.3.1) (IC). In particular, $\mathbf{E x} \mathbf{t}_{k_{\mathrm{et}}^{\text {indrat }}}^{n}(A, B)$ and $\mathbf{E x t}_{k_{\text {proet }}^{\text {indrat }}}^{n}(A, B)$ are equal as functors on $k^{\text {indrat }}$. If $f: E \rightarrow F \in \operatorname{Set}\left(k_{\mathrm{et}}^{\text {indrat }}\right)$ is a morphism between sheaves locally of finite presentation, then the property of $f$ being injective, surjective or invertible can be tested on geometric points (i.e. if $E\left(k^{\prime}\right) \hookrightarrow F\left(k^{\prime}\right)$ for any algebraically closed field $k^{\prime}$ over $k$, then $E \hookrightarrow F$ etc.). Similarly, if $A \in \operatorname{Ab}\left(k_{\text {et }}^{\text {indrat }}\right)$ is locally of finite presentation with vanishing geometric points (i.e. $A\left(k^{\prime}\right)=0$ for any algebraically closed field $k^{\prime}$ over $k$ ), then $A=0$. This argument has already been used in the proof of (2.4.1) (国). A sheaf $F \in \operatorname{Set}\left(k_{\mathrm{et}}^{\text {indrat }}\right)$ is representable by an étale scheme over $k$ if and only if it is locally of finite presentation and $F\left(k^{\prime}\right)$ does not depend on algebraically closed $k^{\prime}$ over $k$, in which case $F=F(\bar{k})$ as $\operatorname{Gal}(\bar{k} / k)$-sets.

Now let $P:$ Spec $k_{\text {proet }}^{\text {indrat }} \rightarrow$ Spec $k_{\mathrm{et}}^{\text {indrat }}$ be the morphism defined by the identity. We say that a sheaf $A \in \mathrm{Ab}\left(k_{\mathrm{et}}^{\text {indrat }}\right)$ is $P$-acyclic if the natural morphism

$$
A \rightarrow R P_{*} P^{*} A
$$


is an isomorphism 8 This is equivalent that $A \in \mathrm{Ab}\left(k_{\text {proet }}^{\text {indrat }}\right)$ and $H^{n}\left(k_{\text {et }}^{\prime}, A\right) \stackrel{\sim}{\rightarrow}$ $H^{n}\left(k_{\text {proet }}^{\prime}, A\right)$ for all $k^{\prime} \in k^{\text {indrat }}$. We say that a complex $A \in D^{+}\left(k_{\mathrm{et}}^{\text {indrat }}\right)$ is $P$ acyclic if its cohomology is $\mathrm{P}$-acyclic in each degree. This implies that

$$
A \stackrel{\sim}{\rightarrow} R P_{*} P^{*} A \quad \text { in } \quad D^{+}\left(k_{\mathrm{et}}^{\text {indrat }}\right)
$$

by writing down the hypercohomology spectral sequence for $R P_{*} P^{*} A$. If $0 \rightarrow A \rightarrow$ $B \rightarrow C \rightarrow 0$ is an exact sequence in $\mathrm{Ab}\left(k_{\mathrm{et}}^{\text {indrat }}\right)$ and any two of the terms are $\mathrm{P}$ acyclic, then so is the other. P-acyclicity of a sheaf is preserved under filtered direct limits. If $f:$ Spec $k^{\prime} \rightarrow$ Spec $k$ is a finite étale morphism and $A^{\prime} \in \operatorname{Ab}\left(k_{\text {et }}^{\text {indrat }}\right)$ is $\mathrm{P}$-acyclic, then the Weil restriction $f_{*} A^{\prime} \in \mathrm{Ab}\left(k_{\mathrm{et}}^{\text {indrat }}\right)$ is $\mathrm{P}$-acyclic. When $A \in$ $D^{+}\left(k_{\text {et }}^{\text {indrat }}\right)$ is $\mathrm{P}$-acyclic, we will use the same letter $A$ to mean its inverse image $P^{*} A \in D^{+}\left(k_{\text {proet }}^{\text {indrat }}\right)$. Here is a criterion of P-acyclicity.

\section{Proposition (2.4.2).}

(a) If $A \in \mathrm{LAlg} / k$ or IAlg $/ k$, then $A$ is P-acyclic. More generally, any sheaf $A \in \mathrm{Ab}\left(k_{\mathrm{et}}^{\text {indrat }}\right)$ locally of finite presentation is $P$-acyclic.

(b) If $A \in \mathrm{PAlg} / k$ can be written as $A=\varliminf_{n>1} A_{n}$ with $A_{n} \in \mathrm{Alg} / k$ such that all the transition morphisms $\varphi_{n}: A_{n+1} \rightarrow A_{n}$ are surjective with connected unipotent kernel, then $A$ is P-acyclic.

Proof. (国) We already saw above that objects of LAlg $/ k$ and IAlg $/ k$ are locally of finite presentation and a sheaf locally of finite presentation is in $\mathrm{Ab}\left(k_{\text {proet }}^{\text {indrat }}\right)$. Let $A \in \mathrm{Ab}\left(k_{\text {et }}^{\text {indrat }}\right)$ be locally of finite presentation (so $A \in \mathrm{Ab}\left(k_{\text {proet }}^{\text {indrat }}\right)$ ). We need to show that $H^{n}\left(k_{\text {proet }}^{\prime}, A\right)=H^{n}\left(k_{\text {et }}^{\prime}, P_{*} A\right)$ for $n \geq 1$ and $k^{\prime} \in k^{\text {indrat }}$. In Suz13, Prop. 3.3.1], a statement corresponding to pro-fppf and fppf cohomology instead of pro-étale and étale cohomology is proved. The same proof works.

(b) Let $B_{n}$ be the maximal connected unipotent subgroup of $A_{n}$. Then $C:=$ $A_{n} / B_{n} \in \operatorname{Alg} / k$ does not depend on $n$ by assumption and $B=\lim B_{n}$ satisfies the

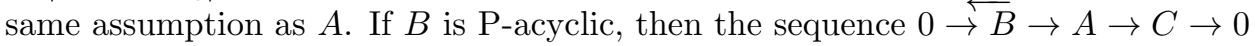
is exact in $\mathrm{Ab}\left(k_{\mathrm{et}}^{\mathrm{indrat}}\right)$, and $A$ is $\mathrm{P}$-acyclic. Therefore we may assume that all $A_{n}$ are connected unipotent. Then $H^{m}\left(k_{\text {et }}^{\prime}, A_{n}\right)=0$ for all $m \geq 1$. Therefore we have $R^{m} \prod_{n} A_{n}=0$ for all $m \geq 1$ by [Roo06, Prop. 1.6]. Therefore by [Roo06, Thm. 2.1, Lem. 2.2 and Rmk. 2.3], the object $R \varliminf A_{n} \in D\left(k_{\text {et }}^{\text {indrat }}\right)$ is represented by the complex

$$
\prod_{n} A_{n} \rightarrow \prod_{n} A_{n}
$$

concentrated in degrees 0 and 1 , where the morphism is given by $\left(a_{n}\right) \mapsto\left(a_{n}-\right.$ $\left.\varphi_{n}\left(a_{n+1}\right)\right)$. Note that the morphisms $\varphi_{n}: A_{n+1}\left(k^{\prime}\right) \rightarrow A_{n}\left(k^{\prime}\right)$ is surjective for all $k^{\prime} \in k^{\text {indrat }}$ and $n$ since $\operatorname{ker}\left(\varphi_{n}\right)$ is connected unipotent. Therefore the evaluated complex

$$
\prod_{n} A_{n}\left(k^{\prime}\right) \rightarrow \prod_{n} A_{n}\left(k^{\prime}\right)
$$

which is $R \lim A_{n}\left(k^{\prime}\right) \in D(\mathrm{Ab})$, is concentrated in degree 0 . Hence $R \lim A_{n}=A$ in $D\left(k_{\mathrm{et}}^{\text {indrat }}\right)$. Therefore

$$
R \Gamma\left(k_{\mathrm{et}}^{\prime}, A\right)=R \Gamma\left(k_{\mathrm{et}}^{\prime}, R \lim _{\longleftarrow} A_{n}\right)=R \varliminf_{\longleftarrow} R \Gamma\left(k_{\mathrm{et}}^{\prime}, A_{n}\right)=R \lim _{\longleftarrow} A_{n}\left(k^{\prime}\right)=A\left(k^{\prime}\right) .
$$

\footnotetext{
8 The "P" in "P-acyclicity" is just the initial letter of "pro" and not (necessarily) a variable. We just avoid naming it as "pro-acyclicity", since it is not a pro-object version of some acyclicity property.
} 
Since $R \lim _{n} A_{n}=A$ in $D\left(k_{\text {proet }}^{\text {indrat }}\right)$ follows from (2.1.2) (If $)$, a similar calculation shows that $R \Gamma\left(k_{\text {proet }}^{\prime}, A\right)=A\left(k^{\prime}\right)$. Hence $A$ is $\mathrm{P}$-acyclic.

For example, the proalgebraic groups $\mathbf{G}_{a}^{\mathbb{N}}, W$ and $W^{\mathbb{N}}$ are P-acyclic. The groups $\mathbb{Z}_{l}$ for a prime $l$ and $\mathbf{G}_{m}^{\mathbb{N}}$ are not.

\section{LOCAL FIELDS WITH IND-RATIONAL BASE}

Let $K$ be a complete discrete valuation field with perfect residue field $k$ of characteristic $p>0$. In this section, we view cohomology of $K$ as a complex of sheaves on Spec $k_{\text {proet }}^{\text {indrat }}$ As explained in 1.2 , the $n$-th cohomology of this complex is the pro-étale sheafification of the presheaf

$$
k^{\prime} \mapsto H^{n}\left(\mathbf{K}\left(k^{\prime}\right), A\right),
$$

where $A$ is an fppf sheaf on $K$. To study cohomology of this form, we introduce a topological space structure on the sets of rational points of schemes over the ring $\mathbf{K}\left(k^{\prime}\right)$ following Con12. We give some density and openness results using some Artin type approximation methods and results, which reduce the problems to classical results on complete discrete valuation fields.

Then we recall the relative fppf site $\operatorname{Spec} K_{\mathrm{fppf}} / k_{\mathrm{et}}^{\text {indrat }}$ and the structure morphism

$$
\pi: \operatorname{Spec} K_{\mathrm{fppf}} / k_{\mathrm{et}}^{\text {indrat }} \rightarrow \operatorname{Spec} k_{\mathrm{et}}^{\text {indrat }}
$$

from Suz13. The complex we want is defined to be the pro-étale sheafification of the derived pushforward by $\pi$.

3.1. Basic notions and properties. We denote the ring of integers of $K$ by $\mathcal{O}_{K}$. The maximal ideal of $\mathcal{O}_{K}$ is denoted by $\mathfrak{p}_{K}$. We denote by $W$ the affine ring scheme of Witt vectors of infinite length. Since $k$ is a perfect field of characteristic $p>0$, the ring $\mathcal{O}_{K}=\lim _{n \geq 0} \mathcal{O}_{K} / \mathfrak{p}_{K}^{n}$ has a canonical structure of a $W(k)$-algebra of pro-finite-length [Ser79, II, $\S 5$, Thm. 4] (which is actually finite free over $W(k)$ in the mixed characteristic case and factors as $W(k) \rightarrow k \hookrightarrow \mathcal{O}_{K}$ in the equal characteristic case). We define sheaves of rings on the site Spec $k_{\mathrm{et}}^{\text {indrat }}$ by assigning to each $k^{\prime} \in k^{\text {indrat }}$,

$$
\begin{gathered}
\mathbf{O}_{K}\left(k^{\prime}\right)=W\left(k^{\prime}\right) \hat{\otimes}_{W(k)} \mathcal{O}_{K}=\underset{n}{\lim }\left(W_{n}\left(k^{\prime}\right) \otimes_{W_{n}(k)} \mathcal{O}_{K} / \mathfrak{p}_{K}^{n}\right), \\
\mathbf{K}\left(k^{\prime}\right)=\mathbf{O}_{K}\left(k^{\prime}\right) \otimes_{\mathcal{O}_{K}} K .
\end{gathered}
$$

The Teichmüller lift gives a morphism $\omega: \mathbb{A}_{k}^{1} \hookrightarrow \mathbf{O}_{K}$. Any element of $\mathbf{K}\left(k^{\prime}\right)$ can be written as $\sum_{n \in \mathbb{Z}} \omega\left(a_{n}\right) \pi^{n}$ with a unique sequence of elements $a_{n}$ of $k^{\prime}$ whose negative terms are zero for all but finitely many $n$, where $\pi$ is a prime element of $\mathcal{O}_{K}$. It belongs to the subring $\mathbf{O}_{K}\left(k^{\prime}\right)$ if and only if $a_{n}=0$ for all $n<0$. The sheaf $\mathbf{O}_{K}$ has a subsheaf $\mathbf{O}_{K} \otimes_{\mathcal{O}_{K}} \mathfrak{p}_{K}^{n}$ of ideals for each $n \geq 0$, which we denote by $\mathbf{p}_{K}^{n}$. The ring $\mathbf{O}_{K}\left(k^{\prime}\right)$ for any $k^{\prime} \in k^{\text {indrat }}$ is $\mathbf{p}_{K}\left(k^{\prime}\right)$-adically complete Hausdorff. The sheaves of invertible elements of $\mathbf{O}_{K}$ and $\mathbf{K}$ are denoted by $\mathbf{U}_{K}$ and $\mathbf{K}^{\times}$, respectively. For $X \in \operatorname{Set}\left(k_{\mathrm{et}}^{\text {indrat }}\right)$ and a prime (hence maximal) ideal $\mathfrak{m}$ of $k^{\prime} \in k^{\text {indrat }}$, the reduction map $X\left(k^{\prime}\right) \rightarrow X\left(k^{\prime} / \mathfrak{m}\right)$ is denoted by $x \mapsto x(\mathfrak{m})$. Its kernel, when $X \in \mathrm{Ab}\left(k_{\mathrm{et}}^{\text {indrat }}\right)$, is denoted by $X(\mathfrak{m})$. Here are some properties of the rings $\mathbf{O}_{K}\left(k^{\prime}\right)$ and $\mathbf{K}\left(k^{\prime}\right)$.

Proposition (3.1.1). Let $k^{\prime} \in k^{\text {indrat }}$. 
(a) For any $\mathfrak{m} \in \operatorname{Spec} k^{\prime}$, the ideal $\mathbf{K}(\mathfrak{m})$ (resp. $\mathbf{p}_{K}\left(k^{\prime}\right)+\mathbf{O}_{K}(\mathfrak{m})$ ) is a maximal ideal of $\mathbf{K}\left(k^{\prime}\right)$ (resp. $\mathbf{O}_{K}\left(k^{\prime}\right)$ ). The map $\mathfrak{m} \mapsto \mathbf{K}(\mathfrak{m})$ (resp. $\mathfrak{m} \mapsto \mathbf{p}_{K}\left(k^{\prime}\right)+$ $\mathbf{O}_{K}(\mathfrak{m})$ ) gives a homeomorphism from $\mathrm{Spec} k^{\prime}$ onto the maximal spectrum of $\mathbf{K}\left(k^{\prime}\right)$ (resp. $\mathbf{O}_{K}\left(k^{\prime}\right)$ ).

(b) A neighborhood base of the maximal ideal $\mathbf{K}(\mathfrak{m})$ of $\mathbf{K}\left(k^{\prime}\right)$ (resp. $\mathbf{p}_{K}\left(k^{\prime}\right)+$ $\mathbf{O}_{K}(\mathfrak{m})$ of $\left.\mathbf{O}_{K}\left(k^{\prime}\right)\right)$ is given by the family of open and closed sets

$$
\operatorname{Spec} \mathbf{K}\left(k^{\prime}\right)[1 / \omega(e)]=\operatorname{Spec} \mathbf{K}\left(k^{\prime}[1 / e]\right)
$$

(resp.

$$
\left.\operatorname{Spec} \mathbf{O}_{K}\left(k^{\prime}\right)[1 / \omega(e)]=\operatorname{Spec} \mathbf{O}_{K}\left(k^{\prime}[1 / e]\right)\right)
$$

for idempotents e $\in k^{\prime} \backslash \mathfrak{m}$. In particular, any Zariski covering of $\operatorname{Spec} \mathbf{K}\left(k^{\prime}\right)$ (resp. Spec $\left.\mathbf{O}_{K}\left(k^{\prime}\right)\right)$ can be refined by a disjoint Zariski covering.

(c) Let $\pi$ be a prime element of $\mathcal{O}_{K}$. An element $\sum_{n \geq 0} \omega\left(a_{n}\right) \pi^{n}$ of $\mathbf{O}_{K}\left(k^{\prime}\right)$ (with $\left.a_{n} \in k^{\prime}\right)$ is invertible if and only if $a_{0} \in k^{\prime \times}$. An element $\sum_{n \in \mathbb{Z}} \omega\left(a_{n}\right) \pi^{n}$ of $\mathbf{K}\left(k^{\prime}\right)$ (with $a_{n}=0$ for sufficiently small $n<0$ ) is invertible if and only if some finitely many of the $a_{n}$ generate the unit ideal of $k^{\prime}$.

Proof. (国) and (b) for $\mathbf{K}\left(k^{\prime}\right)$ were proved in [Suz13, Lem. 2.5.1-2]. (To see that the map $\mathfrak{m} \mapsto \mathbf{K}(\mathfrak{m})$ is not only a bijection but also a homeomorphism, use (b).) The same proof works for $\mathbf{O}_{K}\left(k^{\prime}\right)$.

(IC) The statement for $\mathbf{O}_{K}\left(k^{\prime}\right)$ is obvious. If $\sum \omega\left(a_{n}\right) \pi^{n} \in \mathbf{K}\left(k^{\prime}\right)$ is invertible, then so is its image in the complete discrete valuation field $\mathbf{K}\left(k^{\prime} / \mathfrak{m}\right)$ for any $\mathfrak{m} \in$ Spec $k^{\prime}$, hence $a_{n} \notin \mathfrak{m}$ for some $n$. Therefore the $a_{n}$ generate the unit ideal of $k^{\prime}$. Some finite linear combination of them gives $1 \in k^{\prime}$.

For the converse, denote by $D(\omega(a))$ and $V(\omega(a))$ for $a \in k^{\prime}$ the open set $\operatorname{Spec} \mathbf{K}\left(k^{\prime}\right)[1 / \omega(a)]$ and its complementary closed set $\operatorname{Spec} \mathbf{K}\left(k^{\prime} /(a)\right)$ of $\operatorname{Spec} \mathbf{K}\left(k^{\prime}\right)$, respectively. They are both open and closed since $k^{\prime} \in k^{\text {indrat }}$ and hence $a$ is a unit times an idempotent. For any $m \in \mathbb{Z}$, on the open and closed set $U_{m}=$ $\bigcap_{l<m} V\left(\omega\left(a_{l}\right)\right) \cap D\left(\omega\left(a_{m}\right)\right)$, the element

$$
\sum \omega\left(a_{n}\right) \pi^{n} \equiv \sum_{n \geq m} \omega\left(a_{n}\right) \pi^{n}=\pi^{m} \sum_{n \geq 0} \omega\left(a_{n+m}\right) \pi^{n}
$$

becomes a unit. Some finitely many of these disjoint open and closed sets $\left\{U_{m}\right\}$ cover Spec $\mathbf{K}\left(k^{\prime}\right)$ by assumption. Hence $\sum \omega\left(a_{n}\right) \pi^{n}$ is a unit in $\mathbf{K}\left(k^{\prime}\right)$.

Some properties of schemes over the rings $\mathbf{O}_{K}\left(k^{\prime}\right), \mathbf{K}\left(k^{\prime}\right)$ :

Proposition (3.1.3). Let $k^{\prime} \in k^{\text {indrat }}$.

(a) If $X$ is an affine $\mathbf{O}_{K}\left(k^{\prime}\right)$-scheme and $x \in X\left(\mathbf{K}\left(k^{\prime}\right)\right)$, then the subset

$$
\left\{\mathfrak{m} \in \operatorname{Spec} k^{\prime} \mid x(\mathfrak{m}) \in X\left(\mathbf{O}_{K}\left(k^{\prime} / \mathfrak{m}\right)\right)\right\}
$$

of Spec $k^{\prime}$ is closed. It is open if $X$ is of finite type.

(b) Let $X$ be a separated $\mathbf{O}_{K}\left(k^{\prime}\right)$-scheme locally of finite type and $x \in X\left(\mathbf{K}\left(k^{\prime}\right)\right)$. If $x(\mathfrak{m}) \in X\left(\mathbf{O}_{K}\left(k^{\prime} / \mathfrak{m}\right)\right)$ for all $\mathfrak{m} \in$ Spec $k^{\prime}$, then $x \in X\left(\mathbf{O}_{K}\left(k^{\prime}\right)\right)$.

(c) Let $X$ be either a proper $\mathbf{O}_{K}\left(k^{\prime}\right)$-scheme or written as $\mathcal{G} \times_{\mathcal{O}_{K}} \mathbf{O}_{K}\left(k^{\prime}\right)$ with $\mathcal{G}$ the Néron model of a smooth group scheme over $K$. Then we have $X\left(\mathbf{O}_{K}\left(k^{\prime}\right)\right)=X\left(\mathbf{K}\left(k^{\prime}\right)\right)$.

(d) If $X$ is any $\mathbf{O}_{K}\left(k^{\prime}\right)$-scheme, then

$$
X\left(\mathbf{O}_{K}\left(k^{\prime}\right)\right)={\underset{n}{n}}_{\lim } X\left(\mathbf{O}_{K} / \mathbf{p}_{K}^{n}\left(k^{\prime}\right)\right)
$$


Proof. (国) Write $X=\operatorname{Spec} S$. Let $\varphi: S \rightarrow \mathbf{K}\left(k^{\prime}\right)$ be the $\mathbf{O}_{K}\left(k^{\prime}\right)$-algebra homomorphism corresponding to $x \in X\left(\mathbf{K}\left(k^{\prime}\right)\right)$. For $f \in S$, write $\varphi(f)=\sum \omega\left(a_{n, f}\right) \pi^{n}$ with $a_{n, f} \in k^{\prime}$, where $\pi$ is a prime element of $\mathcal{O}_{K}$. Then a maximal ideal $\mathfrak{m} \in \operatorname{Spec} k^{\prime}$ is in the given subset if and only if the composite $S \rightarrow \mathbf{K}\left(k^{\prime}\right) \rightarrow \mathbf{K}\left(k^{\prime} / \mathfrak{m}\right)$ factors through $\mathbf{O}_{K}\left(k^{\prime} / \mathfrak{m}\right)$. This is equivalent that $a_{n, f} \in \mathfrak{m}$ for all $n<0$ and $f \in S$. Therefore the given subset is the closed subset defined by the ideal generated by the elements $a_{n, f} \in k^{\prime}$ for all $n<0$ and $f \in S$. We may consider only those $f$ 's in any fixed set of generators of the $\mathbf{O}_{K}\left(k^{\prime}\right)$-algebra $S$. Hence the ideal is finitely generated if $S$ is finitely generated. A finitely generated ideal of $k^{\prime} \in k^{\text {indrat }}$ is generated by a single idempotent. Hence the given set is open in this case.

(b) Note that the separatedness of $X$ and the injectivity of $\mathbf{O}_{K}\left(k^{\prime}\right) \hookrightarrow \mathbf{K}\left(k^{\prime}\right)$ implies that the natural map $X\left(\mathbf{O}_{K}\left(k^{\prime}\right)\right) \rightarrow X\left(\mathbf{K}\left(k^{\prime}\right)\right)$ is injective. We first treat the case where $X$ is affine. Using the notation in the proof of the previous assertion, the assumption implies that $a_{n, f}=0$ for all $n<0$ and $f \in S$. Hence $\varphi$ factors through $\mathbf{O}_{K}\left(k^{\prime}\right)$ and therefore $x \in X\left(\mathbf{O}_{K}\left(k^{\prime}\right)\right)$ in this case.

Next we treat the general case. Let $\left\{U_{\lambda}\right\}$ be an affine open cover of $X$. For any $\mathfrak{m} \in \operatorname{Spec} k^{\prime}$, the sets $U_{\lambda}\left(\mathbf{O}_{K}\left(k^{\prime} / \mathfrak{m}\right)\right)$ indexed by $\lambda$ cover $X\left(\mathbf{O}_{K}\left(k^{\prime} / \mathfrak{m}\right)\right)$ since $\mathbf{O}_{K}\left(k^{\prime} / \mathfrak{m}\right)$ is local. Hence the assumption implies that the sets

$$
\left\{\mathfrak{m} \in \operatorname{Spec} k^{\prime} \mid x(\mathfrak{m}) \in U_{\lambda}\left(\mathbf{O}_{K}\left(k^{\prime} / \mathfrak{m}\right)\right)\right\}
$$

indexed by $\lambda$ cover Spec $k^{\prime}$. For each $\lambda$, this set is open since the affine $\mathbf{O}_{K}\left(k^{\prime}\right)$ scheme $U_{\lambda}$ is of finite type and by (国). Refine this open covering of Spec $k^{\prime}$ by a disjoint open covering $\left\{\operatorname{Spec} k^{\prime}\left[1 / e_{\lambda}\right]\right\}$ with $e_{\lambda}$ idempotents. Note that all but finitely many $e_{\lambda}$ are zero. By considering $k^{\prime}\left[1 / e_{\lambda}\right]$ for each $\lambda$ instead of $k^{\prime}$, we are reduced to the affine case.

(C) By the previous assertion, it is reduced to showing $X\left(\mathbf{O}_{K}\left(k^{\prime} / \mathfrak{m}\right)\right)=X\left(\mathbf{K}\left(k^{\prime} / \mathfrak{m}\right)\right)$ for all $\mathfrak{m} \in \operatorname{Spec} k^{\prime}$. The proper case is the valuative criterion. For the Néron case, note that $k^{\prime} / \mathfrak{m}$ is a separable (possibly transcendental) field extension of $k$ in the sense of [Bou03, Chap. V, $\S 15$, no. 2-3] by [loc. cit., no. 5, Thm. 3 b)]. Therefore [BLR90, 10.1, Prop. 3] shows that $\mathcal{G} \times{ }_{\mathcal{O}_{K}} \mathbf{O}\left(k^{\prime} / \mathfrak{m}\right)$ is the Néron model, over the discrete valuation ring $\mathbf{O}\left(k^{\prime} / \mathfrak{m}\right)$, of its generic fiber. Hence the Néron mapping property implies that $X\left(\mathbf{O}_{K}\left(k^{\prime} / \mathfrak{m}\right)\right)=X\left(\mathbf{K}\left(k^{\prime} / \mathfrak{m}\right)\right)$.

(d) This is trivial if $X$ is affine. In general, let $\left\{U_{\lambda}\right\}$ be an affine open cover of $X$. Let $\left(x_{n}\right)_{n}$ be an element of the limit in question. The pullback of $\left\{U_{\lambda}\right\}$ by the morphism $x_{1}$ : Spec $k^{\prime} \rightarrow X$ gives an open covering of Spec $k^{\prime}$. Refine it by a disjoint covering $\left\{\operatorname{Spec} k^{\prime}\left[1 / e_{\lambda}\right]\right\}$ with idempotents $e_{\lambda}$. Then $x_{1}$ restricted to $\operatorname{Spec} k^{\prime}\left[1 / e_{\lambda}\right]$ factors through $U_{\lambda}$. Since the the surjection $\mathbf{O}_{K} / \mathbf{p}_{K}^{n}\left(k^{\prime}\right) \rightarrow k^{\prime}$ has a nilpotent kernel, we know that $x_{n}$ restricted to $\operatorname{Spec} \mathbf{O}_{K} / \mathbf{p}_{K}^{n}\left(k^{\prime}\left[1 / e_{\lambda}\right]\right)$ factors through $U_{\lambda}$ for all $n$. The affine case then implies that $\left(x_{n}\right)_{n}$ comes from $X\left(\mathbf{O}_{K}\left(k^{\prime}\right)\right)$.

3.2. Topology on rational points of varieties. We give a topology on the set $X\left(\mathbf{K}\left(k^{\prime}\right)\right)$ for any $\mathbf{K}\left(k^{\prime}\right)$-scheme $X$ locally of finite type with $k^{\prime} \in k^{\text {indrat }}$ and on the set $Y\left(\mathbf{O}_{K}\left(k^{\prime}\right)\right)$ for any $\mathbf{O}_{K}\left(k^{\prime}\right)$-scheme $Y$ locally of finite type. We follow Con12, $\S 2-3]$.

First for each $k^{\prime} \in k^{\text {indrat }}$, the ring $\mathbf{O}_{K}\left(k^{\prime}\right)$ is a topological ring by the ideals $\mathbf{p}_{K}^{n}\left(k^{\prime}\right), n \geq 0$. We give a topological ring structure on $\mathbf{K}\left(k^{\prime}\right)$ so that the subring $\mathbf{O}_{K}\left(k^{\prime}\right)$ is open. Recall from [Con12, Prop. 2.1] that the set $X\left(\mathbf{K}\left(k^{\prime}\right)\right)$ for an affine $\mathbf{K}\left(k^{\prime}\right)$-scheme $X$ of finite type has a canonical structure of a topological space. Explicitly, choose an embedding $X \hookrightarrow \mathbb{A}^{n}$ for some $n$, give the product topology 
on $\mathbb{A}^{n}\left(\mathbf{K}\left(k^{\prime}\right)\right)=\mathbf{K}\left(k^{\prime}\right)^{n}$ and give the subspace topology on $X\left(\mathbf{K}\left(k^{\prime}\right)\right)$. This is independent of the choice, and relies only on the fact that $\mathbf{K}\left(k^{\prime}\right)$ is a topological ring. Similarly we have a canonical topological space structure on $Y\left(\mathbf{O}_{K}\left(k^{\prime}\right)\right)$ for an affine $\mathbf{O}_{K}\left(k^{\prime}\right)$-scheme $Y$ of finite type.

To proceed to the non-affine case, note that the subsets $\mathbf{K}^{\times}\left(k^{\prime}\right) \subset \mathbf{K}\left(k^{\prime}\right)$ and $\mathbf{U}_{K}\left(k^{\prime}\right) \subset \mathbf{O}_{K}\left(k^{\prime}\right)$ are open, and the inverse maps on them are continuous, by (3.1.1) (C) and (3.1.2). Therefore if $X$ is an affine $\mathbf{K}\left(k^{\prime}\right)$-scheme and $U$ is a basic open affine subset of $X$ (i.e., a localization by one element), then $U\left(\mathbf{K}\left(k^{\prime}\right)\right) \subset X\left(\mathbf{K}\left(k^{\prime}\right)\right)$ is an open immersion of topological spaces as explained in the proof of Con12, Prop. 3.1]. If we try to apply the whole cited proposition, we will need the equality

$$
X\left(\mathbf{K}\left(k^{\prime}\right)\right)=\bigcup_{\lambda} U_{\lambda}\left(\mathbf{K}\left(k^{\prime}\right)\right)
$$

for a $\mathbf{K}\left(k^{\prime}\right)$-scheme $X$ locally of finite type and an arbitrary affine open cover $\left\{U_{\lambda}\right\}$ of $X$, which requires that $\mathbf{K}\left(k^{\prime}\right)$ be local. When $k^{\prime} \in k^{\text {indrat }}$ is a field, then $\mathbf{K}\left(k^{\prime}\right)$ is a complete discrete valuation field, so the equality above is true. If $k^{\prime} \in k^{\text {indrat }}$ is a finite product of fields $\prod k_{i}^{\prime}$, then the above equality is not true in general, but the equality $X\left(\mathbf{K}\left(k^{\prime}\right)\right)=\prod X\left(\mathbf{K}\left(k_{i}^{\prime}\right)\right)$ gives a product topology on $X\left(\mathbf{K}\left(k^{\prime}\right)\right)$. For a general $k^{\prime}=\bigcup k_{\lambda}^{\prime} \in k^{\text {indrat }}$ with $k_{\lambda}^{\prime} \in k^{\text {rat }}$, it is not true that $\mathbf{K}\left(k^{\prime}\right)=\bigcup \mathbf{K}\left(k_{\lambda}^{\prime}\right)$, and hence it is not immediately clear how to use the topologies on the sets $U_{\lambda}\left(\mathbf{K}\left(k^{\prime}\right)\right)$ to topologize $X\left(\mathbf{K}\left(k^{\prime}\right)\right)$. The situation is the same for $\mathbf{O}_{K}\left(k^{\prime}\right)$. To topologize $X\left(\mathbf{K}\left(k^{\prime}\right)\right)$ for a general $k^{\prime} \in k^{\text {indrat }}$, we will use the following.

Proposition (3.2.1). Let $k^{\prime} \in k^{\text {indrat }}$.

(a) Let $X$ be a $\mathbf{K}\left(k^{\prime}\right)$-scheme locally of finite type and $\left\{U_{\lambda}\right\}_{\lambda \in \Lambda}$ any affine open cover of $X$. Given a family $\left\{e_{\lambda}\right\}$ of disjoint idempotents of $k^{\prime}$ indexed by $\Lambda$ such that $\sum e_{\lambda}=1$ and a family of $K$-morphisms $\operatorname{Spec} \mathbf{K}\left(k^{\prime}\left[1 / e_{\lambda}\right]\right) \rightarrow U_{\lambda}$ $(\hookrightarrow X)$, a trivial patching gives a K-morphism $\operatorname{Spec} \mathbf{K}\left(k^{\prime}\right) \rightarrow X$. The map

$$
X\left(\mathbf{K}\left(k^{\prime}\right)\right) \leftarrow \bigcup_{\left\{e_{\lambda}\right\}} \prod_{\lambda} U_{\lambda}\left(\mathbf{K}\left(k^{\prime}\left[1 / e_{\lambda}\right]\right)\right),
$$

thus obtained is bijective.

(b) Let $X$ be an affine $\mathbf{K}\left(k^{\prime}\right)$-scheme of finite type and $\left\{U_{i}\right\}$ any basic affine open finite cover of $X$. Let $\left\{e_{i}\right\}$ be a family of disjoint idempotents of $k^{\prime}$ such that $\sum e_{i}=1$. Then the bijection and the natural map

$$
X\left(\mathbf{K}\left(k^{\prime}\right)\right)=\prod X\left(\mathbf{K}\left(k^{\prime}\left[1 / e_{i}\right]\right)\right) \leftarrow \prod U_{i}\left(\mathbf{K}\left(k^{\prime}\left[1 / e_{i}\right]\right)\right)
$$

is a homeomorphism and an open immersion, respectively.

There are statements for $\mathbf{O}_{K}\left(k^{\prime}\right)$ instead of $\mathbf{K}\left(k^{\prime}\right)$ in the obvious manner.

Proof. The first assertion follows from (3.1.1) (b). To check that the bijection in the second assertion is a homeomorphism, we can use the fact that $\mathbf{K}\left(k^{\prime}\right)=$ $\prod \mathbf{K}\left(k^{\prime}\left[1 / e_{i}\right]\right)$ is a homeomorphism. The final map is an open immersion as seen before. The statements for $\mathbf{O}_{K}\left(k^{\prime}\right)$ can be proven similarly.

Given this, we introduce a topology on $X\left(\mathbf{K}\left(k^{\prime}\right)\right)$ by declaring that a subset of $X\left(\mathbf{K}\left(k^{\prime}\right)\right)$ is open if its intersection with the product topological space $\prod_{\lambda} U_{\lambda}\left(\mathbf{K}\left(k^{\prime}\left[1 / e_{\lambda}\right]\right)\right)$ is open for any $\left\{e_{\lambda}\right\}$. This is independent of the choice of the affine open covering $\left\{U_{\lambda}\right\}$ as in [Con12, Prop. 3.1]. Similarly we have a topology on $Y\left(\mathbf{O}_{K}\left(k^{\prime}\right)\right)$ for an $\mathbf{O}_{K}\left(k^{\prime}\right)$-scheme $Y$ locally of finite type. Some properties 
of the topology given in Con12 also hold for our topology. We list them. A $\mathbf{K}\left(k^{\prime}\right)$-morphism $X_{1} \rightarrow X_{2}$ induces a continuous map $X_{1}\left(\mathbf{K}\left(k^{\prime}\right)\right) \rightarrow X_{2}\left(\mathbf{K}\left(k^{\prime}\right)\right)$ for any $k^{\prime} \in k^{\text {indrat }}$. We have homeomorphisms $\mathbb{A}^{n}\left(\mathbf{K}\left(k^{\prime}\right)\right)=\mathbf{K}\left(k^{\prime}\right)^{n}$ and $\left(X_{2} \times_{X_{1}}\right.$ $\left.X_{3}\right)\left(\mathbf{K}\left(k^{\prime}\right)\right)=X_{2}\left(\mathbf{K}\left(k^{\prime}\right)\right) \times_{X_{1}\left(\mathbf{K}\left(k^{\prime}\right)\right)} X_{3}\left(\mathbf{K}\left(k^{\prime}\right)\right)$. A closed (resp. open) immersion $X_{1} \hookrightarrow X_{2}$ corresponds to a closed (resp. open) immersion $X_{1}\left(\mathbf{K}\left(k^{\prime}\right)\right) \hookrightarrow X_{2}\left(\mathbf{K}\left(k^{\prime}\right)\right)$. If $X$ is separated, then $X\left(\mathbf{K}\left(k^{\prime}\right)\right)$ is Hausdorff. Similar for $\mathbf{O}_{K}\left(k^{\prime}\right)$.

We need the following three topological propositions.

Proposition (3.2.2). Let $k^{\prime} \in k^{\text {indrat }}$. Let $X$ be a separated $\mathbf{O}_{K}\left(k^{\prime}\right)$-scheme locally of finite type. Then $X\left(\mathbf{O}_{K}\left(k^{\prime}\right)\right)$ is an open subset of $X\left(\mathbf{K}\left(k^{\prime}\right)\right)$.

Proof. We may assume that $X$ is affine. Let $X \hookrightarrow \mathbb{A}^{n}$ be a closed immersion. Then $X\left(\mathbf{O}_{K}\left(k^{\prime}\right)\right)=X\left(\mathbf{K}\left(k^{\prime}\right)\right) \cap \mathbb{A}^{n}\left(\mathbf{O}_{K}\left(k^{\prime}\right)\right)$ in $\mathbb{A}^{n}\left(\mathbf{K}\left(k^{\prime}\right)\right)$. Since $\mathbf{O}_{K}\left(k^{\prime}\right)$ is open in $\mathbf{K}\left(k^{\prime}\right)$ by definition, it follows that $X\left(\mathbf{O}_{K}\left(k^{\prime}\right)\right)$ is open in $X\left(\mathbf{K}\left(k^{\prime}\right)\right)$.

Before the next proposition, recall from $\$ 2.4$ that

$$
\mathbf{K}^{\mathrm{fp}}\left(k^{\prime}\right)=\underset{\lim _{\longrightarrow}}{\mathbf{K}}\left(k_{\lambda}^{\prime}\right), \quad \mathbf{O}_{K}^{\mathrm{fp}}\left(k^{\prime}\right)=\lim _{\longrightarrow} \mathbf{O}_{K}\left(k_{\lambda}^{\prime}\right)
$$

for $k^{\prime}=\bigcup k_{\lambda}^{\prime} \in k^{\text {indrat }}$ with $k_{\lambda}^{\prime} \in k^{\text {rat }}$. The first (resp. second) ring is a filtered union of finite products of complete discrete valuation fields (resp. rings). When $k^{\prime}=\bar{k}$ (and hence the $k_{\lambda}^{\prime}$ are finite extensions of $k$ ), the $K$-algebra $\mathbf{K}^{\mathrm{fp}}\left(k^{\prime}\right)$ is the maximal unramified extension $K^{\mathrm{ur}}$ of $K$, whose completion is $\hat{K}^{\mathrm{ur}}=\mathbf{K}(\bar{k})$ 回

Proposition (3.2.3). Let $X$ be a $K$-scheme locally of finite type and $k^{\prime} \in k^{\text {indrat }}$. Then $X\left(\mathbf{K}^{\mathrm{fp}}\left(k^{\prime}\right)\right)$ is a dense subset of $X\left(\mathbf{K}\left(k^{\prime}\right)\right)$. There is a similar statement for $\mathcal{O}_{K}$ and $\mathbf{O}_{K}$ in place of $K$ and $\mathbf{K}$, respectively.

Proof. The ring $\mathbf{K}\left(k^{\prime}\right)$ is faithfully flat over $\mathbf{K}^{\mathrm{fp}}\left(k^{\prime}\right)$ and the map $X\left(\mathbf{K}^{\mathrm{fp}}\left(k^{\prime}\right)\right) \rightarrow$ $X\left(\mathbf{K}\left(k^{\prime}\right)\right)$ is injective. We may assume that $X$ is affine and defined over $\mathcal{O}_{K}$. The map $X\left(\mathbf{O}_{K}^{\mathrm{fp}}\left(k^{\prime}\right)\right) \rightarrow X\left(\mathbf{O}_{K}\left(k^{\prime}\right)\right)$ is injective by the same reason. By scaling, it is enough to show that $X\left(\mathbf{O}_{K}^{\mathrm{fp}}\left(k^{\prime}\right)\right)$ is dense in $X\left(\mathbf{O}_{K}\left(k^{\prime}\right)\right)$.

Recall Greenberg's approximation theorem [Gre66, Cor. 1 to Thm. 1]: there are integers $N \geq 1, c \geq 1, s \geq 0$ such that for any $n \geq N$ and any $x \in X\left(\mathcal{O}_{K} / \mathfrak{p}_{K}^{n}\right)$, the image of $x$ in $X\left(\mathcal{O}_{K} / \mathfrak{p}_{K}^{\lceil n / c\rceil-s}\right)$ lifts to $X\left(\mathcal{O}_{K}\right)$. The proof of this theorem works for the following slightly stronger statement: there are integers $N \geq 1, c \geq 1, s \geq 0$ such that for any perfect field $k^{\prime \prime}$ over $k$, any $n \geq N$ and any $x \in X\left(\mathbf{O}_{K}\left(k^{\prime \prime}\right) / \mathbf{p}_{K}^{n}\left(k^{\prime \prime}\right)\right)$, the image of $x$ in $X\left(\mathbf{O}_{K}\left(k^{\prime \prime}\right) / \mathbf{p}_{K}^{\lceil n / c\rceil-s}\left(k^{\prime \prime}\right)\right)$ lifts to $X\left(\mathbf{O}_{K}\left(k^{\prime \prime}\right)\right)$. (Note that $\mathbf{O}_{K}\left(k^{\prime \prime}\right)$ is a complete discrete valuation ring in which a prime element of $\mathcal{O}_{K}$ remains prime.)

Now let $n \geq N$ and $x \in X\left(\mathbf{O}_{K}\left(k^{\prime}\right)\right)$. Write $k^{\prime}=\bigcup k_{\lambda}^{\prime}$ with $k_{\lambda}^{\prime} \in k^{\text {rat }}$. Since $\left(\mathbf{O}_{K} / \mathbf{p}_{K}^{n}\right)^{\mathrm{fp}}\left(k^{\prime}\right)=\mathbf{O}_{K} / \mathbf{p}_{K}^{n}\left(k^{\prime}\right)=\mathbf{O}_{K}\left(k^{\prime}\right) / \mathbf{p}_{K}^{n}\left(k^{\prime}\right)$, the image of $x$ in $X\left(\mathbf{O}_{K} / \mathbf{p}_{K}^{n}\left(k^{\prime}\right)\right)$ belongs to $X\left(\mathbf{O}_{K} / \mathbf{p}_{K}^{n}\left(k_{\lambda}^{\prime}\right)\right)$ for some $\lambda$. Since $k_{\lambda}^{\prime}$ is a finite product of perfect fields over $k$, the image of $x$ in

$$
X\left(\mathbf{O}_{K} / \mathbf{p}_{K}^{\lceil n / c\rceil-s}\left(k_{\lambda}^{\prime}\right)\right) \subset X\left(\mathbf{O}_{K} / \mathbf{p}_{K}^{\lceil n / c\rceil-s}\left(k^{\prime}\right)\right)
$$

lifts to

$$
X\left(\mathbf{O}_{K}\left(k_{\lambda}^{\prime}\right)\right) \subset X\left(\mathbf{O}_{K}^{\mathrm{fp}}\left(k^{\prime}\right)\right) .
$$

\footnotetext{
${ }^{9}$ Do not confuse $\mathbf{O}_{K}^{\mathrm{fp}}\left(k^{\prime}\right)$ with the uncompleted tensor product $W\left(k^{\prime}\right) \otimes_{W(k)} \mathcal{O}_{K}$. If $K$ has mixed characteristic (and hence $\mathcal{O}_{K}$ is finite free over $\left.W(k)\right)$ and $k^{\prime}=\bar{k}$, then the former is $\mathcal{O}_{K}^{\text {ur }}$, which is smaller than the latter $\hat{\mathcal{O}}_{K}^{\text {ur }}$. If $K$ has equal characteristic and $k^{\prime}$ is (the perfection of) $k(x)$, then the former is $k(x)[[T]]$, which is bigger than latter $k(x) \otimes_{k}(k[[T]])$.
} 
This shows that $X\left(\mathbf{O}_{K}^{\mathrm{fp}}\left(k^{\prime}\right)\right)$ is dense in $X\left(\mathbf{O}_{K}\left(k^{\prime}\right)\right)$.

Proposition (3.2.4). Let $k^{\prime} \in k^{\text {indrat }}$ and $X, Y \mathbf{K}\left(k^{\prime}\right)$-schemes locally of finite presentation. If $f: Y \rightarrow X$ is a smooth $\mathbf{K}\left(k^{\prime}\right)$-morphism, then the image of $Y\left(\mathbf{K}\left(k^{\prime}\right)\right)$ under $f$ is an open subset of $X\left(\mathbf{K}\left(k^{\prime}\right)\right)$.

Proof. This is obvious if $Y$ is an affine space over $X$. Hence we may assume that $f$ is étale. We may further assume that $X$ and $Y$ are affine. Let $y \in Y\left(\mathbf{K}\left(k^{\prime}\right)\right)$ and $x=f(y)$. We want to show that any elements $x^{\prime}$ of $X\left(\mathbf{K}\left(k^{\prime}\right)\right)$ sufficiently close to $x$ come from $Y\left(\mathbf{K}\left(k^{\prime}\right)\right)$. For this, we are going to apply Tougéron's lemma Art69, Lem. 5.10] to solve the equation $f\left(y^{\prime}\right)=x^{\prime}$ for an unknown $y^{\prime} \in Y\left(\mathbf{K}\left(k^{\prime}\right)\right)$. This lemma is true for any Henselian pair, and the pair $\left(\mathbf{O}_{K}\left(k^{\prime}\right), \mathbf{p}_{K}\left(k^{\prime}\right)\right)$ is complete and hence Henselian. To apply the lemma, we need to write down all the conditions as polynomial equations and approximate solutions.

Let $f^{\prime}: Y^{\prime} \rightarrow X^{\prime}$ be a morphism between affine $\mathbf{O}_{K}\left(k^{\prime}\right)$-schemes of finite presentation whose base change to $\mathbf{K}\left(k^{\prime}\right)$ is $f$. Embed $X^{\prime}$ and $Y^{\prime}$ to affine spaces $\mathbb{A}_{\mathbf{O}_{K}\left(k^{\prime}\right)}^{m}$ and $\mathbb{A}_{\mathbf{O}_{K}\left(k^{\prime}\right)}^{n}$ over $\mathbf{O}_{K}\left(k^{\prime}\right)$, respectively, and extend $f^{\prime}$ to a morphism $\mathbb{A}_{\mathbf{O}_{K}\left(k^{\prime}\right)}^{n} \rightarrow \mathbb{A}_{\mathbf{O}_{K}\left(k^{\prime}\right)}^{m}$. By scaling, we may assume that $y \in Y^{\prime}\left(\mathbf{O}_{K}\left(k^{\prime}\right)\right)$.

Let $P=\left(P_{1}, \ldots, P_{l}\right)$ be a polynomial system defining $Y^{\prime} \subset \mathbb{A}_{\mathbf{O}_{K}\left(k^{\prime}\right)}^{n}$. Let $\pi$ be a prime element of $\mathcal{O}_{K}$. Since $f: Y \rightarrow X$ is étale, the differential module $\Omega_{Y^{\prime} / X^{\prime}}^{1}$ is killed by a power $\pi^{r}$ of $\pi$. We want to show that any element $x^{\prime} \in X^{\prime}\left(\mathbf{O}_{K}\left(k^{\prime}\right)\right) \subset$ $\mathbf{O}_{K}\left(k^{\prime}\right)^{m}$ with term-wise difference

$$
x^{\prime}-x \in \mathbf{p}_{K}^{2 r n+1}\left(k^{\prime}\right)^{m}
$$

comes from $Y^{\prime}\left(\mathbf{O}_{K}\left(k^{\prime}\right)\right)$, where $\mathbf{p}_{K}^{2 r n+1}\left(k^{\prime}\right)^{m} \subset \mathbf{O}_{K}\left(k^{\prime}\right)^{m}$ is the set-theoretic product of $m$ copies of the $(2 r n+1)$-st power of the ideal $\mathbf{p}_{K}\left(k^{\prime}\right)$. Let $f^{\prime-1}\left(x^{\prime}\right)$ be the fiber of $f^{\prime}: Y^{\prime} \rightarrow X^{\prime}$ over $x^{\prime} \in X^{\prime}\left(\mathbf{O}_{K}\left(k^{\prime}\right)\right)$. Then $\Omega_{f^{\prime-1}\left(x^{\prime}\right) / \mathbf{O}_{K}\left(k^{\prime}\right)}^{1}$ is killed by $\pi^{r}$. We have a commutative diagram with cartesian squares of finitely presented $\mathbf{O}_{K}\left(k^{\prime}\right)$-schemes

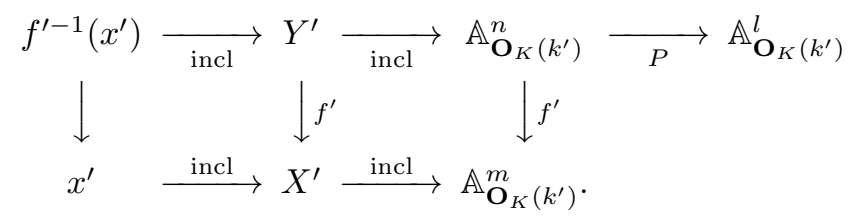

Let $y^{\prime}=\left(y_{1}^{\prime}, \ldots, y_{n}^{\prime}\right)$ be the coordinates of $\mathbb{A}_{\mathbf{O}_{K}\left(k^{\prime}\right)}^{n}$. We view $f^{\prime}: \mathbb{A}_{\mathbf{O}_{K}\left(k^{\prime}\right)}^{n} \rightarrow$ $\mathbb{A}_{\mathbf{O}_{K}\left(k^{\prime}\right)}^{m}$ as a system of $m$ polynomials in the $n$ variables $\left(y_{1}^{\prime}, \ldots, y_{n}^{\prime}\right)$ and $x^{\prime}$ as an element of $\mathbf{O}_{K}\left(k^{\prime}\right)^{m}$ (or $m$ constant polynomials). Define

$$
S=\mathbf{O}_{K}\left(k^{\prime}\right)\left[y^{\prime}\right] /\left(f^{\prime}\left(y^{\prime}\right)-x^{\prime}, P\left(y^{\prime}\right)\right)
$$

where the ideal on the right is generated by all the $m+l$ polynomials in the polynomial system $\left(f^{\prime}\left(y^{\prime}\right)-x^{\prime}, P\left(y^{\prime}\right)\right)$. We have $f^{\prime-1}\left(x^{\prime}\right)=\operatorname{Spec} S$. Let $J$ be the Jacobian matrix of the system $\left(f^{\prime}\left(y^{\prime}\right)-x^{\prime}, P\left(y^{\prime}\right)\right)$, which has entries in $\mathbf{O}_{K}\left(k^{\prime}\right)\left[y^{\prime}\right]$. Then $\Omega_{f^{\prime-1}\left(x^{\prime}\right) / \mathbf{O}_{K}\left(k^{\prime}\right)}^{1}$ is given by the cokernel of the $S$-module homomorphism $S^{m+l} \rightarrow S^{n}$ corresponding to $J$. Let $F$ be the ideal of $\mathbf{O}_{K}\left(k^{\prime}\right)\left[y^{\prime}\right]$ generated by all minors of size $n$ of $J$. Then the image $\bar{F}$ of $F$ in $S$ is the zeroth Fitting ideal of $\Omega_{f^{\prime-1}\left(x^{\prime}\right) / \mathbf{O}_{K}\left(k^{\prime}\right)}^{1}$ Lan02, XIX, $\left.\S 2\right]$. Therefore $\bar{F}$ contains the $n$-th power of the annihilator of $\Omega_{f^{\prime-1}\left(x^{\prime}\right) / \mathbf{O}_{K}\left(k^{\prime}\right)}^{1}$ by Lan02, XIX, $\S 2$, Prop. 2.5] and hence contains $\pi^{r n}$. Hence

$$
\pi^{r n} \in F+\left(f^{\prime}\left(y^{\prime}\right)-x^{\prime}, P\left(y^{\prime}\right)\right) \subset \mathbf{O}_{K}\left(k^{\prime}\right)\left[y^{\prime}\right] .
$$


Evaluate this at $y^{\prime}=y$. Since $y \in Y^{\prime}\left(\mathbf{O}_{K}\left(k^{\prime}\right)\right)$, we have $P(y)=0$. Also $f^{\prime}(y)=x$. Hence

$$
\pi^{r n} \in F(y)+\left(x-x^{\prime}\right) \subset \mathbf{O}_{K}\left(k^{\prime}\right),
$$

where $F(y)$ is the image of $F$ under the evaluation map $\mathbf{O}_{K}\left(k^{\prime}\right)\left[y^{\prime}\right] \rightarrow \mathbf{O}_{K}\left(k^{\prime}\right)$ at $y$. With this and using (3.2.5), we know that $\pi^{r n} \in F(y)$. By (3.2.7) below, we may assume that the value $\delta(y)$ of a single minor $\delta$ divides $\pi^{r n}$ in $\mathbf{O}_{K}\left(k^{\prime}\right)$, so $\mathbf{p}_{K}^{r n}\left(k^{\prime}\right) \subset \delta(y) \mathbf{O}_{K}\left(k^{\prime}\right)$. Hence

$$
\mathbf{p}_{K}^{2 r n+1}\left(k^{\prime}\right) \subset \delta(y)^{2} \mathbf{p}_{K}\left(k^{\prime}\right) .
$$

Now we have

$$
f^{\prime}(y)-x^{\prime} \in \delta(y)^{2} \mathbf{p}_{K}\left(k^{\prime}\right)^{m}, \quad P(y)=0
$$

by (3.2.5) and (3.2.6). Hence the system $\left(f^{\prime}\left(y^{\prime}\right)-x^{\prime}, P\right)$ has a root $y^{\prime} \in \mathbf{O}_{K}\left(k^{\prime}\right)^{n}$ by Tougéron's lemma [Art69, Lem. 5.10]. For this $y^{\prime}$, we have $f^{\prime}\left(y^{\prime}\right)=x^{\prime}$ and $y^{\prime} \in Y^{\prime}\left(\mathbf{O}_{K}\left(k^{\prime}\right)\right)$.

Lemma (3.2.7). If the ideal of $\mathbf{O}_{K}\left(k^{\prime}\right)$ generated by $q$ elements $\delta_{1}, \ldots, \delta_{q} \in$ $\mathbf{O}_{K}\left(k^{\prime}\right)$ contains $\pi^{s}$, then there exists a disjoint Zariski covering

$$
\operatorname{Spec} k^{\prime}=\bigsqcup_{i=1}^{q} \operatorname{Spec} k^{\prime}\left[1 / e_{i}\right]
$$

with idempotents $e_{i}$ such that the image of $\delta_{i}$ in $\mathbf{O}_{K}\left(k^{\prime}\left[1 / e_{i}\right]\right)$ divides $\pi^{s}$ for each $i$.

Proof. Let $v$ be the normalized valuation of $\mathcal{O}_{K}$. For each $\mathfrak{m} \in \operatorname{Spec} k^{\prime}$, the ideal of $\mathbf{O}_{K}\left(k^{\prime} / \mathfrak{m}\right)$ generated by $\delta_{1}(\mathfrak{m}), \ldots, \delta_{q}(\mathfrak{m})$ contains $\pi^{s}$. Therefore the sets

$$
U_{i}=\left\{\mathfrak{m} \in \operatorname{Spec} k^{\prime} \mid v\left(\delta_{i}(\mathfrak{m})\right) \leq s\right\}
$$

for $i=1, \ldots q$ cover Spec $k^{\prime}$. For each $i$, if $\delta_{i}=\sum_{n} \omega\left(a_{i, n}\right) \pi^{n}$, then $U_{i}$ is the union of the open sets Spec $k^{\prime}\left[1 / a_{i, s}\right]$, Spec $k^{\prime}\left[1 / a_{i, s-1}\right], \ldots$ Hence $\left\{U_{i}\right\}$ is an open covering of Spec $k^{\prime}$. Refine it by a disjoint Zariski covering Spec $k^{\prime}=\sqcup_{i=1}^{q} \operatorname{Spec} k^{\prime}\left[1 / e_{i}\right]$ with idempotents $e_{i}$. This choice does the job.

Now we can prove the following proposition. This is useful especially to prove that the first cohomology of an abelian variety over $K$ is ind-algebraic and all of the higher cohomology is zero as sheaves over $k$. The proof below and the use of the above topological statements are inspired by the proof of Čes15, Prop. 3.5 (a) and Lem. 5.3].

Proposition (3.2.8). Let $A$ be a smooth group scheme over $K$ and $k^{\prime} \in k^{\text {indrat }}$. Then we have

$$
H^{n}\left(\mathbf{K}\left(k^{\prime}\right)_{\mathrm{et}}, A\right)=H^{n}\left(\mathbf{K}^{\mathrm{fp}}\left(k^{\prime}\right)_{\mathrm{et}}, A\right)
$$

for any $n \geq 1$. This group is torsion.

Proof. Let $f: \operatorname{Spec} \mathbf{K}\left(k^{\prime}\right) \rightarrow \operatorname{Spec} \mathbf{K}^{\mathrm{fp}}\left(k^{\prime}\right)$ be the natural morphism. Then

$$
f_{*}: \operatorname{Ab}\left(\mathbf{K}\left(k^{\prime}\right)_{\mathrm{et}}\right) \rightarrow \operatorname{Ab}\left(\mathbf{K}^{\mathrm{fp}}\left(k^{\prime}\right)_{\mathrm{et}}\right)
$$

is exact by [Suz13, Lem. 2.5.5], hence $H^{n}\left(\mathbf{K}\left(k^{\prime}\right)_{\text {et }}, A\right)=H^{n}\left(\mathbf{K}^{\mathrm{fp}}\left(k^{\prime}\right)_{\text {et }}, f_{*} A\right)$. Note that $\mathbf{K}^{\mathrm{fp}}\left(k^{\prime}\right)$ is a filtered union of finite products of complete discrete valuation fields. The étale cohomology of a field can be calculated by Galois cohomology 
or Čech cohomology. Hence the cohomology of $\mathbf{K}^{\mathrm{fp}}\left(k^{\prime}\right)$ can be calculated by Čech cohomology. The isomorphism to be proved is thus

$$
\lim _{L^{\prime} / \underset{\mathbf{K}^{\mathrm{fp}}}{\left(k^{\prime}\right)}} \check{H}^{n}\left(L^{\prime} / \mathbf{K}^{\mathrm{fp}}\left(k^{\prime}\right), f_{*} A\right)=\underset{L^{\prime} / \lim _{\mathbf{K}^{\mathrm{fp}}}}{\left.k^{\prime}\right)} \check{H}^{n}\left(L^{\prime} / \mathbf{K}^{\mathrm{fp}}\left(k^{\prime}\right), A\right),
$$

where $L^{\prime}$ runs through faithfully flat étale $\mathbf{K}^{\mathrm{fp}}\left(k^{\prime}\right)$-algebras and $\check{H}$ denotes Čech cohomology. It is enough to prove the isomorphism for each $L^{\prime}$ before passing to the limit:

$$
\check{H}^{n}\left(L^{\prime} / \mathbf{K}^{\mathrm{fp}}\left(k^{\prime}\right), f_{*} A\right)=\check{H}^{n}\left(L^{\prime} / \mathbf{K}^{\mathrm{fp}}\left(k^{\prime}\right), A\right) .
$$

Write $k^{\prime}=\bigcup k_{\lambda}^{\prime}$ with $k_{\lambda}^{\prime} \in k^{\text {rat }}$. For some $\lambda$, such $L^{\prime}$ can be written as $L \otimes_{\mathbf{K}\left(k_{\lambda}^{\prime}\right)}$ $\mathbf{K}^{\mathrm{fp}}\left(k^{\prime}\right)$ for some faithfully flat étale $\mathbf{K}\left(k_{\lambda}^{\prime}\right)$-algebra $L$. Let $g$ : $\operatorname{Spec} \mathbf{K}\left(k^{\prime}\right) \rightarrow \operatorname{Spec} \mathbf{K}\left(k_{\lambda}^{\prime}\right)$ and $h: \operatorname{Spec} \mathbf{K}^{\mathrm{fp}}\left(k^{\prime}\right) \rightarrow \operatorname{Spec} \mathbf{K}\left(k_{\lambda}^{\prime}\right)$ be the natural morphisms. Then the Cech complex of $L^{\prime} / \mathbf{K}^{\mathrm{fp}}\left(k^{\prime}\right)$ with values in $f_{*} A$ (resp. $A$ ) is the Cech complex of $L / \mathbf{K}\left(k_{\lambda}^{\prime}\right)$ with values in $g_{*} A$ (resp. $h_{*} A$ ). Hence the isomorphism to be proven is

$$
\check{H}^{n}\left(L / \mathbf{K}\left(k_{\lambda}^{\prime}\right), g_{*} A\right)=\check{H}^{n}\left(L / \mathbf{K}\left(k_{\lambda}^{\prime}\right), h_{*} A\right) .
$$

By replacing $k_{\lambda}^{\prime}$ with $k$, it is enough to show that

$$
\check{H}^{n}\left(L / K, g_{*} A\right)=\check{H}^{n}\left(L / K, h_{*} A\right)
$$

(for any perfect field $k$ ), where $L$ is a finite Galois extension of $K$ and $g$ : $\operatorname{Spec} \mathbf{K}\left(k^{\prime}\right) \rightarrow$ Spec $K, h: \operatorname{Spec} \mathbf{K}^{\mathrm{fp}}\left(k^{\prime}\right) \rightarrow$ Spec $K$ the natural morphisms.

Let $C^{n}$ be the Weil restriction of $A$ from the $(n+1)$-fold tensor product $L \otimes_{K}$ $\cdots \otimes_{K} L$ to $K$, which is representable by a smooth $K$-scheme. The Čech complex of $L / K$ with coefficients in $g_{*} A$ (resp. $\left.h_{*} A\right)$ is the $\mathbf{K}\left(k^{\prime}\right)$-valued (resp. $\mathbf{K}^{\mathrm{fp}}\left(k^{\prime}\right)$-valued) points of the complex $\left\{C^{n}\right\}$ of group schemes over $K$ with the usual coboundary maps $\left\{d^{n}\right\}$. Let $Z^{n}$ be the kernel of $d^{n}: C^{n} \rightarrow C^{n+1}$, which is a $K$-scheme locally of finite type. We know that $d^{n-1}: C^{n-1} \rightarrow Z^{n}$ is a smooth morphism as shown in the proof of [Mil80, III, Thm. 3.9]. Consider the commutative diagram

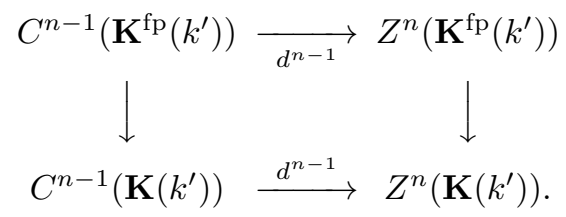

The vertical maps are injective with dense image by (3.2.3). The horizontal ones have open image by (3.2.4). Therefore the map $\check{H}^{n}\left(L / K, h_{*} A\right) \rightarrow \check{H}^{n}\left(L / K, g_{*} A\right)$ induced on the cokernels of the horizontal maps is an isomorphism.

Galois cohomology is torsion in positive degrees. Hence $\check{H}^{n}\left(L / K, h_{*} A\right)$ is torsion (killed by $[L: K])$, and $H^{n}\left(\mathbf{K}^{\mathrm{fp}}\left(k^{\prime}\right)_{\mathrm{et}}, A\right)$ is torsion.

The quotient in the following proposition will appear in the next subsection as the first cohomology of $\mathcal{O}_{K}$ with support on the closed point.

Proposition (3.2.9). Let $A$ be a separated group scheme locally of finite type over $\mathcal{O}_{K}$ and $k^{\prime} \in k^{\text {indrat }}$. Then we have

$$
A\left(\mathbf{K}\left(k^{\prime}\right)\right) / A\left(\mathbf{O}_{K}\left(k^{\prime}\right)\right)=A\left(\mathbf{K}^{\mathrm{fp}}\left(k^{\prime}\right)\right) / A\left(\mathbf{O}_{K}^{\mathrm{fp}}\left(k^{\prime}\right)\right) .
$$


Proof. Consider the commutative diagram

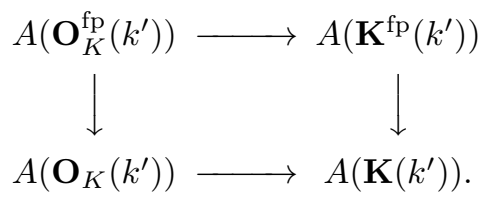

As in the proof of the previous proposition, the vertical maps are injective with dense image. The horizontal inclusions are open immersions by (3.2.2). Hence the map induced on the cokernels of the horizontal maps is an isomorphism.

We will use the following to see that the group $N\left(\mathcal{O}_{K}\right)$ for a finite flat group scheme $N$ over $\mathcal{O}_{K}$ can be viewed as an étale group over $k$. A direct algebraic proof is not difficult, but a proof using topology is clearer.

Proposition (3.2.10). Let $X$ be a locally quasi-finite separated $\mathcal{O}_{K}$-scheme with locally finite part $X^{f}$. Let $K^{\mathrm{ur}}$ be a maximal unramified extension of $K$. Let $x$ and $x^{f}$ be the sets $X\left(K^{\mathrm{ur}}\right)$ and $X^{f}\left(K^{\mathrm{ur}}\right)$, respectively, viewed as étale schemes over $k$. Then $X\left(\mathbf{O}_{K}\left(k^{\prime}\right)\right)=x^{f}\left(k^{\prime}\right)$ and $X\left(\mathbf{K}\left(k^{\prime}\right)\right)=x\left(k^{\prime}\right)$ for any $k^{\prime} \in k^{\text {indrat }}$.

Proof. Note that $X$ is a disjoint union of the Spec's of finite local $\mathcal{O}_{K}$-algebras and/or finite local $K$-algebras. Write $X=X^{f} \sqcup Y$, where $Y$ is a locally finite $K$-scheme. We first show that for any $k^{\prime} \in k^{\text {indrat}}$, any $\mathcal{O}_{K}$-scheme morphism Spec $\mathbf{O}_{K}\left(k^{\prime}\right) \rightarrow X$ factors through $X^{f}$. By (3.2.1) (国), we can write $k^{\prime}=k_{1}^{\prime} \times k_{2}^{\prime}$ with $k_{1}^{\prime}, k_{2}^{\prime} \in k^{\text {indrat }}$ such that the morphism Spec $\mathbf{O}_{K}\left(k^{\prime}\right) \rightarrow X$ is the disjoint union of morphisms Spec $\mathbf{O}_{K}\left(k_{1}^{\prime}\right) \rightarrow X^{f}$ and $\operatorname{Spec} \mathbf{O}_{K}\left(k_{2}^{\prime}\right) \rightarrow Y$. Then $\mathbf{O}_{K}\left(k_{2}^{\prime}\right)$ has to be a $K$-algebra. Therefore if $k_{2}^{\prime}$ were non-zero, then the discrete valuation ring $\mathbf{O}_{K}\left(k_{2}^{\prime} / \mathfrak{m}\right)$ for $\mathfrak{m} \in \operatorname{Spec} k_{2}^{\prime}$ would have to be a $K$-algebra, which is absurd. Hence $k_{2}^{\prime}=0$, and $\operatorname{Spec} \mathbf{O}_{K}\left(k^{\prime}\right) \rightarrow X$ factors through $X^{f}$.

Hence $X\left(\mathbf{O}_{K}\left(k^{\prime}\right)\right)=X^{f}\left(\mathbf{O}_{K}\left(k^{\prime}\right)\right)$. This is further equal to $X^{f}\left(\mathbf{K}\left(k^{\prime}\right)\right)$ by (3.1.3) (ㄷ). Hence the first statement $X\left(\mathbf{O}_{K}\left(k^{\prime}\right)\right)=X^{f}\left(\mathbf{K}\left(k^{\prime}\right)\right)=x^{f}\left(k^{\prime}\right)$ is reduced to the second $X\left(\mathbf{K}\left(k^{\prime}\right)\right)=x\left(k^{\prime}\right)$.

To prove $X\left(\mathbf{K}\left(k^{\prime}\right)\right)=x\left(k^{\prime}\right)$, we may assume that $X$ is connected by (3.2.1) (国). We may further assume that $X$ is reduced, so $X=\operatorname{Spec} L$ for some finite extension $L / K$. Let $k_{L}$ be the residue field of $L$. Then $x=\operatorname{Spec} k_{L}$ if $L / K$ is unramified and $x=\emptyset$ otherwise. We also have $\operatorname{Hom}_{K}\left(L, \mathbf{K}^{\mathrm{fp}}\left(k^{\prime}\right)\right)=\operatorname{Hom}_{k}\left(k_{L}, k^{\prime}\right)$ if $L / K$ is unramified and $\operatorname{Hom}_{K}\left(L, \mathbf{K}^{\mathrm{fp}}\left(k^{\prime}\right)\right)=\operatorname{Hom}_{k}\left(0, k^{\prime}\right)$ otherwise. Hence $X\left(\mathbf{K}^{\mathrm{fp}}\left(k^{\prime}\right)\right)=$ $x\left(k^{\prime}\right)$, which is finite discrete. Since $X$ is separated, the topological space $X\left(\mathbf{K}\left(k^{\prime}\right)\right)$ is Hausdorff. By (3.2.3), we know that $X\left(\mathbf{K}^{\mathrm{fp}}\left(k^{\prime}\right)\right)$ is dense in $X\left(\mathbf{K}\left(k^{\prime}\right)\right)$. Therefore $X\left(\mathbf{K}\left(k^{\prime}\right)\right)=X\left(\mathbf{K}^{\mathrm{fp}}\left(k^{\prime}\right)\right)=x\left(k^{\prime}\right)$.

3.3. The relative fppf site of a local field. We recall from [Suz13, §2.3-4] the relative fppf site of $K$ over $k$, the fppf structure morphism and the cup product formalism in this setting. We provide more on cohomology of $\mathcal{O}_{K}$ with or without support. The site used in [Suz13, loc. cit.] was Spec $k_{\mathrm{et}}^{\text {indrat }}$. We will apply pro-étale sheafification to obtain a similar formalism over $\operatorname{Spec} k_{\text {proet }}^{\text {indrat }}$.

Let $K / k^{\text {indrat }}$ be the category of pairs $\left(S, k_{S}\right)$, where $k_{S} \in k^{\text {indrat }}$ and $S$ a finitely presented $\mathbf{K}\left(k_{S}\right)$-algebra. A morphism $\left(S, k_{S}\right) \rightarrow\left(S^{\prime}, k_{S^{\prime}}\right)$ consists of a morphism 
$k_{S} \rightarrow k_{S^{\prime}}$ in $k^{\text {indrat }}$ and a ring homomorphism $S \rightarrow S^{\prime}$ such that the diagram

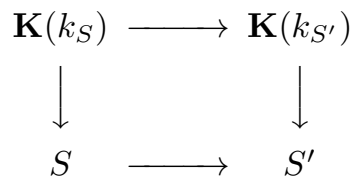

commutes. The composite of two morphisms is defined in the obvious way. We say that a morphism $\left(S, k_{S}\right) \rightarrow\left(S^{\prime}, k_{S^{\prime}}\right)$ is flat/étale if $k_{S} \rightarrow k_{S^{\prime}}$ is étale and $S \rightarrow S^{\prime}$ is flat. We endow the category $K / k^{\text {indrat }}$ with the topology where a covering of an object $\left(S, k_{S}\right)$ is a finite family $\left\{\left(S_{i}, k_{i}\right)\right\}$ of objects flat/étale over $\left(S, k_{S}\right)$ such that $\prod S_{i}$ is faithfully flat over $S$. The resulting site is the relative fppf site Spec $K_{\mathrm{fppf}} / k_{\mathrm{et}}^{\text {indrat }}$ of $K$ over $k$ [Suz13, Def. 2.3.2]. The cohomology of an object $\left(S, k_{S}\right)$ with coefficients in $A \in \mathrm{Ab}\left(K_{\mathrm{fppf}} / k_{\mathrm{et}}^{\text {indrat }}\right)$ is given by the fppf cohomology of $S$ :

$$
R \Gamma\left(\left(S, k_{S}\right), A\right)=R \Gamma\left(S_{\mathrm{fppf}}, f_{*} A\right),
$$

where $f:$ Spec $K_{\mathrm{fppf}} / k_{\mathrm{et}}^{\text {indrat }} \rightarrow$ Spec $S_{\mathrm{fppf}}$ is given by the functor sending a finitely presented $S$-algebra $S^{\prime}$ to the object $\left(S^{\prime}, k_{S}\right)$ (Suz13, Prop. 2.3.4]). We also define the category $\mathcal{O}_{K} / k^{\text {indrat }}$ and the relative fppf site $\operatorname{Spec} \mathcal{O}_{K \text {,fppf }} / k_{\text {et }}^{\text {indrat }}$ of $\mathcal{O}_{K}$ over $k$ in a similar way, using $\mathbf{O}_{K}$ instead of $\mathbf{K}$. Its cohomology theory is similarly described.

The functors

$$
\begin{aligned}
k^{\text {indrat }} & \rightarrow \mathcal{O}_{K} / k^{\text {indrat }}, \quad k^{\prime} \mapsto\left(\mathbf{O}_{K}\left(k^{\prime}\right), k^{\prime}\right), \\
\mathcal{O}_{K} / k^{\text {indrat }} & \rightarrow K / k^{\text {indrat }}, \quad\left(S, k_{S}\right) \mapsto\left(S \otimes_{\mathcal{O}_{K}} K, k_{S}\right)
\end{aligned}
$$

and their composite

$$
k^{\text {indrat }} \rightarrow K / k^{\text {indrat }}, \quad k^{\prime} \mapsto\left(\mathbf{K}\left(k^{\prime}\right), k^{\prime}\right),
$$

define morphisms

$$
\pi_{K / k}: \operatorname{Spec} K_{\mathrm{fppf}} / k_{\mathrm{et}}^{\text {indrat }} \stackrel{j}{\hookrightarrow} \operatorname{Spec} \mathcal{O}_{K, \text { fppf }} / k_{\mathrm{et}}^{\text {indrat }} \stackrel{\pi_{\mathcal{O}_{K} / k}}{\longrightarrow} \operatorname{Spec} k_{\mathrm{et}}^{\text {indrat }}
$$

of sites (see [Suz13, Def. 2.4.2] for $\pi_{K / k}$ ). We call $\pi_{K / k}$ (resp. $\pi_{\mathcal{O}_{K} / k}$ ) the fppf structure morphism of $K$ (resp. $\mathcal{O}_{K}$ ) over $k$. We denote

$$
\begin{gathered}
\boldsymbol{\Gamma}(K, \cdot)=\left(\pi_{K / k}\right)_{*}, \quad \mathbf{H}^{n}(K, \cdot)=R^{n}\left(\pi_{K / k}\right)_{*}: \operatorname{Ab}\left(K_{\mathrm{fppf}} / k_{\mathrm{et}}^{\text {indrat }}\right) \rightarrow \mathrm{Ab}\left(k_{\mathrm{et}}^{\text {indrat }}\right), \\
R \boldsymbol{\Gamma}(K, \cdot)=R\left(\pi_{K / k}\right)_{*}: D\left(K_{\mathrm{fppf}} / k_{\mathrm{et}}^{\text {indrat }}\right) \rightarrow D\left(k_{\mathrm{et}}^{\text {indrat }}\right),
\end{gathered}
$$

and similarly for $\mathcal{O}_{K}$. Again, see [KS06, Chap. 18] for the formalism of pushforward, pullback, sheaf-Hom and tensor products in unbounded derived categories and arbitrary morphisms of sites 10 For any $A \in \mathrm{Ab}\left(K_{\text {fppf }} / k_{\text {et }}^{\text {indrat }}\right)$ and $k^{\prime} \in k^{\text {indrat }}$, we have

$$
R \Gamma\left(k_{\mathrm{et}}^{\prime}, R \boldsymbol{\Gamma}(K, A)\right)=R \Gamma\left(\mathbf{K}\left(k^{\prime}\right)_{\mathrm{fppf}}, A\right),
$$

${ }^{10}$ We work with unbounded derived categories when the general theory in KS06. Chap. 18] allows us to do so without extra effort. This eliminates some unnecessary assumptions on cohomological or Tor dimensions traditionally needed for morphisms of functoriality of derived pushforward and derived cup products. However, we do not emphasize the use of unbounded derived categories. Most of the complexes we actually need turn out to be bounded after nontrivial (but essentially classical) calculations, as seen in the next subsection. Therefore, with some care, it is possible avoid unbounded derived categories in order to prove the main theorems of this paper. 
where we view $A$ as an fppf sheaf on $\mathbf{K}\left(k^{\prime}\right)$ by identifying it with the functor that sends a $\mathbf{K}\left(k^{\prime}\right)$-algebra $S$ of finite presentation to $A\left(S, k^{\prime}\right)$. If $A$ is a group scheme locally of finite type over $K$, then this identification is consistent with the identification $A\left(S, k^{\prime}\right)=A(S)$. The sheaf $\mathbf{H}^{n}(K, A)$ for any $A \in \mathrm{Ab}\left(K_{\mathrm{fppf}} / k_{\mathrm{et}}^{\text {indrat }}\right)$ is the étale sheafification of the presheaf

$$
k^{\prime} \mapsto H^{i}\left(\mathbf{K}\left(k^{\prime}\right)_{\mathrm{fppf}}, A\right),
$$

A similar description exists over $\mathcal{O}_{K}$.

The sheaf-Hom functor for Spec $K_{\mathrm{fppf}} / k_{\mathrm{et}}^{\text {indrat }}$ is simply denoted by $\mathbf{H o m}_{K}$. A morphism of fppf sheaves over $K$ (in the usual sense) induces a morphism of sheaves over Spec $K_{\mathrm{fppf}} / k_{\mathrm{et}}^{\text {indrat }}$. The derived versions are similarly denoted: $\mathbf{E x t}_{K}^{n}$ and $R \mathbf{H o m}_{K}$. There are versions $\mathbf{H o m}_{\mathcal{O}_{K}}, \mathbf{E x t}_{\mathcal{O}_{K}}^{n}$ and $R \mathbf{H o m}_{\mathcal{O}_{K}}$ over $\operatorname{Spec} \mathcal{O}_{K, \text { fppf }} / k_{\text {et }}^{\text {indrat }}$.

We define a left exact functor

$$
\begin{gathered}
\boldsymbol{\Gamma}_{x}\left(\mathcal{O}_{K}, \cdot\right): \operatorname{Ab}\left(\mathcal{O}_{K, \text { fppf }} / k_{\text {et }}^{\text {indrat }}\right) \rightarrow \operatorname{Ab}\left(k_{\text {et }}^{\text {indrat }}\right), \\
A \mapsto \operatorname{Ker}\left(\boldsymbol{\Gamma}\left(\mathcal{O}_{K}, A\right) \rightarrow \boldsymbol{\Gamma}\left(K, j^{*} A\right)\right) .
\end{gathered}
$$

This is the version of $\boldsymbol{\Gamma}\left(\mathcal{O}_{K}, \cdot\right)$ with support on the closed point $x=\operatorname{Spec} k \hookrightarrow$ Spec $\mathcal{O}_{K}$. Its derived functor is denoted by $R \boldsymbol{\Gamma}_{x}\left(\mathcal{O}_{K}, \cdot\right)$ with cohomology $\mathbf{H}_{x}^{n}\left(\mathcal{O}_{K}, \cdot\right)$. As in [Bes78, §2.2], [Mil06, III, §0, Cohomology with support on closed subscheme], this fits in the following localization triangle:

Proposition (3.3.3). We have a distinguished triangle

$$
R \boldsymbol{\Gamma}_{x}\left(\mathcal{O}_{K}, A\right) \rightarrow R \boldsymbol{\Gamma}\left(\mathcal{O}_{K}, A\right) \rightarrow R \boldsymbol{\Gamma}(K, A)
$$

in $D\left(k_{\mathrm{et}}^{\text {indrat }}\right)$ for any $A \in D\left(\mathcal{O}_{K, \mathrm{fpp}} / k_{\mathrm{et}}^{\text {indrat }}\right)$. (We frequently omit $j^{*}$ from the notation.)

Proof. The site Spec $K_{\mathrm{fppf}} / k_{\mathrm{et}}^{\text {indrat }}$ is the localization [AGV72, III, §5] of the site $\operatorname{Spec} \mathcal{O}_{K \text {,fppf }} / k_{\text {et }}^{\text {indrat }}$ at the object $(K, k)$. Hence the functor $j^{*}$ admits an exact left adjoint functor $j$ ! by AGV72, IV, Prop. 11.3.1]. The sheaf $\mathbb{Z}_{K}:=j ! \mathbb{Z}$ is represented by the usual étale $\mathcal{O}_{K}$-scheme of extension by zero of $\mathbb{Z}$, i.e. Spec $\mathcal{O}_{K} \sqcup$ $\bigsqcup_{n \in \mathbb{Z} \backslash\{0\}} \operatorname{Spec} K$. Recall from [Mil06, III, §0, Lem. 0.2] that there is an exact sequence

$$
0 \rightarrow \mathbb{Z}_{K} \rightarrow \mathbb{Z} \rightarrow \mathbb{Z}_{x} \rightarrow 0
$$

of étale group schemes over $\mathcal{O}_{K}$, where $\mathbb{Z}_{x}$ is supported on the closed point $x$ with special fiber $\mathbb{Z}$, i.e. the non-separated scheme obtained by glueing infinitely many copies of $\operatorname{Spec} \mathcal{O}_{K}$ by the common open subscheme Spec $K$. For any $A \in$ $\operatorname{Ab}\left(\mathcal{O}_{K, \text { fppf }} / k_{\text {et }}^{\text {indrat }}\right)$, the induced exact sequence

$0 \rightarrow \boldsymbol{\Gamma}\left(\mathcal{O}_{K}, \operatorname{Hom}_{\mathcal{O}_{K}}\left(\mathbb{Z}_{x}, A\right)\right) \rightarrow \boldsymbol{\Gamma}\left(\mathcal{O}_{K}, \operatorname{Hom}_{\mathcal{O}_{K}}(\mathbb{Z}, A)\right) \rightarrow \boldsymbol{\Gamma}\left(\mathcal{O}_{K}, \operatorname{Hom}_{\mathcal{O}_{K}}\left(\mathbb{Z}_{K}, A\right)\right)$

can be identified with

$$
0 \rightarrow \boldsymbol{\Gamma}_{x}\left(\mathcal{O}_{K}, A\right) \rightarrow \boldsymbol{\Gamma}\left(\mathcal{O}_{K}, A\right) \rightarrow \boldsymbol{\Gamma}\left(K, j^{*} A\right) .
$$

This extends to term-wise exact sequences of complexes when $A$ is a complex in $\operatorname{Ab}\left(\mathcal{O}_{K, \text { fppf }} / k^{\text {indrat }}\right)$.

We want to pass to the derived category. Let $A \stackrel{\sim}{\rightarrow} I$ be a quasi-isomorphism to a K-injective complex (called a homotopically injective complex in KS06, Def. 14.1.4 (i)]; for the existence of such $I$, see [KS06, Thm. 14.3.1 (ii)]). The existence of the exact left adjoint $j$ ! of $j^{*}$ implies that $j^{*}$ preserves K-injectives by the definition 
of K-injectivity. Since K-injectives calculate any right derived functors by KS06, Thm. 14.3.1 (vi)], the terms in the sequence of complexes

$$
0 \rightarrow \boldsymbol{\Gamma}_{x}\left(\mathcal{O}_{K}, I\right) \rightarrow \boldsymbol{\Gamma}\left(\mathcal{O}_{K}, I\right) \rightarrow \boldsymbol{\Gamma}\left(K, j^{*} I\right)
$$

represents

$$
R \boldsymbol{\Gamma}_{x}\left(\mathcal{O}_{K}, A\right), \quad R \boldsymbol{\Gamma}\left(\mathcal{O}_{K}, A\right), \quad R \boldsymbol{\Gamma}\left(K, j^{*} A\right),
$$

respectively.

On the other hand, the object $R \operatorname{Hom}_{\mathcal{O}_{K}}(B, A)$ for any complex $B$ can be represented by the total complex $\mathbf{H o m}_{\mathcal{O}_{K}}(B, I)$ of the sheaf-Hom double complex by KS06, Prop. 18.4.5]. This complex is K-limp in the sense of [Spa88, Def. 5.11 (a)] by Spa88, Prop. 5.14 and $\S 5.12] 11$ Hence its derived pushforward $R \boldsymbol{\Gamma}\left(\mathcal{O}_{K}, \cdot\right)$ can be calculated by applying $\boldsymbol{\Gamma}\left(\mathcal{O}_{K}, \cdot\right)$ term-wise by Spa88, Prop. 6.7 (a)] (generalized to sites). Therefore the sequence of complexes

$0 \rightarrow \boldsymbol{\Gamma}\left(\mathcal{O}_{K}, \operatorname{Hom}_{\mathcal{O}_{K}}\left(\mathbb{Z}_{x}, I\right)\right) \rightarrow \boldsymbol{\Gamma}\left(\mathcal{O}_{K}, \operatorname{Hom}_{\mathcal{O}_{K}}(\mathbb{Z}, I)\right) \rightarrow \boldsymbol{\Gamma}\left(\mathcal{O}_{K}, \operatorname{Hom}_{\mathcal{O}_{K}}\left(\mathbb{Z}_{K}, I\right)\right)$

represents the distinguished triangle

$$
\begin{aligned}
R \boldsymbol{\Gamma}\left(\mathcal{O}_{K}, R \operatorname{Hom}_{\mathcal{O}_{K}}\left(\mathbb{Z}_{x}, A\right)\right) & \rightarrow R \boldsymbol{\Gamma}\left(\mathcal{O}_{K}, R \operatorname{Hom}_{\mathcal{O}_{K}}(\mathbb{Z}, A)\right) \\
& \rightarrow R \boldsymbol{\Gamma}\left(\mathcal{O}_{K}, R \operatorname{Hom}_{\mathcal{O}_{K}}\left(\mathbb{Z}_{K}, A\right)\right)
\end{aligned}
$$

coming from $0 \rightarrow \mathbb{Z}_{K} \rightarrow \mathbb{Z} \rightarrow \mathbb{Z}_{x} \rightarrow 0$.

The above identification and distinguished triangle yield the required distinguished triangle.

Relative fppf sites have étale counterparts ([Suz13, Introduction]). The category $K_{\text {et }} / k^{\text {indrat }}$ is the full subcategory of $K / k^{\text {indrat }}$ consisting of objects $\left(L, k_{L}\right)$ with $L$ étale over $\mathbf{K}\left(k_{L}\right)$. An étale morphism $\left(L, k_{L}\right) \rightarrow\left(L^{\prime}, k_{L^{\prime}}\right)$ is a morphism with $k_{L} \rightarrow k_{L^{\prime}}$ (hence also $L \rightarrow L^{\prime}$ this case) is étale. This defines étale coverings in the category $K_{\mathrm{et}} / k^{\text {indrat }}$ and hence the relative étale site Spec $K_{\mathrm{et}} / k_{\mathrm{et}}^{\text {indrat }}$ of $K$ over $k$. The relative étale site $\operatorname{Spec} \mathcal{O}_{K \text {,et }} / k_{\text {et }}^{\text {indrat }}$ of $\mathcal{O}_{K}$ over $k$ is defined similarly. We have the étale versions

$$
\operatorname{Spec} K_{\mathrm{et}} / k_{\mathrm{et}}^{\text {indrat }} \hookrightarrow \operatorname{Spec} \mathcal{O}_{K, \mathrm{et}} / k_{\mathrm{et}}^{\text {indrat }} \rightarrow \operatorname{Spec} k_{\mathrm{et}}^{\text {indrat }} .
$$

of the structure morphisms. The notation for their pushforward functors are $R \boldsymbol{\Gamma}\left(K_{\mathrm{et}}, \cdot\right)$ and $R \boldsymbol{\Gamma}\left(\mathcal{O}_{K \text {,et }}, \cdot\right)$. We mostly consider fppf cohomology for $K$ and $\mathcal{O}_{K}$, so when there is no subscript, it means fppf cohomology.

Recall from [Suz13, Prop. 2.4.3] that we have a natural morphism

$$
R \boldsymbol{\Gamma}\left(K, R \mathbf{H o m}_{K}(A, B)\right) \rightarrow R \mathbf{H o m}_{k_{\mathrm{et}}^{\text {indrat }}}(R \boldsymbol{\Gamma}(K, A), R \boldsymbol{\Gamma}(K, B))
$$

of functoriality of $R \boldsymbol{\Gamma}$ in $D\left(k_{\mathrm{et}}^{\text {indrat }}\right)$ for any $A, B \in D\left(K_{\mathrm{fppf}} / k_{\mathrm{et}}^{\text {indrat }}\right)$. Here $R \mathbf{H o m}_{K}$ is the derived sheaf-Hom for the site Spec $K_{\mathrm{fppf}} / k_{\mathrm{et}}^{\text {indrat }}$. As we saw in loc.cit. the morphism of functoriality is equivalent to the cup-product pairing

$$
R \boldsymbol{\Gamma}(K, A) \otimes_{k}^{L} R \boldsymbol{\Gamma}(K, C) \rightarrow R \boldsymbol{\Gamma}\left(K, A \otimes_{K}^{L} C\right)
$$

11 The setting in Spa88 is over topological spaces, but can be generalized to sites in the style of [KS06 Chap. 18]. As a quick definition (cf. SSpa88, Cor. 5.17]), we say that a complex of sheaves of abelian groups $C$ on a site $S$ is K-limp if the natural morphism $\Gamma(X, C) \rightarrow R \Gamma(X, C)$ in $D(S)$ is an isomorphism for any object $X$ of $S$, where $\Gamma(X, C)$ is the complex $C$ with $\Gamma(X, \cdot)$ applied term-wise. A bounded below complex of acyclic sheaves (see the paragraph before (2.1.2) is K-limp. 
(where $\otimes_{k}^{L}$ and $\otimes_{K}^{L}$ denote the derived tensor products over Spec $k_{\mathrm{et}}^{\text {indrat }}$ and $\operatorname{Spec} K_{\mathrm{fppf}} / k_{\mathrm{et}}^{\text {indrat }}$, respectively) by the derived tensor-hom adjunction [KS06, Thm. 18.6.4 (vii)] via the change of variables $R \mathbf{H o m}_{K}(A, B) \rightsquigarrow C$ and $A \otimes_{K}^{L} C \rightsquigarrow B$.

We need a version for $R \boldsymbol{\Gamma}_{x}$. The proof of its existence is slightly different from [Suz13, Prop. 2.4.3], so we prove it here.

Proposition (3.3.4). The functoriality of $R \boldsymbol{\Gamma}_{x}$ induces natural morphisms

$$
\begin{aligned}
& R \boldsymbol{\Gamma}\left(\mathcal{O}_{K}, R \operatorname{Hom}_{\mathcal{O}_{K}}(A, B)\right) \rightarrow R \mathbf{H o m}_{k_{\mathrm{et}}^{\text {indrat }}}\left(R \boldsymbol{\Gamma}_{x}\left(\mathcal{O}_{K}, A\right), R \boldsymbol{\Gamma}_{x}\left(\mathcal{O}_{K}, B\right)\right), \\
& R \boldsymbol{\Gamma}_{x}\left(\mathcal{O}_{K}, R \boldsymbol{H o m}_{\mathcal{O}_{K}}(A, B)\right) \rightarrow R \mathbf{H o m}_{k_{\mathrm{et}}^{\text {indrat }}}\left(R \boldsymbol{\Gamma}\left(\mathcal{O}_{K}, A\right), R \boldsymbol{\Gamma}_{x}\left(\mathcal{O}_{K}, B\right)\right),
\end{aligned}
$$

for any $A, B \in D\left(\mathcal{O}_{K, \text { fppf }} / k_{\mathrm{et}}^{\text {indrat }}\right)$, where $R \mathbf{H o m}_{\mathcal{O}_{K}}$ denotes derived sheaf-Hom for the site $\operatorname{Spec} \mathcal{O}_{K, \text { fppf }} / k_{\text {et }}^{\text {indrat }}$.

Proof. For (3.3.5), let $A \stackrel{\sim}{\rightarrow} I$ and $B \stackrel{\sim}{\rightarrow} J$ be quasi-isomorphisms to K-injective complexes. As we saw in the proof of (3.3.3), the object $R \boldsymbol{\Gamma}\left(\mathcal{O}_{K}, R \operatorname{Hom}_{\mathcal{O}_{K}}(A, B)\right)$ is represented by $\boldsymbol{\Gamma}\left(\mathcal{O}_{K}, \operatorname{Hom}_{\mathcal{O}_{K}}(I, J)\right)$. The functoriality of $\boldsymbol{\Gamma}_{x}$ induces a natural morphism

$$
\boldsymbol{\Gamma}\left(\mathcal{O}_{K}, \operatorname{Hom}_{\mathcal{O}_{K}}(I, J)\right) \rightarrow \operatorname{Hom}_{k_{\mathrm{et}}^{\text {indrat }}}\left(\boldsymbol{\Gamma}_{x}\left(\mathcal{O}_{K}, I\right), \boldsymbol{\Gamma}_{x}\left(\mathcal{O}_{K}, J\right)\right)
$$

of double complexes in $\mathrm{Ab}\left(k_{\mathrm{et}}^{\text {indrat }}\right)$. In $D\left(k_{\mathrm{et}}^{\text {indrat }}\right)$, there is a natural morphism from the right-hand side to

$R \mathbf{H o m}_{k_{\mathrm{et}}^{\text {indrat }}}\left(\boldsymbol{\Gamma}_{x}\left(\mathcal{O}_{K}, I\right), \boldsymbol{\Gamma}_{x}\left(\mathcal{O}_{K}, J\right)\right)=R \mathbf{H o m}_{k_{\mathrm{et}}^{\text {indrat }}}\left(R \boldsymbol{\Gamma}_{x}\left(\mathcal{O}_{K}, A\right), R \boldsymbol{\Gamma}_{x}\left(\mathcal{O}_{K}, B\right)\right)$.

By composing, we obtain the required morphism.

For (3.3.6), we have

$$
\begin{aligned}
R \boldsymbol{\Gamma}\left(\mathcal{O}_{K}, A\right) & \rightarrow R \boldsymbol{\Gamma}\left(\mathcal{O}_{K}, R \mathbf{H o m}_{k_{\mathrm{et}}^{\text {indrat }}}\left(R \mathbf{H o m}_{k_{\mathrm{et}}^{\text {indrat }}}(A, B), B\right)\right) \\
& \rightarrow R \mathbf{H o m}_{k_{\mathrm{et}}^{\text {indrat }}}\left(R \boldsymbol{\Gamma}_{x}\left(\mathcal{O}_{K}, R \mathbf{H o m}_{k_{\mathrm{et}}^{\text {indrat }}}(A, B)\right), R \boldsymbol{\Gamma}_{x}\left(\mathcal{O}_{K}, B\right)\right),
\end{aligned}
$$

where the first morphism is the natural evaluation morphism and the second morphism is (3.3.5). In general, morphisms of the form $C \rightarrow R \operatorname{Hom}_{k_{\mathrm{et}}^{\text {indrat }}}(D, E)$ and $D \rightarrow R \mathbf{H o m}_{k_{\text {et }}^{\text {indrat }}}(C, E)$ are both equivalent to $C \otimes^{L} D \rightarrow E$ by the derived tensor-hom adjunction [KS06, Thm. 18.6.4 (vii)]. Hence the above yields the desired morphism.

These morphisms of functoriality are compatible in the following sense.

Proposition (3.3.7). Let $A, B \in D\left(\mathcal{O}_{K, \mathrm{fppf}} / k_{\mathrm{et}}^{\text {indrat }}\right)$. To simplify the notation, we denote

$$
\begin{gathered}
{[\cdot, \cdot]_{\mathcal{O}_{K}}=R \mathbf{H o m}_{\mathcal{O}_{K}}, \quad[\cdot, \cdot]_{K}=R \mathbf{H o m}_{K}, \quad[\cdot, \cdot]_{k}=R \mathbf{H o m}_{k_{\mathrm{et}}} \operatorname{Hom}_{\text {drat }},} \\
R \boldsymbol{\Gamma}_{x}=R \boldsymbol{\Gamma}_{x}\left(\mathcal{O}_{K}, \cdot\right), \quad R \boldsymbol{\Gamma}_{\mathcal{O}_{K}}=R \boldsymbol{\Gamma}\left(\mathcal{O}_{K}, \cdot\right), \quad R \boldsymbol{\Gamma}_{K}=R \boldsymbol{\Gamma}(K, \cdot) .
\end{gathered}
$$

Then we have a morphism of distinguished triangles

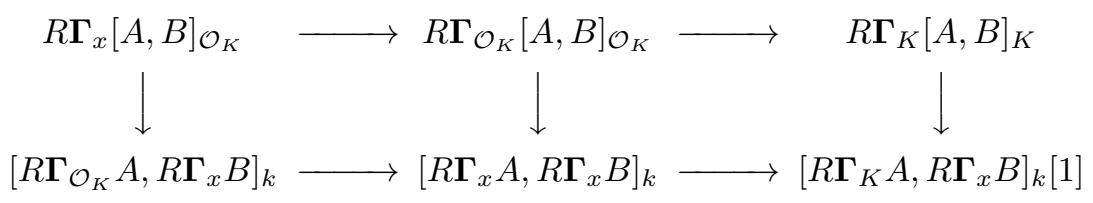

in $D\left(k_{\mathrm{et}}^{\text {indrat }}\right)$, where the horizontal triangles are the localization triangles in (3.3.3), the left two vertical morphisms are the morphisms of functoriality of $R \boldsymbol{\Gamma}_{x}$, and the 
right vertical morphism is the morphisms of functoriality of $R \boldsymbol{\Gamma}_{K}$ composed with the connecting morphism $R \boldsymbol{\Gamma}_{K} B \rightarrow R \boldsymbol{\Gamma}_{x} B[1]$ of the localization triangle.

Proof. For complexes $C, D$ in $\operatorname{Ab}\left(\mathcal{O}_{K \text {,fppf }} / k_{\mathrm{et}}^{\text {indrat }}\right)$, we denote the total complex of the sheaf-Hom double complex $\operatorname{Hom}_{\mathcal{O}_{K}}(C, D)$ by $[C, D]_{\mathcal{O}_{K}}^{c}$. Note that there is a natural morphism $[C, D]_{\mathcal{O}_{K}}^{c} \rightarrow[C, D]_{\mathcal{O}_{K}}$ in $D\left(k_{\text {et }}^{\text {indrat }}\right)$. We use the notation $[\cdot, \cdot]_{K}^{c},[\cdot, \cdot]_{k}^{c}$ similarly. We denote by $\boldsymbol{\Gamma}_{\mathcal{O}_{K}} C$ the complex $\boldsymbol{\Gamma}\left(\mathcal{O}_{K}, C\right)$, where $\boldsymbol{\Gamma}\left(\mathcal{O}_{K}, \cdot\right)$ is applied term-wise. We use the notation $\boldsymbol{\Gamma}_{K}$ similarly. We denote the mapping fiber of the morphism $\boldsymbol{\Gamma}_{\mathcal{O}_{K}} C \rightarrow \boldsymbol{\Gamma}_{K} C$ of complexes in $\mathrm{Ab}\left(k_{\mathrm{et}}^{\text {indrat }}\right)$ by $\boldsymbol{\Gamma}_{x}^{c} C$.

Let $A \stackrel{\sim}{\rightarrow} I$ and $B \stackrel{\sim}{\rightarrow} J$ be $\mathrm{K}$-injective replacements. Then $[A, B]_{\mathcal{O}_{K}}$ and $[A, B]_{K}$ can be represented by $[I, J]_{\mathcal{O}_{K}}^{c}$ and $[I, J]_{K}^{c}$, respectively. Hence $R \boldsymbol{\Gamma}_{\mathcal{O}_{K}}[A, B]_{\mathcal{O}_{K}}$, $R \boldsymbol{\Gamma}_{K}[A, B]_{K}$ and therefore $R \boldsymbol{\Gamma}_{x}[A, B]_{\mathcal{O}_{K}}$ can be represented by $\boldsymbol{\Gamma}_{\mathcal{O}_{K}}[I, J]_{\mathcal{O}_{K}}^{c}, \boldsymbol{\Gamma}_{K}[I, J]_{K}^{c}$ and $\boldsymbol{\Gamma}_{x}^{c}[I, J]_{\mathcal{O}_{K}}^{c}$, respectively. Hence, if we show that the diagram

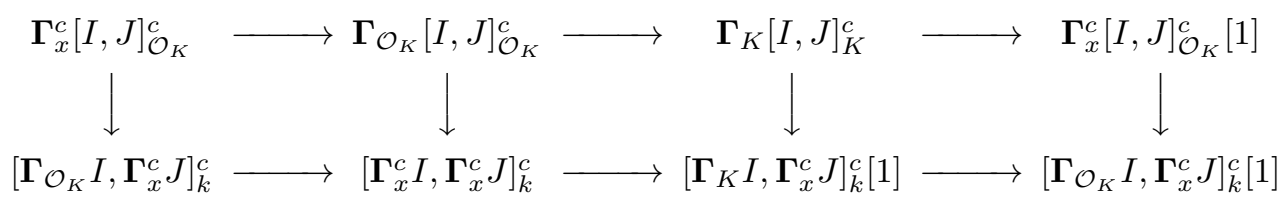

of complexes in $\operatorname{Ab}\left(k_{\text {et }}^{\text {indrat }}\right)$ is commutative up to homotopy, then we get the result by passing to the derived category and using the morphism $[\cdot, \cdot]_{k}^{c} \rightarrow[\cdot, \cdot]_{k}$. On each square, it is routine to check that the square is commutative, or to construct a homotopy up to which the square is commutative.

Now we pass to the pro-étale topology. Let $P: \operatorname{Spec} k_{\text {proet }}^{\text {indrat }} \rightarrow$ Spec $k_{\text {et }}^{\text {indrat }}$ be the morphism defined by the identity. The pullback $P^{*}$ is the pro-étale sheafification functor. We denote the composite functor $P^{*} R \boldsymbol{\Gamma}(K, \cdot)$ by $R \tilde{\boldsymbol{\Gamma}}(K, \cdot) 12$ If $A$ is an object of $\mathrm{Ab}\left(K_{\mathrm{fppf}} / k_{\mathrm{et}}^{\text {indrat }}\right)$ such that $R \boldsymbol{\Gamma}(K, A)$ is $\mathrm{P}$-acyclic, then we have

$$
R \Gamma\left(k_{\text {proet }}^{\prime}, R \tilde{\boldsymbol{\Gamma}}(K, A)\right)=R \Gamma\left(\mathbf{K}\left(k^{\prime}\right), A\right)
$$

for any $k^{\prime} \in k^{\text {indrat }}$, the sheaf $\tilde{\mathbf{H}}^{n}(K, A)$ is the étale (not pro-étale) sheafification of the presheaf

$$
k^{\prime} \mapsto H^{n}\left(\mathbf{K}\left(k^{\prime}\right), A\right),
$$

and we have

$$
\Gamma\left(k^{\prime}, \tilde{\mathbf{H}}^{n}(K, A)\right)=H^{n}\left(\mathbf{K}\left(k^{\prime}\right), A\right)
$$

for any algebraically closed $k^{\prime} \in k^{\text {indrat }}$. With this in mind, if $R \boldsymbol{\Gamma}(K, A)$ for $A \in D^{+}\left(K_{\mathrm{fppf}} / k_{\mathrm{et}}^{\text {indrat }}\right)$ is $\mathrm{P}$-acyclic, then we simply write $R \tilde{\boldsymbol{\Gamma}}(K, A)=R \boldsymbol{\Gamma}(K, A)$ following the convention made in $\$ 2.4$. Similar notation and convention will be applied to $R \boldsymbol{\Gamma}\left(\mathcal{O}_{K}, \cdot\right)$ and $R \boldsymbol{\Gamma}_{x}\left(\mathcal{O}_{K}, \cdot\right)$.

Proposition (3.3.8). There is a localization distinguished triangle

$$
R \tilde{\boldsymbol{\Gamma}}_{x}\left(\mathcal{O}_{K}, A\right) \rightarrow R \tilde{\boldsymbol{\Gamma}}\left(\mathcal{O}_{K}, A\right) \rightarrow R \tilde{\boldsymbol{\Gamma}}(K, A)
$$

\footnotetext{
12 We cannot define this functor as a certain pushforward functor without sheafification. The functor $k^{\prime} \mapsto\left(\mathbf{K}\left(k^{\prime}\right), k^{\prime}\right)$ does not define a continuous map Spec $K_{\mathrm{fppf}} / k_{\mathrm{et}}^{\text {indrat }} \rightarrow \operatorname{Spec} k_{\text {proet }}^{\text {indrat }}$, and it is not clear whether there is a nice definition of the fppf site of $K$ relative to Spec $k_{\text {proet }}^{\text {indrat }}$. For a faithfully flat ind-étale morphism $k^{\prime} \rightarrow k^{\prime \prime}$ in $k^{\text {indrat }}$, the corresponding morphism $\mathbf{O}_{K}\left(k^{\prime}\right) \rightarrow$ $\mathbf{O}_{K}\left(k^{\prime \prime}\right)$ is not finitely presented, not even ind-étale, unless $k^{\prime} \rightarrow k^{\prime \prime}$ is étale.
} 
in $D\left(k_{\text {proet }}^{\text {indrat }}\right)$ for $A \in D\left(\mathcal{O}_{K, \text { fppf }} / k_{\text {et }}^{\text {indrat }}\right)$. The functoriality of $R \tilde{\boldsymbol{\Gamma}}$ and $R \tilde{\boldsymbol{\Gamma}}_{x}$ induce morphisms

$$
R \tilde{\boldsymbol{\Gamma}}\left(K, R \mathbf{H o m}_{K}(A, B)\right) \rightarrow R \mathbf{H o m}_{k_{\text {proet }}^{\text {indrat }}}(R \tilde{\boldsymbol{\Gamma}}(K, A), R \tilde{\boldsymbol{\Gamma}}(K, B))
$$

in $D\left(k_{\text {proet }}^{\text {indrat }}\right)$ for $A, B \in D\left(K_{\text {fppf }} / k_{\text {et }}^{\text {indrat }}\right)$ and

$$
\begin{aligned}
& R \tilde{\boldsymbol{\Gamma}}\left(\mathcal{O}_{K}, R \mathbf{H o m}_{\mathcal{O}_{K}}(A, B)\right) \rightarrow R \mathbf{H o m}_{k_{\text {proet }}^{\text {indrat }}}\left(R \tilde{\boldsymbol{\Gamma}}_{x}\left(\mathcal{O}_{K}, A\right), R \tilde{\boldsymbol{\Gamma}}_{x}\left(\mathcal{O}_{K}, B\right)\right), \\
& R \tilde{\boldsymbol{\Gamma}}_{x}\left(\mathcal{O}_{K}, R \operatorname{Hom}_{\mathcal{O}_{K}}(A, B)\right) \rightarrow R \mathbf{H o m}_{k_{\text {proet }}^{\text {indrat }}}\left(R \tilde{\boldsymbol{\Gamma}}\left(\mathcal{O}_{K}, A\right), R \tilde{\boldsymbol{\Gamma}}_{x}\left(\mathcal{O}_{K}, B\right)\right),
\end{aligned}
$$

in $D\left(k_{\text {proet }}^{\text {indrat }}\right)$ for $A, B \in D\left(\mathcal{O}_{K, \text { fppf }} / k_{\text {et }}^{\text {indrat }}\right)$. With a similar set of notation to (3.3.7), we have a morphism of distinguished triangles

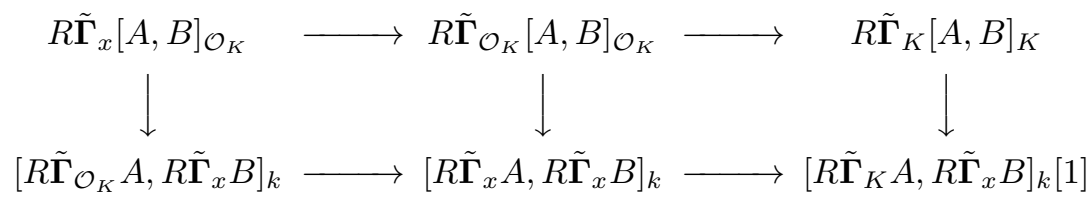

in $D\left(k_{\text {proet }}^{\text {indrat }}\right)$ for $A, B \in D\left(\mathcal{O}_{K, \text { fppf }} / k_{\text {et }}^{\text {indrat }}\right)$ (with $[\cdot, \cdot]_{k}$ this times being $\left.R \mathbf{H o m}_{k_{\text {proet }}^{\text {indrat }}}\right)$, where the horizontal triangles are localization triangles and the vertical morphisms are the functoriality morphisms together with the connecting morphism $R \tilde{\boldsymbol{\Gamma}}_{K} B \rightarrow$ $R \tilde{\boldsymbol{\Gamma}}_{x} B[1]$ on the right lower term.

Proof. We only prove the existence of the functoriality morphism of $R \tilde{\boldsymbol{\Gamma}}$. The others are treated similarly. By adjunction, we have

$$
\begin{aligned}
R \boldsymbol{\Gamma}\left(K, R \mathbf{H o m}_{K}(A, B)\right) & \rightarrow R \mathbf{H o m}_{k_{\mathrm{et}}^{\text {indrat }}}(R \boldsymbol{\Gamma}(K, A), R \boldsymbol{\Gamma}(K, B)) \\
& \rightarrow R \mathbf{H o m}_{k_{\mathrm{et}}^{\text {indrat }}}\left(R \boldsymbol{\Gamma}(K, A), R P_{*} P^{*} R \boldsymbol{\Gamma}(K, B)\right) \\
& =R P_{*} R \mathbf{H o m}_{k_{\text {proet }}^{\text {indrat }}}\left(P^{*} R \boldsymbol{\Gamma}(K, A), P^{*} R \boldsymbol{\Gamma}(K, B)\right),
\end{aligned}
$$

in $D\left(k_{\text {et }}^{\text {indrat }}\right)$. Adjoining again gives the result.

3.4. Cohomology as sheaves on the residue field. We compute $R \boldsymbol{\Gamma}$ of several group schemes over $K$ and $\mathcal{O}_{K}$. In most cases below, we obtain P-acyclic indproalgebraic groups. In some cases, the groups are in $\mathrm{PAlg} \mathrm{uc}_{\mathrm{uc}} / k$ or $\mathrm{IAlg}_{\mathrm{uc}} / k$ (having unipotent connected part), so that they are Serre reflexive. In this subsection, all sheaves over $k$, their exact sequences and distinguished triangles are considered in $\mathrm{Ab}\left(k_{\text {et }}^{\text {indrat }}\right), D\left(k_{\text {et }}^{\text {indrat }}\right)$ unless the pro-étale topology is explicitly mentioned. As soon as objects are proved to be P-acyclic, we can regard them as objects on Spec $k_{\text {proet }}^{\text {indrat }}$ without losing any information and apply the results of the previous section.

Proposition (3.4.1). The pushforward functor $\mathrm{Ab}\left(\mathcal{O}_{K, \text { et }} / k_{\text {et }}^{\text {indrat }}\right) \rightarrow \mathrm{Ab}\left(k_{\text {et }}^{\text {indrat }}\right)$ for the morphism $\operatorname{Spec} \mathcal{O}_{K, \text { et }} / k_{\text {et }}^{\text {indrat }} \rightarrow \operatorname{Spec} k_{\text {et }}^{\text {indrat }}$ is exact. Hence $\mathbf{H}^{n}\left(\mathcal{O}_{K, \text { et }}, \cdot\right)=$ 0 as functors for all $n \geq 1$.

Proof. We need to show that an étale covering $\left(S, k_{S}\right)$ of an object of the form $\left(\mathbf{O}_{K}\left(k^{\prime}\right), k^{\prime}\right)$ with $k^{\prime} \in k^{\text {indrat }}$ can be refined by a covering of the form $\left(\mathbf{O}_{K}\left(k^{\prime \prime}\right), k^{\prime \prime}\right)$ with $k^{\prime \prime}$ faithfully flat étale over $k^{\prime}$. By the $\mathbf{O}_{K}\left(k^{\prime}\right)$-version of [Suz13, Lem. 2.5.3], the étale covering $S$ of $\mathbf{O}_{K}\left(k^{\prime}\right)$ can be refined by a covering of the form $\mathcal{O}^{\prime \prime} \otimes_{\mathbf{O}_{K}^{\mathrm{fp}}\left(k^{\prime}\right)}$ $\mathbf{O}_{K}\left(k^{\prime}\right)$, where $\mathcal{O}^{\prime \prime}$ is a faithfully flat étale $\mathbf{O}_{K}^{\mathrm{fp}}\left(k^{\prime}\right)$-algebra. We can write $\mathcal{O}^{\prime \prime}=$ $\mathcal{O}_{0}^{\prime \prime} \otimes_{\mathbf{O}_{K}\left(k_{0}^{\prime}\right)} \mathbf{O}_{K}^{\mathrm{fp}}\left(k^{\prime}\right)$ with $k_{0}^{\prime}$ a rational $k$-subalgebra of $k^{\prime}$ and $\mathcal{O}_{0}^{\prime \prime}$ a faithfully flat étale 
$\mathbf{O}_{K}\left(k_{0}^{\prime}\right)$-algebra. Since $\mathbf{O}_{K}\left(k_{0}^{\prime}\right)$ is a finite product of complete discrete valuation rings with residue ring $k_{0}^{\prime}$, we may assume (by refining) that $\mathcal{O}_{0}^{\prime \prime}$ is finite over $\mathbf{O}_{K}\left(k_{0}^{\prime}\right)$ and hence written as $\mathbf{O}_{K}\left(k_{0}^{\prime \prime}\right)$ with $k_{0}^{\prime \prime}$ faithfully flat étale over $k_{0}^{\prime}$. Then we have $\mathcal{O}^{\prime \prime}=\mathbf{O}_{K}^{\mathrm{fp}}\left(k^{\prime \prime}\right)$ with $k^{\prime \prime}=k_{0}^{\prime \prime} \otimes_{k_{0}^{\prime}} k^{\prime}$ a faithfully flat étale $k^{\prime}$-algebra. Hence we have a refinement $\left(S, k_{S}\right) \rightarrow\left(\mathbf{O}_{K}\left(k^{\prime \prime}\right), k_{S}\right)$ of coverings of $\left(\mathbf{O}_{K}\left(k^{\prime}\right), k^{\prime}\right)$. The $k^{\prime}$-algebra homomorphism $k_{S} \rightarrow k^{\prime \prime}$ is étale since both $k_{S}$ and $k^{\prime \prime}$ are étale over $k^{\prime}$. Therefore the morphism $\left(\mathbf{O}_{K}\left(k^{\prime \prime}\right), k_{S}\right) \rightarrow\left(\mathbf{O}_{K}\left(k^{\prime \prime}\right), k^{\prime \prime}\right)$ is an étale covering, even though $k_{S} \rightarrow k^{\prime \prime}$ is not necessarily faithfully flat (Suz13, Prop. 2.3.3]). The composite $\left(S, k_{S}\right) \rightarrow\left(\mathbf{O}_{K}\left(k^{\prime \prime}\right), k_{S}\right) \rightarrow\left(\mathbf{O}_{K}\left(k^{\prime \prime}\right), k^{\prime \prime}\right)$ gives a desired refinement.

\section{Proposition (3.4.2).}

(a) Let $A$ be a smooth group scheme over $\mathcal{O}_{K}$ and $A_{x}$ its special fiber. Let $\boldsymbol{\Gamma}\left(\mathfrak{p}_{K}, A\right)$ be the kernel of the reduction morphism $\boldsymbol{\Gamma}\left(\mathcal{O}_{K}, A\right) \rightarrow A_{x}$. Then the sequence

$$
0 \rightarrow \boldsymbol{\Gamma}\left(\mathfrak{p}_{K}, A\right) \rightarrow \boldsymbol{\Gamma}\left(\mathcal{O}_{K}, A\right) \rightarrow A_{x} \rightarrow 0
$$

in $\mathrm{Ab}\left(k_{\mathrm{et}}^{\text {indrat }}\right)$ is exact. All the terms are P-acyclic. The group $\boldsymbol{\Gamma}\left(\mathfrak{p}_{K}, A\right)$ is connected pro-unipotent. In particular, we have $\pi_{0}\left(\boldsymbol{\Gamma}\left(\mathcal{O}_{K}, A\right)\right)=\pi_{0}\left(A_{x}\right)$, and if $A_{x}$ is of finite type, then $\boldsymbol{\Gamma}\left(\mathcal{O}_{K}, A\right) \in \mathrm{PAlg} / k$. We have $\mathbf{H}^{n}\left(\mathcal{O}_{K}, A\right)=$ 0 for all $n \geq 1$. In particular, $R \boldsymbol{\Gamma}\left(\mathcal{O}_{K}, A\right)$ is P-acyclic.

(b) Let $N$ be a finite flat group scheme over $\mathcal{O}_{K}$. Then $\boldsymbol{\Gamma}\left(\mathcal{O}_{K}, N\right)=\boldsymbol{\Gamma}(K, N) \in$ FEt $/ k, \mathbf{H}^{1}\left(\mathcal{O}_{K}, N\right)$ is connected pro-unipotent, and $\mathbf{H}^{n}\left(\mathcal{O}_{K}, N\right)=0$ for all $n \geq 2$. The complex $R \boldsymbol{\Gamma}\left(\mathcal{O}_{K}, N\right)$ is $P$-acyclic and Serre reflexive.

Proof. (国). For each $m \geq 1$, the functor $\boldsymbol{\Gamma}\left(\mathcal{O}_{K} / \mathfrak{p}_{K}^{m}, A\right): k^{\prime} \mapsto A\left(\mathbf{O}_{K} / \mathbf{p}_{K}^{m}\left(k^{\prime}\right)\right)$ is represented by the perfection of the Greenberg transform of $A$ of level $m$ (Gre61]). (3.1.3) (d) implies that $\boldsymbol{\Gamma}\left(\mathcal{O}_{K}, A\right)=\lim _{m} \boldsymbol{\Gamma}\left(\mathcal{O}_{K} / \mathfrak{p}_{K}^{m}, A\right)$. The reduction map $A\left(\mathbf{O}_{K}\left(k^{\prime}\right)\right) \rightarrow$ $A_{x}\left(k^{\prime}\right)$ is surjective for any $k^{\prime} \in k^{\text {indrat }}$ by smoothness. Hence the reduction morphism $\boldsymbol{\Gamma}\left(\mathcal{O}_{K}, A\right) \rightarrow A_{x}$ is surjective. The kernel of the surjection $\boldsymbol{\Gamma}\left(\mathcal{O}_{K} / \mathfrak{p}_{K}^{m+1}, A\right) \rightarrow$ $\boldsymbol{\Gamma}\left(\mathcal{O}_{K} / \mathfrak{p}_{K}^{m}, A\right)$ is the perfection of a vector group by Bég81, Lem. 4.1.1, 213 and the proof of [Bes78, $\$ 1.1$, Lem. 1.1 (ii)]. Therefore

$$
\boldsymbol{\Gamma}\left(\mathfrak{p}_{K}, A\right)={\underset{m}{m}}_{\lim } \operatorname{Ker}\left(\boldsymbol{\Gamma}\left(\mathcal{O}_{K} / \mathfrak{p}_{K}^{m}, A\right) \rightarrow A_{x}\right)
$$

is connected pro-unipotent and P-acyclic by (2.4.2). The quasi-algebraic group $A_{x}$ is $\mathrm{P}$-acyclic by the same proposition. Being an extension of $\mathrm{P}$-acyclics, the group $\boldsymbol{\Gamma}\left(\mathcal{O}_{K}, A\right)$ is P-acyclic. We have $\mathbf{H}^{n}\left(\mathcal{O}_{K}, A\right)=\mathbf{H}^{n}\left(\mathcal{O}_{K, \text { et }}, A\right)$ for all $n$ since $A$ is smooth and the fppf cohomology with smooth group scheme coefficients agrees with the étale cohomology by [Mil80, III, Rmk. 3.11 (b)]. We have $\mathbf{H}^{n}\left(\mathcal{O}_{K, \text { et }}, A\right)=0$ for $n \geq 1$ by the previous proposition.

(b) The functors $\boldsymbol{\Gamma}\left(\mathcal{O}_{K}, N\right): k^{\prime} \mapsto N\left(\mathbf{O}_{K}\left(k^{\prime}\right)\right)$ and $\boldsymbol{\Gamma}(K, N): k^{\prime} \mapsto N\left(\mathbf{K}\left(k^{\prime}\right)\right)$ for finite flat $N$ are represented by the same finite étale $k$-scheme by (3.2.10).

For cohomology of degree $\geq 1$, recall from [Bég81, Prop. 2.2.1] that there is an exact sequence $0 \rightarrow N \rightarrow G \rightarrow H \rightarrow 0$ of group schemes over $\mathcal{O}_{K}$ with $G, H$ smooth affine with connected fibers (more specifically, $G$ is the Weil restriction of $\mathbf{G}_{m}$ from

\footnotetext{
13 Note that this lemma by Bégueri is true only after perfection. See BGA13, Rmk. 14.22, 15.9]. In our case, it is enough to check this lemma for perfect-field-valued points only by the following reason. The sheaf $\boldsymbol{\Gamma}\left(\mathcal{O}_{K} / \mathfrak{p}_{K}^{m}, A\right)$ on Spec $k_{\text {et }}^{\text {indrat }}$ is locally of finite presentation and the kernel of the surjection mentioned here is also locally of finite presentation. Hence it is enough to treat them as sheaves on Spec $k_{\text {et }}^{\text {rat }}$. For perfect-field-valued points, the lemma is classical.
} 
the Cartier dual $N^{\mathrm{CD}}$ to $\operatorname{Spec} \mathcal{O}_{K}$ and $N \hookrightarrow G$ is the natural embedding). The long exact sequence shows that $\mathbf{H}^{n}\left(\mathcal{O}_{K}, N\right)=0$ for $n \geq 2$ and yields an exact sequence

$$
0 \rightarrow \boldsymbol{\Gamma}\left(\mathcal{O}_{K}, N\right) \rightarrow \boldsymbol{\Gamma}\left(\mathcal{O}_{K}, G\right) \rightarrow \boldsymbol{\Gamma}\left(\mathcal{O}_{K}, H\right) \rightarrow \mathbf{H}^{1}\left(\mathcal{O}_{K}, N\right) \rightarrow 0
$$

(in $\mathrm{Ab}\left(k_{\text {et }}^{\text {indrat }}\right)$ a priori). The first three terms are P-acyclic. Hence so is the fourth. Therefore the sequence is exact also in $\mathrm{Ab}\left(k_{\text {proet }}^{\text {indrat }}\right)$. Since the first term is finite and the second and third terms are connected proalgebraic, the fourth term is connected proalgebraic. Since $\mathbf{H}^{1}\left(\mathcal{O}_{K}, N\right)$ is killed by the order of $N$, it does not have semiabelian part. Hence $\mathbf{H}^{1}\left(\mathcal{O}_{K}, N\right)$ is pro-unipotent. Therefore $R \boldsymbol{\Gamma}\left(\mathcal{O}_{K}, N\right)$ is Serre reflexive by (2.4.1) (b).

\section{Proposition (3.4.3).}

(a) Let $A$ be a smooth group scheme over $K$. Then $\mathbf{H}^{n}(K, A)$ for any $n \geq$ 1 is torsion and locally of finite presentation as a functor on $k^{\text {indrat }}$. In particular, it is P-acyclic.

(b) Let $N$ be a finite flat group scheme over $K$. Then $\boldsymbol{\Gamma}(K, N) \in \mathrm{FEt} / k$, $\mathbf{H}^{1}(K, N) \in \mathrm{IPAlg}_{\mathrm{uc}} / k, \mathbf{H}^{n}(K, N)=0$ for all $n \geq 2$, and $R \boldsymbol{\Gamma}(K, N)$ is $P$-acyclic and Serre reflexive. The group $\mathbf{H}^{1}(K, N)$ is in $\mathrm{IAlg}_{\mathrm{uc}} / k$ if $N$ is étale and in $\mathrm{PAlg}_{\mathrm{uc}} / k$ if $N$ is multiplicative. If $K$ has mixed characteristic, then $\mathbf{H}^{1}(K, N) \in \operatorname{Alg}_{\mathrm{uc}} / k$.

(c) Let $Y$ be a lattice over $K$ (i.e., a finite free abelian group with a Galois action of $K)$. Then $\boldsymbol{\Gamma}(K, Y)$ is a lattice over $k, \mathbf{H}^{1}(K, Y) \in \mathrm{FEt} / k, \mathbf{H}^{2}(K, Y) \in$ $\mathrm{IAlg}_{\mathrm{uc}} / k, \mathbf{H}^{n}(K, Y)=0$ for $n \geq 3$, and $R \boldsymbol{\Gamma}(K, Y)$ is P-acyclic and Serre reflexive.

(d) Let $A$ be an abelian variety over $K$ with Néron model $\mathcal{A}$. Then $\boldsymbol{\Gamma}(K, A)=$ $\boldsymbol{\Gamma}\left(\mathcal{O}_{K}, \mathcal{A}\right) \in \mathrm{PAlg} / k$, which is described by the previous proposition, $\mathbf{H}^{1}(K, A) \in$ $\mathrm{IAlg}_{\mathrm{uc}} / k, \mathbf{H}^{n}(K, A)=0$ for all $n \geq 2$, and $R \boldsymbol{\Gamma}(K, A)$ is $P$-acyclic.

(e) Let $T$ be a torus over $K$ with Néron model $\mathcal{T}$. Then $\boldsymbol{\Gamma}(K, T)=\boldsymbol{\Gamma}\left(\mathcal{O}_{K}, \mathcal{T}\right)$ is an extension of an étale group by a $P$-acyclic proalgebraic group described by the previous proposition. We have $\mathbf{H}^{n}(K, T)=0$ for all $n \geq 1$, and $R \boldsymbol{\Gamma}(K, T)$ is P-acyclic.

Proof. (国) (3.2.8) shows that the functor $k^{\prime} \mapsto H^{n}\left(\mathbf{K}\left(k^{\prime}\right)_{\text {et }}, A\right)$ is locally of finite presentation for $n \geq 1$ and takes values in torsion groups. Hence so is its étale sheafification $\mathbf{H}^{n}(K, A)$. Therefore $\mathbf{H}^{n}(K, A)$ is torsion and $\mathrm{P}$-acyclic by (2.4.2) (a).

(b) By (3.2.10), the sheaf $\boldsymbol{\Gamma}(K, N)$ is a finite étale group over $k$ whose $\bar{k}$-points is given by $\Gamma\left(K^{\mathrm{ur}}, N\right)$. (Note that $N$ does not have to extend to $\mathcal{O}_{K}$ and hence we are not using (3.4.2) here.)

Suppose that $N$ has order prime to $p$. Then it is classical to see that $H^{n}\left(K^{\mathrm{ur}}, N\right)$ is zero for $n \geq 2$ and $H^{1}\left(K^{\mathrm{ur}}, N\right)$ is finite. These groups do not change under residue field extensions, i.e. for any algebraically closed field $k^{\prime}$ over $k$, we have $H^{n}\left(\mathbf{K}\left(k^{\prime}\right), N\right)=H^{n}\left(K^{\mathrm{ur}}, N\right)$ for any $n \geq 1$. Since $N$ is étale, the sheaf $\mathbf{H}^{n}(K, N)$ is locally of finite presentation for any $n \geq 1$ by the previous assertion. Hence it is determined by $k^{\prime}$-points for various algebraically closed fields $k^{\prime}$. Therefore $\mathbf{H}^{n}(K, N)=0$ for $n \geq 2$ and $\mathbf{H}^{1}(K, N)$ is the finite étale group given by $H^{1}\left(K^{\mathrm{ur}}, N\right)$.

Suppose that $N$ is $p$-primary. Suppose also that $K$ has equal characteristic. We first show the statements for $N=\mathbb{Z} / p \mathbb{Z}, \mu_{p}$ and $\alpha_{p}$. If $N=\mathbb{Z} / p \mathbb{Z}$, then 


$$
\begin{aligned}
& \mathbf{H}^{n}(K, \mathbb{Z} / p \mathbb{Z})=0 \text { for } n \geq 2 \text { and } \\
& \qquad \mathbf{H}^{1}(K, \mathbb{Z} / p \mathbb{Z})=\mathbf{K} / \wp(\mathbf{K}) \cong \mathbf{G}_{a}^{\oplus \mathbb{N}} \in \operatorname{IAlg}_{\mathrm{uc}} / k
\end{aligned}
$$

by the Artin-Schreier sequence $0 \rightarrow \mathbb{Z} / p \mathbb{Z} \rightarrow \mathbf{G}_{a} \stackrel{\wp}{\rightarrow} \mathbf{G}_{a} \rightarrow 0$ and that $\mathbf{H}^{n}\left(K, \mathbf{G}_{a}\right)=$ 0 for all $n \geq 1$. In particular, $\mathbf{H}^{1}(K, \mathbb{Z} / p \mathbb{Z})$ is $\mathrm{P}$-acyclic and Serre reflexive by (2.4.1) (b) and (2.4.2) (a). For $N=\mu_{p}$, the paragraph before [Suz13, Lem. 2.7.4] and the proof of the cited lemma show that $\mathbf{H}^{n}\left(K, \mu_{p}\right)=0$ for $n \geq 2$ and

$$
\mathbf{H}^{1}\left(K, \mu_{p}\right)=\mathbf{K}^{\times} /\left(\mathbf{K}^{\times}\right)^{p} \cong \mathbb{Z} / p \mathbb{Z} \times \mathbf{G}_{a}^{\mathbb{N}} \in \mathrm{PAlg}_{\mathrm{uc}} / k .
$$

In particular, $\mathbf{H}^{1}\left(K, \mu_{p}\right)$ is $\mathrm{P}$-acyclic by (2.4.2) and Serre reflexive by (2.4.1) (b). For $N=\alpha_{p}$, a calculation similar to the case of $\mathbb{Z} / p \mathbb{Z}$ shows that $\mathbf{H}^{n}\left(K, \alpha_{p}\right)=0$ for $n \geq 2$ and

$$
\mathbf{H}^{1}\left(K, \alpha_{p}\right)=\mathbf{K} / \mathbf{K}^{p} \cong \mathbf{G}_{a}^{\oplus \mathbb{N}} \times \mathbf{G}_{a}^{\mathbb{N}} \in \mathrm{IPAlg}_{\mathrm{uc}} / k .
$$

Each factor is $\mathrm{P}$-acyclic and Serre reflexive by the same propositions. Hence so is the product.

Next, if $0 \rightarrow N_{1} \rightarrow N_{2} \rightarrow N_{3} \rightarrow 0$ is an exact sequence of finite flat group schemes over $K$, and if we know that $N_{1}$ and $N_{3}$ satisfy the statements, then the long exact sequence

$$
\begin{aligned}
0 & \rightarrow \boldsymbol{\Gamma}\left(K, N_{1}\right) \rightarrow \boldsymbol{\Gamma}\left(K, N_{2}\right) \rightarrow \boldsymbol{\Gamma}\left(K, N_{3}\right) \\
\rightarrow \mathbf{H}^{1}\left(K, N_{1}\right) & \rightarrow \mathbf{H}^{1}\left(K, N_{2}\right) \rightarrow \mathbf{H}^{1}\left(K, N_{3}\right) \rightarrow 0
\end{aligned}
$$

and the finiteness of $\boldsymbol{\Gamma}\left(K, N_{i}\right)(i=1,2,3)$ implies that $N_{3}$ satisfies the statements. Therefore if $N$ has a filtration whose successive subquotients are $\mathbb{Z} / p \mathbb{Z}, \mu_{p}$ or $\alpha_{p}$, then $N$ satisfies the statements.

Now, let $N$ be any $p$-primary finite flat group scheme over $K$. Then $N$ has a filtration whose successive subquotients are étale, multiplicative or $\alpha_{p}$ by DG70, IV, $\S 3,5.8,5.9]$. Let $L$ be a finite Galois extension of $K$ that trivializes the Galois actions on the étale part and the Cartier dual of the multiplicative part. It is enough to show the statements after a finite unramified extension, so we may assume that $L / K$ is totally ramified. Let $M$ be the intermediate field of $L / K$ that corresponds to a (or the) $p$-Sylow subgroup of $\operatorname{Gal}(L / K)$. If a $p$-group acts on a non-zero $\mathbb{F}_{p}$-vector space, then it has a non-zero fixed part by Ser79, IX, Lem. 4]. Hence the base-changed group $N \times_{K} M$ over $M$ has a filtration whose successive subquotients are $\mathbb{Z} / p \mathbb{Z}, \mu_{p}$ or $\alpha_{p}$. Therefore $N \times_{K} M$ satisfies the statements, i.e. $\mathbf{H}^{1}(M, N) \in \mathrm{IPAlg} / k$ (IAlg $/ k$ if $N$ is étale, $\mathrm{PAlg} / k$ if multiplicative), $\mathbf{H}^{n}(M, N)=0$ for $n \geq 2$ and $R \boldsymbol{\Gamma}(M, N)$ is $\mathrm{P}$-acyclic and Serre reflexive. We have $R \boldsymbol{\Gamma}(M, N)=R \boldsymbol{\Gamma}\left(K, \operatorname{Res}_{M / K} N\right)$, where $\operatorname{Res}_{M / K}$ denotes the Weil restriction functor. The composite of the inclusion map $N \hookrightarrow \operatorname{Res}_{M / K} N$ and the norm map $\operatorname{Res}_{M / K} N \rightarrow N$ is the multiplication by [M:K], which is an isomorphism since $N$ is $p$-primary and $[M: K]$ is prime to $p$. Hence $N$ is a direct summand of $\operatorname{Res}_{M / K} N$. Therefore $N$ (over $K$ ) satisfies the statements.

Suppose next that $K$ has mixed characteristic. If $N=\mu_{p}$, then $\mathbf{H}^{1}\left(K, \mu_{p}\right)=$ $\mathbf{K}^{\times} /\left(\mathbf{K}^{\times}\right)^{p}$ similarly. We have $\mathbf{K}^{\times} \cong \mathbb{Z} \times \mathbf{U}_{K}$ by [Suz13, the paragraph before Prop. 2.4.4]. The group $\mathbf{U}_{K}=\mathbf{O}_{K}^{\times}=\boldsymbol{\Gamma}\left(\mathcal{O}_{K}, \mathbf{G}_{m}\right)$ is P-acyclic by (3.4.2) (可). Since $\boldsymbol{\Gamma}\left(K, \mu_{p}\right)=0$ or $\mathbb{Z} / p \mathbb{Z}$, The exact sequence

$$
0 \rightarrow \boldsymbol{\Gamma}\left(K, \mu_{p}\right) \rightarrow \mathbf{K}^{\times} \stackrel{p}{\rightarrow} \mathbf{K}^{\times} \rightarrow \mathbf{K}^{\times} /\left(\mathbf{K}^{\times}\right)^{p} \rightarrow 0
$$


shows that $\mathbf{K}^{\times} /\left(\mathbf{K}^{\times}\right)^{p}$ and $\left(\mathbf{K}^{\times}\right)^{p}$ are P-acyclic. The logarithm map shows that $\left(\mathbf{K}^{\times}\right)^{p}$ contains the group $\mathbf{U}_{K}^{m}=1+\mathbf{p}_{K}^{m}$ of $m$-th principal units for some $m$. Since $\mathbf{U}_{K} / \mathbf{U}_{K}^{m}$ is an $m$-dimensional quasi-algebraic group, its quotient $\mathbf{U}_{K} /\left(\mathbf{U}_{K}\right)^{p}=$ $\mathbf{U}_{K}^{1} /\left(\mathbf{U}_{K}^{1}\right)^{p}$ is quasi-algebraic unipotent. Hence $\mathbf{H}^{1}\left(K, \mu_{p}\right) \in \operatorname{Alg}_{\mathrm{uc}} / k$. Then the same process as the equal characteristic case shows that $\mathbf{H}^{1}(K, N) \in \mathrm{Alg}_{\mathrm{uc}} / k$ for any finite flat (hence étale) $N$ and $N$ satisfies the statements.

(IC) We have an exact sequence $0 \rightarrow Y \rightarrow Y \otimes \mathbb{Q} \rightarrow Y \otimes \mathbb{Q} / \mathbb{Z} \rightarrow 0$. We have $H^{n}\left(K^{\text {ur }}, Y \otimes \mathbb{Q}\right)=0$ for $n \geq 1$. From this, since $Y \otimes \mathbb{Q}$ is étale and hence smooth, we know that $\mathbf{H}^{n}(K, Y \otimes \mathbb{Q})=0$ for $n \geq 1$ by the same method as the second paragraph of the proof of the previous assertion. Therefore

$$
\mathbf{H}^{n}(K, Y)=\mathbf{H}^{n-1}(K, Y \otimes \mathbb{Q} / \mathbb{Z})=\underset{m}{\lim } \mathbf{H}^{n-1}(K, Y \otimes \mathbb{Z} / m \mathbb{Z})
$$

for $n \geq 2$. This sheaf is zero for $n \geq 3$ and in $\mathrm{IAlg}_{\mathrm{uc}} / k$ for $n=2$ by the previous assertion.

By (3.2.10), we know that $\boldsymbol{\Gamma}(K, Y)$ is a lattice over $k$. Let $I$ be the inertia group of a finite Galois extension of $K$ over which $Y$ becomes trivial. The same proposition shows that the exact sequence

$$
0 \rightarrow \boldsymbol{\Gamma}(K, Y) \rightarrow \boldsymbol{\Gamma}(K, Y \otimes \mathbb{Q}) \rightarrow \boldsymbol{\Gamma}(K, Y \otimes \mathbb{Q} / \mathbb{Z}) \rightarrow \mathbf{H}^{1}(K, Y) \rightarrow 0
$$

is identified with the exact sequence

$$
0 \rightarrow H^{0}(I, Y) \rightarrow H^{0}(I, Y \otimes \mathbb{Q}) \rightarrow H^{0}(I, Y \otimes \mathbb{Q} / \mathbb{Z}) \rightarrow H^{1}(I, Y) \rightarrow 0
$$

of group cohomology groups. Since $I$ is finite and $Y$ finitely generated, we know that $H^{1}(I, Y)$ is finite [Ser79, VIII, $\S 2$, Cor. 2]. Hence $\mathbf{H}^{1}(K, Y)$ is finite étale.

Therefore $R \boldsymbol{\Gamma}(K, Y)$ has Serre reflexive cohomologies by (2.4.1) (b) and hence itself is Serre reflexive.

(d) We have $\boldsymbol{\Gamma}(K, A)=\boldsymbol{\Gamma}\left(\mathcal{O}_{K}, \mathcal{A}\right)$ by (3.1.3) (ㄷ). This is in PAlg $/ k$ by the previous proposition. We know that $\mathbf{H}^{n}(K, A)$ is torsion for any $n \geq 1$ by (a) . For each $m \geq 1$, the exact sequence $0 \rightarrow A[m] \rightarrow A \rightarrow A \rightarrow 0$ yields an exact sequence

$$
0 \rightarrow \boldsymbol{\Gamma}(K, A) / m \boldsymbol{\Gamma}(K, A) \rightarrow \mathbf{H}^{1}(K, A[m]) \rightarrow \mathbf{H}^{1}(K, A)[m] \rightarrow 0 .
$$

The first term is $\mathrm{P}$-acyclic since $\boldsymbol{\Gamma}(K, A)$ and its $m$-torsion part $\boldsymbol{\Gamma}(K, A[m])$ are so. It is in $\mathrm{PAlg}_{\mathrm{uc}} / k$, since its semi-abelian part is divisible and killed by $m$, hence zero. The middle term is in IPAlg $\operatorname{uc}_{\mathrm{uc}} / k$ and P-acyclic by the finite flat case. Therefore the right term is in $\mathrm{IPAlg}_{\mathrm{uc}} / k$ and $\mathrm{P}$-acyclic. So is the filtered union $\mathbf{H}^{1}(K, A)$. We know that $\mathbf{H}^{1}(K, A)$ is locally of finite presentation by (国). Hence (3.4.4) below implies that $\mathbf{H}^{1}(K, A) \in \mathrm{IAlg}_{\mathrm{uc}} / k$. Similarly the vanishing $\mathbf{H}^{n}(K, A[m])=0$ of the finite flat case implies $\mathbf{H}^{n}(K, A)=0$ for $n \geq 2$.

(四) For $\boldsymbol{\Gamma}$, it is similar to abelian varieties (though in this case $\mathcal{T}$ is only locally of finite type). To show $\mathbf{H}^{n}(K, T)=0$ for $n \geq 1$, it is enough to see that $H^{n}\left(\mathbf{K}\left(k^{\prime}\right), T\right)=0$ for any algebraically closed field $k^{\prime}$ over $k$ by (国). This is given in [Ser79, X §7, Application].

Lemma (3.4.4). If $A \in \mathrm{IPAlg} / k$ is locally of finite presentation as a functor on $k^{\text {indrat}}$, then we have $A \in \mathrm{IAlg} / k$.

Proof. Let $A=\lim A_{\lambda}$ with $A_{\lambda} \in \mathrm{PAlg} / k$. We need to show that any morphism $B \rightarrow A$ from an object $B \in \mathrm{PAlg} / k$ factors through an object of $\mathrm{Alg} / k$. Let $B=\lim _{\longleftarrow} B_{\mu}$ with $B_{\mu} \in \mathrm{Alg} / k$. We may assume that the transition morphisms 
$B_{\mu^{\prime}} \rightarrow B_{\mu}$ are surjective. Let $\xi_{B}$ be the generic point of $B$ in the sense of [Suz13, Def. 3.2.1], namely $\xi_{B}=\lim \xi_{B_{\mu}}$ and $\xi_{B_{\mu}}$ is the disjoint union of the generic points of the irreducible components of $B_{\mu}$. As a scheme, $\xi_{B}$ is the Spec of an ind-rational $k$-algebra $k_{B}^{\prime}$. For any $C \in \mathrm{Ab}\left(k_{\text {proet }}^{\text {indrat }}\right)$, we denote $C\left(\xi_{B}\right)=C\left(k_{B}^{\prime}\right)$. We define a group homomorphism $\sigma: C\left(\xi_{B}\right) \rightarrow C\left(\xi_{B \times{ }_{k} B}\right)$ by sending a morphism $f: \xi_{B} \rightarrow C$ to the morphism $\xi_{B \times B} \rightarrow C$ given by $\left(b_{1}, b_{2}\right) \mapsto f\left(b_{1}\right)+f\left(b_{2}\right)-f\left(b_{1}+b_{2}\right)$. Then for $C \in \mathrm{PAlg} / k$, we have

$$
\operatorname{Hom}(B, C)=\operatorname{Ker}\left(C\left(\xi_{B}\right) \stackrel{\sigma}{\rightarrow} C\left(\xi_{B \times B}\right)\right),
$$

which means that a homomorphism of birational groups extends to a everywhere regular group homomorphism ([Ser88, V, §1.5, Lem. 6] plus a limit argument). Now let $C=A$. Then using the assumption, we have

$$
\begin{aligned}
\operatorname{Hom}(B, A) & =\underset{\lambda}{\lim } \operatorname{Hom}\left(B, A_{\lambda}\right) \\
& =\underset{\lambda}{\lim } \operatorname{Ker}\left(A_{\lambda}\left(\xi_{B}\right) \rightarrow A_{\lambda}\left(\xi_{B \times B}\right)\right) \\
& =\underset{\lambda, \mu}{\lim } \operatorname{Ker}\left(A_{\lambda}\left(\xi_{B_{\mu}}\right) \rightarrow A_{\lambda}\left(\xi_{B_{\mu} \times B_{\mu}}\right)\right) \\
& =\underset{\lambda, \mu}{\lim } \operatorname{Hom}\left(B_{\mu}, A_{\lambda}\right) \\
& =\underset{\mu}{\lim } \operatorname{Hom}\left(B_{\mu}, A\right) .
\end{aligned}
$$

Hence a morphism $B \rightarrow A$ factors through some $B_{\mu} \in \mathrm{Alg} / k$.

Proposition (3.4.6). Let $N$ be a finite flat group scheme over $\mathcal{O}_{K}$. Then $\mathbf{H}_{x}^{n}\left(\mathcal{O}_{K}, N\right)=$ 0 for $n \neq 2$. We have $\mathbf{H}_{x}^{2}\left(\mathcal{O}_{K}, N\right) \in \operatorname{IAlg}_{\mathrm{uc}} / k$, and $R \boldsymbol{\Gamma}_{x}\left(\mathcal{O}_{K}, N\right)$ is P-acyclic and Serre reflexive. If $K$ has mixed characteristic, then $\mathbf{H}_{x}^{2}\left(\mathcal{O}_{K}, N\right) \in \operatorname{Alg}_{\mathrm{uc}} / k$.

Proof. We have $\boldsymbol{\Gamma}\left(\mathcal{O}_{K}, N\right)=\boldsymbol{\Gamma}(K, N)$ and $\mathbf{H}^{n}\left(\mathcal{O}_{K}, N\right)=\mathbf{H}^{n}(K, N)=0$ for $n \geq 2$ by the previous two propositions. We show that the morphism $\mathbf{H}^{1}\left(\mathcal{O}_{K}, N\right) \rightarrow$ $\mathbf{H}^{1}(K, N)$ is injective. Let $k^{\prime} \in k^{\text {indrat }}$ and $X$ an fppf $N$-torsor over $\operatorname{Spec} \mathbf{O}_{K}\left(k^{\prime}\right)$. Since $N$ is finite, we have $X\left(\mathbf{O}_{K}\left(k^{\prime}\right)\right)=X\left(\mathbf{K}\left(k^{\prime}\right)\right)$ by (3.1.3) (C). Hence if $X$ maps to zero under $H^{1}\left(\mathbf{O}_{K}\left(k^{\prime}\right), N\right) \rightarrow H^{1}\left(\mathbf{K}\left(k^{\prime}\right), N\right)$, then it is zero. Therefore $H^{1}\left(\mathbf{O}_{K}\left(k^{\prime}\right), N\right) \rightarrow H^{1}\left(\mathbf{K}\left(k^{\prime}\right), N\right)$ is injective and $\mathbf{H}^{1}\left(\mathcal{O}_{K}, N\right) \rightarrow \mathbf{H}^{1}(K, N)$ is injective. Hence the localization triangle (3.3.3)

$$
R \boldsymbol{\Gamma}_{x}\left(\mathcal{O}_{K}, N\right) \rightarrow R \boldsymbol{\Gamma}\left(\mathcal{O}_{K}, N\right) \rightarrow R \boldsymbol{\Gamma}(K, N)
$$

in $D\left(k_{\text {et }}^{\text {indrat }}\right)$ reduces to an exact sequence

$$
0 \rightarrow \mathbf{H}^{1}\left(\mathcal{O}_{K}, N\right) \rightarrow \mathbf{H}^{1}(K, N) \rightarrow \mathbf{H}_{x}^{2}\left(\mathcal{O}_{K}, N\right) \rightarrow 0
$$

in $\mathrm{Ab}\left(k_{\mathrm{et}}^{\text {indrat }}\right)$. Since the first two terms are P-acyclic, Serre reflexive and in IPAlg ${ }_{\mathrm{uc}} / k$ by (3.4.2) (B) and (3.4.3) (b)), so is the third $\mathbf{H}_{x}^{2}\left(\mathcal{O}_{K}, N\right)$.

To deduce $\mathbf{H}_{x}^{2}\left(\mathcal{O}_{K}, N\right) \in \mathrm{IAlg}_{\mathrm{uc}} / k$, let $0 \rightarrow N \rightarrow G \rightarrow H \rightarrow 0$ be an exact sequence of group schemes over $\mathcal{O}_{K}$ with $G, H$ smooth affine with connected fibers, as we took in the proof of (3.4.2) (b). Since $R \boldsymbol{\Gamma}\left(\mathcal{O}_{K}, G\right)=R \boldsymbol{\Gamma}\left(\mathcal{O}_{K, \text { et }}, G\right)$ is concentrated in degree 0 by (3.4.1) and the morphism $\boldsymbol{\Gamma}\left(\mathcal{O}_{K}, G\right) \rightarrow \boldsymbol{\Gamma}(K, G)$ is injective, we know that

$$
\begin{gathered}
\boldsymbol{\Gamma}_{x}\left(\mathcal{O}_{K}, G\right)=0, \quad \mathbf{H}_{x}^{1}\left(\mathcal{O}_{K}, G\right)=\boldsymbol{\Gamma}(K, G) / \boldsymbol{\Gamma}\left(\mathcal{O}_{K}, G\right), \\
\mathbf{H}_{x}^{n}\left(\mathcal{O}_{K}, G\right)=\mathbf{H}^{n-1}(K, G), \quad n \geq 2 .
\end{gathered}
$$


These are locally of finite presentation by (3.2.8) and (3.2.9). Similarly, $\mathbf{H}_{x}^{n}\left(\mathcal{O}_{K}, H\right)$ is locally of finite presentation for any $n$. The distinguished triangle $R \boldsymbol{\Gamma}_{x}\left(\mathcal{O}_{K}, N\right) \rightarrow$ $R \boldsymbol{\Gamma}_{x}\left(\mathcal{O}_{K}, G\right) \rightarrow R \boldsymbol{\Gamma}_{x}\left(\mathcal{O}_{K}, H\right)$ then shows that $\mathbf{H}_{x}^{2}\left(\mathcal{O}_{K}, N\right)$ is locally of finite presentation. We saw above that $\mathbf{H}_{x}^{2}\left(\mathcal{O}_{K}, N\right) \in \mathrm{IPAlg} \lg _{\mathrm{uc}} / k$, Hence (3.4.4) implies that $\mathbf{H}_{x}^{2}\left(\mathcal{O}_{K}, N\right) \in \operatorname{IAlg}_{\mathrm{uc}} / k$.

If $K$ has mixed characteristic, then $\mathbf{H}^{1}(K, N) \in \operatorname{Alg}_{\mathrm{uc}} / k$ implies $\mathbf{H}^{1}\left(\mathcal{O}_{K}, N\right) \in$ $\operatorname{Alg}_{\mathrm{uc}} / k$, so $\mathbf{H}_{x}^{2}\left(\mathcal{O}_{K}, N\right) \in \operatorname{Alg}_{\mathrm{uc}} / k$.

Remark (3.4.7). Another method to compute $R \boldsymbol{\Gamma}\left(\mathcal{O}_{K}, N\right), R \boldsymbol{\Gamma}(K, N)$ and $R \boldsymbol{\Gamma}_{x}\left(\mathcal{O}_{K}, N\right)$ for finite flat $N$ over equal characteristic $K$ is to use the two exact sequences of [Mil06, III, §5] (see also \$5.2.2 of this paper). Then these cohomology complexes can be calculated by the cohomology with coefficients in vector groups in the case $N$ or the Cartier dual $N^{\mathrm{CD}}$ has height 1 . The general case follows by dévissage. This method is due to Artin-Milne [AM76] in the global situation and Bester Bes78] in the local situation.

\section{Statement of the Duality theorem}

From now on throughout the paper, all sheaves over $k$, their exact sequences and distinguished triangles are considered in $\mathrm{Ab}\left(k_{\text {proet }}^{\text {indrat }}\right), D\left(k_{\text {proet }}^{\text {indrat }}\right)$ unless otherwise noted. We denote $R \boldsymbol{\Gamma}(\cdot)=R \boldsymbol{\Gamma}(K, \cdot)$ when there is no confusion.

4.1. Formulation. We formulate the duality theorem with coefficients in abelian varieties. First, $R \boldsymbol{\Gamma}\left(\mathbf{G}_{m}\right)$ is P-acyclic by (3.4.3) (E) , so we write $R \tilde{\boldsymbol{\Gamma}}\left(\mathbf{G}_{m}\right)=$ $R \boldsymbol{\Gamma}\left(\mathbf{G}_{m}\right)$ in $D\left(k_{\text {proet }}^{\text {indrat }}\right)$. The same assertion or [Suz13, Prop. 2.4.4] shows that

$$
R \boldsymbol{\Gamma}\left(\mathbf{G}_{m}\right)=\boldsymbol{\Gamma}\left(\mathbf{G}_{m}\right)=\mathbf{K}^{\times} .
$$

Recall from the paragraph before [Suz13. Prop. 2.4.4] that there are the valuation map $\mathbf{K}^{\times} \rightarrow \mathbb{Z}$ as a morphism of sheaves and a split exact sequence

$$
0 \rightarrow \mathbf{U}_{K} \rightarrow \mathbf{K}^{\times} \rightarrow \mathbb{Z} \rightarrow 0,
$$

where $\mathbf{U}_{K}=\mathbf{O}_{K}^{\times}$. An alternative definition of this sequence and the valuation map is the exact sequence

$$
0 \rightarrow \boldsymbol{\Gamma}\left(\mathcal{O}_{K}, \mathbf{G}_{m}\right) \rightarrow \boldsymbol{\Gamma}\left(\mathcal{O}_{K}, \mathcal{G}_{m}\right)=\boldsymbol{\Gamma}\left(K, \mathbf{G}_{m}\right) \rightarrow \boldsymbol{\Gamma}\left(\mathcal{O}_{K}, \mathbb{Z}_{x}\right) \rightarrow 0
$$

coming from the exact sequence $0 \rightarrow \mathbf{G}_{m} \rightarrow \mathcal{G}_{m} \rightarrow \mathbb{Z}_{x} \rightarrow 0$ of group schemes over $\mathcal{O}_{K}$, where $\mathcal{G}_{m}$ is the Néron (lft) model of $\mathbf{G}_{m}$ and $\mathbb{Z}_{x}$ the étale group with support on $x=$ Spec $k$ and special fiber $\mathbb{Z}$, and we used (3.1.3) (C) for the middle isomorphism and (3.4.1) for the exactness. On $k$-points, it is the usual sequence $0 \rightarrow U_{K} \rightarrow K^{\times} \rightarrow \mathbb{Z} \rightarrow 0$, where $U_{K}=\mathcal{O}_{K}^{\times}$. We call the composite

$$
R \boldsymbol{\Gamma}\left(K, \mathbf{G}_{m}\right)=\mathbf{K}^{\times} \rightarrow \mathbb{Z}
$$

the trace morphism.

Let $A$ be an abelian variety over $K$ with dual $A^{\vee}$. Recall from (3.4.3) (d) that $\boldsymbol{\Gamma}(A) \in \mathrm{PAlg} / k, \mathbf{H}^{1}(A) \in \mathrm{IAlg}_{\mathrm{uc}} / k, \mathbf{H}^{n}(A)=0$ for $n \geq 2$, and $R \boldsymbol{\Gamma}(A)$ is P-acyclic. In particular, we write $R \tilde{\boldsymbol{\Gamma}}(A)=R \boldsymbol{\Gamma}(A)$. With the morphism of functoriality of 
$R \tilde{\boldsymbol{\Gamma}}$ in (3.3.8) and the trace morphism above, we have morphisms

$$
\begin{aligned}
R \boldsymbol{\Gamma}\left(A^{\vee}\right) & \rightarrow R \tilde{\boldsymbol{\Gamma}} R \mathbf{H o m}_{K}\left(A, \mathbf{G}_{m}\right)[1] \\
& \rightarrow R \mathbf{H o m}_{k_{\text {proet }}^{\text {indrat }}}\left(R \boldsymbol{\Gamma}(A), R \boldsymbol{\Gamma}\left(\mathbf{G}_{m}\right)\right)[1] \\
& \rightarrow R \mathbf{H o m}_{k_{\text {proet }}^{\text {indrat }}}(R \boldsymbol{\Gamma}(A), \mathbb{Z})[1]=R \boldsymbol{\Gamma}(A)^{\mathrm{SD}}[1]
\end{aligned}
$$

in $D\left(k_{\text {proet }}^{\text {indrat }}\right)$. We denote by $\vartheta_{A}$ the composite morphism:

$$
\vartheta_{A}: R \boldsymbol{\Gamma}\left(A^{\vee}\right) \rightarrow R \boldsymbol{\Gamma}(A)^{\mathrm{SD}}[1] \quad\left(=R \mathbf{H o m}_{k_{\mathrm{proet}}^{\text {indrat }}}(R \boldsymbol{\Gamma}(A), \mathbb{Q} / \mathbb{Z})\right) .
$$

Recall from (3.4.3) (d) and (3.4.2) (1a) that there are surjections

$$
\Gamma(A) \rightarrow \mathcal{A}_{x} \rightarrow \pi_{0}\left(\mathcal{A}_{x}\right)
$$

with connected kernels, where $\mathcal{A}$ is the Néron model of $A$ and $\mathcal{A}_{x}$ its special fiber. In particular, $\boldsymbol{\Gamma}(A)$ is not Serre reflexive in general due to the possibly non-zero semi-abelian part of $\mathcal{A}_{x}$. We take the Serre dual of $\vartheta_{A}$. Replacing $A$ with $A^{\vee}$, we have a morphism

$$
\theta_{A}: R \boldsymbol{\Gamma}\left(A^{\vee}\right)^{\mathrm{SDSD}} \rightarrow R \boldsymbol{\Gamma}(A)^{\mathrm{SD}}[1]
$$

in $D\left(k_{\text {proet }}^{\text {indrat }}\right)$. Then $\vartheta_{A}$ can be written as the composite of the natural evaluation morphism id $\rightarrow$ SDSD and $\theta_{A}$ :

$$
\vartheta_{A}: R \boldsymbol{\Gamma}\left(A^{\vee}\right) \rightarrow R \boldsymbol{\Gamma}\left(A^{\vee}\right)^{\mathrm{SDSD}} \stackrel{\theta_{A}}{\rightarrow} R \boldsymbol{\Gamma}(A)^{\mathrm{SD}}[1] .
$$

We have $\boldsymbol{\Gamma}(A)^{\mathrm{SD}} \in D^{b}\left(\operatorname{IAlg}_{\mathrm{uc}} / k\right)$ and $\mathbf{H}^{1}(A)^{\mathrm{SD}} \in D^{b}\left(\mathrm{PAlg}_{\mathrm{uc}} / k\right)$ by (2.4.1), which are both Serre reflexive. Therefore $R \boldsymbol{\Gamma}(A)^{\mathrm{SD}}$ is Serre reflexive, so is its Serre dual. Therefore $\theta_{A}$ is a morphism between Serre reflexive complexes. Now we can precisely state our duality theorem for abelian varieties.

Theorem (4.1.2). The morphism $\theta_{A}$ defined above is an isomorphism.

We start proving this from the next subsection. The proof finishes at $\$ 7$

4.2. Reduction to components groups and the first cohomology. First, the sheaf $\mathbf{H}^{1}(A) \in \mathrm{IAlg} / k$ is $\mathrm{P}$-acyclic as we saw. Since $\boldsymbol{\Gamma}(A) \in \mathrm{PAlg} / k$, the sheaf $\operatorname{Ext}_{k_{\mathrm{et}}^{\text {indrat }}}^{1}(\boldsymbol{\Gamma}(A), \mathbb{Q} / \mathbb{Z})$ is locally of finite presentation by what we saw after (2.4.1). In particular, $\mathbf{E x t}_{k_{\mathrm{et}}^{\text {indrat }}}^{1}(\boldsymbol{\Gamma}(A), \mathbb{Q} / \mathbb{Z})$ is $\mathrm{P}$-acyclic by (2.4.2) (国), and $\mathbf{E x t}_{k_{\mathrm{et}}^{\text {indrat }}}^{1}(\boldsymbol{\Gamma}(A), \mathbb{Q} / \mathbb{Z})$ and $\mathbf{E x t}_{k_{\mathrm{proet}}}^{1 \text { indrat }}(\boldsymbol{\Gamma}(A), \mathbb{Q} / \mathbb{Z})$ are equal as functors on $k^{\text {indrat }}$.

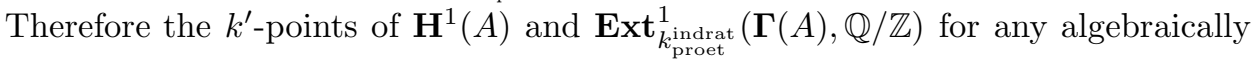
closed field $k^{\prime} \in k^{\text {indrat }}$ are $H^{1}\left(\mathbf{K}\left(k^{\prime}\right), A\right)$ and $\operatorname{Ext}_{k_{\text {proet }}^{\text {indrat }}}^{1}(\boldsymbol{\Gamma}(A), \mathbb{Q} / \mathbb{Z})$, respectively.

Proposition (4.2.1). The morphism $\theta_{A}$ induces two morphisms

$$
\begin{gathered}
\theta_{A}^{+0}: \pi_{0}\left(\mathcal{A}_{x}^{\vee}\right) \rightarrow \pi_{0}\left(\mathcal{A}_{x}\right)^{\mathrm{PD}} \quad \text { in } \mathrm{FEt} / k, \\
\theta_{A}^{+1}: \mathbf{H}^{1}\left(A^{\vee}\right) \rightarrow \mathbf{E x t}_{k_{\text {proet }}^{\text {indrat }}}^{1}(\boldsymbol{\Gamma}(A), \mathbb{Q} / \mathbb{Z}) \quad \text { in } \quad \mathrm{IAlg}_{\mathrm{uc}} / k .
\end{gathered}
$$

For any algebraically closed field $k^{\prime} \in k^{\text {indrat }}$, denote by $\theta_{A}^{+1}\left(k^{\prime}\right)$ the morphism $\theta_{A}^{+1}$ induced on the $k^{\prime}$-points:

$$
\theta_{A}^{+1}\left(k^{\prime}\right): H^{1}\left(\mathbf{K}\left(k^{\prime}\right), A^{\vee}\right) \rightarrow \operatorname{Ext}_{k_{\text {proet }}^{\prime \text { ind rat }}}^{1}(\boldsymbol{\Gamma}(A), \mathbb{Q} / \mathbb{Z}) .
$$

Then the following are equivalent:

- $\theta_{A}$ is an isomorphism.

- $\theta_{A}^{+0}, \theta_{A}^{+1}, \theta_{A^{\vee}}^{+1}$ are isomorphisms. 
- $\theta_{A}^{+0}, \theta_{A}^{+1}\left(k^{\prime}\right), \theta_{A^{\vee}}^{+1}\left(k^{\prime}\right)$ are isomorphisms for any algebraically closed field $k^{\prime} \in k^{\text {indrat }}$.

We prove this in this subsection. Basically the morphisms will be obtained by writing down the spectral sequence associated with $\theta_{A}$, as we did in 1.2 More precisely, $\theta_{A}^{+0}$ is the morphism induced on the $\pi_{0}$ of $H^{0}$ of the both sides of $\theta_{A}$ and $\theta_{A}^{+1}$ is the morphism induced on the $H^{1}$. The part for $H^{-1}$ and the identity component of $H^{0}$ contain no additional information by symmetry, and $H^{n}=0$ for the both sides for $n \neq-1,0,1$. To clarify the symmetry mentioned and the treatment of the double-dual, we split the construction and the proof into several steps.

Consider the morphisms

$$
\varliminf_{n}^{\varliminf_{n}} \boldsymbol{\Gamma}(A) \rightarrow \boldsymbol{\Gamma}(A) \rightarrow R \boldsymbol{\Gamma}(A),
$$

where the (non-derived) limit on the left is over multiplication by $n \geq 1$. Note that $\lim _{n} \boldsymbol{\Gamma}(A)$ is no longer P-acyclic. There is a canonical choice of a mapping cone of the composite $\lim _{n} \boldsymbol{\Gamma}(A) \rightarrow R \boldsymbol{\Gamma}(A)$ in $D\left(k_{\text {proet }}^{\text {indrat }}\right)$ since the former is concentrated in degree 0 and the latter is concentrated in non-negative degrees. We denote this mapping cone by $\left[\lim _{n} \boldsymbol{\Gamma}(A) \rightarrow R \boldsymbol{\Gamma}(A)\right]$.

Proposition (4.2.2). There is a canonical isomorphism

$$
\left[\lim _{n} \Gamma(A) \rightarrow R \boldsymbol{\Gamma}(A)\right]=R \boldsymbol{\Gamma}(A)^{\mathrm{SDSD}} \text {. }
$$

The induced morphism $R \boldsymbol{\Gamma}(A) \rightarrow R \boldsymbol{\Gamma}(A)^{\mathrm{SDSD}}$ is the natural evaluation morphism.

Proof. Since $\boldsymbol{\Gamma}(A) \in \mathrm{PAlg} / k$, we have $\left(\lim _{n} \boldsymbol{\Gamma}(A)\right)^{\mathrm{SD}}=0$ as seen in the proof of (2.4.1) (d). Therefore we have a natural morphism and an isomorphism

$$
\left[\varliminf_{n}^{\lim } \boldsymbol{\Gamma}(A) \rightarrow R \boldsymbol{\Gamma}(A)\right] \rightarrow\left[\stackrel{\lim }{n}_{\boldsymbol{L}} \boldsymbol{\Gamma}(A) \rightarrow R \boldsymbol{\Gamma}(A)\right]^{\mathrm{SDSD}}=R \boldsymbol{\Gamma}(A)^{\mathrm{SDSD}} .
$$

We need to show that the left term is Serre reflexive. Since $\mathbf{H}^{n}(A)=0$ for $n \geq 2$, we have a distinguished triangle

$$
\left[\varliminf_{n}^{\lim } \boldsymbol{\Gamma}(A) \rightarrow \boldsymbol{\Gamma}(A)\right] \rightarrow\left[\overleftarrow{n}_{n} \boldsymbol{\operatorname { l i m }} \boldsymbol{\Gamma}(A) \rightarrow R \boldsymbol{\Gamma}(A)\right] \rightarrow \mathbf{H}^{1}(A)[-1] .
$$

The left mapping cone is isomorphic to $\Gamma(A)^{\mathrm{SDSD}} \in D^{b}\left(\mathrm{PAlg}_{\mathrm{uc}} / k\right)$ by (2.4.1) (d), which is Serre reflexive. We have $\mathbf{H}^{1}(A) \in \mathrm{IAlg}_{\mathrm{uc}} / k$, which is Serre reflexive. Therefore the middle term is Serre reflexive. Thus we get the required isomorphism.

Let $\boldsymbol{\Gamma}\left(A^{\vee}\right)_{0}$ be the identity component of $\boldsymbol{\Gamma}\left(A^{\vee}\right)$. We have two distinguished triangles

$$
\begin{aligned}
& \boldsymbol{\Gamma}\left(A^{\vee}\right)_{0} \longrightarrow R \boldsymbol{\Gamma}\left(A^{\vee}\right) \longrightarrow\left[\boldsymbol{\Gamma}\left(A^{\vee}\right)_{0} \rightarrow R \boldsymbol{\Gamma}\left(A^{\vee}\right)\right] \\
& \mathbf{H}^{1}(A)^{\mathrm{SD}}[2] \longrightarrow R \boldsymbol{\Gamma}(A)^{\mathrm{SD}}[1] \longrightarrow \quad \boldsymbol{\Gamma}(A)^{\mathrm{SD}}[1]
\end{aligned}
$$

and the morphism $\vartheta_{A}: R \boldsymbol{\Gamma}\left(A^{\vee}\right) \rightarrow R \boldsymbol{\Gamma}(A)^{\mathrm{SD}}[1]$ between the middle terms. 
Proposition (4.2.3). There is a unique way to extend $\vartheta_{A}$ to a morphism of triangles

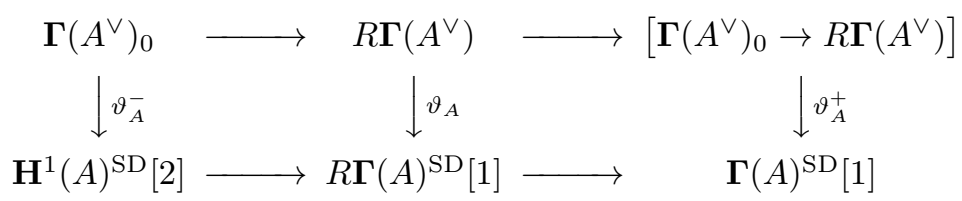

(which in particular means that $\vartheta_{A}^{+}$and $\vartheta_{A}^{-}$are compatible with the connecting morphisms of the triangles). This diagram can further be extended uniquely to a morphism of triangles

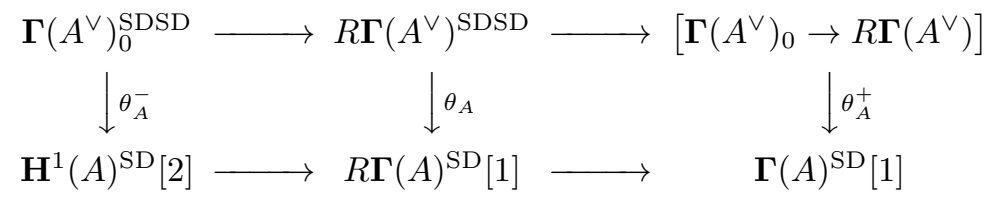

(where the middle $\theta_{A}$ has been defined earlier.)

Proof. Since $\boldsymbol{\Gamma}(A) \in \mathrm{PAlg} / k$, the complex $\boldsymbol{\Gamma}(A)^{\mathrm{SD}}[1]=R \operatorname{Hom}_{k_{\mathrm{protat}}^{\text {indrat }}}(\boldsymbol{\Gamma}(A), \mathbb{Q} / \mathbb{Z})$ is concentrated in degrees 0 and 1 with $H^{0}=\pi_{0}(\boldsymbol{\Gamma}(A))^{\mathrm{PD}}=\pi_{0}\left(\mathcal{A}_{x}\right)^{\mathrm{PD}} \in \mathrm{FEt} / k$ by (2.4.1) (国), (b) and (d) . In particular, its $(-1)$-shift $\boldsymbol{\Gamma}(A)^{\mathrm{SD}}$ is concentrated in degrees 1 and 2. Therefore

$$
\operatorname{Hom}_{D\left(k_{\text {proet }}^{\text {indrat }}\right)}\left(\boldsymbol{\Gamma}\left(A^{\vee}\right)_{0}, \boldsymbol{\Gamma}(A)^{\mathrm{SD}}[1]\right)=\operatorname{Hom}_{D\left(k_{\text {proet }}^{\text {indrat }}\right)}\left(\boldsymbol{\Gamma}\left(A^{\vee}\right)_{0}, \boldsymbol{\Gamma}(A)^{\mathrm{SD}}\right)=0,
$$

since a morphism in $D\left(k_{\text {proet }}^{\text {indrat }}\right)$ from an object of $\mathrm{Ab}\left(k_{\text {proet }}^{\text {indrat }}\right)$ to a complex concentrated in non-negative degrees factors through $H^{0}$. With this, we can uniquely extend $\vartheta_{A}$ to the first diagram by the general lemma below. For the second diagram, it is sufficient to note that the right upper term (whose $H^{0}=\pi_{0}\left(\boldsymbol{\Gamma}\left(A^{\vee}\right)\right.$ ) is finite), the left bottom term, the middle bottom term and the right lower term are all Serre reflexive by (2.4.1) (b), (d).

Lemma (4.2.5). Let $\mathcal{D}$ be a triangulated category. Let $X \rightarrow Y \rightarrow Z$ and $X^{\prime} \rightarrow$ $Y^{\prime} \rightarrow Z^{\prime}$ be two distinguished triangles in $\mathcal{D}$. Assume that the homomorphisms $\operatorname{Hom}\left(X, X^{\prime}\right) \rightarrow \operatorname{Hom}\left(X, Y^{\prime}\right)$ and $\operatorname{Hom}\left(Z, Z^{\prime}\right) \rightarrow \operatorname{Hom}\left(Y, Z^{\prime}\right)$ are isomorphisms. Then any morphism $f: Y \rightarrow Y^{\prime}$ can uniquely be extended to a morphism of the triangles. The assumption is satisfied if $\operatorname{Hom}\left(X, Z^{\prime}\right)=\operatorname{Hom}\left(X, Z^{\prime}[-1]\right)=0$.

Proof. This is elementary and well-known. (See also KS06, Prop. 10.1.17].) We recall its proof. Let $g: X \rightarrow X^{\prime}$ be the morphism corresponding to the composite of $X \rightarrow Y$ and $f: Y \rightarrow Y^{\prime}$ under the isomorphism $\operatorname{Hom}\left(X, X^{\prime}\right) \stackrel{\sim}{\rightarrow} \operatorname{Hom}\left(X, Y^{\prime}\right)$. Then by an axiom of triangulated categories, there is a morphism $h: Z \rightarrow Z^{\prime}$ such that the triple $(g, f, h)$ is a morphism of the triangles. This in particular means that the image of $h$ under the isomorphism $\operatorname{Hom}\left(Z, Z^{\prime}\right) \stackrel{\sim}{\rightarrow} \operatorname{Hom}\left(Y, Z^{\prime}\right)$ is the composite of $f: Y \rightarrow Y^{\prime}$ and $Y^{\prime} \rightarrow Z^{\prime}$. Hence such a morphism $h$ is unique. This shows that $f$ is uniquely extended to a morphism of the triangles. If $\operatorname{Hom}\left(X, Z^{\prime}\right)=$ $\operatorname{Hom}\left(X, Z^{\prime}[-1]\right)=0$, then the assumption follows from the long exact sequence of Hom. 
Proposition (4.2.6). The morphism $\theta_{A}^{+}$in (4.2.4) induces morphisms

$$
\begin{gathered}
\theta_{A}^{+0}: \pi_{0}\left(\mathcal{A}_{x}^{\vee}\right) \rightarrow \pi_{0}\left(\mathcal{A}_{x}\right)^{\mathrm{PD}} \quad \text { in } \mathrm{FEt} / k, \\
\theta_{A}^{+1}: \mathbf{H}^{1}\left(A^{\vee}\right) \rightarrow \mathbf{E x t}_{k_{\text {proet }}^{\text {indrat }}}^{1}(\boldsymbol{\Gamma}(A), \mathbb{Q} / \mathbb{Z}) \quad \text { in } \quad \mathrm{IAlg}_{\mathrm{uc}} / k,
\end{gathered}
$$

on $H^{0}, H^{1}$, respectively.

Proof. This follows from $\boldsymbol{\Gamma}(A)^{\mathrm{SD}}[1]=R \mathbf{H o m}_{k_{\text {proet }}^{\text {indrat }}}(\boldsymbol{\Gamma}(A), \mathbb{Q} / \mathbb{Z}), \pi_{0}(\boldsymbol{\Gamma}(A))=\pi_{0}\left(\mathcal{A}_{x}\right)$, and (2.4.1) (b).

Proposition (4.2.7). The morphism $\theta_{A}$ is an isomorphism if and only if $\theta_{A}^{+}$and $\theta_{A}^{-}$are so.

Proof. The "if" part is trivial. We show the converse. We have $\lim _{n} \boldsymbol{\Gamma}\left(A^{\vee}\right)=$ $\lim _{n} \boldsymbol{\Gamma}\left(A^{\vee}\right)_{0}$ since finite groups are killed by $\lim _{n}$. By (2.4.1) (d) and (4.2.2), we have

$$
\begin{gathered}
\boldsymbol{\Gamma}\left(A^{\vee}\right)_{0}^{\mathrm{SDSD}}=\left[\varliminf_{n} \boldsymbol{\Gamma}\left(A^{\vee}\right) \rightarrow \boldsymbol{\Gamma}\left(A^{\vee}\right)_{0}\right], \\
R \boldsymbol{\Gamma}(A)^{\mathrm{SDSD}}=\left[{\underset{\leftarrow}{n}}_{\lim } \boldsymbol{\Gamma}(A) \rightarrow R \boldsymbol{\Gamma}(A)\right] .
\end{gathered}
$$

From these, we can see that the upper triangle in the diagram (4.2.4) is of the form $X \rightarrow Y \rightarrow Z$ with: $X$ concentrated in degrees $-1,0 ; Y$ in $-1,0,1$; and $Z$ in 0,1 . For such a distinguished triangle, we have isomorphisms and an exact sequence

$$
\begin{gathered}
H^{-1}(X)=H^{-1}(Y), \quad H^{1}(Y)=H^{1}(Z), \\
0 \rightarrow H^{0}(X) \rightarrow H^{0}(Y) \rightarrow H^{0}(Z) \rightarrow 0 .
\end{gathered}
$$

The exact sequence in the second line is a connected-étale sequence in $\mathrm{PAlg} \mathrm{uc}_{\mathrm{uc}}$.

We show a similar statement for the lower triangle in the diagram (4.2.4): it has cohomologies in the same ranges with the same property (i.e. $H^{0}$ is a connected-étale sequence). Since $\mathbf{H}^{1}(A) \in \operatorname{IAlg}_{\mathrm{uc}} / k$, we know that $\mathbf{H}^{1}(A)^{\mathrm{SD}}[2]$ is concentrated in degrees $-1,0$ whose $H^{0}$ is connected pro-unipotent by (2.4.1) (b). We already saw that $\boldsymbol{\Gamma}(A)^{\mathrm{SD}}[1]$ is concentrated in degrees 0,1 whose $H^{0}$ is finite étale. Therefore $R \boldsymbol{\Gamma}(A)^{\mathrm{SD}}[1]$ is concentrated in degrees $-1,0,1$ whose $H^{0}$ is in $\mathrm{PAlg}$ uc. Thus the lower triangle in (4.2.4) has the expected properties.

Now if the morphism on the $Y$ 's of the two triangles in (4.2.4) is an isomorphism, then so are the morphisms on the $X$ 's and $Z$ 's.

Before the next proposition, note that, by (2.4.1) (国),

$$
\begin{aligned}
\boldsymbol{\Gamma}(A)_{0}^{\mathrm{SD}} & =R \mathbf{H o m}_{k_{\text {proet }}^{\text {indrat }}}\left(\boldsymbol{\Gamma}(A)_{0}, \mathbb{Q} / \mathbb{Z}\right)[-1] \\
& =\mathbf{E x t}_{k_{\text {proet }}^{\text {indrat }}\left(\boldsymbol{\Gamma}(A)_{0}, \mathbb{Q} / \mathbb{Z}\right)[-2] .} \\
& =\mathbf{E x t}_{k_{\text {proet }}^{\text {indrat }}}(\boldsymbol{\Gamma}(A), \mathbb{Q} / \mathbb{Z})[-2] .
\end{aligned}
$$

Hence the Serre dual (up to shift) of $\theta_{A}^{+1}$ in (4.2.6) can be written as a morphism $\boldsymbol{\Gamma}(A)_{0}^{\mathrm{SDSD}} \rightarrow \mathbf{H}^{1}\left(A^{\vee}\right)^{\mathrm{SD}}[2]$. Since $\theta_{A^{\vee}}$ is also a morphism $\boldsymbol{\Gamma}(A)_{0}^{\mathrm{SDSD}} \rightarrow$ $\mathbf{H}^{1}\left(A^{\vee}\right)^{\mathrm{SD}}[2]$, it is meaningful to compare $\theta_{A^{\vee}}^{-}$and $\left(\theta_{A}^{+1}\right)^{\mathrm{SD}}$.

Proposition (4.2.8). We have $\theta_{A^{\vee}}=\left(\theta_{A}\right)^{\mathrm{SD}}, \theta_{A^{\vee}}^{-}=\left(\theta_{A}^{+1}\right)^{\mathrm{SD}}$ and $\theta_{A^{\vee}}^{+0}=\left(\theta_{A}^{+0}\right)^{\mathrm{PD}}$. 
Proof. We first show $\theta_{A^{\vee}}=\left(\theta_{A}\right)^{\mathrm{SD}}$, or $\theta_{A}=\left(\theta_{A^{\vee}}\right)^{\mathrm{SD}}$. The morphisms $A^{\vee} \otimes^{L} A \rightarrow$ $\mathbf{G}_{m}[1]$ and $A^{\vee} \otimes^{L} A^{\vee \vee} \rightarrow \mathbf{G}_{m}[1]$ in $D\left(K_{\mathrm{fppf}}\right)$ coming from the Poincaré bundle (or $\mathbf{G}_{m}$-biextension) are compatible with the biduality isomorphism $A \stackrel{\sim}{\rightarrow} A^{\vee \vee}$. Hence we have a commutative diagram

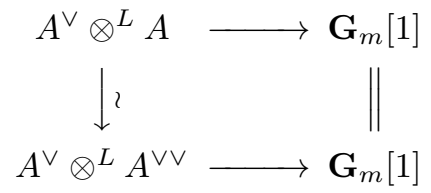

in $D\left(K_{\mathrm{fppf}} / k_{\mathrm{et}}^{\text {indrat }}\right)$. Recall from the second paragraph after (3.3.3) that the morphism of functoriality and the cup product pairing are equivalent under the derived tensor-hom adjunction [KS06. Thm. 18.6.4 (vii)]. This relation also holds after proétale sheafification. Applying $R \tilde{\boldsymbol{\Gamma}}$ to the above diagram and using the cup product pairing, we have a commutative diagram

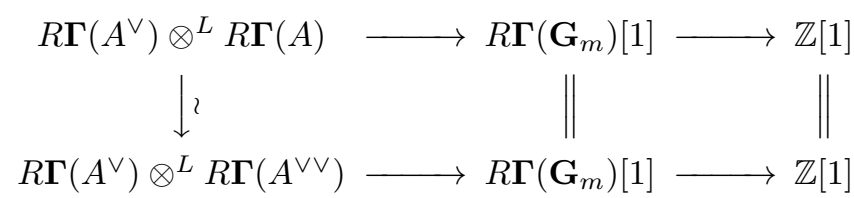

in $D\left(k_{\text {proet }}^{\text {indrat }}\right)$. The upper morphisms give a morphism $R \boldsymbol{\Gamma}\left(A^{\vee}\right)^{\mathrm{SDSD}} \rightarrow R \boldsymbol{\Gamma}(A)^{\mathrm{SD}}$ via the derived tensor-Hom adjunction, which is $\theta_{A}$. Similarly, the lower morphisms give a morphism $R \boldsymbol{\Gamma}\left(A^{\vee \vee}\right) \rightarrow R \boldsymbol{\Gamma}\left(A^{\vee}\right)^{\mathrm{SD}}$, which is $\vartheta_{A^{\vee}}$, and a morphism $R \boldsymbol{\Gamma}\left(A^{\vee}\right)^{\mathrm{SDSD}} \rightarrow R \boldsymbol{\Gamma}\left(A^{\vee \vee}\right)^{\mathrm{SD}}$, which is $\left(\theta_{A^{\vee}}\right)^{\mathrm{SD}}$. The commutativity of the diagram implies that $\theta_{A}=\left(\theta_{A^{\vee}}\right)^{\mathrm{SD}}$.

For $\theta_{A^{\vee}}^{-}=\left(\theta_{A}^{+1}\right)^{\mathrm{SD}}$, or $\left(\theta_{A}^{-}\right)^{\mathrm{SD}}=\theta_{A^{\vee}}^{+1}$, apply SD to the second diagram of (4.2.3):

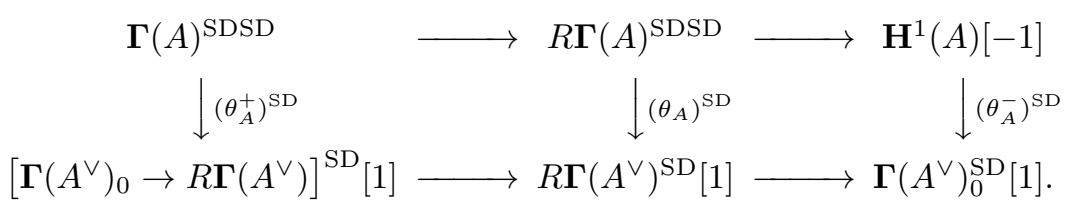

The morphism $\left(\theta_{A}^{-}\right)^{\mathrm{SD}}$ is a morphism between complexes concentrated in degree 1 . The complex $\boldsymbol{\Gamma}(A)^{\mathrm{SDSD}}$ is concentrated in degrees $-1,0$ by (2.4.1) (d). Consider the distinguished triangle

$$
\mathbf{H}^{1}\left(A^{\vee}\right)^{\mathrm{SD}}[2] \rightarrow\left[\boldsymbol{\Gamma}\left(A^{\vee}\right)_{0} \rightarrow R \boldsymbol{\Gamma}\left(A^{\vee}\right)\right]^{\mathrm{SD}}[1] \rightarrow \pi_{0}\left(\boldsymbol{\Gamma}\left(A^{\vee}\right)\right)^{\mathrm{PD}} .
$$

By $\mathbf{H}^{1}\left(A^{\vee}\right) \in \mathrm{IAlg}_{\mathrm{uc}} / k$ and (2.4.1) (b), we know that $\mathbf{H}^{1}\left(A^{\vee}\right)^{\mathrm{SD}}[2]$ is concentrated in degrees $-1,0$. Hence the middle term $\left[\boldsymbol{\Gamma}\left(A^{\vee}\right)_{0} \rightarrow R \boldsymbol{\Gamma}\left(A^{\vee}\right)\right]^{\mathrm{SD}}[1]$ is concentrated in degrees $-1,0$. Therefore $H^{1}\left(\left(\theta_{A}\right)^{\mathrm{SD}}\right)=\left(\theta_{A}^{-}\right)^{\mathrm{SD}}$. By a similar observation, we have $H^{1}\left(\theta_{A^{\vee}}\right)=\theta_{A^{\vee}}^{+1}$. Hence $\left(\theta_{A}\right)^{\mathrm{SD}}=\theta_{A^{\vee}}$ proved above implies $\left(\theta_{A}^{-}\right)^{\mathrm{SD}}=\theta_{A^{\vee}}^{+1}$.

Finally, the equality $\theta_{A^{\vee}}=\left(\theta_{A}\right)^{\mathrm{SD}}$ induces the equality $\theta_{A^{\vee}}^{+0}=\left(\theta_{A}^{+0}\right)^{\mathrm{PD}}$ on $\pi_{0} H^{0}$.

The previous two propositions show that the first two assertions in (4.2.1) are equivalent. To connect them to the third, it is enough to show the following general lemma.

Lemma (4.2.9). Let $\varphi: B \rightarrow C$ be a morphism in IAlg $/ k$. Assume that $\varphi: B\left(k^{\prime}\right) \rightarrow$ $C\left(k^{\prime}\right)$ is an isomorphism for any algebraically closed field $k^{\prime}$ over $k$. Then $\varphi: B \rightarrow C$ is an isomorhism. 
Proof. By considering the kernel and cokernel, it is enough to show that if $D \in$ IAlg $/ k$ satisfies $D\left(k^{\prime}\right)=0$ for any algebraically closed field $k^{\prime}$ over $k$, then $D=0$. For this, it is enough to show that $\operatorname{Hom}(E, D)=0$ for any $E \in \operatorname{Alg} / k$. Let $\xi_{E}$ be the generic point of $E$. Then by (3.4.5), we have

$$
\operatorname{Hom}(E, D)=\operatorname{Ker}\left(D\left(\xi_{E}\right) \stackrel{\sigma}{\rightarrow} D\left(\xi_{E \times{ }_{k} E}\right)\right)
$$

(with the same notation as the cited proof). But the assumption implies that $D\left(\xi_{E}\right)=0$. Hence $\operatorname{Hom}(E, D)=0$ and $D=0$.

Remark (4.2.10). Here is another method to obtain (4.2.1) or, more precisely, (4.2.3). The Poincaré biextension $A^{\vee} \times A \rightarrow \mathbf{G}_{m}[1]$ canonically extends to a biextension $\mathcal{A}_{0}^{\vee} \times \mathcal{A} \rightarrow \mathbf{G}_{m}$ [1] by Gro72, IX, 1.4.3], where $\mathcal{A}_{0}^{\vee}$ is the maximal open subgroup scheme of $\mathcal{A}^{\vee}$ with connected special fiber. Hence we have a morphism $\mathcal{A}_{0}^{\vee} \rightarrow R \mathbf{H o m}_{\mathcal{O}_{K}}\left(\mathcal{A}, \mathbf{G}_{m}[1]\right)$. We have the trace isomorphism $R \boldsymbol{\Gamma}_{x}\left(\mathcal{O}_{K}, \mathbf{G}_{m}[1]\right)=$ $\mathbb{Z}$; see the second paragraph of $\$ 5.2 .1$. Hence we have a morphism

$$
\begin{aligned}
R \boldsymbol{\Gamma}_{x}\left(\mathcal{O}_{K}, \mathcal{A}_{0}^{\vee}\right) & \rightarrow R \mathbf{H o m}_{k_{\text {proet }}^{\text {indrat }}}\left(R \boldsymbol{\Gamma}\left(\mathcal{O}_{K}, \mathcal{A}\right), R \boldsymbol{\Gamma}_{x}\left(\mathcal{O}_{K}, \mathbf{G}_{m}[1]\right)\right) \\
& =R \mathbf{H o m}_{k_{\text {proet }}^{\text {indrat }}}\left(R \boldsymbol{\Gamma}\left(\mathcal{O}_{K}, \mathcal{A}\right), \mathbb{Z}\right)=R \boldsymbol{\Gamma}\left(\mathcal{O}_{K}, \mathcal{A}\right)^{\mathrm{SD}} .
\end{aligned}
$$

The localization exact sequences give a morphism

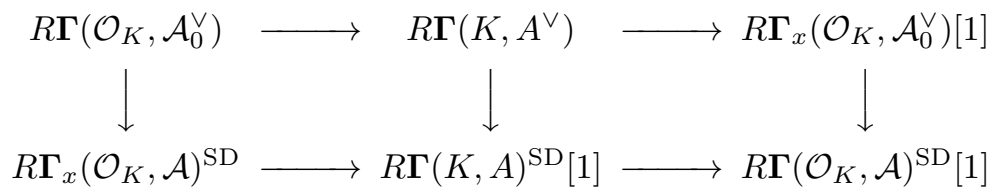

between distinguished triangles (see (5.2.2.2) for the corresponding statement for finite flat group schemes). We can identify this diagram with the first diagram in (4.2.3). We somewhat avoided the use of cohomology of $\mathcal{O}_{K}$ in this subsection and constructed the diagram purely from the structure of $R \boldsymbol{\Gamma}(K, A)$ as a complex of sheaves over $k$.

\section{Relation to Grothendieck's and Šafarevič's CONJECtures}

We compare our morphisms $\theta_{A}^{+0}, \theta_{A}^{+1}$ with Grothendieck's pairing, Bégueri's isomorphism and Bester-Bertapelle's isomorphism.

\subsection{Grothendieck's pairing.}

Proposition (5.1.1). The morphism

$$
\theta_{A}^{+0}: \pi_{0}\left(\mathcal{A}_{x}^{\vee}\right) \rightarrow \pi_{0}\left(\mathcal{A}_{x}\right)^{\mathrm{PD}},
$$

of (4.2.1) coincides with Grothendieck's pairing Gro72, IX, 1.2.1].

We prove this by writing down $\theta_{A}^{+0}$ explicitly. Recall that the morphism $\theta_{A}$ is defined by the functoriality of $R \tilde{\boldsymbol{\Gamma}}$ applied to $R \operatorname{Hom}_{K}\left(A, \mathbf{G}_{m}\right)$ and the trace morphism $R \boldsymbol{\Gamma}\left(\mathbf{G}_{m}\right) \rightarrow \mathbb{Z}$ or the valuation map $\mathbf{K}^{\times} \rightarrow \mathbb{Z}$. To write $\theta_{A}^{+0}$, we give an arbitrary element of $\operatorname{Ext}_{K}^{1}\left(A, \mathbf{G}_{m}\right)$, which is an extension class, and apply $R \tilde{\boldsymbol{\Gamma}}$.

Proof. We may assume that $k$ is algebraically closed. We first recall the construction of Grothendieck's pairing following [Mil06, Appendix C]. We denote by $j:$ Spec $K_{\mathrm{sm}} \hookrightarrow \operatorname{Spec} \mathcal{O}_{K \text {,sm }}$ the natural morphism between the smooth sites. Let $0 \rightarrow \mathbf{G}_{m} \rightarrow X \rightarrow A \rightarrow 0$ be an element of $\Gamma\left(K, A^{\vee}\right)=\operatorname{Ext}_{K}^{1}\left(A, \mathbf{G}_{m}\right)$. Let $\mathcal{G}_{m}=j_{*} \mathbf{G}_{m}, \mathcal{X}=j_{*} X, \mathcal{A}=j_{*} A$ be the Néron models (or more precisely, the 
Néron lft models). Then the sequence $0 \rightarrow \mathcal{G}_{m} \rightarrow \mathcal{X} \rightarrow \mathcal{A} \rightarrow 0$ in $\operatorname{Ab}\left(\mathcal{O}_{K, \mathrm{sm}}\right)$ is exact since $R^{1} j_{*} \mathbf{G}_{m}=0$ (see the proof of [Mil06, loc.cit., Lem.C.10]). We can view this as an exact sequence of group schemes over $\mathcal{O}_{K}$. Hence we have an exact sequence $0 \rightarrow \mathcal{G}_{m} \rightarrow \mathcal{X} \rightarrow \mathcal{A} \rightarrow 0$ in $\operatorname{Ab}\left(\mathcal{O}_{K, \text { fppf }}\right)$ (though these no longer represent the pushforward sheaves of the original algebraic groups over $\left.K_{\mathrm{fppf}}\right)$. We also have an exact sequence $0 \rightarrow \mathbf{G}_{m} \rightarrow \mathcal{G}_{m} \rightarrow i_{*} \mathbb{Z} \rightarrow 0$ in $\mathrm{Ab}\left(\mathcal{O}_{K \text {,fppf }}\right)$, where $i$ : Spec $k_{\mathrm{fppf}} \hookrightarrow \operatorname{Spec} \mathcal{O}_{K, \text { fppf }}$ is the natural morphism. By pushing out the extension $0 \rightarrow \mathcal{G}_{m} \rightarrow \mathcal{X} \rightarrow \mathcal{A} \rightarrow 0$ by the morphism $\mathcal{G}_{m} \rightarrow i_{*} \mathbb{Z}$, we get an extension $0 \rightarrow i_{*} \mathbb{Z} \rightarrow \mathcal{X} / \mathbf{G}_{m} \rightarrow \mathcal{A} \rightarrow 0$ in $\operatorname{Ab}\left(\mathcal{O}_{K \text {,fppf }}\right)$. Pulling back by $i$, we have an exact sequence $0 \rightarrow \mathbb{Z} \rightarrow \mathcal{X}_{x} / \mathbf{G}_{m} \rightarrow \mathcal{A}_{x} \rightarrow 0$ in $\mathrm{Ab}\left(k_{\mathrm{fppf}}\right)$, where the subscript $x$ denotes the special fiber. Therefore we get an element of

$$
\operatorname{Ext}_{k_{\mathrm{fppf}}}^{1}\left(\mathcal{A}_{x}, \mathbb{Z}\right)=\operatorname{Hom}_{k_{\mathrm{fpp}}}\left(\mathcal{A}_{x}, \mathbb{Q} / \mathbb{Z}\right)=\pi_{0}\left(\mathcal{A}_{x}\right)^{\mathrm{PD}} .
$$

This defines a homomorphism $\Gamma\left(K, A^{\vee}\right)=\Gamma\left(\mathcal{O}_{K}, \mathcal{A}^{\vee}\right) \rightarrow \pi_{0}\left(\mathcal{A}_{x}\right)^{\mathrm{PD}}$, which turns out to factor through $\pi_{0}\left(\mathcal{A}_{x}^{\vee}\right)$ ([Mil06, loc.cit., Lem. C.11]). The homomorphism $\pi_{0}\left(\mathcal{A}_{x}^{\vee}\right) \rightarrow \pi_{0}\left(\mathcal{A}_{x}\right)^{\mathrm{PD}}$ thus obtained is Grothendieck's pairing.

We next describe $\theta_{A}^{+0}$. By definition, the morphism $\vartheta_{A}$ after taking $R \Gamma(k, \cdot)$ and $H^{0}$ is

$$
\begin{aligned}
\Gamma\left(K, A^{\vee}\right) & \rightarrow \operatorname{Hom}_{D\left(K_{\mathrm{fpp}} / k_{\mathrm{et}}^{\text {indrat }}\right)}\left(A, \mathbf{G}_{m}[1]\right) \\
& \stackrel{R \tilde{\boldsymbol{\Gamma}}}{\longrightarrow} \operatorname{Hom}_{D\left(k_{\mathrm{proet}}^{\text {indrat }}\right)}\left(R \boldsymbol{\Gamma}(A), R \boldsymbol{\Gamma}\left(\mathbf{G}_{m}\right)[1]\right) \\
& \stackrel{\text { trace }}{\longrightarrow} \operatorname{Hom}_{D\left(k_{\text {proet }}^{\text {indrat }}\right)}(\boldsymbol{\Gamma}(A), \mathbb{Z}[1]) .
\end{aligned}
$$

Since $R \operatorname{Hom}_{k_{\text {proet }}^{\text {indrat }}}(\boldsymbol{\Gamma}(A), \mathbb{Q})=0$ by $(2.3 .3)$ (d) , the final group in the above displayed equation is further isomorphic to

$$
\operatorname{Hom}_{k_{\text {proet }}^{\text {indrat }}}(\boldsymbol{\Gamma}(A), \mathbb{Q} / \mathbb{Z})=\operatorname{Hom}_{k_{\text {proet }}^{\text {indrat }}}\left(\pi_{0}\left(\mathcal{A}_{x}\right), \mathbb{Q} / \mathbb{Z}\right) .
$$

The composite $\Gamma\left(K, A^{\vee}\right) \rightarrow \pi_{0}\left(\mathcal{A}_{x}\right)^{\mathrm{PD}}$ factors through $\pi_{0}\left(\mathcal{A}_{x}^{\vee}\right)$ and the resulting homomorphism $\pi_{0}\left(\mathcal{A}_{x}^{\vee}\right) \rightarrow \pi_{0}\left(\mathcal{A}_{x}\right)^{\mathrm{PD}}$ is the definition of $\theta_{A}^{+0}$. Therefore an explicit description of $\theta_{A}^{+0}$ is given as follows. Let $0 \rightarrow \mathbf{G}_{m} \rightarrow X \rightarrow A \rightarrow 0$ be as above (which corresponds to a morphism $A \rightarrow \mathbf{G}_{m}[1]$ in $\left.D\left(K_{\mathrm{fppf}} / k_{\mathrm{et}}^{\text {indrat }}\right)\right)$. We apply $R \tilde{\boldsymbol{\Gamma}}$. We have $\mathbf{H}^{1}\left(\mathbf{G}_{m}\right)=0$ by (3.4.3) (国). Hence we have an exact sequence

$$
0 \rightarrow \mathbf{K}^{\times} \rightarrow \boldsymbol{\Gamma}(X) \rightarrow \boldsymbol{\Gamma}(A) \rightarrow 0
$$

in $\operatorname{Ab}\left(k_{\text {proet }}^{\text {indrat }}\right)$, which gives an element of $\operatorname{Ext}_{k_{\text {proet }}^{\text {indrat }}}^{1}\left(\boldsymbol{\Gamma}(A), \mathbf{K}^{\times}\right)$. By pushing it out by the valuation map $\mathbf{K}^{\times} \rightarrow \mathbb{Z}$, we have an exact sequence $0 \rightarrow \mathbb{Z} \rightarrow \boldsymbol{\Gamma}(X) / \mathbf{U}_{K} \rightarrow$ $\boldsymbol{\Gamma}(A) \rightarrow 0$. This gives a morphism $\boldsymbol{\Gamma}(A) \rightarrow \mathbb{Z}[1]$ in $D\left(k_{\text {proet }}^{\text {indrat }}\right)$ and hence a morphism $\pi_{0}\left(\mathcal{A}_{x}\right) \rightarrow \mathbb{Q} / \mathbb{Z}$, which is the value of $\theta_{A}^{+0}$.

Now we compare the two constructions. The sequence $0 \rightarrow \mathbf{K}^{\times} \rightarrow \boldsymbol{\Gamma}(X) \rightarrow$ $\boldsymbol{\Gamma}(A) \rightarrow 0$ above can also be obtained by applying $\boldsymbol{\Gamma}\left(\mathcal{O}_{K}, \cdot\right)$ to the sequence $0 \rightarrow \mathcal{G}_{m} \rightarrow \mathcal{X} \rightarrow \mathcal{A} \rightarrow 0$ of Néron models by (3.1.3) (ㄷ). We have a commutative diagram with exact rows

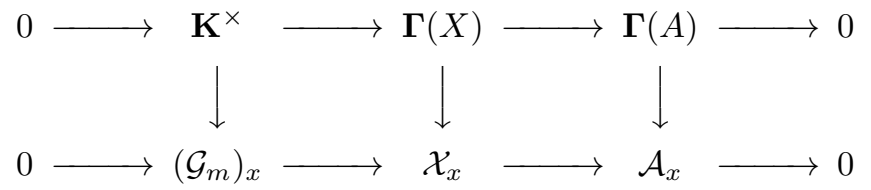


in $\operatorname{Ab}\left(k_{\text {proet }}^{\text {indrat }}\right)$, where the vertical morphisms are the reduction maps of the Néron models. The valuation map $\mathbf{K}^{\times} \rightarrow \mathbb{Z}$ and the morphism $\left(\mathcal{G}_{m}\right)_{x} \rightarrow \mathbb{Z}$ are compatible. Hence the pushouts by them yield the same morphism $\pi_{0}(\boldsymbol{\Gamma}(A))=\pi_{0}\left(\mathcal{A}_{x}\right) \rightarrow \mathbb{Q} / \mathbb{Z}$. Thus our $\theta_{A}^{+0}$ coincides with Grothendieck's pairing.

Remark (5.1.2). In the paragraph before the cited lemma Mil06, loc.cit., Lem. C.11], the cases $r=0,1$ of the equality

$$
\mathbf{E x t}_{\mathcal{O}_{K}}^{r}\left(\mathcal{A}, i_{*} \mathbb{Z}\right)=i_{*} \mathbf{E x t}_{k}^{r}\left(i^{*} \mathcal{A}, \mathbb{Z}\right)
$$

are stated and used. In this remark, we show, as an illustration of our methods in Suz13, that this equality is true for any $r$. The tricky point is that the topologies on $\mathcal{O}_{K}$ and $k$ here should be the smooth topologies and the $i$ here should be the natural continuous map $i_{\mathrm{sm}}$ : Spec $k_{\mathrm{sm}} \rightarrow \operatorname{Spec} \mathcal{O}_{K, \mathrm{sm}}$. Hence, if $r \geq 2$, the equality above might look as if it required that the pullback $i_{\mathrm{sm}}^{*}$ be exact or, equivalently, the pushforward $i_{\mathrm{sm}, *}$ send injectives to injectives. However, $i_{\mathrm{sm}}^{*}$ is not exact, since the category of smooth schemes over $\mathcal{O}_{K}$ is not closed under finite inverse limits. In fact, the morphism $\mathbf{G}_{a} \rightarrow \mathbf{G}_{a}$ over $\mathcal{O}_{K}$ given by multiplication by a prime element is injective in $\mathrm{Ab}\left(\mathcal{O}_{K, \mathrm{sm}}\right)$, while it becomes the zero map $\mathbf{G}_{a} \rightarrow \mathbf{G}_{a}$ after applying $i_{\mathrm{sm}}^{*}$. In the above proof of (5.1.1), we used the fact that $j_{*} X$ is representable (by the Néron model $\mathcal{X}$ ) to pass from the smooth site to the fppf site. Without the exactness of $i_{\mathrm{sm}}^{*}$, we can still prove the above equality for general $r$ as follows.

The last paragraph before (2.1.2) tells us that $i_{\mathrm{sm}, *}$ sends acyclic sheaves to acyclic sheaves. By Suz13, Lem. 3.7.2], we know that $i_{\mathrm{sm}}^{*}: \operatorname{Ab}\left(\mathcal{O}_{K, \mathrm{sm}}\right) \rightarrow \operatorname{Ab}\left(k_{\mathrm{sm}}\right)$ admits a left derived functor $L i_{\mathrm{sm}}^{*}: D\left(\mathcal{O}_{K, \mathrm{sm}}\right) \rightarrow D\left(k_{\mathrm{sm}}\right)$, which is left adjoint to $R i_{*}=i_{*}$. It is enough to show that $L_{n} i^{*} \mathcal{A}=0$ for any $n \geq 1$. Let $M(\mathcal{A})$ be Mac Lane's resolution of $\mathcal{A}$ in $\operatorname{Ab}\left(\mathcal{O}_{K, \mathrm{sm}}\right)$ (ML57, [Suz13, §3.4]). Its $n$-th term is a direct summand of a direct sum of sheaves of the form $\mathbb{Z}\left[\mathcal{A}^{m}\right]$ for various $m \geq 0$, where $\mathbb{Z}\left[\mathcal{A}^{m}\right]$ is the sheafification of the presheaf that sends a smooth $\mathcal{O}_{K}$-algebra $S$ to the free abelian group generated by the set $\mathcal{A}^{m}(S)$ (Suz13, $\S 3.4$, 3.5]). Hence we have $L i_{\mathrm{sm}}^{*} \mathcal{A}=i_{\mathrm{sm}}^{*} M(\mathcal{A})$, where $i_{\mathrm{sm}}^{*}$ on the right is applied termwise. We have $i_{\mathrm{sm}}^{*} \mathbb{Z}\left[\mathcal{A}^{m}\right]=\mathbb{Z}\left[i_{\mathrm{sm}}^{* \operatorname{set}}\left(\mathcal{A}^{m}\right)\right]$, where $i_{\mathrm{sm}}^{* \mathrm{set}}: \operatorname{Set}\left(\mathcal{O}_{K, \mathrm{sm}}\right) \rightarrow \operatorname{Set}\left(k_{\mathrm{sm}}\right)$ is the pullback for sheaves of sets. Since $\mathcal{A}^{m}$ is in the underlying category of Spec $\mathcal{O}_{K, \mathrm{sm}}$, we know that $i_{\mathrm{sm}}^{* \text { set }}\left(\mathcal{A}^{m}\right)$ is the special fiber $\mathcal{A}_{x}^{m}=\mathcal{A}^{m} \times_{\mathcal{O}_{K}} k$. Therefore $i_{\mathrm{sm}}^{*} M(\mathcal{A})=M\left(\mathcal{A}_{x}\right)=M\left(i^{*} \mathcal{A}\right)$ is Mac Lane's resolution of $\mathcal{A}_{x}$ in $\mathrm{Ab}\left(k_{\mathrm{sm}}\right)$, which is acyclic outside degree zero. Therefore $L_{n} i^{*} \mathcal{A}=0$ for any $n \geq 1$.

In the same way, we can prove that if $X$ is a scheme and $f$ is the continuous map from the big étale site of $X$ to the smooth site of $X$ defined by the identity, then $f^{*}$ admits a left derived functor $L f^{*}$, and we have $L_{n} f^{*} T=0$ for any smooth group scheme $T$ over $X$ and $n \geq 1$. This implies that [Mil06, Thm. 4.11] is true with the big étale site replaced by the smooth site by the same argument as above. This answers Milne's question made right after the cited theorem.

5.2. Bester-Bertapelle's isomorphism. First note that

$$
\operatorname{Ext}_{k_{\text {proet }}^{\text {indrat }}}^{1}(\boldsymbol{\Gamma}(A), \mathbb{Q} / \mathbb{Z})=\underset{n}{\lim } \operatorname{Ext}_{\mathrm{PAlg} / k}^{1}(\boldsymbol{\Gamma}(A), \mathbb{Z} / n \mathbb{Z})
$$

by (2.3.3) (b).

Proposition (5.2.1). Assume that $k$ is algebraically closed. The morphism

$$
\theta_{A}^{+1}(k): H^{1}\left(K, A^{\vee}\right) \rightarrow \operatorname{Ext}_{k_{\text {proet }}^{\text {indrat }}}^{1}(\boldsymbol{\Gamma}(A), \mathbb{Q} / \mathbb{Z})
$$


of (4.2.1) coincides with Bester-Bertapelle's isomorphism [Bes78, §2.7, Thm. 7.1], [Ber03, Thm. 3] when $K$ has equal characteristic and A has semistable reduction.

We prove this in this subsection. In [Bes78, §2.3, Thm. 3.1], Bester proved a duality theorem for cohomology of $\mathcal{O}_{K}$ with coefficients in finite flat group schemes. Based on this result, Bertapelle proved its generalization for coefficients in the quasifinite flat group scheme $\mathcal{A}[n]$ of torsion points of the Néron model of any semistable abelian variety $A$ ([Ber03, Thm. 1]). Then she deduced the existence of the above isomorphism for such $A$ ([Ber03, Thm. 2]), and deduced the existence of the above isomorphism for a general abelian variety.

In this subsection, we first give another proof of Bester's finite flat duality, by giving another construction of the duality morphism and directly showing that it is an isomorphism. The idea of proof is the same as Bester's (which is the local version of [AM76]), but we need to make it work within the formulation of this paper. Then we use our finite flat duality isomorphism (instead of Bester's isomorphism) as the input for Bertapelle's constructions. This outputs an isomorphism

$$
\psi_{A}: H^{1}\left(K, A^{\vee}\right)=\operatorname{Ext}_{k_{\text {proet }}^{\text {indrat }}}^{1}(\boldsymbol{\Gamma}(A), \mathbb{Q} / \mathbb{Z})
$$

as in the proof of Ber03, Thm. 2]. Finally we show that $\theta_{A}^{+1}(k)$ and $\psi_{A}$ are equal. Hence we do not actually compare our isomorphism and Bester's isomorphism (see (5.2.1.5) below for the reason). Therefore the statement of (5.2.1) is slightly imprecise in this sense. See (5.2.3.1) below for the precise statement. We do not have to compare Bertapelle's isomorphism with $\theta_{A}^{+1}(k)$ for non-semistable abelian varieties for the purpose of proving our main theorems. But this can be done; see (6.13) (b) below.

Throughout this subsection, we assume that $K$ has equal characteristic. Some statements can be formulated without assuming the residue field $k$ to be algebraically closed. Therefore we assume that $k$ is a general perfect field.

5.2.1. Bester's finite flat duality. Let $N$ be a finite flat group scheme over $\mathcal{O}_{K}$ with Cartier dual $N^{\mathrm{CD}}$. In this subsubsection, we first formulate a duality theorem that relates the two complexes

$$
R \boldsymbol{\Gamma}_{x}\left(\mathcal{O}_{K}, N^{\mathrm{CD}}\right), \quad R \boldsymbol{\Gamma}\left(\mathcal{O}_{K}, N\right)
$$

each other. This is written in Suz13, Rmk. 2.7.6] without proof. We show that this implies Bester's duality. The proof of the duality theorem itself will be given in the next subsubsection.

We have $R \boldsymbol{\Gamma}\left(K, \mathbf{G}_{m}\right)=\mathbf{K}^{\times}$as recalled in the previous section, and $R \boldsymbol{\Gamma}\left(\mathcal{O}_{K}, \mathbf{G}_{m}\right)=$ $\boldsymbol{\Gamma}\left(\mathcal{O}_{K}, \mathbf{G}_{m}\right)=\mathbf{U}_{K}$ by (3.4.2) (a) $)$. Hence we have

$$
R \boldsymbol{\Gamma}_{x}\left(\mathcal{O}_{K}, \mathbf{G}_{m}\right)=\mathbb{Z}[-1],
$$

which we call the trace isomorphism.

Let $N$ be a finite flat group scheme over $\mathcal{O}_{K}$ with Cartier dual $N^{\mathrm{CD}}$. Recall from (3.4.2) (b) and (3.4.6) that $R \boldsymbol{\Gamma}\left(\mathcal{O}_{K}, N\right) \in D^{b}\left(\mathrm{PAlg}_{\mathrm{uc}} / k\right), R \boldsymbol{\Gamma}_{x}\left(\mathcal{O}_{K}, N\right) \in$ $D^{b}\left(\operatorname{IAlg}_{\mathrm{uc}} / k\right)$ are P-acyclic and Serre reflexive, $\boldsymbol{\Gamma}\left(\mathcal{O}_{K}, N\right)$ is finite, $\mathbf{H}^{1}\left(\mathcal{O}_{K}, N\right)$ is connected pro-unipotent, $\mathbf{H}_{x}^{2}\left(\mathcal{O}_{K}, N\right) \in \operatorname{IAlg}_{\mathrm{uc}} / k$, and the cohomology of $R \boldsymbol{\Gamma}\left(\mathcal{O}_{K}, N\right)$ and $R \boldsymbol{\Gamma}_{x}\left(\mathcal{O}_{K}, N\right)$ vanishes at all other degrees. The morphism of functoriality of 
$R \tilde{\boldsymbol{\Gamma}}$ and $R \tilde{\boldsymbol{\Gamma}}_{x}$ in (3.3.8) and the trace isomorphism give morphisms

$$
\begin{aligned}
& R \boldsymbol{\Gamma}_{x}\left(\mathcal{O}_{K}, N^{\mathrm{CD}}\right) \rightarrow R \tilde{\boldsymbol{\Gamma}}_{x}\left(\mathcal{O}_{K}, R \mathbf{H o m}_{\mathcal{O}_{K}}\left(N, \mathbf{G}_{m}\right)\right) \\
& \quad \rightarrow R \mathbf{H o m}_{k_{\text {proat }}^{\text {indrat }}}\left(R \boldsymbol{\Gamma}\left(\mathcal{O}_{K}, N\right), \mathbb{Z}[-1]\right)=R \boldsymbol{\Gamma}\left(\mathcal{O}_{K}, N\right)^{\mathrm{SD}}[-1]
\end{aligned}
$$

Theorem (5.2.1.2). The morphism

$$
R \boldsymbol{\Gamma}_{x}\left(\mathcal{O}_{K}, N^{\mathrm{CD}}\right) \rightarrow R \boldsymbol{\Gamma}\left(\mathcal{O}_{K}, N\right)^{\mathrm{SD}}[-1]
$$

in $D\left(k_{\text {proet }}^{\text {indrat }}\right)$ defined above is an isomorphism.

This is stated in Suz13, Rmk. 2.7.6] without proof. We will prove this in the next subsubsection. We show here that this implies Bester's duality.

The both sides are in $D^{b}\left(\operatorname{IAlg}_{\mathrm{uc}} / k\right)$. The functor $R \Gamma\left(\bar{k}_{\text {proet }}, \cdot\right)$ restricted to $D^{b}\left(\operatorname{IAlg}_{\mathrm{uc}} / k\right)$ takes values in the derived category of torsion discrete $\operatorname{Gal}(\bar{k} / k)$ modules, since unipotent quasi-algebraic groups in positive characteristic are torsion. Applying this functor to the morphism in the theorem, we obtain a morphism

$$
R \Gamma_{x}\left(\mathcal{O}_{K}^{\mathrm{ur}}, N^{\mathrm{CD}}\right) \rightarrow R \operatorname{Hom}_{\overline{k_{\text {proet }}}}^{\text {indrat }}\left(R \boldsymbol{\Gamma}\left(\mathcal{O}_{K}, N\right), \mathbb{Q} / \mathbb{Z}\right)[-2]
$$

in the derived category of torsion discrete $\operatorname{Gal}(\bar{k} / k)$-modules, where $\mathcal{O}_{K}^{\text {ur }}$ is the maximal unramified extension of $\mathcal{O}_{K}$. We have

$$
\begin{aligned}
R \operatorname{Hom}_{\overline{\text { proet }}}^{\text {indrat }}\left(R \boldsymbol{\Gamma}\left(\mathcal{O}_{K}, N\right), \mathbb{Q} / \mathbb{Z}\right) & =R \operatorname{Hom}_{\mathrm{PAlg} / \bar{k}}\left(R \boldsymbol{\Gamma}\left(\mathcal{O}_{K}, N\right), \mathbb{Q} / \mathbb{Z}\right) \\
& \left(:=\underset{n}{\lim } R \operatorname{Hom}_{\mathrm{PAlg} / \bar{k}}\left(R \boldsymbol{\Gamma}\left(\mathcal{O}_{K}, N\right), \mathbb{Z} / n \mathbb{Z}\right)\right)
\end{aligned}
$$

by (2.3.3) (b). Let PFEt/ $k$ be the category of pro-finite-étale group schemes over $k$ and $\pi_{0}: \mathrm{PAlg} / k \rightarrow \mathrm{PFEt} / k$ the maximal pro-finite-étale quotient functor as before. We saw in 2.2 that PAlg/ $k$ (and also PFEt $/ k$ ) is the opposite of a Grothendieck category and hence has enough (K-)projectives. Hence $\pi_{0}$ admits a left derived functor $L \pi_{0}: D(\mathrm{PAlg} / k) \rightarrow D(\mathrm{PFEt} / k)$. The $n$-th functor $\pi_{n}:=L_{n} \pi_{0}$ is the $n$ th homotopy group functor defined by Serre Ser60, §5.3]. The functor $\pi_{0}$ is left adjoint to the inclusion functor PFEt $/ k \hookrightarrow \mathrm{PAlg} / k$, which is exact. Hence $\pi_{0}$ sends projectives to projectives. By [KS06, Prop. 13.3.13 (ii)], we have

$$
R \operatorname{Hom}_{\mathrm{PAlg} / \bar{k}}\left(R \boldsymbol{\Gamma}\left(\mathcal{O}_{K}, N\right), \mathbb{Q} / \mathbb{Z}\right)=R \operatorname{Hom}_{\mathrm{PFEt} / \bar{k}}\left(L \pi_{0} R \boldsymbol{\Gamma}\left(\mathcal{O}_{K}, N\right), \mathbb{Q} / \mathbb{Z}\right) .
$$

We have a distinguished triangle

$$
L \pi_{0} \boldsymbol{\Gamma}\left(\mathcal{O}_{K}, N\right) \rightarrow L \pi_{0} R \boldsymbol{\Gamma}\left(\mathcal{O}_{K}, N\right) \rightarrow L \pi_{0} \mathbf{H}^{1}\left(\mathcal{O}_{K}, N\right)[-1] .
$$

Recall from Ser60, $\S 10.2$, Thm. 2] that $\pi_{n}=L_{n} \pi_{0}=0$ for all $n \geq 2$. The finiteness of $\boldsymbol{\Gamma}\left(\mathcal{O}_{K}, N\right)$ implies that $\pi_{1} \boldsymbol{\Gamma}\left(\mathcal{O}_{K}, N\right)=0$ and hence $L \pi_{0} \boldsymbol{\Gamma}\left(\mathcal{O}_{K}, N\right)=\boldsymbol{\Gamma}\left(\mathcal{O}_{K}, N\right)$. The connectedness of $\mathbf{H}^{1}\left(\mathcal{O}_{K}, N\right)$ implies that $L \pi_{0} \mathbf{H}^{1}\left(\mathcal{O}_{K}, N\right)=\pi_{1} \mathbf{H}^{1}\left(\mathcal{O}_{K}, N\right)[1]$. Therefore $L \pi_{0} R \boldsymbol{\Gamma}\left(\mathcal{O}_{K}, N\right)$ is concentrated in degree 0 and the above triangle reduces to an exact sequence

$$
0 \rightarrow \boldsymbol{\Gamma}\left(\mathcal{O}_{K}, N\right) \rightarrow H^{0} L \pi_{0} R \boldsymbol{\Gamma}\left(\mathcal{O}_{K}, N\right) \rightarrow \pi_{1} \mathbf{H}^{1}\left(\mathcal{O}_{K}, N\right) \rightarrow 0 .
$$

As in the proof of (3.4.2) (b), let $0 \rightarrow N \rightarrow G \rightarrow H \rightarrow 0$ be an exact sequence of group schemes over $\mathcal{O}_{K}$ with $G, H$ smooth affine with connected fibers. Then (3.4.2) (a) shows that $R \boldsymbol{\Gamma}\left(\mathcal{O}_{K}, G\right)$ is concentrated in degree zero and $\boldsymbol{\Gamma}\left(\mathcal{O}_{K}, G\right) \in \mathrm{PAlg} / k$ is connected. Hence $L \pi_{0} R \boldsymbol{\Gamma}\left(\mathcal{O}_{K}, G\right)=\pi_{1} \boldsymbol{\Gamma}\left(\mathcal{O}_{K}, G\right)[1]$ and $L \pi_{0} R \boldsymbol{\Gamma}\left(\mathcal{O}_{K}, H\right)=$ $\pi_{1} \boldsymbol{\Gamma}\left(\mathcal{O}_{K}, H\right)[1]$. Therefore the distinguished triangle

$$
L \pi_{0} R \boldsymbol{\Gamma}\left(\mathcal{O}_{K}, N\right) \rightarrow L \pi_{0} \boldsymbol{\Gamma}\left(\mathcal{O}_{K}, G\right) \rightarrow L \pi_{0} \boldsymbol{\Gamma}\left(\mathcal{O}_{K}, H\right)
$$


is reduced to an exact sequence

$$
0 \rightarrow \pi_{1} \boldsymbol{\Gamma}\left(\mathcal{O}_{K}, G\right) \rightarrow \pi_{1} \boldsymbol{\Gamma}\left(\mathcal{O}_{K}, H\right) \rightarrow H^{0} L \pi_{0} R \boldsymbol{\Gamma}\left(\mathcal{O}_{K}, N\right) \rightarrow 0 .
$$

This description shows the following intermediate result:

Proposition (5.2.1.3). The complex $L \pi_{0} R \boldsymbol{\Gamma}\left(\mathcal{O}_{K}, N\right)$ is concentrated in degree 0 whose cohomology is canonically identified with Bester's group $\mathscr{F}(N)$ [Bes78, §1.3].

Therefore our morphism after $R \Gamma\left(\bar{k}_{\text {proet }}, \cdot\right)$ takes the form

$$
R \Gamma_{x}\left(\mathcal{O}_{K}^{\mathrm{ur}}, N^{\mathrm{CD}}\right) \rightarrow \operatorname{Hom}_{\mathrm{PFEt} / \bar{k}}(\mathscr{F}(N), \mathbb{Q} / \mathbb{Z})[-2] .
$$

Recall again that the left-hand side is concentrated in degree 2.

Theorem (5.2.1.4). The morphism

$$
H_{x}^{2}\left(\mathcal{O}_{K}^{\mathrm{ur}}, N^{\mathrm{CD}}\right) \rightarrow \mathscr{F}(N)^{\mathrm{PD}}
$$

of torsion discrete $\operatorname{Gal}(\bar{k} / k)$-modules thus obtained is an isomorphism.

This is stated in Bes78, $\S 2.3$, Thm. 3.1]. We will directly prove this by proving (5.2.1.2) in the next subsubsection.

Remark (5.2.1.5). Comparing our finite flat duality isomorphism above and Bester's isomorphism, which we omit here, is highly technical and complicated. We have to bring many constructions in Bester's work into derived categories of sheaves. Several technical fixes that we do not discuss here in detail are also necessary. In this paper, we avoid the comparison and fixes to save pages and prove Bester's duality independently.

Below we merely indicate one issue that needs a fix. Assume for simplicity that the residue field $k$ is algebraically closed. Recall that the finite flat site $\operatorname{Spec} \mathcal{O}_{K \text {,ffl }}$ of $\mathcal{O}_{K}$ is the category of finite flat $\mathcal{O}_{K}$-algebras where a cover of an object $S$ is a finite family $\left\{S_{i}\right\}$ of finite flat $S$-algebras such that $S \rightarrow \prod S_{i}$ is faithfully flat. For a finite flat group scheme $N$ over $\mathcal{O}_{K}$, Bester defined his group $\mathscr{F}(N)$ in Bes78, $\S 1.3$, Def. 3.3] not only as a pro-finite-étale group over $k$ but also as a sheaf on the finite flat site $\operatorname{Spec} \mathcal{O}_{K \text {,ffl }}$. Let us denote this sheaf by $\mathscr{F}_{\operatorname{sh}}(N)$ for clarity. He stated the existence of a trace isomorphism

$$
H_{x}^{2}\left(\mathcal{O}_{K}, \mathscr{F}_{\operatorname{sh}}\left(\mu_{p^{n}}\right)\right)=\mathbb{Z} / p^{n} \mathbb{Z}
$$

for any $n \geq 1$ in [Bes78, $\S 2.6$, Lem. 6.2] and its proof. Here $\mathscr{F}_{\operatorname{sh}}\left(\mu_{p^{n}}\right)$ is pulled back to $\operatorname{Spec} \mathcal{O}_{K \text {,fppf }}([\operatorname{Bes} 78, \S 1.2$, Rmk. 2.2] $)$ and then taken the fppf cohomology with support $([\operatorname{Bes78}, \S 2.2])$. However, we can show that $\mathscr{F}_{\operatorname{sh}}\left(\mu_{p^{n}}\right)=0$ as a sheaf on $\operatorname{Spec} \mathcal{O}_{K, \mathrm{ff}}$, which contradicts to his statement.

To prove $\mathscr{F}_{\mathrm{sh}}\left(\mu_{p^{n}}\right)=0$, we first recall the definition of this sheaf. For a flat group scheme $G$ over $\mathcal{O}_{K}$, recall from [Bes78, $\left.\left.\S 1.2, \mathrm{i}\right)\right]$ that another sheaf denoted by $\pi_{1}(\tilde{G})$ on $\operatorname{Spec} \mathcal{O}_{K \text {,ff }}$ is defined as follows. Assume for simplicity that $G$ is affine. For a finite flat local $\mathcal{O}_{K}$-algebra $S$, let $\operatorname{Res}_{S / \mathcal{O}_{K}}$ be the Weil restriction functor. Consider the Greenberg transform $\Gamma\left(\mathcal{O}_{K}, \operatorname{Res}_{S / \mathcal{O}_{K}} G\right)$ of $\operatorname{Res}_{S / \mathcal{O}_{K}} G$ and denote it by $\boldsymbol{\Gamma}(S, G)$. This is a proalgebraic group over $k$ representing the functor

$$
R \mapsto \Gamma\left(R[[T]], \operatorname{Res}_{S / \mathcal{O}_{K}} G\right)=\Gamma\left(R[[T]] \otimes_{k[[T]]} S, G\right)
$$

on the category of $k$-algebras, where we identified $\mathcal{O}_{K}=k[[T]]$. We have

$$
\Gamma(S, G)=\underset{n}{\lim } \operatorname{Res}_{\left(S / T^{n} S\right) / k} G
$$


Define $\pi_{1}(\tilde{G})$ to be the presheaf that assigns to each a finite flat $\mathcal{O}_{K}$-algebra $S$ and its decomposition $\prod S_{i}$ into local rings the group

$$
\prod_{i} \pi_{1} \Gamma\left(S_{i}, G\right) .
$$

This is in fact a sheaf by the faithfully flat descent and the left exactness of $\pi_{1}$ ([Bes78, §1.2, Lem. 2.1]). Here we treat $\pi_{1}(\tilde{G})$ as a single set of notation and do not decompose it into the $\pi_{1}$ of anything in the usual sense. Let $N$ be a finite flat group scheme over $\mathcal{O}_{K}$. Take an exact sequence $0 \rightarrow N \rightarrow G \rightarrow H \rightarrow 0$ of group schemes over $\mathcal{O}_{K}$ with $G, H$ smooth affine with connected fibers, as we took in the proof of (3.4.2) (b). Then the sheaf $\mathscr{F}_{\mathrm{sh}}(N) \in \mathrm{Ab}\left(\mathcal{O}_{K, \mathrm{ffl}}\right)$, as defined in Bes78, $\S 1.3$, Def. 3.3], is the cokernel of the morphism of sheaves

$$
\pi_{1}(\tilde{G}) \rightarrow \pi_{1}(\tilde{H}) \quad \text { in } \quad \operatorname{Ab}\left(\mathcal{O}_{K, f f}\right) .
$$

We show that $\mathscr{F}_{\operatorname{sh}}\left(\mu_{p^{n}}\right)=0$ for any $n \geq 1$ as a sheaf on $\operatorname{Spec} \mathcal{O}_{K, \text { fft }}$. For this, it is enough to show that for any finite flat local $\mathcal{O}_{K}$-algebra $S$, there exists a finite faithfully flat local $S$-algebra $S^{\prime}$ such that the homomorphism from the cokernel of

$$
\pi_{1} \boldsymbol{\Gamma}\left(S, \mathbf{G}_{m}\right) \stackrel{p^{n}}{\rightarrow} \pi_{1} \boldsymbol{\Gamma}\left(S, \mathbf{G}_{m}\right)
$$

to the cokernel of

$$
\pi_{1} \boldsymbol{\Gamma}\left(S^{\prime}, \mathbf{G}_{m}\right) \stackrel{p^{n}}{\rightarrow} \pi_{1} \boldsymbol{\Gamma}\left(S^{\prime}, \mathbf{G}_{m}\right)
$$

is zero. The group $\boldsymbol{\Gamma}\left(S, \mathbf{G}_{m}\right)$ is connected. Since $\operatorname{Res}_{R / k} \mu_{p^{n}}$ is connected for any finite $k$-algebra $R 14$ it follows that $\boldsymbol{\Gamma}\left(S, \mu_{p^{n}}\right)$ is also connected (cf. Bes78, $\S 1.1$, Lem. 1.2]). This implies

$$
\left(\pi_{1} \boldsymbol{\Gamma}\left(S, \mathbf{G}_{m}\right)\right) / p^{n}=\pi_{1}\left(\boldsymbol{\Gamma}\left(S, \mathbf{G}_{m}\right) / p^{n}\right),
$$

where $/ p^{n}$ denotes the cokernel of multiplication by $p^{n}$. Similar for $\boldsymbol{\Gamma}\left(S^{\prime}, \cdot\right)$. Hence it is enough to show that the morphism $\boldsymbol{\Gamma}\left(S, \mathbf{G}_{m}\right) \rightarrow \boldsymbol{\Gamma}\left(S^{\prime}, \mathbf{G}_{m}\right)$ factors through the image $D$ of the $p^{n}$-th power map $\boldsymbol{\Gamma}\left(S^{\prime}, \mathbf{G}_{m}\right) \rightarrow \boldsymbol{\Gamma}\left(S^{\prime}, \mathbf{G}_{m}\right)$. The groups $\boldsymbol{\Gamma}\left(S, \mathbf{G}_{m}\right)$, $\boldsymbol{\Gamma}\left(S^{\prime}, \mathbf{G}_{m}\right)$ and $D$ are inverse limits of smooth algebraic groups over $k$. Therefore it is enough to check the statement for $k$-points ( $k$ being algebraically closed). The requirement for $S^{\prime}$ is now that every element of $\Gamma\left(S, \mathbf{G}_{m}\right)$ should become a $p^{n_{-}}$ th power in $\Gamma\left(S^{\prime}, \mathbf{G}_{m}\right)$. If $S=k[[T]]\left[x_{1}, \ldots, x_{n}\right] /\left(f_{1}, \ldots, f_{m}\right)$ for some polynomials $f_{1}, \ldots, f_{m}$ of the polynomial ring, then $S^{\prime}=k\left[\left[T^{1 / p^{n}}\right]\right]\left[x_{1}^{1 / p^{n}}, \ldots, x_{n}^{1 / p^{n}}\right] /\left(f_{1}, \ldots, f_{m}\right)$ does the job. This choice of $S^{\prime}$ was suggested by Bertapelle.

We should instead consider the mapping cone of the morphism (5.2.1.6) in $D\left(\mathcal{O}_{K, \text { ffl }}\right)$. This is the first step for a fix, but we should stop here.

5.2.2. Proof of the finite flat duality. Let $N$ be a finite flat group scheme over $K$. The morphism of functoriality of $R \tilde{\boldsymbol{\Gamma}}$ (3.3.8) and the trace morphism (4.1.1) give morphisms

$$
\begin{aligned}
& R \boldsymbol{\Gamma}\left(K, N^{\mathrm{CD}}\right) \rightarrow R \tilde{\boldsymbol{\Gamma}}\left(K, R \mathbf{H o m}_{K}\left(N, \mathbf{G}_{m}\right)\right) \\
& \quad \rightarrow R \mathbf{H o m}_{k_{\text {proet }}^{\text {indrat }}}(R \boldsymbol{\Gamma}(K, N), \mathbb{Z})=R \boldsymbol{\Gamma}(K, N)^{\mathrm{SD}}
\end{aligned}
$$

\footnotetext{
14 To see this, it is enough to show that the scheme-theoretically isomorphic group $\operatorname{Res}_{R / k} \alpha_{p^{n}}$ is connected. Let $\left\{r_{i}\right\}_{i=1}^{d}$ be a $k$-basis of $R$. Write $r_{j}^{p^{n}}=\sum_{i} c_{i j} r_{i}$ with $c_{i j} \in k$. Let $C: \mathbf{G}_{a}^{d} \rightarrow \mathbf{G}_{a}^{d}$ be the $k$-linear morphism given by the matrix with entries $c_{i j}$. Then $\operatorname{Res}_{R / k} \alpha_{p^{n}}=\operatorname{Ker}\left(C F^{n}\right)$ as a subgroup of $\mathbf{G}_{a}^{d}=\operatorname{Res}_{R / k} \mathbf{G}_{a}$, where $F: \mathbf{G}_{a}^{d} \rightarrow \mathbf{G}_{a}^{d}$ is the Frobenius over $k$. Hence $\operatorname{Res}_{R / k} \alpha_{p^{n}}$ is an extension of the vector group $\operatorname{Ker} C$ by $\alpha_{p^{n}}^{d}$, thus connected.
} 
Theorem (5.2.2.1). The morphism

$$
R \boldsymbol{\Gamma}\left(K, N^{\mathrm{CD}}\right) \rightarrow R \boldsymbol{\Gamma}(K, N)^{\mathrm{SD}}
$$

in $D\left(k_{\text {proet }}^{\text {indrat }}\right)$ defined above is an isomorphism.

This is proved in Suz13, Thm. 2.7.1] for the case $N$ does not have connected unipotent part. We prove (5.2.2.1) for general $N$, in addition to and at the same time proving (5.2.1.2). Note that Pépin Pép14, \$7.6.1] also proved a result equivalent to (5.2.2.1) for general $N$.

Proposition (5.2.2.2). For a finite flat group scheme $N$ over $\mathcal{O}_{K}$ with Cartier dual $N^{\mathrm{CD}}$, we have a morphism of distinguished triangles

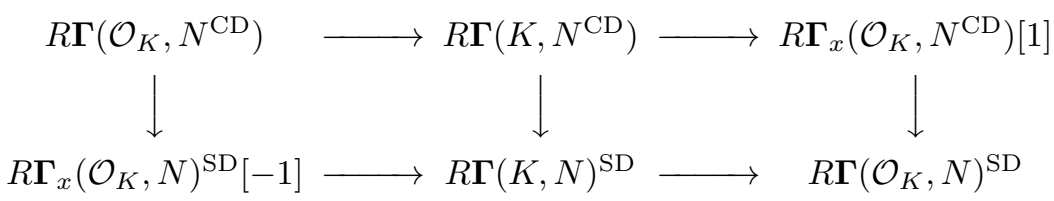

in $D\left(k_{\text {proet }}^{\text {indrat }}\right)$.

Proof. Apply the diagram in (3.3.8) for $A=N$ and $B=\mathbf{G}_{m}$ and note that the trace (iso)morphisms $R \boldsymbol{\Gamma}\left(K, \mathbf{G}_{m}\right) \rightarrow \mathbb{Z}$ and $R \boldsymbol{\Gamma}_{x}\left(\mathcal{O}_{K}, \mathbf{G}_{m}\right)[1] \rightarrow \mathbb{Z}$ are compatible with the morphism $R \boldsymbol{\Gamma}\left(K, \mathbf{G}_{m}\right) \rightarrow R \boldsymbol{\Gamma}_{x}\left(\mathcal{O}_{K}, \mathbf{G}_{m}\right)[1]$ by construction.

Proposition (5.2.2.3). To prove (5.2.1.2) and (5.2.2.1), it is enough to show (5.2.1.2) for the case that the finite flat group scheme $N$ or $N^{\mathrm{CD}}$ has height 1, i.e. has zero Frobenius.

Proof. First note that if $0 \rightarrow N_{1} \rightarrow N_{2} \rightarrow N_{3} \rightarrow 0$ is an exact sequence of finite flat group schemes (over $\mathcal{O}_{K}$ or $K$ ) and if the statements are true for any two of them, then so is for the other by the five lemma. The statements are classical and elementary if $N$ has order prime to $p$ (cf. [Ber03, §3.2]). Assume $N$ (over $\mathcal{O}_{K}$ or $K)$ has $p$-power order. Then it has a filtration whose successive subquotients are either height 1 or have Cartier dual of height 1 ([Bes78, §2.6, Lem. 6.1]). A finite flat group scheme over $K$ of height 1 can be extended to a finite flat group scheme over $\mathcal{O}_{K}$ of height 1 by [Mil06, III, Prop. B.4].

Let $\Omega_{\mathcal{O}_{K}}^{1}=\Omega_{\mathcal{O}_{K} / k}^{1}$ and $\Omega_{K}^{1}=\Omega_{K / k}^{1}$ be the first differential modules. We identify them as group schemes isomorphic to $\mathbf{G}_{a}$. We have $R \boldsymbol{\Gamma}\left(\mathcal{O}_{K}, \mathbf{G}_{a}\right)=\mathbf{O}_{K}$, $R \boldsymbol{\Gamma}\left(K, \mathbf{G}_{a}\right)=\mathbf{K}$, and hence $R \boldsymbol{\Gamma}_{x}\left(\mathcal{O}_{K}, \mathbf{G}_{a}\right)=\mathbf{K} / \mathbf{O}_{K}[-1]$. The residue map $\sum a_{n} T^{n} d T / T \mapsto a_{0}$ may be viewed as a morphism

$$
\operatorname{Res}: R \boldsymbol{\Gamma}\left(K, \Omega_{K}^{1}\right) \rightarrow R \boldsymbol{\Gamma}_{x}\left(\mathcal{O}_{K}, \Omega_{\mathcal{O}_{K}}^{1}\right)[1] \rightarrow \mathbf{G}_{a}
$$

in $D\left(k_{\text {proet }}^{\text {indrat }}\right)$. Together with the coboundary map $\mathbf{G}_{a} \rightarrow \mathbb{Z} / p \mathbb{Z}[1]$ of the ArtinSchreier sequence $0 \rightarrow \mathbb{Z} / p \mathbb{Z} \rightarrow \mathbf{G}_{a} \rightarrow \mathbf{G}_{a} \rightarrow 0$ and the coboundary map $\mathbb{Z} / p \mathbb{Z}[1] \rightarrow$ $\mathbb{Z}[2]$ of the sequence $0 \rightarrow \mathbb{Z} \rightarrow \mathbb{Z} \rightarrow \mathbb{Z} / p \mathbb{Z} \rightarrow 0$, we have morphisms

$$
R \boldsymbol{\Gamma}\left(K, \Omega_{K}^{1}\right) \rightarrow R \boldsymbol{\Gamma}_{x}\left(\mathcal{O}_{K}, \Omega_{\mathcal{O}_{K}}^{1}\right)[1] \rightarrow \mathbb{Z}[2]
$$

in $D\left(k_{\text {proet }}^{\text {indrat }}\right)$, which we call the additive trace morphisms (as opposed to the multiplicative trace morphisms $\left.R \boldsymbol{\Gamma}\left(K, \mathbf{G}_{m}\right) \rightarrow R \boldsymbol{\Gamma}_{x}\left(\mathcal{O}_{K}, \mathbf{G}_{m}\right)[1]=\mathbb{Z}\right)$. 
Proposition (5.2.2.4). Let $M \times L \rightarrow \Omega_{\mathcal{O}_{K}}^{1}$ (resp. $M \times L \rightarrow \Omega_{K}^{1}$ ) be a perfect pairing of finite free $\mathcal{O}_{K}$-modules (resp. finite free $K$-modules). Consider the morphisms

$$
\begin{aligned}
R \boldsymbol{\Gamma}_{x}\left(\mathcal{O}_{K}, M\right) & \rightarrow R \boldsymbol{\Gamma}_{x}\left(\mathcal{O}_{K}, R \mathbf{H o m}_{\mathcal{O}_{K}}\left(L, \Omega_{\mathcal{O}_{K}}^{1}\right)\right) \\
& \rightarrow R \mathbf{H o m}_{k_{\text {proet }}^{\text {indrat }}}\left(R \boldsymbol{\Gamma}\left(\mathcal{O}_{K}, L\right), R \boldsymbol{\Gamma}_{x}\left(\mathcal{O}_{K}, \Omega_{\mathcal{O}_{K}}^{1}\right)\right) \\
& \rightarrow R \boldsymbol{\Gamma}\left(\mathcal{O}_{K}, L\right)^{\mathrm{SD}}[1]
\end{aligned}
$$

and similarly

$$
R \boldsymbol{\Gamma}\left(\mathcal{O}_{K}, M\right) \rightarrow R \boldsymbol{\Gamma}_{x}\left(\mathcal{O}_{K}, L\right)^{\mathrm{SD}}[1]
$$

(resp.

$$
\begin{aligned}
R \boldsymbol{\Gamma}(K, M) & \rightarrow R \boldsymbol{\Gamma}\left(K, R \operatorname{Hom}_{K}\left(L, \Omega_{K}^{1}\right)\right) \\
& \rightarrow R \mathbf{H o m}_{k_{\text {proet }}^{\text {indrat }}}\left(R \boldsymbol{\Gamma}(K, L), R \boldsymbol{\Gamma}\left(K, \Omega_{K}^{1}\right)\right) \\
& \left.\rightarrow R \boldsymbol{\Gamma}(K, L)^{\mathrm{SD}}[2]\right)
\end{aligned}
$$

in $D\left(k_{\text {proet }}^{\text {indrat }}\right)$ defined by the functoriality of $R \tilde{\boldsymbol{\Gamma}}_{x}\left(\mathcal{O}_{K}, \cdot\right)($ resp. $R \tilde{\boldsymbol{\Gamma}}(K, \cdot))$ and the additive trace morphism. These are isomorphisms.

Proof. The latter isomorphism (for cohomology of $K$ ) follows from the former two isomorphisms (for cohomology of $\mathcal{O}_{K}$ ) by a morphism of distinguished triangles similar to the one in (5.2.2.2). For the former two, it is enough to treat the morphism $R \boldsymbol{\Gamma}_{x}\left(\mathcal{O}_{K}, M\right) \rightarrow R \boldsymbol{\Gamma}\left(\mathcal{O}_{K}, L\right)^{\mathrm{SD}}[1]$ since $\mathbf{O}_{K} \cong \mathbf{G}_{a}^{\mathbb{N}}, \mathbf{K} / \mathbf{O}_{K} \cong \mathbf{G}_{a}^{\oplus \mathbb{N}}$ and $\mathbf{K} \cong$ $\mathbf{G}_{a}^{\mathbb{N}} \oplus \mathbf{G}_{a}^{\oplus \mathbb{N}}$ are all Serre reflexive by (2.4.1) (b). We may assume that $M=\mathcal{O}_{K}$, $L=\Omega_{\mathcal{O}_{K}}^{1}$ and the pairing $M \times L \rightarrow \Omega_{\mathcal{O}_{K}}^{1}$ is the multiplication. Then the morphism can be written as the composite

$$
\mathbf{K} / \mathbf{O}_{K} \rightarrow \mathbf{H o m}_{k_{\text {proet }}^{\text {indrat }}}\left(\boldsymbol{\Gamma}\left(\mathcal{O}_{K}, \Omega_{\mathcal{O}_{K}}^{1}\right), \mathbf{G}_{a}\right) \rightarrow \boldsymbol{\Gamma}\left(\mathcal{O}_{K}, \Omega_{\mathcal{O}_{K}}^{1}\right)^{\mathrm{SD}}[2],
$$

where the first morphism comes from the $k$-linear map $K / \mathcal{O}_{K} \rightarrow \operatorname{Hom}_{k \text {-mod }}\left(\Omega_{\mathcal{O}_{K}}^{1}, k\right)$, the residue map. We have

$$
\mathbf{H o m}_{k_{\text {proet }}^{\text {indrat }}}\left(\boldsymbol{\Gamma}\left(\mathcal{O}_{K}, \Omega_{\mathcal{O}_{K}}^{1}\right), \mathbf{G}_{a}\right)=\underset{n \geq 1}{\lim } \operatorname{Hom}_{k_{\mathrm{proet}}^{\text {indrat }}}\left(\boldsymbol{\Gamma}\left(\mathcal{O}_{K} / \mathfrak{p}_{K}^{n}, \Omega_{\mathcal{O}_{K}}^{1}\right), \mathbf{G}_{a}\right)
$$

by (2.3.3) (ㄷ) and (3.1.3) (d). Hence the mentioned morphism can also be written as the direct limit in $n \geq 1$ of the morphisms

$$
\mathbf{p}_{K}^{-n} / \mathbf{O}_{K} \rightarrow \mathbf{H o m}_{k_{\text {proet }}^{\text {ind rat }}}\left(\boldsymbol{\Gamma}\left(\mathcal{O}_{K} / \mathfrak{p}_{K}^{n}, \Omega_{\mathcal{O}_{K}}^{1}\right), \mathbf{G}_{a}\right),
$$

where $\mathbf{p}_{K}^{-n}\left(k^{\prime}\right)=\mathfrak{p}_{K}^{-n} \otimes_{\mathcal{O}_{K}} \mathbf{O}_{K}\left(k^{\prime}\right)$ for $k^{\prime} \in k^{\text {indrat }}$. For each $n$, this morphism comes from the $k$-linear morphism

$$
\operatorname{Res}: \mathfrak{p}_{K}^{-n} / \mathcal{O}_{K} \rightarrow \operatorname{Hom}_{k \text {-mod }}\left(\Gamma\left(\mathcal{O}_{K} / \mathfrak{p}_{K}^{n}, \Omega_{\mathcal{O}_{K}}^{1}\right), k\right),
$$

which is well-known (and easily seen) to be an isomorphism. Hence the Breen-Serre duality [Mil06, III, Lem. 0.13 (c)] (where the perfect étale site is used) and (2.4.1) (b) shows that

$$
\mathbf{p}_{K}^{-n} / \mathbf{O}_{K} \stackrel{\sim}{\rightarrow} \boldsymbol{\Gamma}\left(\mathcal{O}_{K} / \mathfrak{p}_{K}^{n}, \Omega_{\mathcal{O}_{K}}^{1}\right){ }^{\mathrm{SD}}[2] .
$$

The direct limit in $n$ gives the result.

We denote $K^{\prime}=K^{1 / p}$ and $\mathcal{O}_{K}^{\prime}=\mathcal{O}_{K}^{1 / p}$, which are viewed as a $K$-algebra and an $\mathcal{O}_{K}$-algebra, respectively, via inclusions $K \hookrightarrow K^{\prime}$ and $\mathcal{O}_{K} \hookrightarrow \mathcal{O}_{K}^{\prime}$. Let $F: \operatorname{Spec} K^{\prime} \rightarrow \operatorname{Spec} K$ and $F: \operatorname{Spec} \mathcal{O}_{K}^{\prime} \rightarrow \operatorname{Spec} \mathcal{O}_{K}$ be the natural morphisms. 
Let $N$ be a finite flat group scheme over $\mathcal{O}_{K}$ of height 1. Recall from [AM76, Prop. 1.1, Lem. 2.2] that there exist canonical exact sequences

$$
\begin{gathered}
0 \rightarrow N^{\mathrm{CD}} \rightarrow V^{0}\left(N^{\mathrm{CD}}\right) \rightarrow V^{1}\left(N^{\mathrm{CD}}\right) \rightarrow 0, \\
0 \rightarrow N \rightarrow F_{*} N \rightarrow U^{0}(N) \rightarrow U^{1}(N) \rightarrow 0
\end{gathered}
$$

of group schemes over $\mathcal{O}_{K}$. The terms $U^{i}(N), V^{i}\left(N^{\mathrm{CD}}\right)$ are vector groups. The second exact sequence above for $N=\mu_{p}$ is explicitly given by

$$
0 \rightarrow \mu_{p} \rightarrow F_{*} \mu_{p} \rightarrow F_{*} \Omega_{\mathcal{O}_{K}^{\prime}}^{1} \rightarrow \Omega_{\mathcal{O}_{K}}^{1} \rightarrow 0,
$$

where the middle morphism is $F_{*}$ dlog and the right morphism is the $\mathcal{O}$-linear Cartier operator $C$ minus the formal $p$-th power $W^{*}$ (AM76, Lem. 2.1, 2.2]). Let $U(N)$ be the complex $U^{0}(N) \rightarrow U^{1}(N)$ in degrees 0 and 1 and $V\left(N^{\mathrm{CD}}\right)$ the complex $V^{0}\left(N^{\mathrm{CD}}\right) \rightarrow V^{1}\left(N^{\mathrm{CD}}\right)$ in degrees 0 and 1. The morphism $U(N) \rightarrow N[1]$ induces isomorphisms

$$
\begin{aligned}
R \Gamma\left(\mathbf{O}_{K}\left(k^{\prime}\right), U(N)\right) & \stackrel{\sim}{\rightarrow} R \Gamma\left(\mathbf{O}_{K}\left(k^{\prime}\right), N\right)[1], \\
R \Gamma\left(\mathbf{K}\left(k^{\prime}\right), U(N)\right) & \stackrel{\sim}{\rightarrow} R \Gamma\left(\mathbf{K}\left(k^{\prime}\right), N\right)[1]
\end{aligned}
$$

for any $k^{\prime} \in k^{\text {indrat }}$ by AM76, Prop. 2.4]. (One checks that the proof there also works for our situation $\operatorname{Spec} \mathbf{O}_{K}\left(k^{\prime}\right) \rightarrow \operatorname{Spec} k^{\prime}$ and $\operatorname{Spec} \mathbf{K}\left(k^{\prime}\right) \rightarrow \operatorname{Spec} k^{\prime}$.) Therefore we have

$$
\begin{aligned}
R \boldsymbol{\Gamma}\left(\mathcal{O}_{K}, U(N)\right) & \stackrel{\sim}{\rightarrow} R \boldsymbol{\Gamma}\left(\mathcal{O}_{K}, N\right)[1], \\
R \boldsymbol{\Gamma}(K, U(N)) & \stackrel{\sim}{\rightarrow} R \boldsymbol{\Gamma}(K, N)[1], \\
R \boldsymbol{\Gamma}_{x}\left(\mathcal{O}_{K}, U(N)\right) & \stackrel{\sim}{\rightarrow} R \boldsymbol{\Gamma}_{x}\left(\mathcal{O}_{K}, N\right)[1] .
\end{aligned}
$$

Also

$$
\begin{aligned}
R \boldsymbol{\Gamma}\left(\mathcal{O}_{K}, V\left(N^{\mathrm{CD}}\right)\right) & \cong R \boldsymbol{\Gamma}\left(\mathcal{O}_{K}, N^{\mathrm{CD}}\right), \\
R \boldsymbol{\Gamma}\left(K, V\left(N^{\mathrm{CD}}\right)\right) & \cong R \boldsymbol{\Gamma}\left(K, N^{\mathrm{CD}}\right), \\
R \boldsymbol{\Gamma}_{x}\left(\mathcal{O}_{K}, V\left(N^{\mathrm{CD}}\right)\right) & \cong R \boldsymbol{\Gamma}_{x}\left(\mathcal{O}_{K}, N^{\mathrm{CD}}\right) .
\end{aligned}
$$

By [AM76, Prop. 3.4], there exists a canonical pairing

$$
V\left(N^{\mathrm{CD}}\right) \times U(N) \rightarrow U\left(\mu_{p}\right)
$$

of complexes of group schemes over $\mathcal{O}_{K}$. The parts

$$
V^{1}\left(N^{\mathrm{CD}}\right) \times U^{0}(N) \rightarrow U^{1}\left(\mu_{p}\right)=\Omega_{\mathcal{O}_{K}}^{1}, \quad V^{0}\left(N^{\mathrm{CD}}\right) \times U^{1}(N) \rightarrow U^{1}\left(\mu_{p}\right)=\Omega_{\mathcal{O}_{K}}^{1}
$$

are given by perfect pairings of finite free $\mathcal{O}_{K}$-modules. Let $Z(N)$ be the complex $F_{*} N \rightarrow U^{0}(N) \rightarrow U^{1}(N)$ of group schemes in degrees $0,1,2$, which is a resolution of $N$. By [AM76, (4.7)], the diagram

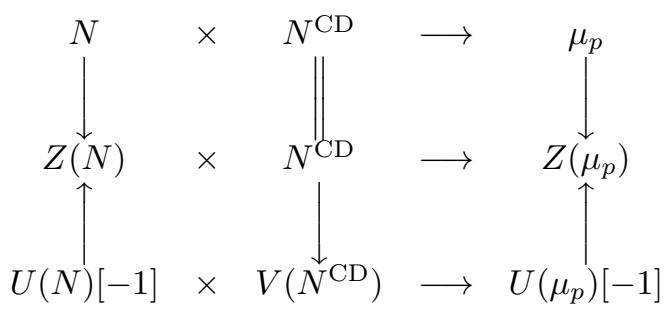

of pairings of complexes of group schemes over $\mathcal{O}_{K}$ is commutative. 
The complete discrete valuation field $K^{\prime}=K^{1 / p}$ has residue field $k$. We can define a sheaf of rings $\mathbf{K}^{\prime}=\boldsymbol{\Gamma}\left(K^{\prime}, \mathbf{G}_{a}\right)=\boldsymbol{\Gamma}\left(K, F_{*} \mathbf{G}_{a}\right)$ on Spec $k_{\text {proet }}^{\text {indrat }}$ in a way similar to $\mathbf{K}$. We also have a residue map $\boldsymbol{\Gamma}\left(K, F_{*} \Omega_{K^{\prime}}^{1}\right)=\boldsymbol{\Gamma}\left(K^{\prime}, \Omega_{K^{\prime}}^{1}\right) \rightarrow \mathbf{G}_{a}$ for $K^{\prime}$.

Proposition (5.2.2.8). The residue map gives a morphism of complexes from

$$
R \boldsymbol{\Gamma}_{x}\left(\mathcal{O}_{K}, U\left(\mu_{p}\right)\right)=\left[\frac{\boldsymbol{\Gamma}\left(K, F_{*} \Omega_{K^{\prime}}^{1}\right)}{\boldsymbol{\Gamma}\left(\mathcal{O}_{K}, F_{*} \Omega_{\mathcal{O}_{K}^{\prime}}^{1}\right)} \rightarrow \frac{\boldsymbol{\Gamma}\left(K, \Omega_{K}^{1}\right)}{\boldsymbol{\Gamma}\left(\mathcal{O}_{K}, \Omega_{\mathcal{O}_{K}}^{1}\right)}\right][-2]
$$

to $\left[\mathbf{G}_{a} \stackrel{1-F}{\rightarrow} \mathbf{G}_{a}\right][-2] \cong \mathbb{Z} / p \mathbb{Z}[-1]$. The morphism

$$
R \boldsymbol{\Gamma}_{x}\left(\mathcal{O}_{K}, U\left(\mu_{p}\right)\right) \rightarrow \mathbb{Z} / p \mathbb{Z}[-1]
$$

thus obtained and the morphism

$$
R \boldsymbol{\Gamma}_{x}\left(\mathcal{O}_{K}, \mu_{p}\right)[1] \rightarrow \mathbb{Z} / p \mathbb{Z}[-1]
$$

coming from $R \boldsymbol{\Gamma}\left(\mathcal{O}_{K}, \mu_{p}\right) \cong \mathbf{U}_{K} /\left(\mathbf{U}_{K}\right)^{p}[-1]$ and $R \boldsymbol{\Gamma}\left(K, \mu_{p}\right) \cong \mathbf{K}^{\times} /\left(\mathbf{K}^{\times}\right)^{p}[-1] \stackrel{v_{K}}{\rightarrow}$ $\mathbb{Z} / p \mathbb{Z}[-1]$ are compatible with the identification (5.2.2.5]).

Proof. By AM76, Lem. 2.1], we have an exact sequence

$$
0 \rightarrow \mathbf{G}_{m} \rightarrow F_{*} \mathbf{G}_{m} \stackrel{\text { dlog }}{\rightarrow} F_{*} \Omega_{K^{\prime}}^{1} \stackrel{C-W^{*}}{\rightarrow} \Omega_{K}^{1} \rightarrow 0
$$

of group schemes over $K$. Since $\mathbf{H}^{1}\left(K, \mathbf{G}_{m}\right)=0$, applying $R \boldsymbol{\Gamma}(K, \cdot)$ gives the exact sequence in the top row of the following diagram:

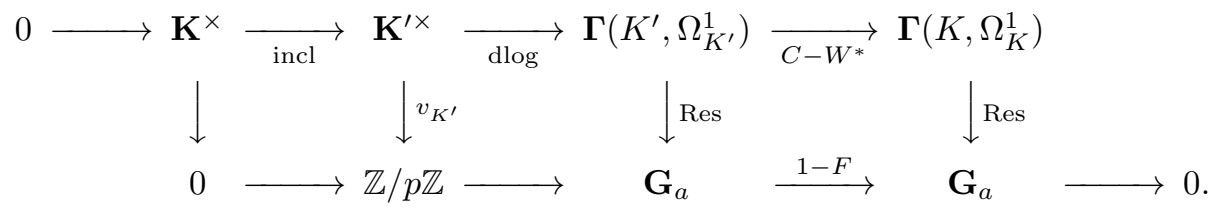

Here $v_{K^{\prime}}$ is the normalized valuation for $K^{\prime}$ and $\boldsymbol{\Gamma}\left(K^{\prime}, \Omega_{K^{\prime}}^{1}\right) \rightarrow \mathbf{G}_{a}$ is the residue map for $K^{\prime}$. The result follows if we check that the squares are commutative. The left two squares are easily seen to be commutative. For the right square, let $T$ be a prime element of $K$ and write

$$
F_{*} \Omega_{K}^{1}=\bigoplus_{i=0}^{p-1} \mathbf{G}_{a} T^{1 / p} d T^{1 / p} / T^{1 / p} \cong \mathbf{G}_{a}^{p}
$$

as group schemes over $K$. The morphism $C-W^{*}$ sends an element $\sum f_{i} T^{i / p} d T^{1 / p} / T^{1 / p}$ to

$$
f_{0} d T / T-\sum\left(f_{i}^{p} T^{i}\right) d T / T
$$

Its residue is $a_{0}-a_{0}^{p}$, where $a_{0}$ is the constant term of $f_{0}$, or the residue of $f_{0}$. This proves the commutativity.

We also call the morphism

$$
R \boldsymbol{\Gamma}_{x}\left(\mathcal{O}_{K}, U\left(\mu_{p}\right)\right) \rightarrow \mathbb{Z} / p \mathbb{Z}[-1] \rightarrow \mathbb{Z}
$$

thus obtained the additive trace morphism. 
Proposition (5.2.2.9). Let $N$ be a finite flat group scheme of height 1 over $\mathcal{O}_{K}$. Then the morphisms

$$
\begin{aligned}
R \boldsymbol{\Gamma}_{x}\left(\mathcal{O}_{K}, V\left(N^{\mathrm{CD}}\right)\right) & \rightarrow R \mathbf{H o m}_{k_{\mathrm{proet}}^{\text {indrat }}}\left(R \boldsymbol{\Gamma}\left(\mathcal{O}_{K}, U(N)\right), R \boldsymbol{\Gamma}_{x}\left(\mathcal{O}_{K}, U\left(\mu_{p}\right)\right)\right) \\
& \rightarrow R \boldsymbol{\Gamma}\left(\mathcal{O}_{K}, U(N)\right)^{\mathrm{SD}}
\end{aligned}
$$

and

$$
\begin{aligned}
R \boldsymbol{\Gamma}\left(\mathcal{O}_{K}, V\left(N^{\mathrm{CD}}\right)\right) & \rightarrow R \mathbf{H o m}_{k_{\text {proet }}^{\text {indrat }}}\left(R \boldsymbol{\Gamma}_{x}\left(\mathcal{O}_{K}, U(N)\right), R \boldsymbol{\Gamma}_{x}\left(\mathcal{O}_{K}, U\left(\mu_{p}\right)\right)\right) \\
& \rightarrow R \boldsymbol{\Gamma}\left(\mathcal{O}_{K}, U(N)\right)^{\mathrm{SD}}
\end{aligned}
$$

in $D\left(k_{\text {proet }}^{\text {indrat }}\right)$ defined by the pairing $V\left(N^{\mathrm{CD}}\right) \times U(N) \rightarrow U\left(\mu_{p}\right)$, the functoriality of $R \tilde{\boldsymbol{\Gamma}}_{x}$ and the additive trace morphism are isomorphisms.

Proof. We only treat the first morphism as the second morphism can be treated similarly. We have a morphism between distinguished triangles

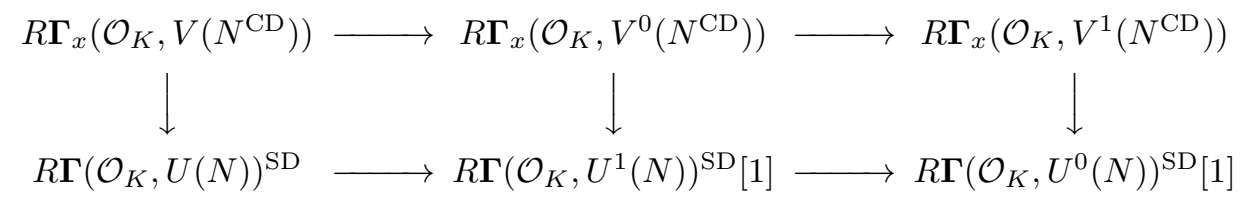

The right two morphisms are isomorphisms by (5.2.2.4). So is the left one.

Proposition (5.2.2.10). Let $N$ be a finite flat group scheme of height 1 over $\mathcal{O}_{K}$. The isomorphisms

$R \boldsymbol{\Gamma}_{x}\left(\mathcal{O}_{K}, V\left(N^{\mathrm{CD}}\right)\right) \rightarrow R \boldsymbol{\Gamma}\left(\mathcal{O}_{K}, U(N)\right)^{\mathrm{SD}}, \quad R \boldsymbol{\Gamma}\left(\mathcal{O}_{K}, V\left(N^{\mathrm{CD}}\right)\right) \rightarrow R \boldsymbol{\Gamma}\left(\mathcal{O}_{K}, U(N)\right)^{\mathrm{SD}}$ in the previous proposition and the morphisms

$$
R \boldsymbol{\Gamma}_{x}\left(\mathcal{O}_{K}, N^{\mathrm{CD}}\right) \rightarrow R \boldsymbol{\Gamma}\left(\mathcal{O}_{K}, N\right)^{\mathrm{SD}}[-1], \quad R \boldsymbol{\Gamma}\left(\mathcal{O}_{K}, N^{\mathrm{CD}}\right) \rightarrow R \boldsymbol{\Gamma}\left(\mathcal{O}_{K}, N\right)^{\mathrm{SD}}[-1]
$$

given in (5.2.1.2) are compatible under the identifications (5.2.2.5) and (5.2.2.6).

Proof. Applying the functoriality of $R \tilde{\boldsymbol{\Gamma}}_{x}$ to the diagram (5.2.2.7), we have commutative diagrams

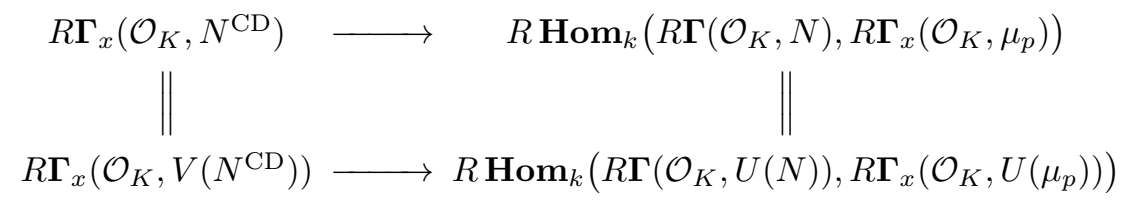

and

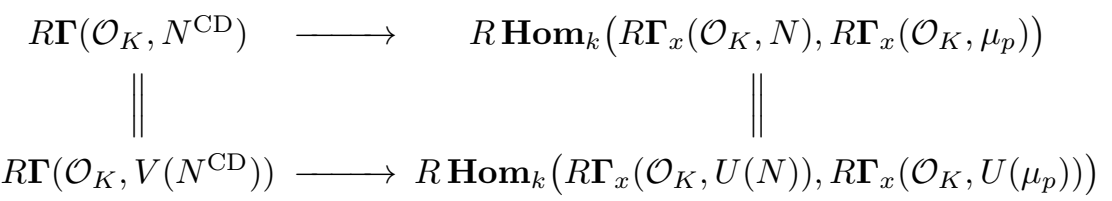

in $D\left(k_{\text {proet }}^{\text {indrat }}\right)$. The morphism in (5.2.1.2) is given by applying the morphism

$$
R \boldsymbol{\Gamma}_{x}\left(\mathcal{O}_{K}, \mu_{p}\right)[1] \rightarrow \mathbb{Z} / p \mathbb{Z}[-1] .
$$

The morphism in (5.2.2.9) is given by applying the morphism

$$
R \boldsymbol{\Gamma}_{x}\left(\mathcal{O}_{K}, U\left(\mu_{p}\right)\right) \rightarrow \mathbb{Z} / p \mathbb{Z}[-1] .
$$

As they are compatible by (5.2.2.8), the result follows. 
This finishes the proof of (5.2.1.2) and (5.2.2.1).

\subsubsection{Bertapelle's isomorphism.}

Proposition (5.2.3.1). Assume that $K$ has equal characteristic and $k$ is algebraically closed. Let $A$ be a semistable abelian variety over $K$. Suppose that we take the morphism constructed in (5.2.1.4) as the definition of Bester's isomorphism that Bertapelle used in [Ber03. Thm. 1]. Then the resulting isomorphism

$$
\psi_{A}: H^{1}\left(K, A^{\vee}\right) \stackrel{\sim}{\rightarrow} \operatorname{Ext}_{k_{\text {proet }}^{\text {indrat }}}^{1}(\boldsymbol{\Gamma}(A), \mathbb{Q} / \mathbb{Z})
$$

Bertapelle constructed in [Ber03, Thm. 2] coincides with our morphism $\theta_{A}^{+1}(k)$.

We prove this below. For the moment, $k$ is assumed to be a general perfect field. First we need a preparation about the finite flat site of $\mathcal{O}_{K}$.

Proposition (5.2.3.2). Let $k^{\prime} \in k^{\text {indrat }}$. The local ring (for the Zariski topology) of $\mathbf{O}_{K}\left(k^{\prime}\right)$ at any maximal ideal is Henselian.

Proof. By (3.1.1) (a) a maximal ideal $\mathfrak{n}$ of $\mathbf{O}_{K}\left(k^{\prime}\right)$ is of the form $\mathbf{p}_{K}\left(k^{\prime}\right)+\mathbf{O}_{K}(\mathfrak{m})$ for some $\mathfrak{m} \in \operatorname{Spec} k^{\prime}$, with residue field $k^{\prime} / \mathfrak{m}$. By (3.1.1) (b), the local $\operatorname{ring} \mathbf{O}_{K}\left(k^{\prime}\right)_{\mathfrak{n}}$ is given by the filtered direct limit of $\mathbf{O}_{K}\left(k^{\prime}[1 / e]\right)=\mathbf{O}_{K}\left(k^{\prime} /(1-e)\right)$ for the idempotents $e \in k^{\prime} \backslash \mathfrak{m}$.

Let $f_{1}, \ldots, f_{n} \in \mathbf{O}_{K}\left(k^{\prime}\right)\left[x_{1}, \ldots, x_{n}\right]$ and $\alpha=\left(\alpha_{1}, \ldots, \alpha_{n}\right) \in \mathbf{O}_{K}\left(k^{\prime}\right)^{n}$. Suppose that the images $\bar{f}_{i}(\alpha) \in k^{\prime} / \mathfrak{m}$ are zero for all $i$ and $\operatorname{det}\left(\partial \bar{f}_{i} / \partial x_{j}\right)(\alpha) \neq 0$. We need to show that the polynomial system $\left(f_{1}, \ldots, f_{n}\right)$ has a root in $\mathbf{O}_{K}\left(k^{\prime}\right)_{\mathfrak{n}}$ whose reduction is $\bar{\alpha} \in k^{\prime} / \mathfrak{m}$. It is enough to show the existence of a root in $\mathbf{O}_{K}\left(k^{\prime}\right)$ after replacing $k^{\prime}$ by $k^{\prime}[1 / e]$ for an idempotent $e \in k^{\prime} \backslash \mathfrak{m}$. Since the element $\operatorname{det}\left(\partial f_{i} / \partial x_{j}\right)(\alpha) \in \mathbf{O}_{K}\left(k^{\prime}\right)$ has non-zero image in $k^{\prime} / \mathfrak{m}$, (3.1.1) (ㄷ) shows that $\operatorname{det}\left(\partial f_{i} / \partial x_{j}\right)(\alpha) \in \mathbf{U}_{K}\left(k^{\prime}\right)$ after replacing $k^{\prime}$. Since $\mathbf{O}_{K}\left(k^{\prime}\right)$ is $\mathbf{p}_{K}\left(k^{\prime}\right)$-adically complete, Hensel's lemma shows the existence of a desired root.

Recall that a morphism of schemes is finite locally free if and only if it is finite flat locally of finite presentation (Bou98, Chap. II, §5, No. 2, Cor. 2 to Thm. 1], Gro64, Prop. 1.4.7]). For a commutative $\operatorname{ring} S$, we denote by Spec $S_{\mathrm{ffl}}$ the finite flat site of $S$, namely the category of finite locally free $S$-algebras where a covering of an object $S^{\prime}$ is a finite family $\left\{S_{i}^{\prime}\right\}$ of finite locally free $S^{\prime}$-algebras such that $\prod S_{i}^{\prime}$ is faithfully flat over $S^{\prime}$.

Proposition (5.2.3.3). Let $k^{\prime} \in k^{\text {indrat }}$ and $S$ a finite $\mathbf{O}_{K}\left(k^{\prime}\right)$-algebra. Then any fppf covering of Spec $S$ can be refined by a finite locally free covering. That is, for any faithfully flat $S$-algebra $S^{\prime}$ of finite presentation, there exist a faithfully flat finite locally free $S$-algebra $S^{\prime \prime}$ and an $S$-algebra homomorphism $S^{\prime} \rightarrow S^{\prime \prime}$. In particular, the continuous map $g:$ Spec $S_{\mathrm{fppf}} \rightarrow$ Spec $S_{\mathrm{ffl}}$ of sites defined by the identity induces an exact pushforward functor $g_{*}: \operatorname{Set}\left(S_{\mathrm{fppf}}\right) \rightarrow \operatorname{Set}\left(S_{\mathrm{ffl}}\right)$.

Proof. Refine Spec $S^{\prime}$ by a quasi-finite flat covering Spec $S^{\prime \prime \prime}$ of finite presentation (Gro67, 17.16.2]). Since $S$ is finite, the previous proposition shows that the local ring of $S$ at any maximal ideal of $S$ is Henselian. Hence Spec $S^{\prime \prime \prime}$ can be refined by a finite locally free covering.

The following is stated in Suz13, Rmk. 2.7.6 (3)]. 
Proposition (5.2.3.4). Let $j: \operatorname{Spec} K_{\mathrm{fppf}} / k^{\text {indrat }} \hookrightarrow \operatorname{Spec} O_{K, \mathrm{fppf}} / k^{\text {indrat }}$ be the morphism induced by the open immersion $\operatorname{Spec} K \hookrightarrow \operatorname{Spec} \mathcal{O}_{K}$. Denote by $j_{\text {! }}$ the zero extension functor by $j$ (AGV72, Exp. III, 5.3, 3)]). Then $R \boldsymbol{\Gamma}\left(\mathcal{O}_{K}, j_{!} A\right)=0$ for any $A \in \operatorname{Ab}\left(K_{\text {fppf }} / k_{\text {et }}^{\text {indrat }}\right)$.

Proof. It is enough to show that $R \Gamma\left(\mathbf{O}_{K}\left(k^{\prime}\right), j ! A\right)=0$ for any $k^{\prime} \in k^{\text {indrat }}$. Let $g$ : Spec $\mathbf{O}_{K}\left(k^{\prime}\right)_{\mathrm{fppf}} \rightarrow \operatorname{Spec} \mathbf{O}_{K}\left(k^{\prime}\right)_{\mathrm{ffl}}$ be the continuous map defined by the identity. We have $R \Gamma\left(\mathbf{O}_{K}\left(k^{\prime}\right), j_{!} A\right)=R \Gamma\left(\mathbf{O}_{K}\left(k^{\prime}\right)_{\mathrm{ff}}, g_{*} j_{!} A\right)$ by the previous proposition. The $j$ here should be understood as the open immersion $\operatorname{Spec} \mathbf{K}\left(k^{\prime}\right) \hookrightarrow \operatorname{Spec} \mathbf{O}_{K}\left(k^{\prime}\right)$. Let $S$ be a non-zero finite locally free $\mathbf{O}_{K}\left(k^{\prime}\right)$-algebra of (locally constant) rank $r \geq 1$. Then the norm $N_{S / \mathbf{O}_{K}\left(k^{\prime}\right)} \pi=\pi^{r}$ does not belong to $\mathbf{U}_{K}\left(k^{\prime}\right)$. Hence the homomorphism $\mathbf{O}_{K}\left(k^{\prime}\right) \rightarrow S$ cannot factor through $\mathbf{K}\left(k^{\prime}\right)$. Therefore $g_{*} j_{!}=0$ by the construction of the zero-extension functor $j$ !

Bertapelle extended Bester's group $\mathscr{F}(N)$ for quasi-finite flat separated group schemes $N$ over $\mathcal{O}_{K}$. For such a group $N$, we denote its finite part by $N^{f}$. By [Ber03, 33.1 Example], we have $\mathscr{F}(N)=\mathscr{F}\left(N^{f}\right)$.

Proposition (5.2.3.5). Let $N$ be a quasi-finite flat separated group scheme over $\mathcal{O}_{K}$ with finite part $N^{f}$. Then we have $R \boldsymbol{\Gamma}\left(\mathcal{O}_{K}, N\right)=R \boldsymbol{\Gamma}\left(\mathcal{O}_{K}, N^{f}\right) \in D(\mathrm{PAlg} / k)$ and it is $P$-acyclic. In particular, there exists a canonical isomorphism $L \pi_{0} R \boldsymbol{\Gamma}\left(\mathcal{O}_{K}, N\right) \cong$ $\mathscr{F}(N)$ compatible with the isomorphism $L \pi_{0} R \boldsymbol{\Gamma}\left(\mathcal{O}_{K}, N^{f}\right) \cong \mathscr{F}\left(N^{f}\right)$ of (5.2.1.3).

Proof. Let $Y=N \backslash N^{f}$ be the part of $N$ finite over $K$. Let $k^{\prime} \in k^{\text {indrat }}$ and $S$ a finite locally free $\mathbf{O}_{K}\left(k^{\prime}\right)$-algebra. If there is an $\mathbf{O}_{K}\left(k^{\prime}\right)$-scheme morphism $\operatorname{Spec} S \rightarrow Y$, then $S$ has to be a $\mathbf{K}\left(k^{\prime}\right)$-algebra, which happens only when $S=0$ by the same reasoning as the proof of the previous proposition. Hence $\Gamma\left(S, N^{f}\right)=\Gamma(S, N)$. Therefore $N^{f}=N$ as sheaves on $\operatorname{Spec} \mathbf{O}_{K}\left(k^{\prime}\right)_{\mathrm{fff}}$. Hence (5.2.3.3) implies that $R \Gamma\left(\mathbf{O}_{K}\left(k^{\prime}\right), N^{f}\right)=R \Gamma\left(\mathbf{O}_{K}\left(k^{\prime}\right), N\right)$, and we have $R \boldsymbol{\Gamma}\left(\mathcal{O}_{K}, N^{f}\right)=R \boldsymbol{\Gamma}\left(\mathcal{O}_{K}, N\right)$.

Now we recall the definition of Bertapelle's isomorphism in the case the abelian variety $A$ has semistable reduction. Assume that $k$ is algebraically closed. Let $\mathcal{A}$ be the Néron model of $A$ and $\mathcal{A}_{0}$ the maximal open subgroup scheme with connected special fiber. Let $n \geq 1$ be an integer that kills $\pi_{0}\left(\mathcal{A}_{x}\right)$. Let $\mathcal{A}[n]^{f}$ be the finite part of the $n$-torsion part $\mathcal{A}[n]$ and $\left(\mathcal{A}[n]^{f}\right)_{K}=\mathcal{A}[n]^{f} \times_{\mathcal{O}_{K}} K$. The inclusion $\left(\mathcal{A}[n]^{f}\right)_{K} \hookrightarrow A[n]$ defines a surjection $A[n]^{\mathrm{CD}} \rightarrow\left(\mathcal{A}[n]^{f}\right)_{K}^{\mathrm{CD}}$. By [Ber03, Lem. 14], this canonically extends to a morphism

$$
\mathcal{A}^{\vee}[n] \rightarrow\left(\mathcal{A}[n]^{f}\right)^{\mathrm{CD}}
$$

of group schemes over $\mathcal{O}_{K}$, which induces an isomorphism

$$
H_{x}^{2}\left(\mathcal{O}_{K}, \mathcal{A}^{\vee}[n]\right) \stackrel{\sim}{\rightarrow} H_{x}^{2}\left(\mathcal{O}_{K},\left(\mathcal{A}[n]^{f}\right)^{\mathrm{CD}}\right) .
$$

We denote by $\theta_{\mathcal{A}[n]^{f}}^{2}$ the isomorphism

$$
H_{x}^{2}\left(\mathcal{O}_{K},\left(\mathcal{A}[n]^{f}\right)^{\mathrm{CD}}\right) \stackrel{\sim}{\rightarrow} \mathscr{F}\left(\mathcal{A}[n]^{f}\right)^{\mathrm{PD}}
$$

in (5.2.1.4) for $N=\mathcal{A}[n]^{f}$. Combining the above two isomorphisms and $\mathscr{F}\left(\mathcal{A}[n]^{f}\right) \cong$ $\mathscr{F}(\mathcal{A}[n])$, we have an isomorphism

$$
H_{x}^{2}\left(\mathcal{O}_{K}, \mathcal{A}^{\vee}[n]\right) \stackrel{\sim}{\rightarrow} \mathscr{F}(\mathcal{A}[n])^{\mathrm{PD}} .
$$

We denote this isomorphism by $\psi_{\mathcal{A}[n]}$. Note that

$$
H_{x}^{2}\left(\mathcal{O}_{K}, \mathcal{A}_{0}^{\vee}\right)=H_{x}^{2}\left(\mathcal{O}_{K}, \mathcal{A}^{\vee}\right)=H^{1}\left(K, A^{\vee}\right) .
$$


Hence the exact sequence $0 \rightarrow \mathcal{A}^{\vee}[n] \rightarrow \mathcal{A}^{\vee} \stackrel{n}{\rightarrow} \mathcal{A}_{0}^{\vee} \rightarrow 0$ induces a surjection

$$
H_{x}^{2}\left(\mathcal{O}_{K}, \mathcal{A}^{\vee}[n]\right) \rightarrow H^{1}\left(K, A^{\vee}\right)[n] .
$$

By [Ber03, $§ 3.1$, Examples], the map $\mathscr{F}\left(\mathcal{A}_{0}[n]\right) \rightarrow \mathscr{F}(\mathcal{A}[n])$ is injective, and we have

$$
\begin{aligned}
\mathscr{F}\left(\mathcal{A}_{0}[n]\right) & =\operatorname{Coker}\left(\pi_{1} \boldsymbol{\Gamma}\left(\mathcal{O}_{K}, \mathcal{A}_{0}\right) \stackrel{n}{\rightarrow} \pi_{1} \boldsymbol{\Gamma}\left(\mathcal{O}_{K}, \mathcal{A}_{0}\right)\right) \\
& =\operatorname{Coker}\left(\pi_{1} \boldsymbol{\Gamma}\left(\mathcal{O}_{K}, \mathcal{A}\right) \stackrel{n}{\rightarrow} \pi_{1} \boldsymbol{\Gamma}\left(\mathcal{O}_{K}, \mathcal{A}\right)\right) \\
& \left.\left.=\operatorname{Coker}\left(\pi_{1} \boldsymbol{\Gamma}(K, A)\right) \stackrel{n}{\rightarrow} \pi_{1} \boldsymbol{\Gamma}(K, A)\right)\right)
\end{aligned}
$$

By the proof of $\left[\right.$ Ber03, Thm. 2], the isomorphism $\psi_{\mathcal{A}[n]}: H_{x}^{2}\left(\mathcal{O}_{K}, \mathcal{A}^{\vee}[n]\right) \stackrel{\sim}{\rightarrow} \mathscr{F}(\mathcal{A}[n])^{\mathrm{PD}}$ induces an isomorphism

$$
H^{1}\left(K, A^{\vee}\right)[n] \stackrel{\sim}{\rightarrow}\left(\pi_{1} \boldsymbol{\Gamma}(K, A)\right)^{\mathrm{PD}}[n]
$$

on the quotients. The resulting isomorphism

$$
H^{1}\left(K, A^{\vee}\right) \stackrel{\sim}{\rightarrow}\left(\pi_{1} \Gamma(K, A)\right)^{\mathrm{PD}}
$$

is the definition of Bertapelle's isomorphism in the semistable case. We denote it by $\psi_{A}$.

Hence, to prove (5.2.3.1), it is enough to show the following.

Proposition (5.2.3.6). Let $A$ and $n$ be as above. There exists a diagram (commutativity to be mentioned below)

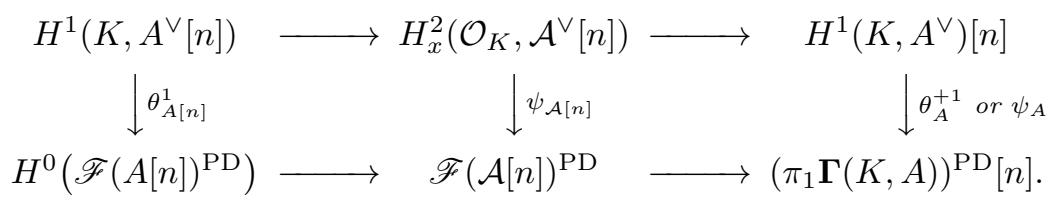

Here we define

$$
\mathscr{F}(A[n])^{\mathrm{PD}}=R \operatorname{Hom}_{k}(R \Gamma(K, A[n]), \mathbb{Q} / \mathbb{Z}) .
$$

The left vertical morphism $\theta_{A[n]}^{1}$ is the isomorphism induced on $H^{1}$ by the isomorphism given in (5.2.2.1). We denoted $\theta_{A}^{+1}=\theta_{A}^{+1}(k)$. The horizontal homomorphisms in the left square are to be constructed below, and those in the right square are already mentioned. The upper horizontal homomorphisms are surjective.

The left square is commutative. If we use Bertapelle's isomorphism $\psi_{A}$ for the right vertical arrow, then the right square is commutative (by definition). If we use our morphism $\theta_{A}^{+1}$, then the total square (omitting the middle $\psi_{\mathcal{A}[n]}$ ) is commutative.

As a consequence, we have $\theta_{A}^{+1}=\psi_{A}$ and the right square is commutative.

Proof. The upper horizontal homomorphism in the left square is defined as the coboundary map of the localization sequence for $\mathcal{A}^{\vee}[n]$. It is surjective since

$$
R \Gamma\left(\mathcal{O}_{K}, \mathcal{A}^{\vee}[n]\right)=R \Gamma\left(\mathcal{O}_{K}, \mathcal{A}^{\vee}[n]^{f}\right)
$$

by (5.2.3.5) and this is concentrated in degrees 0 and 1 . The composite of the upper two horizontal homomorphisms is induced by the inclusion $A^{\vee}[n] \hookrightarrow A^{\vee}$. By 
(5.2.3.5) and by the same calculation as the second paragraph after (5.2.1.2), we have

$$
\begin{aligned}
\mathscr{F}(\mathcal{A}[n])^{\mathrm{PD}} & =R \operatorname{Hom}_{\mathrm{PFEt} / k}\left(L \pi_{0} R \boldsymbol{\Gamma}\left(\mathcal{O}_{K}, \mathcal{A}[n]\right), \mathbb{Q} / \mathbb{Z}\right) \\
& =R \operatorname{Hom}_{k}\left(R \boldsymbol{\Gamma}\left(\mathcal{O}_{K}, \mathcal{A}[n]\right), \mathbb{Q} / \mathbb{Z}\right) .
\end{aligned}
$$

The lower horizontal homomorphism in the left square is defined by dualizing $R \boldsymbol{\Gamma}\left(\mathcal{O}_{K}, \mathcal{A}[n]\right) \rightarrow R \boldsymbol{\Gamma}(K, A[n])$. The left square is decomposed into

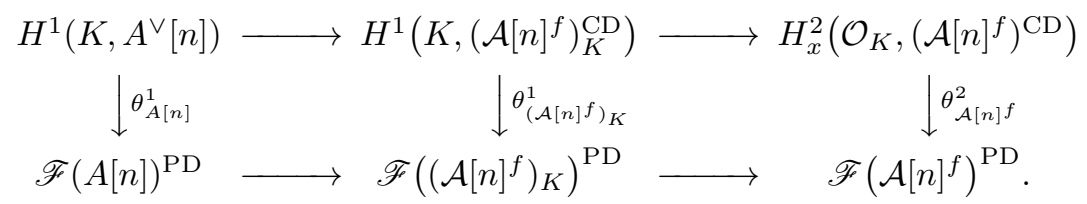

The commutativity of the left square is the functoriality of the isomorphisms $\theta_{N}^{1}$ for finite flat group schemes $N$ over $K$, which is easy to check. That of the right square follows from (5.2.2.2).

We show the commutativity of the total square with $\theta_{A}^{+1}$ used for the right vertical arrow. With the identification $\mathscr{F}\left(\mathcal{A}_{0}[n]\right)^{\mathrm{PD}}=\pi_{1} \boldsymbol{\Gamma}(K, A)^{\mathrm{PD}}[n]$ used earlier, the lower two horizontal homomorphisms can be written as the $H^{0}$ of the Pontryagin dual of the morphisms

$$
L \pi_{0} R \boldsymbol{\Gamma}\left(\mathcal{O}_{K}, \mathcal{A}_{0}[n]\right) \rightarrow L \pi_{0} R \boldsymbol{\Gamma}\left(\mathcal{O}_{K}, \mathcal{A}[n]\right) \rightarrow L \pi_{0} R \boldsymbol{\Gamma}(K, A[n]) .
$$

The $H^{0}$ of the composite of these morphisms is

$$
\operatorname{Coker}\left(\pi_{1} \boldsymbol{\Gamma}(K, A) \stackrel{n}{\rightarrow} \pi_{1} \boldsymbol{\Gamma}(K, A)\right) \rightarrow H^{0} L \pi_{0} R \boldsymbol{\Gamma}(K, A[n]) .
$$

This comes from the $H^{0}$ of the distinguished triangle

$$
L \pi_{0} R \boldsymbol{\Gamma}(K, A)[-1] \stackrel{n}{\rightarrow} L \pi_{0} R \boldsymbol{\Gamma}(K, A)[-1] \rightarrow L \pi_{0} R \boldsymbol{\Gamma}(K, A[n]) .
$$

Therefore it is enough to construct a morphism of distinguished triangles

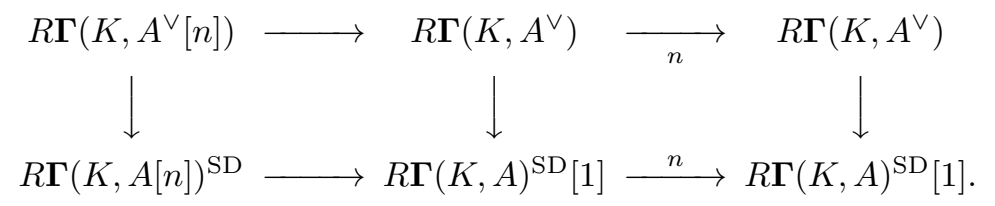

(The left square gives the total square in the statement.) For this, note that we have a morphism of triangles from the short exact sequence $0 \rightarrow A^{\vee}[n] \rightarrow A^{\vee} \rightarrow A^{\vee} \rightarrow 0$ to

$$
R \operatorname{Hom}_{K}\left(A[n], \mathbf{G}_{m}\right) \rightarrow R \operatorname{Hom}_{K}\left(A, \mathbf{G}_{m}\right)[1] \rightarrow R \operatorname{Hom}_{K}\left(A, \mathbf{G}_{m}\right)[1] .
$$

Applying the morphism of functoriality of $R \boldsymbol{\Gamma}$ to it and using the trace morphism $R \boldsymbol{\Gamma}\left(K, \mathbf{G}_{m}\right) \rightarrow \mathbb{Z}$, we get the desired morphism of the distinguished triangles.

This completes the proof of (5.2.3.1) and hence (5.2.1).

\subsection{Bégueri's isomorphism.}

Proposition (5.3.1). Assume that $k$ is algebraically closed. The morphism

$$
\theta_{A}^{+1}(k): H^{1}\left(K, A^{\vee}\right) \rightarrow \operatorname{Ext}_{k_{\text {proet }}^{\text {indrat }}}^{1}(\boldsymbol{\Gamma}(A), \mathbb{Q} / \mathbb{Z})
$$

of (4.2.1) coincides with Bégueri's isomorphism [Bég81, Thm. 8.3.6] when K has mixed characteristic. 
Proof. Let $n \geq 1$. We have morphisms

$$
\begin{aligned}
R \operatorname{Hom}_{K}\left(\mathbb{Z} / n \mathbb{Z}, A^{\vee}\right) & \rightarrow R \operatorname{Hom}_{K}\left(\mathbb{Z} / n \mathbb{Z}, R \operatorname{Hom}_{K}\left(A, \mathbf{G}_{m}\right)\right)[1] \\
& \rightarrow R \operatorname{Hom}_{K}\left(A, R \operatorname{Hom}_{K}\left(\mathbb{Z} / n \mathbb{Z}, \mathbf{G}_{m}\right)\right)[1] \\
& =R \operatorname{Hom}_{K}\left(A, \mu_{n}\right)[1] \\
& \rightarrow R \operatorname{Hom}_{k_{\text {proet }}^{\text {indrat }}}\left(R \boldsymbol{\Gamma}(A), R \boldsymbol{\Gamma}\left(\mu_{n}\right)\right)[1] \\
& \rightarrow R \operatorname{Hom}_{k_{\text {proet }}^{\text {indrat }}}(R \boldsymbol{\Gamma}(A), \mathbb{Z} / n \mathbb{Z})
\end{aligned}
$$

in $D(\mathrm{Ab})$, where the last morphism comes from the Kummer sequence and the trace morphism $R \boldsymbol{\Gamma}\left(\mathbf{G}_{m}\right)=\mathbf{K}^{\times} \rightarrow \mathbb{Z}$. Using the sequence $0 \rightarrow \mathbb{Z} \rightarrow \mathbb{Z} \rightarrow \mathbb{Z} / n \mathbb{Z} \rightarrow 0$, this and our duality morphism fit in the following commutative diagram:

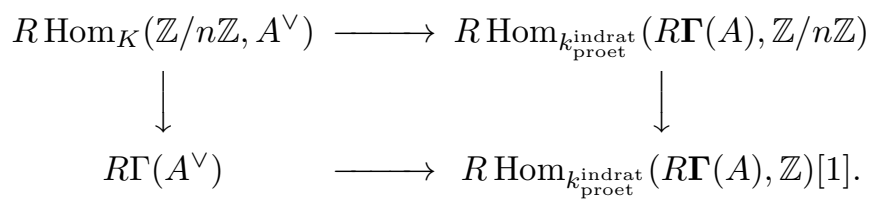

By construction, the top horizontal morphism in degree 1 is given as follows. First, for $B, C \in \mathrm{Ab}\left(K_{\mathrm{fppf}} / k_{\mathrm{et}}^{\text {indrat }}\right)$, the sheaf $\operatorname{Hom}_{\mathbf{K}}(B, C):=\tilde{\boldsymbol{\Gamma}} \operatorname{Hom}_{K}(B, C)$ on Spec $k_{\text {proet }}^{\text {indrat }}$ is the pro-étale sheafification of the étale sheaf $k^{\prime} \mapsto \operatorname{Hom}_{\left(\mathbf{K}\left(k^{\prime}\right), k^{\prime}\right)}(B, C)$, where $\operatorname{Hom}_{\left(\mathbf{K}\left(k^{\prime}\right), k^{\prime}\right)}$ is the Hom functor for the localization of Spec $K_{\mathrm{fppf}} / k_{\mathrm{et}}^{\text {indrat }}$ at the object $\left(\mathbf{K}\left(k^{\prime}\right), k^{\prime}\right)$. For any $n$, the $n$-th cohomology $\operatorname{Ext}_{\mathbf{K}}^{n}(B, C)$ of the derived functor $R \operatorname{Hom}_{\mathbf{K}}(B, C)=R \tilde{\boldsymbol{\Gamma}} R \mathbf{H o m}_{K}(B, C)$ is the pro-étale sheafification of the presheaf $k^{\prime} \mapsto \operatorname{Ext}_{\left(\mathbf{K}\left(k^{\prime}\right), k^{\prime}\right)}^{n}(B, C)$. Now let $0 \rightarrow A^{\vee} \rightarrow X \rightarrow \mathbb{Z} / n \mathbb{Z} \rightarrow 0$ be an extension over $K$. The long exact sequence for $R$ Hom $_{\mathbf{K}}$ gives

$$
0 \rightarrow \operatorname{Ext}_{\mathbf{K}}^{1}\left(\mathbb{Z} / n \mathbb{Z}, \mathbf{G}_{m}\right) \rightarrow \operatorname{Ext}_{\mathbf{K}}^{1}\left(X, \mathbf{G}_{m}\right) \rightarrow \operatorname{Ext}_{\mathbf{K}}^{1}\left(A^{\vee}, \mathbf{G}_{m}\right) \rightarrow 0,
$$

or

$$
0 \rightarrow \mathbf{H}^{1}\left(\mu_{n}\right) \rightarrow \operatorname{Ext}_{\mathbf{K}}^{1}\left(X, \mathbf{G}_{m}\right) \rightarrow \boldsymbol{\Gamma}(A) \rightarrow 0,
$$

hence an element of $\operatorname{Ext}_{k_{\text {proet }}^{\text {indrat }}}^{1}\left(\boldsymbol{\Gamma}(A), \mathbf{H}^{1}\left(\mu_{n}\right)\right)$, hence an element of $\operatorname{Ext}_{k_{\text {proet }}^{\text {ind }}}^{1}(\boldsymbol{\Gamma}(A), \mathbb{Z} / n \mathbb{Z})$.

This homomorphism fits in the following commutative diagram:

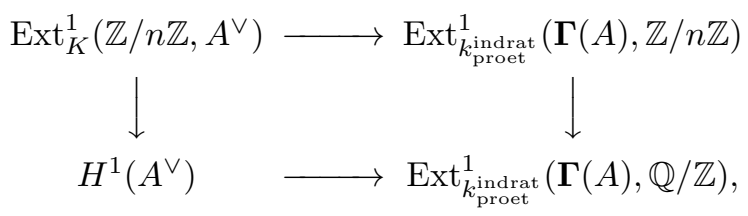

where the bottom arrow is our homomorphism $\theta_{A}^{+1}(k)$. The description above shows that the top arrow is the same as Bégueri's homomorphism (Bég81, Lem. 8.2.2, Thm. 8.3.6]). This completes the comparison.

\section{Galois Descent}

Proposition (6.1). Let $A$ be an abelian variety over $K$ and $L / K$ a finite Galois extension. If (4.1.2) is for $A$ over $L$, then so is for $A$ over $K$.

We prove this below. We need notation and lemmas on group (co)homology. The basic reference is [Ser79, VII, VIII, IX]. We need Tate cohomology in the setting of derived categories of sheaves of $G$-modules on sites. For the purpose of this section, available literature seems to be either less general or more topological (in the sense 
of equivariant stable homotopy theory) than what is needed here. Hence we include some basics for the convenience of the reader.

Let $G$ be a finite (abstract) group and $S$ a site. We denote the category of (left) $G$-modules (or $\mathbb{Z}[G]$-modules) by $G$-Mod, so that $G$-Mod $(S)$ is the (abelian) category of sheaves of $G$-modules on $S$. For a complex $M \in D(G-\operatorname{Mod}(S))$, we define its group cohomology, group homology by

$$
R \Gamma(G, M)=R \operatorname{Hom}_{G-\operatorname{Mod}(S)}(\mathbb{Z}, M), \quad L \Delta(G, M)=\mathbb{Z} \otimes_{\mathbb{Z}[G]}^{L} M,
$$

respectively 15 They are objects of $D(S)=D(\mathrm{Ab}(S))$. Let $C(G)$ be the standard resolution

$$
\cdots \rightarrow \mathbb{Z}\left[G^{3}\right] \rightarrow \mathbb{Z}\left[G^{2}\right] \rightarrow \mathbb{Z}[G]
$$

of the trivial $G$-module $\mathbb{Z}$ ([Ser79, VII, §3]). This is viewed as a complex $\left\{C(G)^{i}\right\}_{i \leq 0}$ concentrated in non-positive degrees (in cohomological grading, as for all the complexes in this paper). Let $C^{\vee}(G)=\operatorname{Hom}_{\mathrm{Ab}}(C(G), \mathbb{Z})$. Define the standard complete resolution $\hat{C}(G)$ of $\mathbb{Z}$ [Mil06, I, $\S 0$, "Tate (modified) cohomology groups"] to be the complex

$$
\cdots \rightarrow \mathbb{Z}\left[G^{2}\right] \rightarrow \mathbb{Z}[G] \stackrel{N}{\rightarrow} \mathbb{Z}[G] \rightarrow \mathbb{Z}\left[G^{2}\right] \rightarrow \cdots,
$$

where the map $N$ from the degree 0 term to the degree 1 term is the norm map $\sum_{\sigma \in G} \sigma$, and the non-positive degree part of the complex is $C(G)$ and the positive degree part $C^{\vee}(G)[-1]$. The morphism of complexes $C(G) \rightarrow C^{\vee}(G)$ induced by $N: \mathbb{Z}[G] \rightarrow \mathbb{Z}[G]$ in degree zero is also denoted by $N$. Then $\hat{C}(G)$ is the mapping fiber of $N: C(G) \rightarrow C^{\vee}(G)$. For a bounded complex $M$ of sheaves of $G$-modules on $S$, we define the Tate cohomology as the sheaf-Hom (total) complex

$$
R \hat{\Gamma}(G, M)=\operatorname{Hom}_{G-\operatorname{Mod}(S)}(\hat{C}(G), M)
$$

viewed as an object of $D(S)$. Note that $R \mathbf{H o m}_{G-\operatorname{Mod}(S)}(\hat{C}(G), M)=0$ since $\hat{C}(G)$ is an exact complex.

We relate the three functors $R \Gamma, L \Delta$ and $R \hat{\Gamma}$ and show that $R \hat{\Gamma}$ factors through the derived category. We have $R \Gamma(G, M)=R \mathbf{H o m}_{G-\operatorname{Mod}(S)}(C(G), M)$. Consider the hyperext spectral sequence

$$
E_{1}^{i j}=\prod_{i^{\prime}+i^{\prime \prime}=i} \mathbf{E x t}_{G-\operatorname{Mod}(S)}^{j}\left(C(G)^{-i^{\prime}}, M^{i^{\prime \prime}}\right) \Longrightarrow H^{i+j} R \mathbf{H o m}_{G-\operatorname{Mod}(S)}(C(G), M),
$$

constructed in the same way as [ML63, Thm. 12.2]. This is convergent since $C(G)$ is bounded above and $M$ bounded below. Since each term of $C(G)$ is finite free over $\mathbb{Z}[G]$, we have $E_{1}^{i j}=0$ for any $i, j$ with $j \geq 1$. Hence $R \operatorname{Hom}_{G-\operatorname{Mod}(S)}(C(G), M)$ is represented by the total complex $\operatorname{Hom}_{G-\operatorname{Mod}(S)}(C(G), M)$. Thus

$$
R \Gamma(G, M)=\operatorname{Hom}_{G-\operatorname{Mod}(S)}(C(G), M) .
$$

\footnotetext{
${ }^{15}$ Using $\Delta$, the Greek letter next to $\Gamma$, to denote homology is non-standard. There seems to be no widely used notation for homology in derived categories that is parallel to cohomology $R \Gamma$. Perhaps $\Lambda$ instead, in accordance with the definition of the homology $X \mapsto E \wedge X$ of a spectrum $E ?$
} 
Similarly we have $L \Delta(G, M)=C(G) \otimes_{\mathbb{Z}[G]} M$. The $n$-th term of $C(G) \otimes_{\mathbb{Z}[G]} M$ for each $n$ is

$$
\begin{aligned}
\bigoplus_{i+j=n} C(G)^{i} \otimes_{\mathbb{Z}[G]} M^{j} & =\bigoplus_{i+j=n} \operatorname{Hom}_{G-\operatorname{Mod}(S)}\left(C^{\vee}(G)^{-i}, M^{j}\right) \\
& =\prod_{i+j=n} \operatorname{Hom}_{G-\operatorname{Mod}(S)}\left(C^{\vee}(G)^{-i}, M^{j}\right),
\end{aligned}
$$

where the second equality comes from the property that $C^{\vee}(G)$ is bounded below and $M$ bounded above. The last term is the $n$-th term of $\mathbf{H o m}_{G-\operatorname{Mod}(S)}\left(C^{\vee}(G), M\right)$. Thus

$$
L \Delta(G, M)=\operatorname{Hom}_{G-\operatorname{Mod}(S)}\left(C^{\vee}(G), M\right) .
$$

In particular, the norm map $N: C(G) \rightarrow C^{\vee}(G)$ induces a morphism $L \Delta(G, M) \rightarrow$ $R \Gamma(G, M)$ in $D(S)$, which we denote by the same symbol $N$. Combining (6.2), (6.3), (6.4) and the mapping fiber distinguished triangle

$$
\hat{C}(G) \rightarrow C(G) \stackrel{N}{\rightarrow} C^{\vee}(G),
$$

we have a distinguished triangle

$$
L \Delta(G, M) \stackrel{N}{\rightarrow} R \Gamma(G, M) \rightarrow R \hat{\Gamma}(G, M) .
$$

In particular, if $M$ is an exact complex, then $L \Delta(G, M), R \Gamma(G, M)$ and hence $R \hat{\Gamma}(G, M)$ are all zero in $D(S)$. Therefore the assignment $M \mapsto R \hat{\Gamma}(G, M)$ defines a well-defined triangulated functor $D^{b}(G-\operatorname{Mod}(S)) \rightarrow D(S)$ by $\underline{\text { KS06}}$, Prop. 10.3.3] 16

We need to know how dual of group cohomology and group cohomology of dual are related. Let $M \in D^{b}(G-\operatorname{Mod}(S))$ and $P \in D(S)$. The complex $R \operatorname{Hom}_{S}(M, P)$ can be viewed as an object of $D(G-\operatorname{Mod}(S))$ by giving $M$ a right $G$-action by $m g:=g^{-1} m$ and $P$ a trivial $G$-action. We have

$$
\begin{aligned}
R \operatorname{Hom}_{G-\operatorname{Mod}(S)}\left(\mathbb{Z}, R \operatorname{Hom}_{S}(M, P)\right) & =R \operatorname{Hom}_{S}\left(M \otimes_{\mathbb{Z}[G]}^{L} \mathbb{Z}, P\right) \\
& =R \operatorname{Hom}_{S}\left(\mathbb{Z} \otimes_{\mathbb{Z}[G]}^{L} M, P\right)
\end{aligned}
$$

by the derived tensor-hom adjunction [KS06, Rmk. 18.6.11] and interchanging the tensor factors. In our notation, this means

$$
R \Gamma\left(G, R \operatorname{Hom}_{S}(M, P)\right)=R \operatorname{Hom}_{S}(L \Delta(G, M), P)
$$

(cf. the universal coefficient theorem). Hence the triangle (6.5) induces a distinguished triangle

$$
\begin{aligned}
R \operatorname{Hom}_{S}(R \hat{\Gamma}(G, M), P) & \rightarrow R \operatorname{Hom}_{S}(R \Gamma(G, M), P) \\
& \rightarrow R \Gamma\left(G, R \operatorname{Hom}_{S}(M, P)\right) .
\end{aligned}
$$

Proposition (6.8). Let $G$ be a finite group, $S$ a site and $M \in D^{b}(G-\operatorname{Mod}(S))$. Assume that $G$ is cyclic and $R \Gamma(G, M)$ is bounded. Then we have $R \hat{\Gamma}(G, M)=0$, and the norm map gives an isomorphism $L \Delta(G, M) \stackrel{\sim}{\rightarrow} R \Gamma(G, M)$ between homology

\footnotetext{
16 If $M$ is unbounded, the above definition of $R \hat{\Gamma}(G, M)$ does not factor through the derived category $D(G-\operatorname{Mod}(S))$ and hence is not "correct". In this case, we need to define $R \hat{\Gamma}(G, M)$ to be the mapping cone of the norm map $C(G) \otimes_{\mathbb{Z}[G]} I \rightarrow \operatorname{Hom}_{G-\operatorname{Mod}(S)}(C(G), I)$, where $M \stackrel{\sim}{\rightarrow} I$ is a K-injective replacement in $G-\operatorname{Mod}(S)$. Below we use bounded $M$ only.
} 
and cohomology. In particular, the triangle (6.7) for any $P \in D(S)$ reduces to an isomorphism

$$
R \operatorname{Hom}_{S}(R \Gamma(G, M), P)=R \Gamma\left(G, R \operatorname{Hom}_{S}(M, P)\right)
$$

in $D(S)$.

Proof. Let $\sigma$ be a generator of the cyclic group $G$. Then $\hat{C}(G)$ is chain homotopic

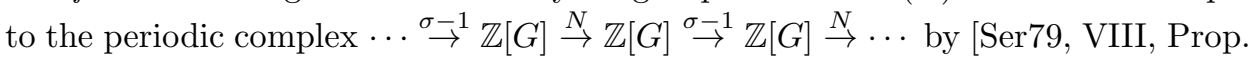
6] (see also [Bro82, VI, Prop. 3.3]). Therefore we have $R \hat{\Gamma}(G, M)=R \hat{\Gamma}(G, M)[2]$. On the other hand, the boundedness of $M$ and $R \Gamma(G, M)$ implies that $R \hat{\Gamma}(G, M)$ is acyclic in large degrees. Therefore $R \hat{\Gamma}(G, M)=0$. Hence $L \Delta(G, M) \stackrel{\sim}{\rightarrow} R \Gamma(G, M)$.

When $S=$ Spec $k_{\text {proet }}^{\text {indrat }}$, the last isomorphism in the above proposition for $P=\mathbb{Z}$ may be written as $R \Gamma(G, M)^{\mathrm{SD}}=R \Gamma\left(G, M^{\mathrm{SD}}\right)$.

Next we give a variant of the Hochschild-Serre spectral sequence. Let $L$ be a totally ramified (for simplicity) finite Galois extension of $K$ with Galois group $G$. For any $A \in \mathrm{Ab}\left(K_{\mathrm{fppf}} / k_{\mathrm{et}}^{\text {indrat }}\right)$ and $k^{\prime} \in k^{\text {indrat }}$, the $G$-action on $L$ induces a $G$-action on $\Gamma\left(\left(\mathbf{L}\left(k^{\prime}\right), k^{\prime}\right), A\right)$. Hence the functor $\boldsymbol{\Gamma}(L, \cdot): \operatorname{Ab}\left(K_{\text {fppf }} / k_{\text {et }}^{\text {indrat }}\right) \rightarrow \operatorname{Ab}\left(k_{\mathrm{et}}^{\text {indrat }}\right)$ factors through $G-\operatorname{Mod}\left(k_{\mathrm{et}}^{\text {indrat }}\right)$ and the functor $R \boldsymbol{\Gamma}(L, \cdot): D\left(K_{\mathrm{fppf}} / k_{\mathrm{et}}^{\text {indrat }}\right) \rightarrow$ $D\left(k_{\text {et }}^{\text {indrat }}\right)$ factors through $D\left(G-\operatorname{Mod}\left(k_{\text {et }}^{\text {indrat }}\right)\right)$. An object of $\operatorname{Ab}\left(k_{\text {et }}^{\text {indrat }}\right)$ can be viewed as an object of $G$-Mod $\left(k_{\text {et }}^{\text {indrat }}\right)$ by putting the trivial $G$-action. The resulting functor $D\left(k_{\mathrm{et}}^{\text {indrat }}\right) \rightarrow D\left(G\right.$-Mod $\left.\left(k_{\mathrm{et}}^{\text {indrat }}\right)\right)$ is left adjoint to the derived $G$-invariants $R \Gamma(G, \cdot)$. For any $A \in D\left(K_{\mathrm{fppf}} / k_{\mathrm{et}}^{\text {indrat }}\right)$, the natural morphism $R \boldsymbol{\Gamma}(K, A) \rightarrow$ $R \boldsymbol{\Gamma}(L, A)$ in $D\left(k_{\mathrm{et}}^{\text {indrat }}\right)$ factors through $R \Gamma(G, R \boldsymbol{\Gamma}(L, A))$ by adjunction. Similarly, the inclusion $R \tilde{\boldsymbol{\Gamma}}(K, A) \rightarrow R \tilde{\boldsymbol{\Gamma}}(L, A)$ in $D\left(k_{\text {proet }}^{\text {indrat }}\right)$ factors through $R \Gamma(G, R \tilde{\boldsymbol{\Gamma}}(L, A))$.

Proposition (6.9). Let $L$ be a totally ramified finite Galois extension of $K$ with Galois group $G$. Let $A \in D\left(K_{\mathrm{fppf}} / k_{\mathrm{et}}^{\text {indrat }}\right)$. The morphism

$$
R \Gamma(G, R \boldsymbol{\Gamma}(L, A)) \leftarrow R \boldsymbol{\Gamma}(K, A)
$$

in $D\left(k_{\mathrm{et}}^{\text {indrat }}\right)$ defined above is an isomorphism. The morphism

$$
R \Gamma(G, R \tilde{\boldsymbol{\Gamma}}(L, A)) \leftarrow R \tilde{\boldsymbol{\Gamma}}(K, A)
$$

in $D\left(k_{\text {proet }}^{\text {indrat }}\right)$ defined above is an isomorphism if $A$ is bounded below.

Proof. We first treat the first morphism. For any $k^{\prime} \in k^{\text {indrat }}$, the right-hand side after applying $R \Gamma\left(k_{\text {et }}^{\prime}, \cdot\right)$ is $R \Gamma\left(\mathbf{K}\left(k^{\prime}\right), A\right)$, where we view $A$ as an fppf sheaf on $\operatorname{Spec} \mathbf{K}\left(k^{\prime}\right)$ as in (3.3.1). The left-hand side after applying $R \Gamma\left(k_{\text {et }}^{\prime}, \cdot\right)$ is

$$
\begin{aligned}
R \Gamma\left(k_{\mathrm{et}}^{\prime}, R \Gamma(G, R \boldsymbol{\Gamma}(L, A))\right) & =R \Gamma\left(G, R \Gamma\left(k_{\mathrm{et}}^{\prime}, R \boldsymbol{\Gamma}(L, A)\right)\right) \\
& =R \Gamma\left(G, R \Gamma\left(\mathbf{L}\left(k^{\prime}\right), A\right)\right),
\end{aligned}
$$

where the $R \Gamma(G, \cdot)$ in the second and third terms are the usual group cohomology. Hence the first morphism in the statement after applying $R \Gamma\left(k_{\text {et }}^{\prime}, \cdot\right)$ is

$$
R \Gamma\left(G, R \Gamma\left(\mathbf{L}\left(k^{\prime}\right), A\right)\right) \leftarrow R \Gamma\left(\mathbf{K}\left(k^{\prime}\right), A\right)
$$

in $D(\mathrm{Ab})$. This is an isomorphism by the usual Hochschild-Serre spectral sequence Mil80, III, Rmk. 2.21 (a)] since the morphism $\operatorname{Spec} \mathbf{L}\left(k^{\prime}\right) \rightarrow \operatorname{Spec} \mathbf{K}\left(k^{\prime}\right)$ is a $G$ covering. This implies that the first morphism in the proposition is an isomorphism. 
For the second, it is enough to show that $R \Gamma(G, M)^{\sim}=R \Gamma(G, \tilde{M})$ if $M \in$ $D^{+}\left(G-\operatorname{Mod}\left(k_{\text {et }}^{\text {indrat }}\right)\right)$, where $\sim$ denotes pro-étale sheafification. By (6.3) (which does not require $M$ to be bounded above), the $n$-th term of $R \Gamma(G, M)$ is given by

$$
\prod_{i+j=n} \operatorname{Hom}_{k_{\mathrm{et}}^{\text {indrat }}}\left(C(G)^{-i}, M^{j}\right)=\prod_{i+j=n} C^{\vee}(G)^{i} \otimes_{\mathbb{Z}[G]} M^{j} .
$$

The final product is a finite product since $C^{\vee}(G)$ and $M$ are bounded below. Hence the $n$-th term of $R \Gamma(G, M)^{\sim}$ is

$$
\prod_{i+j=n} \mathbf{H o m}_{k_{\mathrm{proet}}^{\text {indrat }}}\left(C(G)^{-i}, \tilde{M}^{j}\right),
$$

which is the $n$-th term of $R \Gamma(G, \tilde{M})$.

Proof of (6.1). Let $A$ be an abelian variety over $K$. Assume that (4.1.2) is true for $A$ over a finite Galois extension $L$ of $K$ :

$$
\theta_{A \times{ }_{K} L}: R \boldsymbol{\Gamma}\left(L, A^{\vee}\right)^{\mathrm{SDSD}} \stackrel{\sim}{\rightarrow} R \boldsymbol{\Gamma}(L, A)^{\mathrm{SD}} .
$$

in $D\left(k_{\text {proet }}^{\text {indrat }}\right)$, where $k^{\prime}$ is the residue field of $L$. We want to deduce the corresponding statement for $A$ over $K$. If $L / K$ is unramified, then the morphism above for $A$ over $L$ is nothing but the morphism for $A$ over $K$ restricted from $D\left(k_{\text {proet }}^{\text {indrat }}\right)$ to $D\left(k_{\text {proet }}^{\text {indrat }}\right)$. Therefore the invertibility of these morphisms is equivalent. Hence we may assume that $L / K$ is totally ramified. Since it is a solvable extension Ser79, IV, Cor. 5 to Prop. 7], we may further assume that $L / K$ is cyclic.

Let $G=\operatorname{Gal}(L / K)$. As we saw, the complexes $R \Gamma(L, A)$ and $R \Gamma\left(L, A^{\vee}\right)$ may be viewed as objects of $D\left(G-\operatorname{Mod}\left(k_{\text {proet }}^{\text {indrat }}\right)\right)$. We show that the morphism $\theta_{A \times{ }_{K} L}$ is $G$-equivariant, i.e. a morphism in $D\left(G-\operatorname{Mod}\left(k_{\text {proet }}^{\text {indrat }}\right)\right)$. From the construction of the (normalized) functorial valuation map $v_{L}: \mathbf{L}^{\times} \rightarrow \mathbb{Z}$ for $L$ given in the paragraph before [Suz13, Prop. 2.4.4], we see that $v_{L}$ is $G$-equivariant, where we put a trivial $G$-action on $\mathbb{Z}$. Recall from $\$ 4.1$ that the morphism $\theta_{A \times_{K} L}$ is defined as the Serre dual of the composite

$$
\begin{aligned}
R \boldsymbol{\Gamma}(L, A) & \rightarrow R \tilde{\boldsymbol{\Gamma}}\left(L, R \mathbf{H o m}_{L}\left(A^{\vee}, \mathbf{G}_{m}\right)\right)[1] \\
& \rightarrow R \mathbf{H o m}_{k_{\text {proet }}^{\text {indrat }}}\left(R \boldsymbol{\Gamma}\left(L, A^{\vee}\right), R \boldsymbol{\Gamma}\left(L, \mathbf{G}_{m}\right)\right)[1] \\
& \rightarrow R \mathbf{H o m}_{k_{\text {proet }}^{\text {indrat }}}\left(R \boldsymbol{\Gamma}\left(L, A^{\vee}\right), \mathbb{Z}\right)[1]=R \boldsymbol{\Gamma}\left(L, A^{\vee}\right)^{\mathrm{SD}}[1] .
\end{aligned}
$$

All these morphisms, including the trace morphism $R \Gamma\left(L, \mathbf{G}_{m}\right)=\mathbf{L}^{\times} \stackrel{v_{L}}{\rightarrow} \mathbb{Z}$ over $L$, are $G$-equivariant.

We apply $R \Gamma(G, \cdot)$ to $\theta_{A \times_{K} L}$. We have

$$
R \Gamma(G, R \Gamma(L, A))=R \Gamma(K, A)
$$

in $D\left(k_{\text {proet }}^{\text {indrat }}\right)$ by the Hochschild-Serre spectral sequence (6.9). The both complexes $R \boldsymbol{\Gamma}(L, A)$ and $R \boldsymbol{\Gamma}(K, A)$ are concentrated in degrees 0 and 1 . Hence we can apply (6.8) for $M=R \boldsymbol{\Gamma}(L, A)$ and $P=\mathbb{Z}$. This yields

$$
R \Gamma\left(G, R \boldsymbol{\Gamma}(L, A)^{\mathrm{SD}}\right)=R \boldsymbol{\Gamma}(K, A)^{\mathrm{SD}} .
$$

The both complexes $R \boldsymbol{\Gamma}(L, A)^{\mathrm{SD}}$ and $R \boldsymbol{\Gamma}(K, A)^{\mathrm{SD}}$ are concentrated in degrees 0 to 2. Applying the same proposition again, we have

$$
R \Gamma\left(G, R \boldsymbol{\Gamma}(L, A)^{\mathrm{SDSD}}\right)=R \boldsymbol{\Gamma}(K, A)^{\mathrm{SDSD}} .
$$


Now we apply $R \Gamma(G, \cdot)$ to the both sides of $\theta_{A \times_{K} L}$ to get an isomorphism

$$
\theta_{A \times_{K} L}^{G}: R \boldsymbol{\Gamma}\left(K, A^{\vee}\right)^{\mathrm{SDSD}} \stackrel{\sim}{\rightarrow} R \boldsymbol{\Gamma}(K, A)^{\mathrm{SD}} .
$$

We show that this isomorphism $\theta_{A \times{ }_{K} L}^{G}$ is equal to $\theta_{A}$. Consider the morphism

$$
R \boldsymbol{\Gamma}\left(L, A^{\vee}\right) \otimes^{L} R \boldsymbol{\Gamma}(L, A) \rightarrow R \boldsymbol{\Gamma}\left(L, \mathbf{G}_{m}\right)[1]
$$

in $D\left(G-\operatorname{Mod}\left(k_{\text {proet }}^{\text {indrat }}\right)\right)$. Apply $R \Gamma(G, \cdot)$. The same proof as Suz13, Prop. 2.4.3 and the paragraph after] shows that there is a cup product pairing

$$
R \Gamma\left(G, R \boldsymbol{\Gamma}\left(L, A^{\vee}\right)\right) \otimes^{L} R \Gamma(G, R \boldsymbol{\Gamma}(L, A)) \rightarrow R \Gamma\left(G, R \boldsymbol{\Gamma}\left(L, \mathbf{G}_{m}\right)\right)[1],
$$

which can be identified with

$$
R \boldsymbol{\Gamma}\left(K, A^{\vee}\right) \otimes^{L} R \boldsymbol{\Gamma}(K, A) \rightarrow R \boldsymbol{\Gamma}\left(K, \mathbf{G}_{m}\right)[1] .
$$

This and the trace morphism $R \boldsymbol{\Gamma}\left(K, \mathbf{G}_{m}\right)[1]=\mathbf{K}^{\times} \stackrel{v_{K}}{\rightarrow} \mathbb{Z}[1]$ for $K$ leads to the morphism $\theta_{A}$ via the derived tensor-hom adjunction (used before). On the other hand, (6.10) gives morphisms

$$
\begin{aligned}
R \boldsymbol{\Gamma}\left(L, A^{\vee}\right) & \rightarrow R \mathbf{H o m}_{k_{\text {proet }}^{\text {indrat }}}\left(R \boldsymbol{\Gamma}(L, A), R \boldsymbol{\Gamma}\left(L, \mathbf{G}_{m}\right)\right)[1] \\
& \rightarrow R \mathbf{H o m}_{k_{\text {proet }}^{\text {indrat }}}\left(R \boldsymbol{\Gamma}(L, A), L \Delta\left(G, R \boldsymbol{\Gamma}\left(L, \mathbf{G}_{m}\right)\right)\right)[1]
\end{aligned}
$$

in $D\left(G-\operatorname{Mod}\left(k_{\text {proet }}^{\text {indrat }}\right)\right)$, where the second morphism is the natural morphism (i.e. $M \rightarrow \mathbb{Z} \otimes_{\mathbb{Z}[G]}^{L} M$ given by $\left.m \mapsto 1 \otimes m\right)$ and $L \Delta\left(G, R \boldsymbol{\Gamma}\left(L, \mathbf{G}_{m}\right)\right)$ here is given the trivial $G$-action. Applying $R \Gamma(G, \cdot)$ and using (6.6) for $P=L \Delta\left(G, R \boldsymbol{\Gamma}\left(L, \mathbf{G}_{m}\right)\right)$, we have a morphism

$$
\begin{aligned}
& R \Gamma\left(G, R \boldsymbol{\Gamma}\left(L, A^{\vee}\right)\right) \\
& \rightarrow R \mathbf{H o m}_{k_{\text {proet }}^{\text {indrat }}}\left(L \Delta(R \boldsymbol{\Gamma}(L, A)), L \Delta\left(G, R \boldsymbol{\Gamma}\left(L, \mathbf{G}_{m}\right)\right)\right)[1] .
\end{aligned}
$$

By the derived tensor-hom adjunction, we have a morphism

$$
R \Gamma\left(G, R \boldsymbol{\Gamma}\left(L, A^{\vee}\right)\right) \otimes^{L} L \Delta(G, R \boldsymbol{\Gamma}(L, A)) \rightarrow L \Delta\left(G, R \boldsymbol{\Gamma}\left(L, \mathbf{G}_{m}\right)\right)[1] .
$$

This and the trace morphism $L \Delta\left(G, R \boldsymbol{\Gamma}\left(L, \mathbf{G}_{m}\right)\right)=L \Delta\left(G, \mathbf{L}^{\times}\right) \stackrel{v_{L}}{\rightarrow} \mathbb{Z}$ for $L$ leads to the morphism $\theta_{A \times_{K} L}^{G}$. The two morphisms (6.11) and (6.12) and the two trace morphisms are compatible:

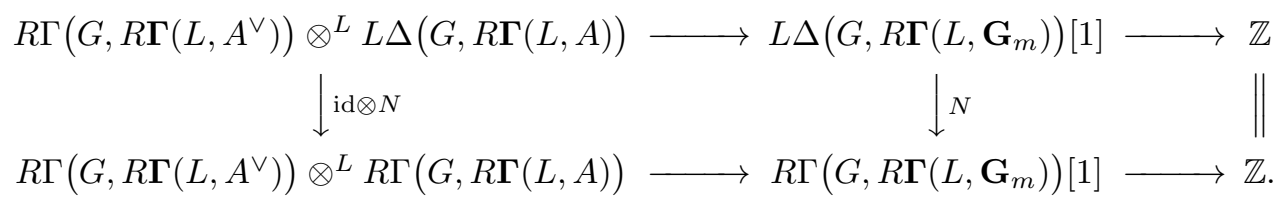

The commutativity of the left square is trivial. That of the right comes from the equality $v_{L}=v_{K} \circ N: \mathbf{L}^{\times} \rightarrow \mathbf{K}^{\times} \rightarrow \mathbb{Z}$ of the normalized valuations of $K$ and $L$, which is true as $L / K$ is totally ramified. This implies $\theta_{A}=\theta_{A \times_{K} L}^{G}$. Hence the invertibility of $\theta_{A \times_{K} L}$ implies that of $\theta_{A}$.

Remark (6.13).

(a) The assumptions in (6.8) can be weakened to make it parallel to Nakayama's theorem ([Ser79, IX, $\S 5$, Thm. 8]). The group $G$ may be any finite group. The complex $M$ may just be bounded, with no restrictions on the number of non-zero terms or their positions. The assumption for $R \hat{\Gamma}(G, M)$ should now be that for any prime number $l$ and an $l$-Sylow subgroup $G_{l}$ of $G$, the 
complex $R \hat{\Gamma}\left(G_{l}, M\right)$ be acyclic in two consecutive degrees. The conclusion is that $R \hat{\Gamma}(H, M)=0$ for any subgroup $H$ of $G$. To prove this, first notice that the statement is reduced to the corresponding statement for a complex of usual $G$-modules (not sheaves of $G$-modules), by considering the Homcomplex $\operatorname{Hom}_{k_{\text {proet }}^{\text {indrat }}}(M, I)$ for any injective sheaf $I \in \mathrm{Ab}\left(k_{\text {proet }}^{\text {indrat }}\right)$. Then the statement was proved by Koya Koy93, Prop. 4]. Alternatively, we may assume that $M$ is concentrated in degree zero, since the bounded derived category of $\mathbb{Z}[G]$-modules modulo bounded perfect complexes and the stable category of $\mathbb{Z}[G]$-modules are equivalent ([BIK13, Prop. 8.2]). Then the statement is equivalent to Nakayama's theorem.

(b) (5.2.1) is true without assuming $A$ to be semistable. We can see this by the following Galois descent argument. Assume that $k$ is algebraically closed and $A$ has semistable reduction over a finite Galois extension $L$ of $K$. Let $G=\operatorname{Gal}(L / K)$. Let $\mathcal{A}_{L}$ be the Néron model of $A_{L}=A \times_{K} L$. The group $\mathcal{A}_{L}$ has a $G$-equivariant sheaf structure coming from the descent data of $A_{L}$. Therefore, for an integer $n \geq 1$ that kills $\pi_{0}\left(\left(\mathcal{A}_{L}\right)_{x}\right)$, the finite part $\mathcal{A}_{L}[n]^{f}$ of the $n$-torsion part of $\mathcal{A}_{L}$ also has a $G$-equivariant sheaf structure. Since the pairing $\left(\mathcal{A}_{L}[n]^{f}\right)^{\mathrm{CD}} \times_{\mathcal{O}_{L}} \mathcal{A}_{L}[n]^{f} \rightarrow \mathbf{G}_{m}$ is $G$-equivariant and the trace isomorphism $R \boldsymbol{\Gamma}_{x}\left(\mathcal{O}_{L}, \mathbf{G}_{m}\right)=\mathbb{Z}[-1]$ is $G$-invariant, the isomorphism

$$
R \boldsymbol{\Gamma}_{x}\left(\mathcal{O}_{K},\left(\mathcal{A}_{L}[n]^{f}\right)^{\mathrm{CD}}\right) \rightarrow R \boldsymbol{\Gamma}\left(\mathcal{O}_{K}, \mathcal{A}_{L}[n]^{f}\right)^{\mathrm{SD}}[-1]
$$

in (5.2.1.2) is an isomorphism in $D\left(G-\operatorname{Mod}\left(k_{\text {proet }}^{\text {indrat }}\right)\right)$. From this, we can see that all the steps in the construction of Bertapelle's isomorphism

$$
\psi_{A_{L}}: H^{1}\left(L, A^{\vee}\right) \stackrel{\sim}{\rightarrow} \operatorname{Ext}_{k}^{1}(\boldsymbol{\Gamma}(L, A), \mathbb{Q} / \mathbb{Z})
$$

is $G$-equivariant. Hence $\psi_{A_{L}}$ itself is $G$-equivariant. For $n \geq 0$, let $L^{\otimes n}$ be the tensor product of $n$ copies of $L$ over $K$ and $\operatorname{Res}_{L \otimes n}{ }^{\otimes n}$ be the Weil restriction functor. Then we have an exact sequence

$$
0 \rightarrow A \rightarrow \operatorname{Res}_{L / K} A \rightarrow \operatorname{Res}_{L \otimes 2 / K} A \rightarrow \operatorname{Res}_{L \otimes 3 / K} A \rightarrow \cdots
$$

of abelian varieties over $K$ coming from the Čech complex (cf. the proof of [Mil80, III, Thm. 3.9]). The cokernel of any morphism in the sequence is an abelian variety. Dualizing, we have an exact sequence

$$
\cdots \rightarrow \operatorname{Res}_{L^{\otimes 3} / K} A^{\vee} \rightarrow \operatorname{Res}_{L^{\otimes 2} / K} A^{\vee} \rightarrow \operatorname{Res}_{L / K} A^{\vee} \rightarrow A^{\vee} \rightarrow 0
$$

of abelian varieties over $K$. Since $H^{2}(K$, abelian variety $)=0$, this induces an exact sequence

$$
H^{1}\left(K, \operatorname{Res}_{L \otimes 2} / K A^{\vee}\right) \rightarrow H^{1}\left(K, \operatorname{Res}_{L / K} A^{\vee}\right) \rightarrow H^{1}\left(K, A^{\vee}\right) \rightarrow 0,
$$

or

$$
\prod_{\sigma \in G} H^{1}\left(L, A^{\vee}\right) \stackrel{\prod \sigma}{\longrightarrow} H^{1}\left(L, A^{\vee}\right) \stackrel{\text { Cores }}{\longrightarrow} H^{1}\left(K, A^{\vee}\right) \rightarrow 0 .
$$

Hence the corestriction identifies $H^{1}\left(K, A^{\vee}\right)$ as the $G$-coinvariants of $H^{1}\left(L, A^{\vee}\right)$. Similarly, by (2.4.1) (国), the inclusion $\boldsymbol{\Gamma}(K, A) \hookrightarrow \boldsymbol{\Gamma}(L, A)$ identifies $\operatorname{Ext}_{k}^{1}(\boldsymbol{\Gamma}(K, A), \mathbb{Q} / \mathbb{Z})$ as the $G$-coinvariants of $\operatorname{Ext}_{k}^{1}(\boldsymbol{\Gamma}(L, A), \mathbb{Q} / \mathbb{Z})$. The construction of Bertapelle's isomorphism

$$
\psi_{A}: H^{1}\left(K, A^{\vee}\right) \stackrel{\sim}{\rightarrow} \operatorname{Ext}_{k_{\text {proet }}^{\text {indrat }}}^{1}(\boldsymbol{\Gamma}(K, A), \mathbb{Q} / \mathbb{Z})
$$


given in [Ber03, Thm. 3] is the same as to take the $G$-coinvariants of $\psi_{A_{L}}$. The same is true for our morphisms $\theta_{A_{L}}^{+1}(k)$ and $\theta_{A}^{+1}(k)$ by the functoriality of our constructions. Hence the equality $\theta_{A_{L}}^{+1}(k)=\psi_{A_{L}}$ implies the equality $\theta_{A}^{+1}(k)=\psi_{A}$.

\section{End of PRoOf: GRothendeCK's CONJECTURE}

Now we prove (4.1.2) and Grothendieck's conjecture by summarizing what we have done so far. Recall from Introduction that Šafarevič's conjecture, proved by Bégueri Bég81, Thm. 8.3.6], Bester [Bes78, §2.7, Thm. 7.1] and Bertapelle Ber03, Thm. 3], states that there exists a canonical isomorphism

$$
H^{1}\left(A^{\vee}\right) \rightarrow \operatorname{Ext}_{k}^{1}(\boldsymbol{\Gamma}(A), \mathbb{Q} / \mathbb{Z})
$$

for an abelian variety $A$ over $K$ when $k$ is algebraically closed. To be clear, in the proposition, the theorem and their proofs below, we mean by "Šafarevič's conjecture for $A$ " the statement that the morphism $\theta_{A}^{+1}$ in (4.2.1) is an isomorphism.

Proposition (7.1). Let $A$ be an abelian variety over $K$. Then (4.1.2) for $A$ is equivalent to the conjunction of the following three statements:

- Grothendieck's conjecture for A,

- Šafarevič's conjecture for $A \times_{K} \mathbf{K}\left(k^{\prime}\right)$ for any algebraically closed field $k^{\prime} \in$ $k^{\text {indrat }}$, and

- Šafarevič's conjecture for $A^{\vee} \times_{K} \mathbf{K}\left(k^{\prime}\right)$ for any algebraically closed field $k^{\prime} \in k^{\text {indrat }}$.

Proof. Immediate from (4.2.1) and (5.1.1).

Theorem (7.2). (4.1.2) and Grothendieck's conjecture are both true for any abelian variety $A$ over $K$. That is, the morphism

$$
\theta_{A}: R \boldsymbol{\Gamma}\left(A^{\vee}\right)^{\mathrm{SDSD}} \rightarrow R \boldsymbol{\Gamma}(A)^{\mathrm{SD}}
$$

in $D(k)$ is an isomorphism, and Grothendieck's pairing

$$
\pi_{0}\left(\mathcal{A}_{x}^{\vee}\right) \times \pi_{0}\left(\mathcal{A}_{x}\right) \rightarrow \mathbb{Q} / \mathbb{Z}
$$

is perfect.

Proof. By the semistable reduction theorem, there exists a finite Galois extension $L / K$ such that $A$ has semistable reduction over $L$. Then Grothendieck's conjecture is true for $A$ over $L$ by Werner's result Wer97. Also Šafarevič's conjecture is true for $A$ over $L$ by (5.2.1) and (5.3.1) and the results of Bégueri, Bester and Bertapelle cited above. Hence (4.1.2) is true for $A$ over $L$ by the previous proposition. Hence it is true for $A$ over $K$ by (6.1). Hence Grothendieck's conjecture is true for $A$ over $K$ by the previous proposition.

This completes the proof of Theorems A, B and C.

Remark (7.3). Since $R \boldsymbol{\Gamma}\left(A^{\vee}\right)$ is $\mathrm{P}$-acyclic, we know that its $R \Gamma\left(k_{\text {proet }}, \cdot\right)$ is the usual cohomology complex $R \Gamma\left(A^{\vee}\right)=R \Gamma\left(K, A^{\vee}\right)$. Therefore the duality

$$
\left[\stackrel{\lim }{n}_{n} \boldsymbol{\Gamma}\left(A^{\vee}\right) \rightarrow R \boldsymbol{\Gamma}\left(A^{\vee}\right)\right] \stackrel{\sim}{\rightarrow} R \mathbf{H o m}_{k_{\text {proet }}^{\text {indrat }}}(R \boldsymbol{\Gamma}(A), \mathbb{Q} / \mathbb{Z}),
$$

true in $D\left(k_{\text {proet }}^{\text {indrat }}\right)$, gives a statement about $R \Gamma\left(A^{\vee}\right)$ by taking the $R \Gamma\left(k_{\text {proet }}, \cdot\right)$ of the both sides, even if $k$ is not algebraically closed. 
To make this explicit, let $\left(\mathcal{A}_{x}^{\vee}\right)_{\mathrm{SAb}}$ be the maximal semi-abelian quotient of $\left(\mathcal{A}_{x}^{\vee}\right)_{0}$. and $\overline{\left(\mathcal{A}_{x}^{\vee}\right)_{\mathrm{sAb}}}$ its universal covering. Then

$$
\lim _{n} \Gamma\left(A^{\vee}\right)={\underset{\swarrow}{n}}_{\lim _{x}}\left(\mathcal{A}_{x}^{\vee}\right)_{\mathrm{sAb}}=\overline{\left(\mathcal{A}_{x}^{\vee}\right)_{\mathrm{sAb}}}
$$

by (2.4.1) (d), where $\lim _{n}$ denotes the inverse limit for multiplication by $n \geq 1$. These groups do not change under replacing $\lim _{n}$ by $R{\underset{\leftarrow}{\longleftarrow}}_{n}$ by (2.1.2) (f). We have

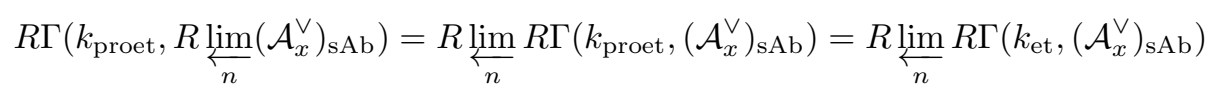

by (2.2.4) (b) and (2.1.2) (g). Hence

$$
R \Gamma\left(k_{\text {proet }},{\underset{\leftarrow}{n}}_{\lim _{n}} \Gamma\left(A^{\vee}\right)\right)=R \Gamma\left(k_{\text {proet }}, \overline{\left(\mathcal{A}_{x}^{\vee}\right)_{\mathrm{sAb}}}\right)=R{\underset{\leftarrow}{n}}_{\lim _{n}} R \Gamma\left(k_{\text {et }},\left(\mathcal{A}_{x}^{\vee}\right)_{\mathrm{sAb}}\right) .
$$

Denote these isomorphic objects by $Q_{k, A^{\vee}}$. It is a complex of $\mathbb{Q}$-vector spaces. We have

$$
\begin{aligned}
R \Gamma\left(k_{\text {proet }}, R \mathbf{H o m}_{k_{\text {proet }}^{\text {ind rat }}}(R \boldsymbol{\Gamma}(A), \mathbb{Q} / \mathbb{Z})\right) & =R \operatorname{Hom}_{k_{\text {proet }}^{\text {indrat }}}(R \boldsymbol{\Gamma}(A), \mathbb{Q} / \mathbb{Z}) \\
& =R \operatorname{Hom}_{\mathrm{IPAlg} / k}(R \boldsymbol{\Gamma}(A), \mathbb{Q} / \mathbb{Z})
\end{aligned}
$$

by (2.3.3). Therefore the duality isomorphism in $D\left(k_{\text {proet }}^{\text {indrat }}\right)$ above gives an isomorphism

$$
\left[Q_{k, A^{\vee}} \rightarrow R \Gamma\left(A^{\vee}\right)\right] \stackrel{\sim}{\rightarrow} R \operatorname{Hom}_{\mathrm{IPAlg} / k}(R \Gamma(A), \mathbb{Q} / \mathbb{Z})
$$

in $D(\mathrm{Ab})$.

\section{Duality With COefFicients in tori}

In this section, we give an analogue of (4.1.2) for tori and describe it. In this and the next sections, we write $\mathbf{H o m}_{k}=\mathbf{H o m}_{k_{\text {proet }}^{\text {indrat }}}$ and similarly for Ext and $R$ Hom, and $R \boldsymbol{\Gamma}(\cdot)=R \boldsymbol{\Gamma}(K, \cdot)$, when there is no confusion.

Let $T$ be a torus over $K$ with Néron model $\mathcal{T}$ and Cartier dual $T^{\mathrm{CD}}$. Let $\mathcal{T}_{x}$ be the special fiber of $\mathcal{T}$. By (3.4.3) (远), we know that $\boldsymbol{\Gamma}(T)$ is an extension of an étale group by a P-acyclic proalgebraic group, $\mathbf{H}^{n}(T)=0$ for $n \geq 1$, and $R \boldsymbol{\Gamma}(T)=\boldsymbol{\Gamma}(T)$ is $\mathrm{P}$-acyclic. Also by (3.4.3) ((C), $\boldsymbol{\Gamma}\left(T^{\mathrm{CD}}\right)$ is a lattice over $k, \mathbf{H}^{1}\left(T^{\mathrm{CD}}\right) \in \mathrm{FEt} / k$, $\mathbf{H}^{2}\left(T^{\mathrm{CD}}\right) \in \mathrm{IAlg}_{\mathrm{uc}} / k, \mathbf{H}^{n}\left(T^{\mathrm{CD}}\right)=0$ for $n \geq 3$, and $R \boldsymbol{\Gamma}\left(T^{\mathrm{CD}}\right)$ is $\mathrm{P}$-acyclic and Serre reflexive. Therefore we will write $R \tilde{\boldsymbol{\Gamma}}(T)=R \boldsymbol{\Gamma}(T)$ and $R \tilde{\boldsymbol{\Gamma}}\left(T^{\mathrm{CD}}\right)=R \boldsymbol{\Gamma}\left(T^{\mathrm{CD}}\right)$. The morphism of functoriality of $R \tilde{\boldsymbol{\Gamma}}$ (3.3.8) and the trace morphism $R \boldsymbol{\Gamma}\left(\mathbf{G}_{m}\right) \rightarrow \mathbb{Z}$ (4.1.1) yield morphisms

$$
\begin{aligned}
R \boldsymbol{\Gamma}\left(T^{\mathrm{CD}}\right) & \rightarrow R \tilde{\boldsymbol{\Gamma}} R \mathbf{H o m}_{K}\left(T, \mathbf{G}_{m}\right) \\
& \rightarrow R \mathbf{H o m}_{k}\left(R \boldsymbol{\Gamma}(T), R \boldsymbol{\Gamma}\left(\mathbf{G}_{m}\right)\right) \\
& \rightarrow R \mathbf{H o m}_{k}(R \boldsymbol{\Gamma}(T), \mathbb{Z})=R \boldsymbol{\Gamma}(T)^{\mathrm{SD}}
\end{aligned}
$$

in $D\left(k_{\text {proet }}^{\text {indrat }}\right)$. The following is the duality with coefficients in tori. See also Bég81, $7.2]$ in the case of mixed characteristic $K$ with algebraically closed $k$.

Theorem (8.1). The morphism

$$
\theta_{T}: R \boldsymbol{\Gamma}\left(T^{\mathrm{CD}}\right) \rightarrow R \boldsymbol{\Gamma}(T)^{\mathrm{SD}}
$$


in $D\left(k_{\text {proet }}^{\text {indrat }}\right)$ defined above is an isomorphism 17 Its Serre dual yields isomorphisms

$$
\theta_{T} \mathrm{CD}:\left[\varliminf_{n} \boldsymbol{\Gamma}(T) \rightarrow R \boldsymbol{\Gamma}(T)\right]=R \boldsymbol{\Gamma}(T)^{\mathrm{SDSD}}=R \boldsymbol{\Gamma}\left(T^{\mathrm{CD}}\right)^{\mathrm{SD}} .
$$

Proof. We first treat $\theta_{T}$. If $T=\mathbf{G}_{m}$, then it is an isomorphism by Suz13, Thm. 2.6.1] (which is essentially Serre's local class field theory Ser61]). For general $T$, let $L / K$ be a finite Galois extension with Galois group $G$ that splits $T$, so that we have

$$
\theta_{T \times_{K} L}: R \boldsymbol{\Gamma}\left(L, T^{\mathrm{CD}}\right) \stackrel{\sim}{\rightarrow} R \boldsymbol{\Gamma}(L, T)^{\mathrm{SD}} .
$$

We may assume that $L / K$ is totally ramified and cyclic as in the proof of (6.1). The complexes $R \boldsymbol{\Gamma}(L, T)$ and

$$
R \Gamma(G, R \Gamma(L, T))=R \Gamma(K, T)
$$

are both concentrated in degree zero. Therefore we can apply (6.8), obtaining

$$
R \Gamma\left(G, R \boldsymbol{\Gamma}(L, T)^{\mathrm{SD}}\right)=R \boldsymbol{\Gamma}(K, T)^{\mathrm{SD}} .
$$

Applying $R \Gamma(G, \cdot)$ to $\theta_{T \times_{K} L}$, we obtain an isomorphism $R \boldsymbol{\Gamma}\left(K, T^{\mathrm{CD}}\right) \stackrel{\sim}{\rightarrow} R \boldsymbol{\Gamma}(K, T)^{\mathrm{SD}}$. We can see that this isomorphism is equal to $\theta_{T}$ by the same method as the last part of the proof of (6.1).

Before treating $\theta_{T^{\mathrm{CD}}}$, we show that $\pi_{0}\left(\mathcal{T}_{x}\right)$ is a finitely generated étale group over $k$. This is well-known (see [HN11, Prop. 3.5] for example), but can be deduced as a corollary of the method of proof in the previous paragraph, as follows. By (6.8), we know that the norm map gives an isomorphism

$$
L \Delta(G, \boldsymbol{\Gamma}(L, T)) \stackrel{N}{\rightarrow} R \Gamma(G, \Gamma(L, T))=\boldsymbol{\Gamma}(K, T)
$$

if $L / K$ is finite Galois totally ramified and cyclic. The cyclicity can be removed by dévissage. Since the zeroth group homology is the coinvariants, we know that the $G$-coinvariants of $\boldsymbol{\Gamma}(L, T)$ is $\boldsymbol{\Gamma}(K, T)$. The group $\pi_{0}(\boldsymbol{\Gamma}(K, T))$ is the Néron component group of $T$. Hence the $G$-coinvariants of the Néron component group of $T \times_{K} L$ is the Néron component group of $T$. Since the Néron component group of a split torus is obviously finite free, this implies that $\pi_{0}\left(\mathcal{T}_{x}\right)$ is finitely generated.

For $\theta_{T_{\mathrm{CD}}}$, recall again that $R \boldsymbol{\Gamma}(T)=\boldsymbol{\Gamma}(T)$. Hence the only non-trivial part (after the invertibility of $\theta_{T}$ shown above) is

$$
\boldsymbol{\Gamma}(T)^{\mathrm{SDSD}}=\left[\lim _{n} \boldsymbol{\Gamma}(T) \rightarrow \boldsymbol{\Gamma}(T)\right] .
$$

Let $\boldsymbol{\Gamma}(T)_{0} \in \mathrm{PAlg} / k$ be the identity component of $\boldsymbol{\Gamma}(T)$. By (2.4.1) (d), we have

$$
\boldsymbol{\Gamma}(T)_{0}^{\mathrm{SDSD}}=\left[\lim _{n} \boldsymbol{\Gamma}(T)_{0} \rightarrow \boldsymbol{\Gamma}(T)_{0}\right] \in D^{b}\left(\mathrm{PAlg}_{\mathrm{uc}}\right) .
$$

Since $\pi_{0}(\boldsymbol{\Gamma}(T))$ is finitely generated, we have $\lim _{n} \boldsymbol{\Gamma}(T)=\lim _{n} \boldsymbol{\Gamma}(T)_{0}$, which is a uniquely divisible proalgebraic group. Hence

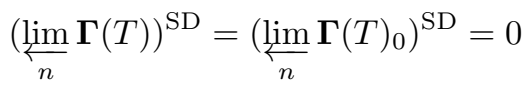

as seen in the proof of (2.4.1) (d). Therefore

$$
\boldsymbol{\Gamma}(T)^{\mathrm{SDSD}}=\left[{\underset{n}{n}}_{\lim } \boldsymbol{\Gamma}(T) \rightarrow \boldsymbol{\Gamma}(T)\right]^{\mathrm{SDSD}} .
$$

\footnotetext{
${ }^{17}$ Since $R \boldsymbol{\Gamma}\left(T^{\mathrm{CD}}\right)$ is Serre reflexive as we saw above, there is no point putting SDSD to it and using a different letter $\vartheta_{T}$ to denote this morphism, as opposed to the case of abelian varieties.
} 
We do not need SDSD on the right-hand side, since the distinguished triangle

$$
\left[\stackrel{\lim }{n}_{n} \boldsymbol{\Gamma}(T)_{0} \rightarrow \boldsymbol{\Gamma}(T)_{0}\right] \rightarrow\left[\overleftarrow{n}_{n} \lim _{\mathbf{m}} \boldsymbol{\Gamma}(T) \rightarrow \boldsymbol{\Gamma}(T)\right] \rightarrow \pi_{0}(\boldsymbol{\Gamma}(T))
$$

shows that the middle term is Serre reflexive.

We deduce concrete consequences from this theorem, as in (4.2.1). Note that if $G$ is a connected proalgebraic group over $k$, then $\mathbf{E x t}_{k}^{n}(G, \mathbb{Z})=0$ for $n=0,1$ since $R \operatorname{Hom}_{k}(G, \mathbb{Z})=R \operatorname{Hom}_{k}(G, \mathbb{Q} / \mathbb{Z})[-1]$ by (2.3.3) (d) and $\operatorname{Hom}_{k}(G, \mathbb{Q} / \mathbb{Z})=0$ by (2.4.1) (a). This implies that

$$
\operatorname{Ext}_{k}^{n}(\boldsymbol{\Gamma}(T), \mathbb{Z})=\mathbf{E x t}_{k}^{n}\left(\pi_{0}(\boldsymbol{\Gamma}(T)), \mathbb{Z}\right)=\mathbf{E x t}_{k}^{n}\left(\pi_{0}\left(\mathcal{T}_{x}\right), \mathbb{Z}\right)
$$

for $n=0,1$. Hence the isomorphism $\theta_{T}$ in the above theorem induces, in degrees 0 and 1 , isomorphisms

$$
\begin{aligned}
\theta_{T}^{0}: \boldsymbol{\Gamma}\left(T^{\mathrm{CD}}\right) \stackrel{\sim}{\rightarrow} \operatorname{Hom}_{k}\left(\pi_{0}\left(\mathcal{T}_{x}\right), \mathbb{Z}\right), \\
\theta_{T}^{1}: \mathbf{H}^{1}\left(T^{\mathrm{CD}}\right) \stackrel{\sim}{\rightarrow} \operatorname{Ext}_{k}^{1}\left(\pi_{0}\left(\mathcal{T}_{x}\right), \mathbb{Z}\right)
\end{aligned}
$$

in FGEt/ $k$. We will put them together, and make explicit the isomorphism in degree 2 . We need notation to do this.

Let $I_{K}$ be the inertia group of the absolute Galois group of $K, X^{*}(T)=T^{\mathrm{CD}}$ the character lattice, $X_{*}(T)=\mathbf{H o m}_{K}\left(\mathbf{G}_{m}, T\right)$ the cocharacter lattice and $\hat{T}_{\text {tor }}=$ $X^{*}(T) \otimes_{\mathbb{Z}} \mathbb{Q} / \mathbb{Z}$ the torsion part of the dual torus 18 For an étale group $X$ over $K$, the $I_{K}$-coinvariants $X\left(K^{\text {sep }}\right) / I_{K}$ is a discrete $\mathrm{Gal}(\bar{k} / k)$-module. We denote the corresponding étale group over $k$ by $X / I_{K}$ by abuse of notation.

Proposition (8.3). From $H^{0}$ and $H^{1}$ of the isomorphism $\theta_{T}$, we can deduce an isomorphism

$$
\theta_{T}^{01}: \pi_{0}\left(\mathcal{T}_{x}\right) \stackrel{\sim}{\rightarrow} X_{*}(T) / I_{K}
$$

in FGEt/ $k$, and from $H^{2}$, an isomorphism

$$
\theta_{T}^{2}: \mathbf{H}^{1}\left(\hat{T}_{\text {tor }}\right) \stackrel{\sim}{\rightarrow} \operatorname{Ext}_{k}^{1}(\boldsymbol{\Gamma}(T), \mathbb{Q} / \mathbb{Z})
$$

in $\operatorname{IAlg}_{\mathrm{uc}} / k$.

We need a lemma.

Lemma (8.4). For any finitely generated étale group A over $K$, we have a natural isomorphism

$$
\tau_{\leq 1} R \boldsymbol{\Gamma} R \operatorname{Hom}_{K}(A, \mathbb{Z})=R \operatorname{Hom}_{k}\left(A / I_{K}, \mathbb{Z}\right)
$$

in $D(k)$, where $\tau$ denotes the truncation functor.

Proof. First note that $R \mathbf{H o m}_{K}$ between étale groups over $K$ is concentrated in degrees 0 and 1 since étale groups are locally just abelian groups and $\operatorname{Ext}_{\overline{\mathrm{Ab}}}^{\geq 2}=0$. The same is true for $R \mathbf{H o m}_{k}$. Let $0 \rightarrow B \rightarrow F \rightarrow A \rightarrow 0$ be an exact sequence with $F$ a finite free $\mathbb{Z}[G]$-module for some finite Galois extension $L / K$ and $G=$ $\operatorname{Gal}(L / K)$. Let $I_{G}$ be the inertia subgroup of $G$.

First we show that the distinguished triangle

$$
R \boldsymbol{\Gamma} R \operatorname{Hom}_{K}(A, \mathbb{Z}) \rightarrow R \boldsymbol{\Gamma} R \operatorname{Hom}_{K}(F, \mathbb{Z}) \rightarrow R \boldsymbol{\Gamma} R \operatorname{Hom}_{K}(B, \mathbb{Z})
$$

\footnotetext{
${ }^{18}$ Here we treat the torsion part of the dual torus algebraically without using $\mathbb{C}$ or $\overline{\mathbb{Q}}_{l}$. Therefore we ignore the Tate twist $\mathbb{Q} / \mathbb{Z}(1)$, which has nothing to do with the Galois action of $K$.
} 
induces a canonical morphism

$$
\left[\boldsymbol{\Gamma} \operatorname{Hom}_{K}(F, \mathbb{Z}) \rightarrow \boldsymbol{\Gamma} \operatorname{Hom}_{K}(B, \mathbb{Z})\right][-1] \rightarrow R \boldsymbol{\Gamma} R \operatorname{Hom}_{K}(A, \mathbb{Z}),
$$

where $[\cdot]$ denotes the mapping cone. Let $\mathbb{Z} \stackrel{\sim}{\rightarrow} J$ be an injective resolution in $\operatorname{Ab}\left(K_{\mathrm{fppf}} / k_{\mathrm{et}}^{\text {indrat }}\right)$. Then in the category of complexes in $\mathrm{Ab}\left(k_{\text {proet }}^{\text {indrat }}\right)$, we have natural morphism and isomorphism

$$
\begin{aligned}
& {\left[\boldsymbol{\Gamma}_{\operatorname{Hom}_{K}}(F, \mathbb{Z}) \rightarrow \boldsymbol{\Gamma} \operatorname{Hom}_{K}(B, \mathbb{Z})\right][-1]} \\
& \rightarrow\left[\tilde{\boldsymbol{\Gamma}} \operatorname{Hom}_{K}(F, J) \rightarrow \tilde{\boldsymbol{\Gamma}} \operatorname{Hom}_{K}(B, J)\right][-1] \\
& =\tilde{\boldsymbol{\Gamma}} \operatorname{Hom}_{K}([B \rightarrow F], J) .
\end{aligned}
$$

For another injective resolution $\mathbb{Z} \stackrel{\sim}{\rightarrow} J^{\prime}$ and a homotopy equivalence $J \stackrel{\sim}{\rightarrow} J^{\prime}$ over $\mathbb{Z}$, we have a commutative diagram

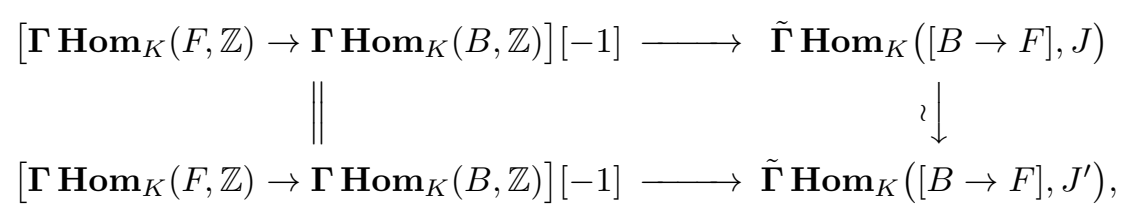

where the right vertical arrow is a homotopy equivalence. All homotopy equivalences $J \stackrel{\sim}{\rightarrow} J^{\prime}$ over $\mathbb{Z}$ are homotopic to each other, so they induce the same homotopy class for the right vertical arrow. We saw in the proof of (3.3.3) that the complex $\boldsymbol{\Gamma} \operatorname{Hom}_{K}([B \rightarrow F], J)$ represents $R \boldsymbol{\Gamma} R \operatorname{Hom}_{K}([B \rightarrow F], \mathbb{Z})$, or $R \boldsymbol{\Gamma} R \operatorname{Hom}_{K}(A, \mathbb{Z})$. Hence in $D\left(k_{\text {proet }}^{\text {indrat }}\right)$, we have a canonical morphism

$$
\left[\boldsymbol{\Gamma} \operatorname{Hom}_{K}(F, \mathbb{Z}) \rightarrow \boldsymbol{\Gamma} \operatorname{Hom}_{K}(B, \mathbb{Z})\right][-1] \rightarrow R \boldsymbol{\Gamma} R \operatorname{Hom}_{K}(A, \mathbb{Z}) .
$$

Since the left-hand side of this morphism is concentrated in degrees $\leq 1$, the morphism factors through the truncation $\tau_{\leq 1}$ of the right-hand side:

$$
\left[\boldsymbol{\Gamma} \operatorname{Hom}_{K}(F, \mathbb{Z}) \rightarrow \boldsymbol{\Gamma} \operatorname{Hom}_{K}(B, \mathbb{Z})\right][-1] \rightarrow \tau_{\leq 1} R \boldsymbol{\Gamma} R \operatorname{Hom}_{K}(A, \mathbb{Z}) .
$$

This is an isomorphism since $F$ is finite free over $\mathbb{Z}[G], \mathbf{H}^{1}(K, \mathbb{Z}[G])=\mathbf{H}^{1}(L, \mathbb{Z})=$ 0 and hence $R \boldsymbol{\Gamma} R \operatorname{Hom}_{K}(F, \mathbb{Z})=R \boldsymbol{\Gamma} \operatorname{Hom}_{K}(F, \mathbb{Z})$ has trivial $H^{1}$. The sheaf $\boldsymbol{\Gamma} \operatorname{Hom}_{K}(B, \mathbb{Z})$ is a lattice over $k$ by (3.4.3) (ㄷ). Its $\bar{k}$-points is $\operatorname{Hom}_{K^{\mathrm{ur}}}(B, \mathbb{Z})=$ $\operatorname{Hom}_{\bar{k}}\left(B / I_{G}, \mathbb{Z}\right)$ by (3.2.10). Therefore $\boldsymbol{\Gamma} \operatorname{Hom}_{K}(B, \mathbb{Z})=\operatorname{Hom}_{k}\left(B / I_{G}, \mathbb{Z}\right)$. Similarly we have $\boldsymbol{\Gamma} \operatorname{Hom}_{K}(F, \mathbb{Z})=\operatorname{Hom}_{k}\left(F / I_{G}, \mathbb{Z}\right)$. Therefore we have

$$
\begin{aligned}
& \tau_{\leq 1} R \boldsymbol{\Gamma} R \operatorname{Hom}_{K}(A, \mathbb{Z})=\left[\boldsymbol{\Gamma} \operatorname{Hom}_{K}(F, \mathbb{Z}) \rightarrow \boldsymbol{\Gamma} \operatorname{Hom}_{K}(B, \mathbb{Z})\right][-1] \\
& =\left[\operatorname{Hom}_{k}\left(F / I_{G}, \mathbb{Z}\right) \rightarrow \operatorname{Hom}_{k}\left(B / I_{G}, \mathbb{Z}\right)\right][-1] .
\end{aligned}
$$

Consider the following part of the long exact sequence of group homology:

$$
H_{1}\left(I_{G}, A\right) \rightarrow B / I_{G} \rightarrow F / I_{G} \rightarrow A / I_{G} \rightarrow 0 .
$$

This is an exact sequence of étale groups over $k$. Let $X$ be the kernel of $F / I_{G} \rightarrow$ $A / I_{G}$. In the short exact sequence $0 \rightarrow X \rightarrow F / I_{G} \rightarrow A / I_{G} \rightarrow 0$, the middle term $F / I_{G}$ is a lattice over $k$. Hence the same argument as above yields an isomorphism

$$
\left[\operatorname{Hom}_{k}\left(F / I_{G}, \mathbb{Z}\right) \rightarrow \operatorname{Hom}_{k}(X, \mathbb{Z})\right][-1]=R \operatorname{Hom}_{k}\left(A / I_{G}, \mathbb{Z}\right) .
$$

(No truncation $\tau_{\leq 1}$ is necessary on the right.) Consider the exact sequence

$$
0 \rightarrow \operatorname{Hom}_{k}(X, \mathbb{Z}) \rightarrow \operatorname{Hom}_{k}\left(B / I_{G}, \mathbb{Z}\right) \rightarrow \operatorname{Hom}_{k}\left(H_{1}\left(I_{G}, A\right), \mathbb{Z}\right)
$$


Since $G$ is finite, the group $H_{1}\left(I_{G}, A\right)$ is torsion ([Ser79, VIII, Cor. 1 to Prop. 4]), so the final term is zero. With $A / I_{G}=A / I_{K}$, we get

$$
\left[\operatorname{Hom}_{k}\left(F / I_{G}, \mathbb{Z}\right) \rightarrow \operatorname{Hom}_{k}\left(B / I_{G}, \mathbb{Z}\right)\right][-1]=R \operatorname{Hom}_{k}\left(A / I_{K}, \mathbb{Z}\right) .
$$

Combining all the above, we get the required isomorphism. This isomorphism is independent of the choice of the sequence $0 \rightarrow B \rightarrow F \rightarrow A \rightarrow 0$.

Proof of (8.3). We construct $\theta_{T}^{01}$. By (8.2), the natural morphism

$$
R \operatorname{Hom}_{k}\left(\pi_{0}(\boldsymbol{\Gamma}(T)), \mathbb{Z}\right) \rightarrow \tau_{\leq 1} R \operatorname{Hom}_{k}(\boldsymbol{\Gamma}(T), \mathbb{Z})
$$

is an isomorphism. Hence we have

$$
\tau_{\leq 1}\left(R \boldsymbol{\Gamma}(T)^{\mathrm{SD}}\right)=R \mathbf{H o m}_{k}\left(\pi_{0}(\boldsymbol{\Gamma}(T)), \mathbb{Z}\right)=R \operatorname{Hom}_{k}\left(\pi_{0}\left(\mathcal{T}_{x}\right), \mathbb{Z}\right) .
$$

Since $T^{\mathrm{CD}}=X^{*}(T)=\operatorname{Hom}_{K}\left(X_{*}(T), \mathbb{Z}\right)$, we have

$$
\tau_{\leq 1} R \boldsymbol{\Gamma}\left(T^{\mathrm{CD}}\right)=\tau_{\leq 1} R \boldsymbol{\Gamma} \operatorname{Hom}_{K}\left(X_{*}(T), \mathbb{Z}\right)=R \operatorname{Hom}_{k}\left(X_{*}(T) / I_{K}, \mathbb{Z}\right)
$$

using the lemma. Hence the isomorphism $\theta_{T}$ induces an isomorphism

$$
R \operatorname{Hom}_{k}\left(X_{*}(T) / I_{K}, \mathbb{Z}\right) \stackrel{\sim}{\rightarrow} R \operatorname{Hom}_{k}\left(\pi_{0}\left(\mathcal{T}_{x}\right), \mathbb{Z}\right)
$$

in $D^{b}(\mathrm{FGEt} / k)$. Apply SD to the both sides. Then (2.4.1) (b) gives an isomorphism $\theta_{T}^{01}: \pi_{0}\left(\mathcal{T}_{x}\right) \stackrel{\sim}{\rightarrow} X_{*}(T) / I_{K}$.

We construct $\theta_{T}^{2}$. Applying the direct limit by multiplication by $n \geq 1$ for $\theta_{T}$, we have

$$
\theta_{T} \otimes \mathbb{Q}: \underset{n}{\lim _{n}} R \boldsymbol{\Gamma}\left(T^{\mathrm{CD}}\right) \stackrel{\sim}{\rightarrow} \underset{n}{\lim } R \operatorname{Hom}_{k}(R \boldsymbol{\Gamma}(T), \mathbb{Z}) .
$$

For any $k^{\prime} \in k^{\text {indrat }}$, we have

$$
\underset{n}{\lim } R \Gamma\left(\mathbf{K}\left(k^{\prime}\right), T^{\mathrm{CD}}\right)=R \Gamma\left(\mathbf{K}\left(k^{\prime}\right), \underset{n}{\lim } T^{\mathrm{CD}}\right)=R \Gamma\left(\mathbf{K}\left(k^{\prime}\right), T^{\mathrm{CD}} \otimes_{\mathbb{Z}} \mathbb{Q}\right)
$$

by [Mil80, III, Rmk. 3.6], so $\lim _{n} R \boldsymbol{\Gamma}\left(T^{\mathrm{CD}}\right)=R \boldsymbol{\Gamma}\left(T^{\mathrm{CD}} \otimes_{\mathbb{Z}} \mathbb{Q}\right)$. Also

$$
\underset{n}{\lim _{n}} R \operatorname{Hom}_{k}\left(\boldsymbol{\Gamma}(T)_{0}, \mathbb{Z}\right)=R \operatorname{Hom}_{k}\left(\boldsymbol{\Gamma}(T)_{0}, \mathbb{Q}\right)=0
$$

by (2.3.1) (B)), (d) and (2.3.2) since $\boldsymbol{\Gamma}(T)_{0} \in \mathrm{PAlg} / k$, and

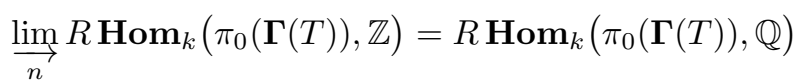

since $\pi_{0}(\boldsymbol{\Gamma}(T)) \in$ FGEt $/ k$ and $\operatorname{Ext}_{\mathrm{Ab}}^{n}(G, \cdot)$ for finitely generated $G$ commutes with filtered direct limits. Hence $\lim _{n} R \mathbf{H o m}_{k}(\boldsymbol{\Gamma}(T), \mathbb{Z})=R \operatorname{Hom}_{k}(\boldsymbol{\Gamma}(T), \mathbb{Q})$. Therefore

$$
\theta_{T} \otimes \mathbb{Q}: R \boldsymbol{\Gamma}\left(T^{\mathrm{CD}} \otimes_{\mathbb{Z}} \mathbb{Q}\right) \stackrel{\sim}{\rightarrow} R \operatorname{Hom}_{k}(R \boldsymbol{\Gamma}(T), \mathbb{Q}) .
$$

Together with $\theta_{T}$, we have a morphism

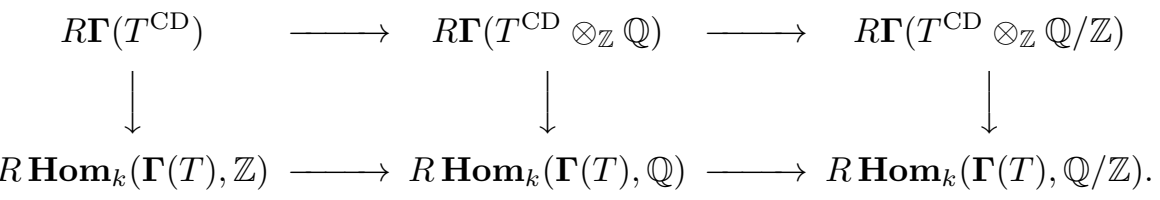

of distinguished triangles, whose left two vertical arrows are isomorphisms. Thus we get an isomorphism

$$
R \boldsymbol{\Gamma}\left(\hat{T}_{\text {tor }}\right) \stackrel{\sim}{\rightarrow} R \operatorname{Hom}_{k}(\boldsymbol{\Gamma}(T), \mathbb{Q} / \mathbb{Z}) .
$$

In degree 1 , it gives $\theta_{T}^{2}$. 
The existence of an isomorphism $\pi_{0}\left(\mathcal{T}_{x}\right) \cong X_{*}(T) / I_{K}$ was also obtained by Bertapelle and González-Avilés BGA15 in another method. Note that the result of Xarles XXar93] has been recovered in the course of the above proof.

We describe $\theta_{T}^{01}$. First we need a preparation about not necessarily totally ramified extensions (cf. [SY12, §4.2]). For a finite Galois extension $L / K$ with residue extension $k^{\prime} / k$, let $\mathbf{L}^{\times} \in \mathrm{Ab}\left(k_{\text {proet }}^{\text {indrat }}\right)$ be the sheaf defined in the same way as $\mathbf{K}^{\times}$:

$$
\mathbf{L}^{\times}\left(k^{\prime \prime}\right)=\left(\left(W\left(k^{\prime \prime}\right) \hat{\otimes}_{W\left(k^{\prime}\right)} \mathcal{O}_{L}\right) \otimes_{\mathcal{O}_{L}} L\right)^{\times}
$$

for $k^{\prime \prime} \in k^{\text {indrat }}$. On the other hand, we denote $\mathbf{L}_{k}^{\times}=\boldsymbol{\Gamma}\left(K, \operatorname{Res}_{L / K} \mathbf{G}_{m}\right)$, where $\operatorname{Res}_{L / K}$ is the Weil restriction. These sheaves are related in the following way: for any $k^{\prime \prime} \in k^{\text {indrat }}$, we have

$$
\mathbf{L}_{k}^{\times}\left(k^{\prime \prime}\right)=\left(\mathbf{K}\left(k^{\prime \prime}\right) \otimes_{K} L\right)^{\times}=\mathbf{L}^{\times}\left(k^{\prime \prime} \otimes_{k} k^{\prime}\right)
$$

(see [SY12, §4.2]; the notation there for $\mathbf{L}^{\times}$was $\mathbf{L}_{k^{\prime}}^{\times}$). Hence $\mathbf{L}_{k}^{\times}=\operatorname{Res}_{k^{\prime} / k} \mathbf{L}^{\times}$. Let $\operatorname{Res}_{k^{\prime} / k} \mathbb{Z}=\mathbb{Z}\left[\operatorname{Gal}\left(k^{\prime} / k\right)\right]$ be the group ring viewed as an étale group over $k$. Applying $\operatorname{Res}_{k^{\prime} / k}$ to the split exact sequence $0 \rightarrow \mathbf{U}_{L} \rightarrow \mathbf{L}^{\times} \rightarrow \mathbb{Z} \rightarrow 0$ in $\mathrm{Ab}\left(k_{\text {proet }}^{\text {indrat }}\right)$ (where $\mathbf{U}_{L}$ is defined similarly as $\mathbf{U}_{K}$ ), we have a split exact sequence

$$
0 \rightarrow \operatorname{Res}_{k^{\prime} / k} \mathbf{U}_{L} \rightarrow \mathbf{L}_{k}^{\times} \rightarrow \mathbb{Z}\left[\operatorname{Gal}\left(k^{\prime} / k\right)\right] \rightarrow 0
$$

in $\operatorname{Ab}\left(k_{\text {proet }}^{\text {indrat }}\right)$. The group $\operatorname{Res}_{k^{\prime} / k} \mathbf{U}_{L}$ is connected, so $\pi_{0}\left(\mathbf{L}_{k}^{\times}\right)=\mathbb{Z}\left[\operatorname{Gal}\left(k^{\prime} / k\right)\right]$.

Now we define a map that is going to be inverse to $\theta_{T}^{01}$. Let $\lambda: \mathbf{G}_{m} \rightarrow T$ be a morphism over $L$. The duality for the finite étale morphism $\operatorname{Spec} L \rightarrow \operatorname{Spec} K$ gives a morphism $\operatorname{Res}_{L / K} \mathbf{G}_{m} \rightarrow T$ over $K$. In other words, this is the composite of $\lambda: \operatorname{Res}_{L / K} \mathbf{G}_{m} \rightarrow \operatorname{Res}_{L / K} T$ with the norm map $\operatorname{Res}_{L / K} T \rightarrow T$ for $T$. Applying $\boldsymbol{\Gamma}$, we have a morphism $\mathbf{L}_{k}^{\times} \rightarrow \boldsymbol{\Gamma}(K, T)$ over $k$, Applying $\pi_{0}$, we have a morphism $\mathbb{Z}\left[\mathrm{Gal}\left(k^{\prime} / k\right)\right] \rightarrow \pi_{0}(\boldsymbol{\Gamma}(T))=\pi_{0}\left(\mathcal{T}_{x}\right)$ over $k$. The image of $1 \in$ $\mathbb{Z}\left[\operatorname{Gal}\left(k^{\prime} / k\right)\right]$ corresponds to a $k^{\prime}$-point of $\pi_{0}\left(\mathcal{T}_{x}\right)$. Thus we have a homomorphism $\operatorname{Hom}_{L}\left(\mathbf{G}_{m}, T\right) \rightarrow \Gamma\left(k^{\prime}, \pi_{0}\left(\mathcal{T}_{x}\right)\right)$. This is compatible with the action of $\operatorname{Gal}(L / K)$ (which factors through $\operatorname{Gal}\left(k^{\prime} / k\right)$ on the right). It is also compatible with extending $L$. Therefore we obtain a homomorphism $X_{*}(T) / I_{K} \rightarrow \pi_{0}\left(\mathcal{T}_{x}\right)$ of étale groups over $k$, which we denote by $\varphi$.

Proposition (8.6). The homomorphism $\varphi: X_{*}(T) / I_{K} \rightarrow \pi_{0}\left(\mathcal{T}_{x}\right)$ just defined is the inverse map of the isomorphism $\theta_{T}^{01}$.

Proof. We may assume that $k$ is algebraically closed, so $G_{K}:=I_{K}$ is the absolute Galois group of $K$. It is enough to compare the homomorphisms induced on the $\mathbb{Q} / \mathbb{Z}$-duals $\operatorname{Hom}_{\mathrm{Ab}}\left(X_{*}(T) / I_{K}, \mathbb{Q} / \mathbb{Z}\right)$ and $\operatorname{Hom}_{\mathrm{Ab}}\left(\pi_{0}\left(\mathcal{T}_{x}\right), \mathbb{Q} / \mathbb{Z}\right)$.

By (8.5), we have

$$
R \Gamma\left(\hat{T}_{\text {tor }}\right) \stackrel{\sim}{\rightarrow} R \operatorname{Hom}_{k}(\boldsymbol{\Gamma}(T), \mathbb{Q} / \mathbb{Z})
$$

Therefore

$$
\Gamma\left(\hat{T}_{\text {tor }}\right) \stackrel{\sim}{\rightarrow} \operatorname{Hom}_{k}(\boldsymbol{\Gamma}(T), \mathbb{Q} / \mathbb{Z}) \stackrel{\sim}{\rightarrow} \operatorname{Hom}_{\mathrm{Ab}}\left(\pi_{0}(\boldsymbol{\Gamma}(T)), \mathbb{Q} / \mathbb{Z}\right) .
$$

Also, since $\hat{T}_{\text {tor }}=X^{*}(T) \otimes_{\mathbb{Z}} \mathbb{Q} / \mathbb{Z}=\operatorname{Hom}_{K}\left(X_{*}(T), \mathbb{Q} / \mathbb{Z}\right)$, we have a natural isomorphism

$$
\begin{aligned}
\Gamma\left(\hat{T}_{\text {tor }}\right) & \stackrel{\sim}{\rightarrow} \operatorname{Hom}_{K}\left(X_{*}(T), \mathbb{Q} / \mathbb{Z}\right) \\
& \stackrel{\sim}{\rightarrow} \operatorname{Hom}_{\mathrm{Ab}}\left(X_{*}(T) / G_{K}, \mathbb{Q} / \mathbb{Z}\right) .
\end{aligned}
$$


By construction, the $\mathbb{Q} / \mathbb{Z}$-dual of $\theta_{T}^{01}$ is given by the composite

$$
\operatorname{Hom}_{\mathrm{Ab}}\left(X_{*}(T) / G_{K}, \mathbb{Q} / \mathbb{Z}\right) \stackrel{\sim}{\leftarrow}\left(\hat{T}_{\text {tor }}\right) \stackrel{\sim}{\rightarrow} \operatorname{Hom}_{\mathrm{Ab}}\left(\pi_{0}(\boldsymbol{\Gamma}(T)), \mathbb{Q} / \mathbb{Z}\right) .
$$

It is enough to show that the morphism

$$
\Gamma\left(\hat{T}_{\text {tor }}\right) \stackrel{\sqrt[8.7]{\sim}}{\rightarrow} \operatorname{Hom}_{\mathrm{Ab}}\left(\pi_{0}(\boldsymbol{\Gamma}(T)), \mathbb{Q} / \mathbb{Z}\right) \stackrel{\varphi}{\rightarrow} \operatorname{Hom}_{\mathrm{Ab}}\left(X_{*}(T) / G_{K}, \mathbb{Q} / \mathbb{Z}\right)
$$

coincides with (8.8).

Let $\chi \otimes r \in \hat{T}_{\text {tor }}=X^{*}(T) \otimes \mathbb{Q} / \mathbb{Z}$ be $G_{K}$-invariant, where $\chi: T \rightarrow \mathbf{G}_{m}$ is a morphism over a finite Galois extension $L / K$ and $r \in \mathbb{Q} / \mathbb{Z}$. Then we have a morphism

$$
\boldsymbol{\Gamma}(L, T) \stackrel{\chi_{\chi}}{\rightarrow} \mathbf{L}^{\times} \stackrel{v_{L}}{\rightarrow} \mathbb{Z} \stackrel{r}{\rightarrow} \mathbb{Q} / \mathbb{Z}
$$

over $k$. The norm map $N: \boldsymbol{\Gamma}(L, T) \rightarrow \boldsymbol{\Gamma}(K, T)$ identifies $\boldsymbol{\Gamma}(K, T)$ as the $G_{K^{-}}$ coinvariants of $\boldsymbol{\Gamma}(L, T)$ as we saw in the second paragraph of the proof of (8.1). The $G_{K}$-invariance of $\chi \otimes r$ implies that the above $r v_{L} \circ \chi$ factors through $\boldsymbol{\Gamma}(T)=$ $\boldsymbol{\Gamma}(K, T)$ and hence through its $\pi_{0}$. This defines a homomorphism

$$
r v_{L} \circ \chi \circ N^{-1}: \pi_{0}\left(\mathcal{T}_{x}\right) \rightarrow \mathbb{Q} / \mathbb{Z}
$$

This is the element given by (8.7).

We calculate the image (or pullback) of this element by $\varphi$. If $\lambda: \mathbf{G}_{m} \rightarrow T$ is a morphism over $L$, then $\varphi(\lambda)=N \lambda\left(\pi_{L}\right)$ by the construction of $\varphi$, where $\pi_{L}$ is a prime element of $L$. Hence the pullback by $\varphi$ of $r v_{L} \circ \chi \circ N^{-1}$ sends $\lambda$ to

$$
\begin{aligned}
r v_{L} \circ \chi \circ N^{-1} \circ N \circ \lambda\left(\pi_{L}\right) & =r v_{L} \circ \chi \circ \lambda\left(\pi_{L}\right) \\
& =r v_{L}\left(\pi_{L}^{\langle\chi, \lambda\rangle}\right) \\
& =r\langle\chi, \lambda\rangle \in \mathbb{Q} / \mathbb{Z},
\end{aligned}
$$

where $\langle\chi, \lambda\rangle \in \mathbb{Z}$ is the natural pairing between a character and a cocharacter. The obtained assignment $\lambda \mapsto r\langle\chi, \lambda\rangle$ agrees with the image of $\chi \otimes r$ under the homomorphism (8.8).

We describe $\theta_{T}^{2}$. One method to do this is to go back to the proof of (8.1), namely use Galois descent to reduce to Serre's local class field theory; cf. (6.13) (b). Another method is to use the sequence $0 \rightarrow T[n] \rightarrow T \stackrel{n}{\rightarrow} T \rightarrow 0$ for each $n \geq 1$ to reduce it to the description of the duality with coefficients in finite flat group schemes $T[n]$. We give here yet another, more direct method. It is enough to describe the morphism induced on $k$-points since the groups involved with $\theta_{T}^{2}$ are ind-algebraic and $k$ can be any perfect field.

An element of

$$
H^{1}\left(\hat{T}_{\text {tor }}\right)=H^{2}\left(T^{\mathrm{CD}}\right)=\operatorname{Ext}_{K}^{2}\left(\mathbb{Z}, T^{\mathrm{CD}}\right)
$$

corresponds to an extension class

$$
E: 0 \rightarrow T^{\mathrm{CD}} \rightarrow X_{2} \rightarrow X_{1} \rightarrow \mathbb{Z} \rightarrow 0 .
$$

The terms $X_{i}$ can be chosen more explicitly as follow. If the element lives in $H^{2}\left(G, \Gamma\left(L, T^{\mathrm{CD}}\right)\right)$ with $G=\operatorname{Gal}(L / K)$ for a finite Galois extension $L / K$, then it gives a morphism of complexes of $G$-modules from the standard resolution of $\mathbb{Z}$ to $\Gamma\left(L, T^{\mathrm{CD}}\right)[2]$. By pushing out the standard resolution

$$
\cdots \rightarrow \mathbb{Z}\left[G^{4}\right] \rightarrow \mathbb{Z}\left[G^{3}\right] \rightarrow \mathbb{Z}\left[G^{2}\right] \rightarrow \mathbb{Z}[G] \rightarrow \mathbb{Z} \rightarrow 0
$$


(of homogeneous chains) at the degree -2 term $\mathbb{Z}\left[G^{3}\right]$ to $\Gamma\left(L, T^{\mathrm{CD}}\right)$, we get a choice of (the $L$-valued points of) the extension class $E$ :

$$
0 \rightarrow \Gamma\left(L, T^{\mathrm{CD}}\right) \rightarrow X \rightarrow \mathbb{Z}[G] \rightarrow \mathbb{Z} \rightarrow 0 .
$$

All these terms are lattices over $K$. The image of $E$ in $\operatorname{Ext}_{K}^{2}\left(T, \mathbf{G}_{m}\right)$ is given by taking its Cartier dual

$$
0 \rightarrow \mathbf{G}_{m} \rightarrow \operatorname{Res}_{L / K} \mathbf{G}_{m} \rightarrow X^{\mathrm{CD}} \rightarrow T \rightarrow 0 .
$$

Apply $R \tilde{\boldsymbol{\Gamma}}$. Since the terms are all tori, we have an exact sequence

$$
0 \rightarrow \mathbf{K}^{\times} \rightarrow \mathbf{L}_{k}^{\times} \rightarrow \boldsymbol{\Gamma}\left(X^{\mathrm{CD}}\right) \rightarrow \boldsymbol{\Gamma}(T) \rightarrow 0
$$

in $\mathrm{Ab}\left(k_{\text {proet }}^{\text {indrat }}\right)$ by (3.4.3). By pushing it out by the valuation map $\mathbf{K}^{\times} \rightarrow \mathbb{Z}$, we get an extension

$$
0 \rightarrow \mathbb{Z} \rightarrow \mathbf{L}_{k}^{\times} / \mathbf{U}_{K} \rightarrow \boldsymbol{\Gamma}\left(X^{\mathrm{CD}}\right) \rightarrow \boldsymbol{\Gamma}(T) \rightarrow 0 .
$$

This is the element of $\operatorname{Ext}_{k}^{2}(\boldsymbol{\Gamma}(T), \mathbb{Z})$ corresponding to $\theta_{T}$. Let $\psi$ be the middle morphism $\mathbf{L}_{k}^{\times} \rightarrow \boldsymbol{\Gamma}\left(X^{\mathrm{CD}}\right)$. Recall the morphism $\mathbf{L}_{k}^{\times} \rightarrow \mathbb{Z}\left[\mathrm{Gal}\left(k^{\prime} / k\right)\right]$ defined before (8.6). The maximal constant quotient of the étale group $\mathbb{Z}\left[\mathrm{Gal}\left(k^{\prime} / k\right)\right]$ is $\mathbb{Z}$. Let $\mathbf{U}_{L, k^{\prime} / k}$ be the kernel of the composite $\mathbf{L}_{k}^{\times} \rightarrow \mathbb{Z}\left[\operatorname{Gal}\left(k^{\prime} / k\right)\right] \rightarrow \mathbb{Z}$. Let $n=[L: K]$. Then the above extension class comes from the extension

$$
0 \rightarrow \mathbb{Z} / n \mathbb{Z} \rightarrow \boldsymbol{\Gamma}\left(X^{\mathrm{CD}}\right) / \psi\left(\mathbf{U}_{L, k^{\prime} / k}\right) \rightarrow \boldsymbol{\Gamma}(T) \rightarrow 0
$$

in $\operatorname{Ext}_{k}^{1}(\boldsymbol{\Gamma}(T), \mathbb{Z} / n \mathbb{Z})$. By pushing it out by $\mathbb{Z} / n \mathbb{Z} \hookrightarrow \mathbb{Q} / \mathbb{Z}$, we get an corresponding extension class in $\operatorname{Ext}_{k}^{1}(\boldsymbol{\Gamma}(T), \mathbb{Q} / \mathbb{Z})$. This is the element given by $\theta_{T}^{2}$.

Remark (8.9). As in (7.3), we can give a statement on $R \Gamma(T)$ and $R \Gamma\left(T^{\mathrm{CD}}\right)$ even if $k$ is not algebraically closed, thanks to the P-acyclicity of $R \boldsymbol{\Gamma}(T)$ and $R \boldsymbol{\Gamma}\left(T^{\mathrm{CD}}\right)$. Let $\left(\mathcal{T}_{x}\right)_{\mathrm{t}}$ be the maximal torus of the special fiber $\mathcal{T}_{x}$ and set $Q_{k, T}=$ $R \lim _{n} R \Gamma\left(k_{\text {et }},\left(\mathcal{T}_{x}\right)_{\mathrm{t}}\right)$. Then by the same reasoning as the cited remark, we have

$$
\begin{gathered}
R \Gamma\left(T^{\mathrm{CD}}\right)=R \operatorname{Hom}_{k_{\mathrm{et}}^{\text {indrat }}}(\boldsymbol{\Gamma}(T), \mathbb{Z}), \\
{\left[Q_{k, T} \rightarrow R \Gamma(T)\right]=R \operatorname{Hom}_{k_{\mathrm{et}}^{\text {indrat }}}\left(R \boldsymbol{\Gamma}\left(T^{\mathrm{CD}}\right), \mathbb{Z}\right)}
\end{gathered}
$$

in $D(\mathrm{Ab})$. By the isomorphism (8.5), we have

$$
R \Gamma\left(\hat{T}_{\text {tor }}\right)=R \operatorname{Hom}_{k_{\mathrm{et}}^{\text {indrat }}}(\boldsymbol{\Gamma}(T), \mathbb{Q} / \mathbb{Z}) .
$$

If we look at the maximal subgroups of $\boldsymbol{\Gamma}(T)$ and $\boldsymbol{\Gamma}\left(T^{\mathrm{CD}}\right)$ whose quotients are lattices, then the corresponding parts of the right-hand sides of these isomorphisms may be written by $R \operatorname{Hom}_{\mathrm{PAlg} / k}$ and $R \operatorname{Hom}_{\mathrm{IAlg} / k}$.

\section{Duality with Coefficients in 1-motives}

Next we treat the case of coefficients in 1-motives. We only formulate and prove the duality, without giving its full description. Let $M=[Y \rightarrow G]$ be a 1-motive over $K$ in the sense of Deligne, where $Y$ is a lattice placed in degree -1 and $G$ a semi-abelian variety. We can naturally regard $M$ as an object in $D\left(K_{\mathrm{fppf}}\right)$ and hence in $D\left(K_{\mathrm{fppf}} / k_{\mathrm{et}}^{\text {indrat }}\right)$. We calculate $R \boldsymbol{\Gamma}(M)$ by (3.4.3). Applying $R \boldsymbol{\Gamma}$ on $M$, we have a long exact sequence

$$
\begin{aligned}
& 0 \rightarrow \mathbf{H}^{-1}(M) \rightarrow \boldsymbol{\Gamma}(Y) \rightarrow \boldsymbol{\Gamma}(G) \rightarrow \mathbf{H}^{0}(M) \\
& \rightarrow \mathbf{H}^{1}(Y) \rightarrow \mathbf{H}^{1}(G) \rightarrow \mathbf{H}^{1}(M) \rightarrow \mathbf{H}^{2}(Y) \rightarrow 0
\end{aligned}
$$


in $\mathrm{Ab}\left(k_{\mathrm{et}}^{\text {indrat }}\right)$ and $\mathbf{H}^{n}(M)=0$ for $n \neq-1,0,1$. We know that $\boldsymbol{\Gamma}(Y)$ is a lattice over $k, \boldsymbol{\Gamma}(G)$ is an extension of a lattice by a $\mathrm{P}$-acyclic proalgebraic group (which can be checked by reducing it to the case of an abelian variety and a torus, using the fact that an extension of a proalgebraic group by $\mathbb{Q}$ splits by (2.3.3) $), \mathbf{H}^{1}(Y) \in \mathrm{FEt} / k$, and $\mathbf{H}^{1}(G), \mathbf{H}^{2}(Y) \in \operatorname{IAlg}_{\mathrm{uc}} / k$. Hence $\mathbf{H}^{-1}(M)$ is a lattice over $k$ and $\mathbf{H}^{1}(M) \in$ $\mathrm{IAlg}_{\mathrm{uc}} / k$. Since all the terms but $\mathbf{H}^{0}(M)$ are $\mathrm{P}$-acyclic, we know that $\mathbf{H}^{0}(M)$ is P-acyclic, Therefore $R \boldsymbol{\Gamma}(M)$ is P-acyclic, and we will write $R \tilde{\boldsymbol{\Gamma}}(M)=R \boldsymbol{\Gamma}(M) \in$ $D\left(k_{\text {proet }}^{\text {indrat }}\right)$.

Let $M^{\vee}$ be the dual 1-motive of $M$. In $D\left(K_{\mathrm{fppf}}\right)$, the complex $M^{\vee}$ is identified with $\tau_{\leq 0} R \mathbf{H o m}_{K}\left(M, \mathbf{G}_{m}[1]\right)$. Note the shift: if $M$ is a torus $T$, then $M^{\vee}$ is the character lattice $T^{\mathrm{CD}}$ placed in degree -1 . We have a morphism

$$
\begin{aligned}
R \boldsymbol{\Gamma}\left(M^{\vee}\right) & \rightarrow R \tilde{\mathbf{\Gamma}} R \mathbf{H o m}_{K}\left(M, \mathbf{G}_{m}[1]\right) \\
& \rightarrow R \mathbf{H o m}_{k}(R \boldsymbol{\Gamma}(M), \mathbb{Z})[1]=R \boldsymbol{\Gamma}(M)^{\mathrm{SD}}[1]
\end{aligned}
$$

in $D\left(k_{\text {proet }}^{\text {indrat }}\right)$ and hence a morphism

$$
R \boldsymbol{\Gamma}\left(M^{\vee}\right)^{\mathrm{SDSD}} \rightarrow R \boldsymbol{\Gamma}(M)^{\mathrm{SD}}[1]
$$

by taking SD of the both sides and replacing $M$ with $M^{\vee}$.

Theorem (9.1). The above defined morphism

$$
R \boldsymbol{\Gamma}\left(M^{\vee}\right)^{\mathrm{SDSD}} \rightarrow R \boldsymbol{\Gamma}(M)^{\mathrm{SD}}[1]
$$

in $D\left(k_{\text {proet }}^{\text {indrat }}\right)$ is an isomorphism.

Proof. Note that the both sides are triangulated functors on $M$. Therefore if $M_{1} \rightarrow$ $M_{2} \rightarrow M_{3}$ is a distinguished triangle of 1-motives and the theorem is true for any two of them, then so is the third. Let $M=[Y \rightarrow G]$ with $Y$ a lattice over $K, G$ semi-abelian with torus part $T$ and $A=G / T$. We have the weight filtration $W_{n} M$ of $M$, where $W_{0} M=M, W_{-1} M=[0 \rightarrow G], W_{-2} M=[0 \rightarrow T]$ and $W_{-3} M=0$. The distinguished triangles

$$
W_{-1} M \rightarrow M \rightarrow[Y \rightarrow 0], \quad W_{-2} M \rightarrow W_{-1} M \rightarrow[0 \rightarrow A]
$$

show that the statement for $M$ is reduced to that of $A, T$ and $Y$. These are (4.1.2) and (8.1).

Remark (9.2). In the proof of (4.1.2), we have used the case of semistable abelian varieties. The above formulation for 1-motives allows us to deduce this case from the case of good reduction abelian varieties, as follows.

Let $A$ be a semistable abelian variety over $K$. Then by rigid analytic uniformization, we have an exact sequence $0 \rightarrow Y \rightarrow G \rightarrow A \rightarrow 0$ of rigid $K$-groups, where $Y$ is a lattice with unramified Galois action and $G$ an extension of a good reduction abelian variety by an unramified torus. Set $M=[Y \rightarrow G]$. Let $K_{\mathrm{et}}^{\mathrm{fp}} / k^{\text {indrat }}$ be the modification of the category $K_{\text {et }} / k^{\text {indrat }}$ where objects are pairs $\left(S, k_{S}\right)$ with $k_{S} \in k^{\text {indrat }}$ and $S$ an étale $\mathbf{K}^{\mathrm{fp}}\left(k_{S}\right)$-algebra. Denote $\hat{S}=S \otimes_{\mathbf{K}^{\mathrm{fp}}\left(k_{S}\right)} \mathbf{K}\left(k_{S}\right)$. Let Spec $K_{\mathrm{et}}^{\mathrm{fp}} / k_{\mathrm{et}}^{\text {indrat }}$ be the étale site on $K_{\mathrm{et}}^{\mathrm{fp}} / k^{\text {indrat }}$. The functors $k^{\prime} \mapsto\left(\mathbf{K}^{\mathrm{fp}}\left(k^{\prime}\right), k^{\prime}\right)$ and $\left(S, k_{S}\right) \mapsto\left(\hat{S}, k_{S}\right)$ define morphisms

$$
\text { Spec } K_{\text {et }} / k_{\text {et }}^{\text {indrat }} \stackrel{f}{\rightarrow} \operatorname{Spec} K_{\mathrm{et}}^{\mathrm{fp}} / k_{\mathrm{et}}^{\text {indrat }} \stackrel{\pi^{\mathrm{fp}}}{\rightarrow} \operatorname{Spec} k_{\mathrm{et}}^{\text {indrat }}
$$

of sites. The pushforward $f_{*}$ is exact by [Suz13, Lem. 2.5.5]. Note that the ring $\hat{S}$ for any étale $\mathbf{K}^{\mathrm{fp}}\left(k_{S}\right)$-algebra $S$ is a complete topological $K$-algebra. We can 
show that the sequence $0 \rightarrow Y \rightarrow G \rightarrow A \rightarrow 0$ of rigid $K$-groups induces an exact sequence $0 \rightarrow f_{*} Y \rightarrow f_{*} G \rightarrow f_{*} A \rightarrow 0$ in $\mathrm{Ab}\left(K_{\mathrm{et}}^{\mathrm{fp}} / k_{\mathrm{et}}^{\text {indrat }}\right)$, by a rigid analytic version of the approximation argument used in the proof of the cited lemma. Hence we have $f_{*} M \stackrel{\sim}{\rightarrow} f_{*} A$ in $\operatorname{Ab}\left(K_{\mathrm{et}}^{\mathrm{fp}} / k_{\mathrm{et}}^{\text {indrat }}\right)$. Applying $R \pi_{*}^{\mathrm{fp}}$ and using the exactness of $f_{*}$, we have an isomorphism

$$
R \boldsymbol{\Gamma}(M) \stackrel{\sim}{\rightarrow} R \boldsymbol{\Gamma}(A) .
$$

Hence (4.1.2) for $A$ is reduced to (9.1) for $M$. The cases of $\mathbf{G}_{m}$ and $\mathbb{Z}$ were explained in (8.1), which was essentially Serre's local class field theory. Hence we are reduced to the case of abelian varieties with good reduction.

Note that the case of abelian varieties with good reduction is further reduced to the case of finite flat group schemes over $\mathcal{O}_{K}$ obtained as the torsion part, as explained in Bégueri [Bég81, §7.1] and Bester [Bes78, §2.7]. With this argument, we can avoid Bertapelle's and Werner's results, and prove Grothendieck's and S̆afarevič's conjectures directly from the Bégueri-Bester duality for $\mathcal{O}_{K}$ with coefficients in finite flat group schemes.

\section{Connection With Classical STATEMENTS For Finite RESidue Fields}

We show that the duality theorems in this paper induce classical duality statements when the residue field $k$ is finite. Basically this can be done as follows. Take the derived global section $R \Gamma\left(k_{\text {proet }}, \cdot\right)$ of the both sides of our duality to translate $R \boldsymbol{\Gamma}(K, A) \in D\left(k_{\text {proet }}^{\text {indrat }}\right)$ into $R \Gamma(K, A) \in D(\mathrm{Ab})$. Then use Lang's theorem (see below)

$$
\operatorname{Ext}_{k}^{1}(C, \mathbb{Q} / \mathbb{Z})=\operatorname{Hom}_{\mathrm{Ab}}(C(k), \mathbb{Q} / \mathbb{Z})
$$

for a connected quasi-algebraic group $C$ over $k$ to translated the Serre dual to the Pontryagin dual. We can actually treat not only Galois group cohomology but also Weil group cohomology, and thanks to our setting of the pro-étale topology, recover classical dualities that take profinite group structures on cohomology groups into account.

We need to define and study several notions for this. Assume that $k=\mathbb{F}_{q}$. Let $\bar{k}$ be an algebraic closure of $k$. We denote by $F$ the $q$-th power Frobenius homomorphism on any $k$-algebra. It induces an action on any object of $\mathrm{Ab}\left(k_{\text {proet }}^{\text {indrat }}\right)$. For $A \in D\left(k_{\text {proet }}^{\text {indrat }}\right)$, we denote the mapping cone of $F-1: A \rightarrow A$ by $[A \stackrel{F-1}{\rightarrow} A]$. With a shift, we have a triangulated functor

$$
D\left(k_{\text {proet }}^{\text {indrat }}\right) \rightarrow D\left(k_{\text {proet }}^{\text {indrat }}\right), \quad A \mapsto[A \rightarrow A][-1] .
$$

This is the mapping fiber of $F-1: A \rightarrow A$.

Next, as in 2.1, let $k_{\text {proet }}$ be the category of ind-étale $k$-algebras and Spec $k_{\text {proet }}$ the pro-étale site on $k_{\text {proet }}$. Let $k_{\text {prozar }}$ be the full subcategory of $k_{\text {proet }}$ consisting of filtered unions $\bigcup k_{\lambda}^{\prime}$ where each $k_{\lambda}^{\prime}$ is a finite product of copies of $k$. For $k^{\prime} \in k_{\text {prozar }}$, we say that a finite family $\left\{k_{i}^{\prime}\right\}$ of objects of $k_{\text {prozar }}$ over $k^{\prime}$ is a covering if $k^{\prime} \rightarrow \prod_{i} k_{i}^{\prime}$ is faithfully flat. This defines a site, which we call the pro-Zariski site of $k$ and denote by Spec $k_{\text {prozar. }}$ This is equivalent to the pro-étale site $\operatorname{Spec} \bar{k}_{\text {proet }}$ of $\bar{k}$ via the functor $k^{\prime} \in k_{\text {prozar }} \mapsto k^{\prime} \otimes_{k} \bar{k} \in \bar{k}_{\text {proet. }}$. It is also equivalent to the site of profinite sets given in [BS15, Ex. 4.1.10]. By composing the continuous map of sites Spec $k_{\text {proet }}^{\text {indrat }} \rightarrow$ Spec $\bar{k}_{\text {proet }}$ defined by the identity with the identification 
Spec $\bar{k}_{\text {proet }} \cong$ Spec $k_{\text {prozar }}$, we have a continuous map of sites

$$
f: \text { Spec } k_{\text {proet }}^{\text {indrat }} \rightarrow \text { Spec } k_{\text {prozar }},
$$

which is defined by the functor $k_{\text {prozar }} \rightarrow k_{\text {proet }}$ given by $k^{\prime} \mapsto k^{\prime} \otimes_{k} \bar{k}$. Its pushforward functor $f_{*}: \mathrm{Ab}\left(k_{\text {proet }}^{\text {indrat }}\right) \rightarrow \mathrm{Ab}\left(k_{\text {prozar }}\right)$ is exact, which induces a triangulated functor

$$
f_{*}: D\left(k_{\text {proet }}^{\text {indrat }}\right) \rightarrow D\left(k_{\text {prozar }}\right) .
$$

The functor $f_{*}$ is a sort of a geometric points functor in the following sense. For any $A \in \mathrm{Ab}\left(k_{\text {proet }}^{\text {indrat }}\right)$ we have $\left(f_{*} A\right)(k)=A(\bar{k})$. If $A$ is locally of finite presentation, we may view the abelian group $A(\bar{k})$ as a constant sheaf on Spec $k_{\text {prozar }}$, and for any $k^{\prime}=\bigcup k_{\lambda}^{\prime} \in k_{\text {prozar }}$ with $k_{\lambda}$ a finite product of copies of $k$, we have

$$
\left(f_{*} A\right)\left(k^{\prime}\right)=\lim _{\longrightarrow} A\left(k_{\lambda}^{\prime} \otimes_{k} \bar{k}\right)=\lim _{\longrightarrow} A(\bar{k})\left(k_{\lambda}^{\prime}\right)=A(\bar{k})\left(k^{\prime}\right) .
$$

Thus $f_{*} A=A(\bar{k})$ in $\mathrm{Ab}\left(k_{\text {prozar }}\right)$ if $A$ is locally of finite presentation. Note that $f_{*}$ commutes with filtered direct limits and arbitrary inverse limits. Hence if $A=$ $\lim _{\lambda} \lim _{\lambda^{\prime}} A_{\lambda \lambda^{\prime}} \in \mathrm{IPAlg} / k$ with $A_{\lambda \lambda^{\prime}} \in \mathrm{Alg} / k$, then $f_{*} A=\lim _{\lambda} \lim _{\lambda^{\prime}}\left(A_{\lambda \lambda^{\prime}}(\bar{k})\right)$ in $\overrightarrow{\mathrm{Ab}}\left(k_{\text {prozar }}\right)$.

Now we define a functor $R \Gamma\left(k_{W}, \cdot\right)$ to be the composite of (10.1) and (10.2):

$$
\begin{gathered}
R \Gamma\left(k_{W}, \cdot\right): D\left(k_{\text {proet }}^{\text {indrat }}\right) \rightarrow D\left(k_{\text {prozar }}\right), \\
R \Gamma\left(k_{W}, A\right)=f_{*}[A \stackrel{F-1}{\rightarrow} A] .
\end{gathered}
$$

For $A \in \mathrm{Ab}\left(k_{\text {proet }}^{\text {indrat }}\right)$, we define $H^{n}\left(k_{W}, A\right) \in \mathrm{Ab}\left(k_{\text {prozar }}\right)$ to be the $n$-th cohomology of $R \Gamma\left(k_{W}, A\right)$, and set $\Gamma\left(k_{W}, A\right)=H^{0}\left(k_{W}, A\right)$. Obviously $H^{n}\left(k_{W}, A\right)=0$ for $n \neq 0,1$. We have an exact sequence

$$
0 \rightarrow \Gamma\left(k_{W}, A\right)(k) \rightarrow A(\bar{k}) \stackrel{F-1}{\rightarrow} A(\bar{k}) \rightarrow H^{1}\left(k_{W}, A\right)(k) \rightarrow 0,
$$

so that the global section of $R \Gamma\left(k_{W}, A\right) \in D\left(k_{\text {prozar }}\right)$ is $R \Gamma\left(W_{k}, A(\bar{k})\right) \in D(\mathrm{Ab})$, where $W_{k} \cong \mathbb{Z}$ is the Weil group of $k$. Thus $R \Gamma\left(k_{W}, \cdot\right)$ is an enhancement of the Weil group cohomology of $k$ as a functor valued in $D\left(k_{\text {prozar }}\right)$.

Let Fin be the category of finite abelian groups. Let PFin, IFin, IPFin its procategory, indcategory, ind-procategory, respectively. Note that IFin is equivalent to the category of torsion abelian groups. A finite set $X$ can be identified with a finite $k$-scheme $\bigsqcup_{x \in X}$ Spec $k$ and hence a finite product $\prod_{x \in X} k$ of copies of $k$. This extends to a functor from the category of profinite sets to $k_{\text {prozar }} \subset k^{\text {indrat }}$. Hence we have an additive functor IPFin $\rightarrow \mathrm{Ab}\left(k_{\text {prozar }}\right) \subset \mathrm{Ab}\left(k_{\text {proet }}^{\text {indrat }}\right)$. There is also an obvious functor $\mathrm{Ab} \rightarrow \mathrm{Ab}\left(k_{\text {prozar }}\right)$ that sends an abelian group $A$ to $\bigsqcup_{a \in A} \operatorname{Spec} k$.

\section{Proposition (10.3).}

(a) We have fully faithful embeddings

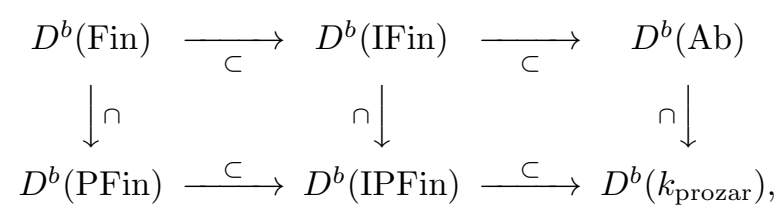

of triangulated categories. 
(b) If $A \in \mathrm{Alg} / k, \mathrm{PAlg} / k, \mathrm{IAlg} / k$ or $\mathrm{IPAlg} / k$, then the kernel and cokernel of $F-1: A \rightarrow A$ are in Fin, PFin, IFin or IPFin, respectively. We have $H^{n}\left(k_{W}, A\right) \in$ Fin, PFin, IFin or IPFin, respectively, for any $n$.

Proof. (国) For any $B \in \mathrm{Ab}$ and $n \geq 1$, we have $\operatorname{Ext}_{k_{\text {prozar }}}^{n}(\mathbb{Z}, B)=H^{n}\left(k_{\text {prozar }}, B\right)=$ 0 by the same proof as [Suz13, Prop. 3.3.1] (Zariski cohomology of a field is trivial). It follows that $R \operatorname{Hom}_{k_{\text {prozar }}}(A, B)=R \operatorname{Hom}_{\text {Fin }}(A, B)$ if $A, B \in$ Fin. From this, we can deduce the rest of the statements in the same way as we did in the proof of (2.3.4) (which ultimately will need the cubical construction for the limit arguments Suz13, Lem. 3.8.2]).

Alternatively, a (finite) set also corresponds to a (finite) étale $\bar{k}$-scheme. Therefore it is enough to show the corresponding statement that replaces $k_{\text {prozar }}$ with $\bar{k}_{\text {proet }}$, Fin with FEt $/ \bar{k}$ and Ab with Et $/ \bar{k}$. Let $\bar{f}:$ Spec $\bar{k}_{\text {proet }}^{\text {indrat }} \rightarrow \operatorname{Spec} \bar{k}_{\text {proet }}$ be the morphism defined by the identity. Then $\bar{f}_{*} \bar{f}^{*}=$ id on $D\left(\bar{k}_{\text {proet }}\right)$, so $\bar{f}^{*}: D\left(\bar{k}_{\text {proet }}\right) \rightarrow$ $D\left(\bar{k}_{\text {proet }}^{\text {indrat }}\right)$ is fully faithful. The fully faithful embeddings

$$
D^{b}(\operatorname{IPAlg} / \bar{k}), D^{b}(\mathrm{Et} / \bar{k}) \hookrightarrow D^{b}\left(\bar{k}_{\text {proet }}^{\text {indrat }}\right)
$$

in (2.3.4) then restrict to fully faithful embeddings

$$
D^{b}(\mathrm{IPFEt} / \bar{k}), D^{b}(\mathrm{Et} / \bar{k}) \hookrightarrow D^{b}\left(\bar{k}_{\text {proet }}\right) .
$$

(b) It is enough to show the statements for $A \in \mathrm{Alg} / k$. By Lang's theorem Ser88, VI, Prop. 3], we have an exact sequence

$$
0 \rightarrow A(k) \rightarrow A \stackrel{F-1}{\rightarrow} A \rightarrow \pi_{0}(A)_{F} \rightarrow 0,
$$

where $\pi_{0}(A)_{F}$ is the coinvariants under $F$. The groups $A(k), \pi_{0}(A)_{F}$ are finite constant groups and, in particular, locally of finite presentation. Hence $\Gamma\left(k_{W}, A\right)=$ $A(k) \in$ Fin and $H^{1}\left(k_{W}, A\right)=\pi_{0}(A)_{F} \in$ Fin.

Since $F=1$ on $\mathbb{Z}$, we have $R \Gamma\left(k_{W}, \mathbb{Z}\right)=\mathbb{Z} \oplus \mathbb{Z}[-1]$. Hence for any $A \in D\left(k_{\text {proet }}^{\text {indrat }}\right)$, we have natural morphisms

$$
\begin{aligned}
R \Gamma\left(k_{W}, A^{\mathrm{SD}}\right) & =R \Gamma\left(k_{W}, R \mathbf{H o m}_{k_{\mathrm{proet}}^{\text {indrat }}}(A, \mathbb{Z})\right) \\
& \rightarrow R \mathbf{H o m}_{k_{\text {prozar }}}\left(R \Gamma\left(k_{W}, A\right), R \Gamma\left(k_{W}, \mathbb{Z}\right)\right) \\
& \left.\rightarrow R \mathbf{H o m}_{k_{\text {prozar }}}\left(R \Gamma\left(k_{W}, A\right), \mathbb{Z}\right)\right)[-1] \\
& =R \Gamma\left(k_{W}, A\right)^{\mathrm{LD}}[-1] \\
& \leftarrow R \Gamma\left(k_{W}, A\right)^{\mathrm{PD}}[-2],
\end{aligned}
$$

where we denote $B^{\mathrm{LD}}=R \mathbf{H o m}_{k_{\text {prozar }}}(B, \mathbb{Z})($ linear dual $)$ and $B^{\mathrm{PD}}=R \mathbf{H o m}_{k_{\text {prozar }}}(B, \mathbb{Q} / \mathbb{Z})$ (Pontryagin dual) for $B \in D\left(k_{\text {prozar }}\right)$.

Proposition (10.4). If $A \in \mathrm{IPAlg} / k$, then the above defined morphisms

$$
R \Gamma\left(k_{W}, A^{\mathrm{SD}}\right) \rightarrow R \Gamma\left(k_{W}, A\right)^{\mathrm{LD}}[-1] \leftarrow R \Gamma\left(k_{W}, A\right)^{\mathrm{PD}}[-2]
$$

are isomorphisms. If $A \in \mathrm{FGEt} / k$, then the morphism

$$
R \Gamma\left(k_{W}, A^{\mathrm{SD}}\right) \rightarrow R \Gamma\left(k_{W}, A\right)^{\mathrm{LD}}[-1]
$$

is an isomorphism. 
Proof. For the moment, let $A \in \mathrm{Ab}\left(k_{\text {proet }}^{\text {indrat }}\right)$ be arbitrary. We use the identification $\operatorname{Spec} \bar{k}_{\text {proet }} \cong \operatorname{Spec} k_{\text {prozar }}$. Hence we view the map $f$ used in (10.2) as a continuous map of sites Spec $k_{\text {proet }}^{\text {indrat }} \rightarrow$ Spec $\bar{k}_{\text {proet }}$ defined by the identity. Let $g:$ Spec $k_{\text {proet }}^{\text {indrat }} \rightarrow$ Spec $k_{\text {proet }}$ be the morphism defined by the identity. Let $j:$ Spec $\bar{k}_{\text {proet }} \rightarrow$ Spec $k_{\text {proet }}$ be the natural morphism (defined by the functor $\left.k^{\prime} \in k_{\text {proet }} \mapsto k^{\prime} \otimes \bar{k} \in \bar{k}_{\text {proet }}\right)$. The pullback $j^{*}$ is the restriction functor $\left.\right|_{\text {Spec } \bar{k}}$. Hence $f_{*}=j^{*} g_{*}$ as functors $D\left(k_{\text {proet }}^{\text {indrat }}\right) \rightarrow D\left(\bar{k}_{\text {proet }}\right)$.

We have

$$
R \Gamma\left(k_{W}, A^{\mathrm{SD}}\right)=j^{*} g_{*} R \mathbf{H o m}_{k_{\mathrm{proet}}^{\text {indrat }}}([A \stackrel{F-1}{\rightarrow} A], \mathbb{Z}) .
$$

On the other hand, the restriction $j^{*}$ commutes with derived sheaf-Hom's:

$$
j^{*} R \mathbf{H o m}_{k_{\text {proet }}}(B, C)=R \mathbf{H o m}_{\bar{k}_{\text {proet }}}\left(j^{*} B, j^{*} C\right)
$$

([KS06, Prop. 18.4.6]). Together with the adjunction $g^{*} \leftrightarrow g_{*}$ and $j^{*} \mathbb{Z}=\mathbb{Z}$ and $g_{*} \mathbb{Z}=\mathbb{Z}$, we have

$$
\begin{aligned}
R \Gamma\left(k_{W}, A\right)^{\mathrm{LD}}[-1] & =R \mathbf{H o m}_{\overline{\mathrm{proet}}_{\text {proe }}}\left(j^{*} g_{*}[A \stackrel{F-1}{\rightarrow} A], \mathbb{Z}\right) \\
& =j^{*} g_{*} R \mathbf{H o m}_{k_{\mathrm{proet}}^{\text {indrat }}}\left(g^{*} g_{*}[A \stackrel{F-1}{\rightarrow} A], \mathbb{Z}\right) .
\end{aligned}
$$

Hence the morphism $R \Gamma\left(k_{W}, A^{\mathrm{SD}}\right) \rightarrow R \Gamma\left(k_{W}, A\right)^{\mathrm{LD}}[-1]$ is an isomorphism if $g^{*} g_{*}[A \rightarrow A]=[A \rightarrow A]$. For a profinite set $X$ viewed as an object of $k^{\text {indrat }}$, we have $g^{*} g_{*} X=g^{*} X=X$ since $X$ is representable in $k_{\text {proet }}$. The same is true if $X$ is an ind-object of profinite sets (in particular, a set) since both $g^{*}$ and $g_{*}$ commute with filtered direct limits. We have $[A \rightarrow A] \in D^{b}$ (IPFin) if $A \in \operatorname{IPAlg} / k$ by (10.3) (b), and $[A \rightarrow A] \in D^{b}(\mathrm{Ab})$ if $A \in \mathrm{FGEt} / k$. Hence $g^{*} g_{*}[A \rightarrow A]=[A \rightarrow A]$ in either case. (Note that $g^{*} g_{*} \mathbf{G}_{a}$ is not $\mathbf{G}_{a}$ but the étale group whose group of geometric points is the discrete $\mathrm{Gal}(\bar{k} / k)$-modules of the additive group $\bar{k})$.

For the isomorphism $R \Gamma\left(k_{W}, A\right)^{\mathrm{PD}}[-2]=R \Gamma\left(k_{W}, A\right)^{\mathrm{LD}}[-1]$ for $A \in \operatorname{IPAlg} / k$, it is enough to show that $R \mathbf{H o m}_{\bar{k}_{\text {proet }}}(B, \mathbb{Q})=0$ for any $B \in \mathrm{IPFin}$. We can see this by the same proof as (2.3.1) (d) or by reducing to it by $R \operatorname{Hom}_{\bar{k}_{\text {proet }}}(B, \mathbb{Q})=$ $\bar{f}_{*} R \mathbf{H o m}_{\bar{k}_{\text {proet }}^{\text {indrat }}}(B, \mathbb{Q})=0$, where $\bar{f}:$ Spec $\bar{k}_{\text {proet }}^{\text {indrat }} \rightarrow \bar{k}_{\text {proet }}$ is the morphism defined by the identity.

Let $A \in \mathrm{Ab}\left(K_{\mathrm{fppf}} / k_{\mathrm{et}}^{\text {indrat }}\right)$. Assume that $R \boldsymbol{\Gamma}(K, A)$ is $\mathrm{P}$-acyclic. Then

$$
R \Gamma\left(k_{W}, R \boldsymbol{\Gamma}(K, A)\right)(k)=\left[R \Gamma\left(\hat{K}^{\mathrm{ur}}, A\right) \stackrel{F-1}{\rightarrow} R \Gamma\left(\hat{K}^{\mathrm{ur}}, A\right)\right][-1] .
$$

This is equal to $R \Gamma\left(W_{K}, A(L)\right)$ when the fppf cohomology $R \Gamma\left(\hat{K}^{\mathrm{ur}}, A\right)$ agrees with the étale cohomology $R \Gamma\left(\hat{K}_{\mathrm{et}}^{\mathrm{ur}}, A\right)$ (such as when $A$ is a smooth group scheme over $K)$, where $W_{K}$ is the Weil group of $K$ and $L$ a separable closure of $\hat{K}^{\mathrm{ur}}$. For this reason, we denott19

$$
\begin{gathered}
R \Gamma\left(K_{W}, A\right)=R \Gamma\left(k_{W}, R \tilde{\boldsymbol{\Gamma}}(K, A)\right) \in D\left(k_{\text {prozar }}\right), \\
H^{n}\left(K_{W}, A\right)=H^{n} R \Gamma\left(K_{W}, A\right) \in \operatorname{Ab}\left(k_{\text {prozar }}\right)
\end{gathered}
$$

\footnotetext{
19 The notation $R \Gamma\left(K_{W}, \cdot\right)$ reads "Weil-fppf cohomology of the scheme Spec $K$ ", while $R \Gamma\left(W_{K}, \cdot\right)$ is group cohomology of the topological group $W_{K}$. It is not good to choose the latter as general notation for the cohomology theory defined here since fppf cohomology is not always described as group cohomology.
} 
for any $A \in \mathrm{Ab}\left(K_{\mathrm{fppf}} / k_{\mathrm{et}}^{\text {indrat }}\right)$. Whenever $R \boldsymbol{\Gamma}(K, A)$ is $\mathrm{P}$-acyclic and in $D^{b}(\mathrm{IPAlg} / k)$ (resp. $\left.D^{b}(\mathrm{Et} / k)\right)$, which happens in many cases by (3.4.3), the complex $R \Gamma\left(K_{W}, A\right)$ is thus an enhancement of the Weil group cohomology of $K$ as a complex of indprofinite groups (resp. finitely generated abelian groups). The above proposition says that the Serre duality for $R \tilde{\boldsymbol{\Gamma}}(K, A)$ is translated into the linear duality or Pontryagin duality for $R \Gamma\left(K_{W}, A\right)$ up to shift.

For example, let $A$ be an abelian variety over $K$. Then our duality theorem (4.1.2) and (4.2.2) show that

$$
\left[\stackrel{\lim }{n}_{n} \boldsymbol{\Gamma}\left(K, A^{\vee}\right) \rightarrow R \boldsymbol{\Gamma}\left(K, A^{\vee}\right)\right]=R \boldsymbol{\Gamma}(K, A)^{\mathrm{SD}}[1] .
$$

Apply $R \Gamma\left(k_{W}, \cdot\right)$. If $\boldsymbol{\Gamma}\left(K, A^{\vee}\right)_{\mathrm{sAb}}$ denotes the semi-abelian quotient of $\boldsymbol{\Gamma}\left(K, A^{\vee}\right)_{0}$, which is quasi-algebraic, then $R \Gamma\left(k_{W}, \boldsymbol{\Gamma}\left(K, A^{\vee}\right)_{\mathrm{sAb}}\right)$ is finite by (10.3). Therefore

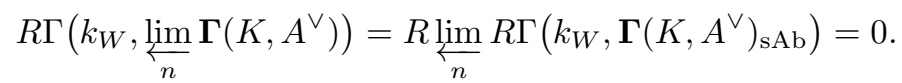

Hence (10.4) shows that we have an isomorphism

$$
R \Gamma\left(K_{W}, A^{\vee}\right)=R \Gamma\left(K_{W}, A\right)^{\mathrm{PD}}[-1]
$$

in $D^{b}$ (IPFin). The description of $\mathbf{H}^{n}(K, A)$ in (3.4.3) (d) shows that $H^{0}\left(K_{W}, A\right)=$ $A(K)$ is a profinite group, $H^{1}\left(K_{W}, A\right)=H^{1}(K, A)$ an indfinite (i.e., torsion) group, and $H^{n}\left(K_{W}, A\right)=0$ for $n \neq 0,1$. This recovers Tate-Milne's duality Tat58, Mil70] Mil72] (see also [Mil06, I, Cor. 3.4 and III, Thm. 7.8]).

If $T$ is a torus over $K$, then the same procedure gives isomorphisms

$$
\begin{aligned}
& R \Gamma\left(K_{W}, T^{\mathrm{CD}}\right)=R \Gamma\left(K_{W}, T\right)^{\mathrm{LD}}[-1] \\
& R \Gamma\left(K_{W}, T\right)=R \Gamma\left(K_{W}, T^{\mathrm{CD}}\right)^{\mathrm{LD}}[-1]
\end{aligned}
$$

in $D^{b}\left(k_{\text {prozar }}\right)$. We see that $H^{0}\left(K_{W}, T\right)=T(K)$ is an extension of a finite free abelian group by a profinite group, $H^{1}\left(K_{W}, T\right)$ a finitely generated abelian group and $H^{n}\left(K_{W}, T\right)=0$ for $n \neq 0,1$. We also see that $H^{0}\left(K_{W}, T^{\mathrm{CD}}\right)$ and $H^{1}\left(K_{W}, T^{\mathrm{CD}}\right)$ are finitely generated abelian groups, $H^{2}\left(K_{W}, T^{\mathrm{CD}}\right)=H^{1}\left(K, \hat{T}_{\text {tor }}\right)$ an indfinite (i.e. torsion) group and $H^{n}=0$ for $n \neq 0,1,2$. Let $C$ be an algebraically closed field of characteristic zero. We view $C^{\times}$as a constant group scheme over $k$ or $K$ and let $\hat{T}(C)=T^{\mathrm{CD}} \otimes_{\mathbb{Z}} C^{\times}$be the $C$-value points of the dual torus. Taking $\otimes_{\mathbb{Z}}^{L} C^{\times}$of the above isomorphism, we have

$$
R \Gamma\left(K_{W}, \hat{T}(C)\right)=R \mathbf{H o m}_{k_{\text {prozar }}}\left(R \Gamma\left(K_{W}, T\right), C^{\times}\right)[-1]
$$

by the same method as the proof of (8.3). From $H^{1}$, we get

$$
H^{1}\left(K_{W}, \hat{T}(C)\right)=\mathbf{H o m}_{k_{\text {prozar }}}\left(\Gamma(K, T), C^{\times}\right) .
$$

This recovers the local Langlands correspondence for tori Lan97.

If $M$ is a 1-motive over $K$, then we have

$$
R \Gamma\left(k_{W}, R \boldsymbol{\Gamma}(K, M)^{\mathrm{SDSD}}\right)=R \Gamma\left(k_{W}, R \boldsymbol{\Gamma}(K, M)\right)
$$

by reducing it to the previous cases of abelian varieties, tori and lattices by the weight filtration, and an isomorphism

$$
R \Gamma\left(K_{W}, M^{\vee}\right)=R \Gamma\left(K_{W}, M\right)^{\mathrm{LD}}
$$


in $D^{b}\left(k_{\text {prozar }}\right)$. This case, it is a little bit complicated to describe each $H^{n}\left(K_{W}, M\right)$ due to its possible uniquely divisible part. This is essentially a result of HarariSzamuely [HS05, Thm. 2.3].

If $N$ is a finite flat group scheme over $K$, then the Bégueri-Bester-Kato duality

$$
R \boldsymbol{\Gamma}\left(K, N^{\mathrm{CD}}\right)=R \boldsymbol{\Gamma}(K, N)^{\mathrm{SD}}
$$

(see Thm. (5.2.2.1) and Suz13, Thm. 2.7.1] for this form of the statement) implies

$$
R \Gamma\left(K_{W}, N^{\mathrm{CD}}\right)=R \Gamma\left(K_{W}, N\right)^{\mathrm{PD}}[-2]
$$

in $D^{b}$ (IPFin). We see that $H^{0}\left(K_{W}, N\right)=H^{0}(K, N)$ and $H^{2}\left(K_{W}, N\right)=H^{2}(K, N)$ are finite, $H^{1}\left(K_{W}, N\right)$ the group $H^{1}(K, N)$ endowed with an ind-profinite group structure, and $H^{n}=0$ for $n \neq 0,1,2$. If $K$ has mixed characteristic, then $H^{1}\left(K_{W}, N\right)$ is finite. This recovers Tate-Shatz's duality [Ser02, II, §5, Thm. 2], Sha64.

Remark (10.5). As in Sha64], the group $H^{1}(K, N)$ for equal characteristic $K$ is not only ind-profinite but locally compact (see also [Mil06, III, §6] and [Čes15]), i.e., it can be written as a filtered union $\bigcup B_{\lambda}$ of profinite groups such that $B_{\mu} / B_{\lambda}$ is finite for any $\mu \geq \lambda$. In our setting, we can deduce this from the fact (true for general perfect $k$ ) that $\mathbf{H}^{1}(K, N) \in \mathrm{IPAlg} / k$ can be written as a filtered union $\bigcup C_{\lambda}$ of proalgebraic groups such that $C_{\mu} / C_{\lambda}$ is quasi-algebraic for any $\mu \geq \lambda$. For the moment, let us say such an ind-proalgebraic group algebraically growing. To see that $\mathbf{H}^{1}(K, N)$ is algebraically growing, note that this is true for $N=\alpha_{p}, \mu_{p}, \mathbb{Z} / n \mathbb{Z}$ as seen from the proof of (3.4.3). Let $0 \rightarrow C_{1} \rightarrow C_{2} \rightarrow C_{3} \rightarrow 0$ be an exact sequence in IPAlg/k. If $C_{2}$ is algebraically growing, so is $C_{1}$. If $C_{1}$ and $C_{3}$ are both algebraically growing, so is $C_{2}$. If $C_{2}$ is algebraically growing and $C_{1} \in \mathrm{PAlg} / k$, then $C_{3}$ is algebraically growing. Then the general finite flat group case follows from the proof of the same proposition.

\section{Appendix A. The PRO-FPPF TOPOLOGY FOR PROALGEBRAIC PROSChEMES}

In this appendix, we briefly explain how (2.3.1), originally proved in [Suz13, §3] for affine proalgebraic groups $A$, can be extended for arbitrary proalgebraic groups. For abelian varieties, the same proof as [Suz13] works. Although the general case including infinite products of abelian varieties is not needed in the main body of this paper, we treat the general case in this appendix for completeness. The problem is that infinite products of abelian varieties are not schemes, but proschemes. Let $k^{\text {proalg }}$ be the procategory of the category of quasi-algebraic $k$-schemes (i.e., perfections of $k$-schemes of finite type). We call an object of $k^{\text {proalg }}$ a proalgebraic $k$-proscheme. Examples are infinite products of perfections of proper varieties.

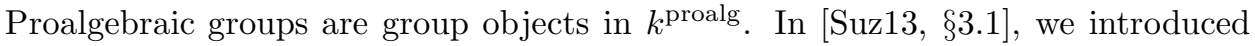
the pro-fppf topology on the category of perfect affine $k$-schemes. Below we extend it to the category $k^{\text {proalg }}$ of proalgebraic $k$-proschemes in the paragraph after (A.3). We also show in (A.4) that the pro-fppf site of proalgebraic $k$-proschemes has an equivalent topos to the pro-fppf site of perfect affine $k$-schemes. Then most part of [Suz13, §3] goes without large modifications.

Generalizing [Suz13, §3.1] from the affine case, we say that a morphism of quasicompact quasi-separated perfect $k$-schemes $Y \rightarrow X$ is of finite presentation if it is the perfection of a $k$-scheme morphism $Y_{0} \rightarrow X$ of finite presentation in the usual sense. If moreover $Y_{0} \rightarrow X$ can be chosen to be (faithfully) flat, we say that 
$Y \rightarrow X$ is (faithfully) flat of finite presentation (in the perfect sense) 20 A quasicompact quasi-separated perfect $k$-scheme can naturally be viewed as an object of $k^{\text {proalg }}$ since it is a filtered inverse limit of quasi-algebraic $k$-schemes with affine transition morphisms (use the absolute noetherian approximation TT90, Thm. C.9] and apply the perfection). We say that a morphism $Y \rightarrow X$ in $k^{\text {proalg }}$ is of finite presentation if it can be written as the base change of a morphism of finite presentation $Y_{0} \rightarrow X_{0}$ in the above perfect sense between quasi-compact quasiseparated perfect $k$-schemes by some morphism $X \rightarrow X_{0}$ in $k^{\text {proalg }}$. If $Y_{0} \rightarrow X_{0}$ can be chosen to be (faithfully) flat of finite presentation (in the above perfect sense), then we say that $Y \rightarrow X$ is (faithfully) flat of finite presentation. We say that a morphism $Y \rightarrow X$ in $k^{\text {proalg }}$ is (faithfully) flat of profinite presentation if there exists a filtered inverse system $\left\{Y_{\lambda}\right\}$ in $k^{\text {proalg }}$ such that each morphism $Y_{\lambda} \rightarrow X$ is (faithfully) flat of finite presentation and $Y$ is isomorphic to $\lim _{\lambda} Y_{\lambda}$ over $X$ in

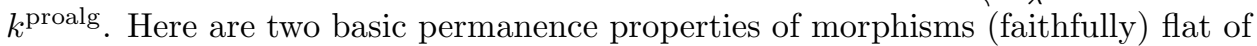
finite presentation.

Proposition (A.1). If a morphism $Y \rightarrow X$ in $k^{\text {proalg }}$ is (faithfully) flat of finite presentation, then it can be written as the base change of a morphism $Y_{0} \rightarrow X_{0}$ (faithfully) flat of finite presentation between quasi-algebraic $k$-schemes by some morphism $X \rightarrow X_{0}$ in $k^{\text {proalg }}$.

Proof. We may assume that $X$ and $Y$ are quasi-compact quasi-separated perfect $k$-schemes. Write $Y \rightarrow X$ as the perfection of a morphism $Y_{0}^{\prime} \rightarrow X$ (faithfully) flat of finite presentation in the usual sense. By [TT90, Thm. C.9], we can write $X$ as a filtered inverse limit of algebraic $k$-schemes with affine transition morphisms. Hence the permanence property of flatness (resp. surjectivity) under passage to limits Gro66, Thm. 11.2.6] (resp. Gro66, Thm. 8.10.5]) shows that $Y_{0}^{\prime} \rightarrow X$ can be written as the base change of a morphism $Y_{00} \rightarrow X_{00}$ (faithfully) flat of finite presentation in the usual sense between algebraic $k$-schemes by some morphism $X \rightarrow X_{00}$. Let $Y_{0} \rightarrow X_{0}$ be the perfection of $Y_{00} \rightarrow X_{00}$, which is (faithfully) flat of finite presentation (in the perfect sense) between quasi-algebraic $k$-schemes. Its base change by $X \rightarrow X_{0}$ is $Y \rightarrow X$.

Proposition (A.2). Let $\left\{X_{\lambda}\right\}$ be a filtered inverse system in $k^{\text {proalg }}$ with inverse limit $X$. Let $Y \rightarrow X$ be a (faithfully) flat morphism of finite presentation in $k^{\text {proalg }}$. Then there exist an index $\lambda$, an object $Y_{\lambda}$ of $k^{\text {proalg }}$ and a (faithfully) flat morphism of finite presentation $Y_{\lambda} \rightarrow X_{\lambda}$ in $k^{\text {proalg }}$ such that the base change of $Y_{\lambda} \rightarrow X_{\lambda}$ by $X \rightarrow X_{\lambda}$ gives the original morphism $Y \rightarrow X$.

Proof. As in the previous proposition, let $Y_{0} \rightarrow X_{0}$ be a (faithfully) flat morphism of finite presentation between quasi-algebraic $k$-schemes whose base change to $X$ by some morphism $X \rightarrow X_{0}$ gives the morphism $Y \rightarrow X$. Then the morphism $X \rightarrow X_{0}$ factors through some $X_{\lambda}$. Let $Y_{\lambda} \rightarrow X_{\lambda}$ be the base change of $Y_{0} \rightarrow X_{0}$ by the morphism $X_{\lambda} \rightarrow X_{0}$. Then $Y_{\lambda} \rightarrow X_{\lambda}$ is (faithfully) flat of finite presentation, and its base change to $X$ is the morphism $Y \rightarrow X$.

20 The notion of morphisms of finite presentation in the sense here is Zariski local in the source and the target, as shown in Ser60, $\S 1$, Cor. to Prop. 9] when $X=\operatorname{Spec} k$ and $Y$ separated, and in BS17 Prop. 3.13] in general. But it is not clear whether the notion of flat morphisms of finite presentation is Zariski local or not. The definition given here is sufficient for defining a nice enough category of sheaves. 
A fact already used above is that a base change of a morphism (faithfully) flat of finite presentation is also. The same is true for a morphism (faithfully) flat of profinite presentation. For composites, we have the following.

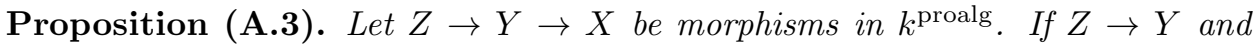
$Y \rightarrow X$ are (faithfully) flat of finite presentation, then so is $Z \rightarrow X$. If $Z \rightarrow Y$ and $Y \rightarrow X$ are (faithfully) flat of profinite presentation, then so is $Z \rightarrow X$.

Proof. Assume that $Z \rightarrow Y$ and $Y \rightarrow X$ are (faithfully) flat of finite presentation. We first treat that case that $X, Y, Z$ are quasi-compact quasi-separated perfect $k$ schemes. Write $Z \rightarrow Y$ (resp. $Y \rightarrow X$ ) as the perfection of a morphism $Z_{0} \rightarrow Y$ (resp. $Y_{0} \rightarrow X$ ) (faithfully) flat of finite presentation in the usual sense. For any $n \geq 0$, let $Y_{0}^{(n)}$ be the scheme $Y_{0}$ with a new $k$-scheme structure given by the composite

$$
Y_{0} \rightarrow \operatorname{Spec} k \stackrel{\sim}{\rightarrow} \operatorname{Spec} k,
$$

where the first morphism is the original $k$-scheme structure morphism of $Y_{0}$ and the second morphism is the $p^{n}$-th power absolute Frobenius of Spec $k$. The scheme $Y_{0}^{(n)}$ is (faithfully) flat of finite presentation over $X\left(=X^{(n)}\right)$ in the usual sense. Since $Y=\lim _{n} Y_{0}^{(n)}$, the permanence property of flatness (resp. surjectivity) under passage to limits Gro66, Thm. 11.2.6] (resp. Gro66, Thm. 8.10.5]) shows that we can write $Z_{0} \rightarrow Y$ as the base change of some (faithfully) flat morphism of finite presentation $Z_{00} \rightarrow Y_{0}^{(n)}$ in the usual sense by the morphism $Y \rightarrow Y_{0}^{(n)}$. Then $Z \rightarrow X$ is the perfection of the composite $Z_{00} \rightarrow Y_{0}^{(n)} \rightarrow X$, which is (faithfully) flat of finite presentation in the usual sense. Hence $Z \rightarrow X$ is (faithfully) flat of finite presentation (in the perfect sense).

We treat the general case. Write $X$ as a filtered inverse limit of quasi-algebraic $k$ schemes $X_{\lambda}$. Write $Y \rightarrow X$ as the base change of a morphism $Y_{\lambda} \rightarrow X_{\lambda}$ (faithfully) flat of finite presentation (in the perfect sense) as in the previous proposition. Let $Y_{\mu}=Y_{\lambda} \times_{X_{\lambda}} X_{\mu}$ for $\mu \geq \lambda$. Then $Y=\lim _{\mu \geq \lambda} Y_{\mu}$. Write $Z \rightarrow Y$ as the base change of a morphism $Z_{\mu} \rightarrow Y_{\mu}$ (faithfully) flat of finite presentation as in the previous proposition. Then $Z \rightarrow X$ is the base change of the composite $Z_{\mu} \rightarrow Y_{\mu} \rightarrow X_{\mu}$, which is (faithfully) flat of finite presentation by the quasi-compact quasi-separated case treated earlier.

Next assume that $Z \rightarrow Y$ and $Y \rightarrow X$ are (faithfully) flat of profinite presentation. Write $Z$ (resp. $Y$ ) as a filtered inverse limit of morphisms $Z_{\mu} \rightarrow Y$ (resp. $Y_{\lambda} \rightarrow X$ ) (faithfully) flat of finite presentation. By a similar argument as [Swa98, Lem. 1.5], it is enough to show that any morphism $Z \rightarrow Z^{\prime}$ over $X$ to an object $Z^{\prime}$ over $X$ of finite presentation factors through an object over $X$ (faithfully) flat of finite presentation. The $X$-morphism $Z \rightarrow Z^{\prime}$ factors through some $Z_{\mu}$. Write the morphism $Z_{\mu} \rightarrow Y$ as the base change of some morphism $Z_{\lambda \mu} \rightarrow Y_{\lambda}$ (faithfully) flat of finite presentation as in the previous proposition. Let $Z_{\lambda^{\prime} \mu}=Z_{\lambda \mu} \times_{Y_{\lambda}} Y_{\lambda^{\prime}}$ for $\lambda^{\prime} \geq \lambda$. Then $Z_{\mu}=\lim _{\lambda^{\prime} \geq \lambda} Z_{\lambda^{\prime} \mu}$. Then the $X$-morphism $Z \rightarrow Z^{\prime}$ factors through $Z_{\lambda^{\prime} \mu}$ for some $\lambda^{\prime} \geq \lambda$. The composite $Z_{\lambda^{\prime} \mu} \rightarrow Y_{\lambda^{\prime}} \rightarrow X$ is (faithfully) flat of finite presentation by what we have proven above. Thus $Z \rightarrow X$ is (faithfully) flat of profinite presentation.

With this, we can define the perfect fppf site of proalgebraic $k$-proschemes, Spec $k_{\mathrm{fppf}}^{\text {proalg }}$, to be the category $k^{\text {proalg }}$ endowed with the topology where a covering $\left\{Y_{i} \rightarrow X\right\}$ is a finite family of morphisms such that $\bigsqcup Y_{i} \rightarrow X$ is faithfully 
flat of finite presentation (which implies that each $Y_{i} \rightarrow X$ is flat of finite presentation). Replacing "faithfully flat of finite presentation" by "faithfully flat of profinite presentation", we can define the perfect pro-fppf site of proalgebraic $k$-proschemes Spec $k_{\text {profppf }}^{\text {proalg }}$ in the same way.

As in the main text, let $k^{\text {perf }}$ be the category of perfect $k$-algebras or perfect affine $k$-schemes. The identity functor defines a morphism of sites Spec $k_{\text {profppf }}^{\text {proalg }} \rightarrow$ Spec $k_{\text {profppf }}^{\text {perf }}$.

Proposition (A.4). The morphism of sites Spec $k_{\text {profppf }}^{\text {proalg }} \rightarrow \operatorname{Spec} k_{\text {profppf }}^{\text {perf }}$ defined by the identity induces an equivalence on the topoi.

In particular, Ext groups and Ext sheaves between proalgebraic groups can be calculated using either Spec $k_{\text {proappf }}^{\text {proalg }}$ or Spec $k_{\text {profppf }}^{\text {perf }}$.

To prove the proposition, the key is that a proalgebraic $k$-proscheme is proZariski locally affine (A.5) below). This is trivial for a proalgebraic $k$-proscheme $X=\lim _{i} X_{i}$ indexed by integers $i \geq 1$ : we can find an affine "pro-Zariski" covering $Y=\varlimsup_{i} Y_{i}$ of $X$ by successively taking affine Zariski coverings $Y_{1} \rightarrow X_{1}, Y_{2} \rightarrow$ $Y_{1} \times_{X_{1}} X_{2}, Y_{3} \rightarrow Y_{2} \times_{X_{2}} X_{3}$ and so on. Here we say that a surjective morphism of schemes $Y \rightarrow Z$ is an (affine) Zariski covering if there exists a decomposition $Y=$ $\sqcup Y_{\lambda}$ into disjoint (affine) open subschemes such that the composite $Y_{\lambda} \rightarrow Y \rightarrow Z$ is an open immersion for any $\lambda$. The difficulties thus come from the complexity of the index set.

Recall that a poset (partially ordered set) is a pre-ordered set such that $\lambda \leq \lambda^{\prime} \leq$ $\lambda$ implies $\lambda=\lambda^{\prime}$. A pre-ordered set $\Lambda$ is said to be cofinite if for every $\lambda \in \Lambda$, there are only finitely many elements $\leq \lambda$. An element $\lambda$ of a pre-ordered set is said to be greatest if $\lambda^{\prime} \leq \lambda$ for any $\lambda^{\prime}$. If $\Lambda$ is a directed set without a greatest element and $M$ is the set of all finite subsets $\Lambda^{\prime} \subset \Lambda$ having a unique greatest element $\max \left(\Lambda^{\prime}\right)$, then $M$ is a cofinite directed poset by inclusion, and the map $\Lambda^{\prime} \mapsto \max \left(\Lambda^{\prime}\right)$ from $M$ to $\Lambda$ is cofinal (which is implicit in the proof of AGV72, I, Prop. 8.1.6]). If $\Lambda$ has a greatest element $\lambda$, then $\{\lambda\} \hookrightarrow \Lambda$ is cofinal. Therefore every filtered inverse system in a category is isomorphic (in the procategory) to a system indexed by a cofinite directed poset (again implicit in the proof of [AGV72, loc. cit.]). For a cofinite poset $\Lambda$ and an element $\lambda \in \Lambda$, we define $n(\lambda)$ to be the largest integer $n \geq 1$ such that there exists a chain $\lambda_{1}<\cdots<\lambda_{n-1}<\lambda$ of elements of $\Lambda$ (so $n(\lambda)=1$ if $\lambda$ is minimal). This function satisfies the property that if $\lambda \leq \lambda^{\prime}$, then $\lambda=\lambda^{\prime}$ or $n(\lambda)<n\left(\lambda^{\prime}\right)$.

Proposition (A.5). Let $\left\{X_{\lambda}\right\}_{\lambda \in \Lambda}$ be an inverse system of quasi-compact quasiseparated $k$-schemes. Assume that the index set $\Lambda$ is a cofinite poset ${ }^{21}$ Then there exists an inverse system $\left\{Y_{\lambda}\right\}$ of affine $k$-schemes and a system $\left\{Y_{\lambda} \rightarrow X_{\lambda}\right\}$ of $k$-scheme morphisms such that each $Y_{\lambda} \rightarrow X_{\lambda}$ is a Zariski covering.

Proof. We will construct a system

$$
\left\{\cdots \rightarrow Y_{\lambda}^{n+1} \rightarrow Y_{\lambda}^{n} \rightarrow \cdots \rightarrow Y_{\lambda}^{0}=X_{\lambda}\right\}_{\lambda}
$$

of sequences of Zariski coverings of quasi-compact quasi-separated $k$-schemes such that for each $n$ and $\lambda$ with $n(\lambda) \leq n$, the scheme $Y_{\lambda}^{n}$ is affine and the morphisms $\cdots \rightarrow Y_{\lambda}^{n+1} \rightarrow Y_{\lambda}^{n}$ after the $n$-th term are isomorphisms. Then, letting $Y_{\lambda}=Y_{\lambda}^{n}$ for $n(\lambda) \leq n$, we will obtain a required system $\left\{Y_{\lambda}\right\}$ of coverings.

\footnotetext{
${ }^{21}$ We do not need to assume that $\Lambda$ is directed, though we only need the directed case below.
} 
Fix an integer $n \geq 0$. Suppose that we have such a sequence $\left\{Y_{\lambda}^{n} \rightarrow \cdots \rightarrow Y_{\lambda}^{0}\right\}_{\lambda}$ up to $n$. For each $\lambda$ with $n(\lambda)=n+1$, we choose an affine Zariski covering $Y_{\lambda}^{n+1} \rightarrow Y_{\lambda}^{n}$. For general $\lambda$, we define

$$
Y_{\lambda}^{n+1}=\prod_{\substack{\lambda_{\lambda}^{n} \leq \lambda, n\left(\lambda^{\prime}\right)=n+1 \\ \lambda^{\prime}=n}}\left(Y_{\lambda^{\prime}}^{n+1} \times_{Y_{\lambda^{\prime}}^{n}} Y_{\lambda}^{n}\right),
$$

where $\prod_{Y_{\lambda}^{n}}$ denotes the fiber product over $Y_{\lambda}^{n}$. This is a finite product since $\Lambda$ is cofinite. The property of the function $n(\lambda)$ above shows that this definition is consistent with $Y_{\lambda}^{n+1}$ taken early for $n(\lambda)=n+1$, and the natural morphism $Y_{\lambda}^{n+1} \rightarrow Y_{\lambda}^{n}$ is an isomorphism for $n(\lambda) \leq n$. The morphism $Y_{\lambda}^{n+1} \rightarrow Y_{\lambda}^{n}$ for general $\lambda$ is a Zariski covering since it is a finite fiber product of Zariski coverings. Each $Y_{\lambda}^{n+1}$ is a quasi-compact quasi-separated $k$-scheme since it is a fiber product of such. The morphisms $Y_{\lambda}^{n+1} \rightarrow Y_{\lambda}^{n}$ form a system. By induction, we obtain a required sequence.

We also need to compare the topology on $k^{\text {perf }}$ induced from Spec $k_{\text {profppf }}^{\text {proalg }}$ and the topology of Spec $k_{\text {profppf }}^{\text {perf }}$ defined in 2.1 The following shows that they agree up to refinement.

Proposition (A.6). If a morphism Spec $S \rightarrow$ Spec $R$ between perfect affine (hence quasi-compact quasi-separated) $k$-schemes is (faithfully) flat of profinite presentation in the sense of this appendix, then there exists a homomorphism $S \rightarrow T$ faithfully flat of ind-finite presentation in the sense of 92.1 between perfect $k$-algebra 22 such that $R \rightarrow T$ is (faithfully) flat of ind-finite presentation in the sense of $\sqrt{2.1}$.

Proof. Let $X=\operatorname{Spec} R$ and $Y=\operatorname{Spec} S$. Write $Y \rightarrow X$ as a filtered inverse limit of morphisms $Y_{\lambda} \rightarrow X$ (faithfully) flat of finite presentation in the sense of this appendix with $Y_{\lambda}$ quasi-compact quasi-separated perfect $k$-schemes. We may assume that the index set $\Lambda$ is a cofinite directed poset. Take a system of affine Zariski coverings $\left\{Z_{\lambda}=\operatorname{Spec} T_{\lambda} \rightarrow Y_{\lambda}\right\}$ as in the previous proposition. The scheme $Z_{\lambda}$ for any $\lambda$ is perfect since $Y_{\lambda}$ is so. For each $\lambda$, the composite $Z_{\lambda} \rightarrow Y_{\lambda} \rightarrow X$ is (faithfully) flat of finite presentation in the sense of this appendix by A.3. Therefore $Z_{\lambda} \rightarrow X$ can be written as the perfection of a morphism $Z_{\lambda_{0}} \rightarrow X$ (faithfully) flat of finite presentation in the usual sense, where $Z_{\lambda 0}$ is a priori a quasicompact quasi-separated $k$-scheme. Since $Z_{\lambda}$ is affine, we know that $Z_{\lambda 0}$ is actually affine by [TT90, Prop. C.6]. Hence $R \rightarrow T_{\lambda}$ is (faithfully) flat of finite presentation in the sense of 92.1 Thus $T=\lim T_{\lambda}$ is (faithfully) flat of ind-finite presentation over $R$ in the sense of $\$ 2.1$. Let $\overrightarrow{Z=} \operatorname{Spec} T=\lim Z_{\lambda}$. Then $Z=\lim Z_{\lambda} \times_{Y_{\lambda}} Y$ and $S \rightarrow T_{\lambda} \otimes_{S_{\lambda}} S$ is faithfully flat of finite presentation in the sense of 92.1 . Therefore $S \rightarrow T$ is faithfully flat of ind-finite presentation in the sense of 92.1 . (Of course this proof shows that $Z \rightarrow Y$ is actually a pro-Zariski covering.)

Proof of (A.4). Let $S=$ Spec $k_{\text {profppf }}^{\text {perf }}$ and $S^{\prime}=$ Spec $k_{\text {profppf }}^{\text {proalg }}$. Let $S^{\prime \prime}$ be the site on $k^{\text {perf }}$ with topology induced from Spec $k_{\text {profppf }}^{\text {proalg }}$ AGV72, Exp. II, §3]. We have morphisms $S^{\prime} \rightarrow S^{\prime \prime} \rightarrow S$ of sites defined by the identity. A sieve on an object of $k^{\text {perf }}$ is a covering for $S$ if and only if it is for $S^{\prime \prime}$ by the previous proposition. Hence

\footnotetext{
22 This means that $S \rightarrow T$ is flat of ind-finite presentation in the sense of 2.1 and faithfully
} flat. 
$S^{\prime \prime} \stackrel{\sim}{\rightarrow} S$ as sites. Any object of $S^{\prime}$ is covered by an object of $S^{\prime \prime}$ by A.5. Hence $S^{\prime} \rightarrow S^{\prime \prime}$ induces an equivalence on the topoi by [AGV72, Exp. II, Thm. 4.1].

We can define the étale site Spec $k_{\text {et }}^{\text {proalg }}$ and the pro-étale site Spec $k_{\text {proet }}^{\text {proalg }}$ on the category $k^{\text {proalg }}$ in the same way. See Gro67, Prop. 17.7.8] for the necessary permanence property of étaleness under passage to limits. We can show that the morphism of sites Spec $k_{\text {proet }}^{\text {proalg }} \rightarrow$ Spec $k_{\text {proet }}^{\text {perf }}$ defined by the identity induces an equivalence on the topoi by the same proof as above.

We generalize some more definitions in [Suz13, §3]. A proalgebraic $k$-proscheme can be viewed as a locally ringed space since the category of locally ringed spaces has all inverse limits ([Tem11, Rmk. 2.1.1], Gil11, Cor. 5]). If $\left\{X_{\lambda}\right\}$ is a filtered inverse system of quasi-compact quasi-separated perfect $k$-schemes, then the underlying topological space of $X=\lim X_{\lambda}$ is the inverse limit of the underlying topological spaces of $X_{\lambda}$. The structure sheaf of $X$ is the direct limit of the pullbacks by $X \rightarrow X_{\lambda}$ of the structure sheaves of $X_{\lambda}$. A morphism $Y \rightarrow X$ in $k^{\text {proalg }}$ is faithfully flat of (pro)finite presentation if and only if it is flat of (pro)finite presentation and surjective on the underlying topological spaces. We say that $Y \rightarrow X$ is dominant if its image on the underlying topological spaces is dense in $X$. (This definition will be needed to generalize [Suz13, §3.6].) Taking $X_{\lambda}$ to be quasi-algebraic, we can define profinite sets of points of $X=\lim X_{\lambda}$ and, if the transition morphisms $X_{\mu} \rightarrow X_{\lambda}$ are flat (or more generally, send irreducible components of $X_{\mu}$ onto dense subsets of irreducible components of $X_{\lambda}$ ), the generic point of $X$, in the same way as [Suz13, Def. 3.2.1]. They are Spec's of ind-rational $k$-algebras.

Then all of the discussions and results in [Suz13, §3] work without modifications with perfect affine $k$-schemes generalized to proalgebraic $k$-proschemes and with affine proalgebraic groups generalized to arbitrary proalgebraic groups. This proves (2.3.1) in full generality.

\section{REFERENCES}

[AGV72] Théorie des topos et cohomologie étale des schémas. Tome 1: Théorie des topos. Lecture Notes in Mathematics, Vol. 269. Springer-Verlag, Berlin, 1972. Séminaire de Géométrie Algébrique du Bois-Marie 1963-1964 (SGA 4), Dirigé par M. Artin, A. Grothendieck, et J. L. Verdier. Avec la collaboration de N. Bourbaki, P. Deligne et B. Saint-Donat.

[AM76] M. Artin and J. S. Milne. Duality in the flat cohomology of curves. Invent. Math., 35:111129, 1976.

[Art62] M. Artin. Grothendieck topologies. Cambridge, Mass.: Harvard University. 133 p. (1962)., 1962.

[Art69] M. Artin. Algebraic approximation of structures over complete local rings. Inst. Hautes Études Sci. Publ. Math., (36):23-58, 1969.

[BB00] Alessandra Bertapelle and Siegfried Bosch. Weil restriction and Grothendieck's duality conjecture. J. Algebraic Geom., 9(1):155-164, 2000.

[Bég81] Lucile Bégueri. Dualité sur un corps local à corps résiduel algébriquement clos. Mém. Soc. Math. France (N.S.), (4):121, 1980/81.

[Ber01] Alessandra Bertapelle. On perfectness of Grothendieck's pairing for the $l$-parts of component groups. J. Reine Angew. Math., 538:223-236, 2001.

[Ber03] Alessandra Bertapelle. Local flat duality of abelian varieties. Manuscripta Math., 111(2):141-161, 2003.

[Bes78] Michal Bester. Local flat duality of abelian varieties. Math. Ann., 235(2):149-174, 1978.

[BGA13] Alessandra Bertapelle and Cristian D. González-Avilés. The Greenberg functor revisited. Preprint, arXiv:1311.0051v4, 2013.

[BGA15] Alessandra Bertapelle and Cristian D. González-Avilés. On the cohomology of tori over local fields with perfect residue field. Israel J. Math., 206(1):431-455, 2015. 
[BGA17] Alessandra Bertapelle and Cristian D. González-Avilés. On the perfection of schemes. Expositiones Mathematicae, 2017. DOI: 10.1016/j.exmath.2017.08.001.

[BIK13] Dave Benson, Srikanth B. Iyengar, and Henning Krause. Module categories for group algebras over commutative rings. J. K-Theory, 11(2):297-329, 2013. With an appendix by Greg Stevenson.

[BL02] Siegfried Bosch and Dino Lorenzini. Grothendieck's pairing on component groups of Jacobians. Invent. Math., 148(2):353-396, 2002.

[BLR90] Siegfried Bosch, Werner Lütkebohmert, and Michel Raynaud. Néron models, volume 21 of Ergebnisse der Mathematik und ihrer Grenzgebiete (3) [Results in Mathematics and Related Areas (3)]. Springer-Verlag, Berlin, 1990.

[Bos97] Siegfried Bosch. Component groups of abelian varieties and Grothendieck's duality conjecture. Ann. Inst. Fourier (Grenoble), 47(5):1257-1287, 1997.

[Bou98] Nicolas Bourbaki. Commutative algebra. Chapters 1-7. Elements of Mathematics (Berlin). Springer-Verlag, Berlin, 1998. Translated from the French, Reprint of the 1989 English translation.

[Bou03] Nicolas Bourbaki. Algebra II. Chapters 4-7. Elements of Mathematics (Berlin). SpringerVerlag, Berlin, 2003. Translated from the 1981 French edition by P. M. Cohn and J. Howie, Reprint of the 1990 English edition [Springer, Berlin; MR1080964 (91h:00003)].

[Bre70] Lawrence Breen. Extensions of abelian sheaves and Eilenberg-MacLane algebras. Invent. Math., 9:15-44, 1969/1970.

[Bre78] Lawrence Breen. Extensions du groupe additif. Inst. Hautes Études Sci. Publ. Math., (48):39-125, 1978

[Bro82] Kenneth S. Brown. Cohomology of groups, volume 87 of Graduate Texts in Mathematics. Springer-Verlag, New York-Berlin, 1982.

[BS15] Bhargav Bhatt and Peter Scholze. The pro-étale topology for schemes. Astérisque, (369):99-201, 2015.

[BS17] Bhargav Bhatt and Peter Scholze. Projectivity of the Witt vector affine Grassmannian. Inventiones mathematicae, 209(2):329-423, Aug 2017.

[Čes15] Kestutis Česnavičius. Topology on cohomology of local fields. Forum Math. Sigma, 3:e16, 55,2015

[Con12] Brian Conrad. Weil and Grothendieck approaches to adelic points. Enseign. Math. (2), 58(1-2):61-97, 2012.

[CR15] Clifton Cunningham and David Roe. From the function-sheaf dictionary to quasicharacters of p-adic tori. Journal of the Institute of Mathematics of Jussieu, pages 1-37, 2015.

[DG70] Michel Demazure and Pierre Gabriel. Groupes algébriques. Tome I: Géométrie algébrique, généralités, groupes commutatifs. Masson \& Cie, Éditeur, Paris; North-Holland Publishing Co., Amsterdam, 1970. Avec un appendice Corps de classes local par Michiel Hazewinkel.

[Gil11] William D. Gillam. Localization of ringed spaces. Advances in Pure Mathematics, 1(5):250-263, 2011.

[Gre61] Marvin J. Greenberg. Schemata over local rings. Ann. of Math. (2), 73:624-648, 1961.

[Gre66] Marvin J. Greenberg. Rational points in Henselian discrete valuation rings. Inst. Hautes Études Sci. Publ. Math., (31):59-64, 1966.

[Gro64] A. Grothendieck. Éléments de géométrie algébrique (rédigé avec la collaboration de Jean Dieudonné). IV. Étude locale des schémas et des morphismes de schémas. I. Inst. Hautes Études Sci. Publ. Math., (20):259, 1964.

[Gro66] A. Grothendieck. Éléments de géométrie algébrique (rédigé avec la collaboration de Jean Dieudonné). IV. Étude locale des schémas et des morphismes de schémas. III. Inst. Hautes Études Sci. Publ. Math., (28):255, 1966.

[Gro67] A. Grothendieck. Éléments de géométrie algébrique (rédigé avec la collaboration de Jean Dieudonné). IV. Étude locale des schémas et des morphismes de schémas IV. Inst. Hautes Études Sci. Publ. Math., (32):361, 1967.

[Gro72] Groupes de monodromie en géométrie algébrique. I. Lecture Notes in Mathematics, Vol. 288. Springer-Verlag, Berlin-New York, 1972. Séminaire de Géométrie Algébrique du BoisMarie 1967-1969 (SGA 7 I), Dirigé par A. Grothendieck. Avec la collaboration de M. Raynaud et D. S. Rim.

[HN11] Lars Halvard Halle and Johannes Nicaise. Motivic zeta functions of abelian varieties, and the monodromy conjecture. Adv. Math., 227(1):610-653, 2011. 
[HS05] David Harari and Tamás Szamuely. Arithmetic duality theorems for 1-motives. J. Reine Angew. Math., 578:93-128, 2005.

[Kat86] Kazuya Kato. Duality theories for the p-primary étale cohomology. I. In Algebraic and topological theories (Kinosaki, 1984), pages 127-148. Kinokuniya, Tokyo, 1986.

[Kat91] Kazuya Kato. Generalized class field theory. In Proceedings of the International Congress of Mathematicians, Vol. I, II (Kyoto, 1990), pages 419-428. Math. Soc. Japan, Tokyo, 1991.

[Koy93] Yoshihiro Koya. A generalization of Tate-Nakayama theorem by using hypercohomology. Proc. Japan Acad. Ser. A Math. Sci., 69(3):53-57, 1993.

[KS06] Masaki Kashiwara and Pierre Schapira. Categories and sheaves, volume 332 of Grundlehren der Mathematischen Wissenschaften [Fundamental Principles of Mathematical Sciences]. Springer-Verlag, Berlin, 2006.

[Lan97] R. P. Langlands. Representations of abelian algebraic groups. Pacific J. Math., (Special Issue):231-250, 1997. Olga Taussky-Todd: in memoriam.

[Lan02] Serge Lang. Algebra, volume 211 of Graduate Texts in Mathematics. Springer-Verlag, New York, third edition, 2002.

[McC86] William G. McCallum. Duality theorems for Néron models. Duke Math. J., 53(4):1093$1124,1986$.

[Mil70] J. S. Milne. Weil-Châtelet groups over local fields. Ann. Sci. École Norm. Sup. (4), 3:273$284,1970$.

[Mil72] J. S. Milne. Addendum: "Weil-Châtelet groups over local fields" (Ann. Sci. École Norm. Sup. (4) 3 (1970), 273-284). Ann. Sci. École Norm. Sup. (4), 5:261-264, 1972.

[Mil80] J. S. Milne. Étale cohomology, volume 33 of Princeton Mathematical Series. Princeton University Press, Princeton, N.J., 1980.

[Mil06] J. S. Milne. Arithmetic duality theorems. BookSurge, LLC, Charleston, SC, second edition, 2006.

[ML57] Saunders Mac Lane. Homologie des anneaux et des modules. In Colloque de topologie algébrique, Louvain, 1956, pages 55-80. Georges Thone, Liège, 1957.

[ML63] Saunders Mac Lane. Homology. Die Grundlehren der mathematischen Wissenschaften, Bd. 114. Academic Press, Inc., Publishers, New York; Springer-Verlag, Berlin-GöttingenHeidelberg, 1963.

[Nee01] Amnon Neeman. Triangulated categories, volume 148 of Annals of Mathematics Studies. Princeton University Press, Princeton, NJ, 2001.

[Pép14] Cédric Pépin. Dualité sur un corps local de caractéristique positive à corps résiduel algébriquement clos. Preprint, arXiv:1411.0742 $1,2014$.

[Roo06] Jan-Erik Roos. Derived functors of inverse limits revisited. J. London Math. Soc. (2), 73(1):65-83, 2006

[Šaf61] I. R. Šafarevič. Principal homogeneous spaces defined over a function field. Trudy Mat. Inst. Steklov., 64:316-346, 1961.

[Ser60] Jean-Pierre Serre. Groupes proalgébriques. Inst. Hautes Études Sci. Publ. Math., (7):67, 1960.

[Ser61] Jean-Pierre Serre. Sur les corps locaux à corps résiduel algébriquement clos. Bull. Soc. Math. France, 89:105-154, 1961.

[Ser79] Jean-Pierre Serre. Local fields, volume 67 of Graduate Texts in Mathematics. SpringerVerlag, New York, 1979. Translated from the French by Marvin Jay Greenberg.

[Ser88] Jean-Pierre Serre. Algebraic groups and class fields, volume 117 of Graduate Texts in Mathematics. Springer-Verlag, New York, 1988. Translated from the French.

[Ser02] Jean-Pierre Serre. Galois cohomology. Springer Monographs in Mathematics. SpringerVerlag, Berlin, english edition, 2002. Translated from the French by Patrick Ion and revised by the author.

[Sha64] Stephen S. Shatz. Cohomology of artinian group schemes over local fields. Ann. of Math. (2), 79:411-449, 1964

[Spa88] N. Spaltenstein. Resolutions of unbounded complexes. Compositio Math., 65(2):121-154, 1988.

[Suz13] Takashi Suzuki. Duality for local fields and sheaves on the category of fields. Preprint, arXiv:1310.4941v5, 2013. 
[Swa98] Richard G. Swan. Néron-Popescu desingularization. In Algebra and geometry (Taipei, 1995), volume 2 of Lect. Algebra Geom., pages 135-192. Int. Press, Cambridge, MA, 1998.

[SY12] Takashi Suzuki and Manabu Yoshida. A refinement of the local class field theory of Serre and Hazewinkel. In Algebraic number theory and related topics 2010, RIMS Kôkyûroku Bessatsu, B32, pages 163-191. Res. Inst. Math. Sci. (RIMS), Kyoto, 2012.

[Tat58] J. Tate. WC-groups over p-adic fields, volume 13 of Séminaire Bourbaki; 10e année: 1957/1958. Textes des conférences; Exposés 152 à 168; 2e éd. corrigée, Exposé 156. Secrétariat mathématique, Paris, 1958.

[Tem11] Michael Temkin. Relative Riemann-Zariski spaces. Israel Journal of Mathematics, 185(1):1-42, 2011.

[TT90] Robert Wayne Thomason and Thomas Trobaugh. Higher algebraic $K$-theory of schemes and of derived categories. In The Grothendieck Festschrift, Vol. III, volume 88 of Progr. Math., pages 247-435. Birkhäuser Boston, Boston, MA, 1990.

[Wer97] Annette Werner. On Grothendieck's pairing of component groups in the semistable reduction case. J. Reine Angew. Math., 486:205-215, 1997.

[Xar93] Xavier Xarles. The scheme of connected components of the Néron model of an algebraic torus. J. Reine Angew. Math., 437:167-179, 1993.

Department of Mathematics, Tokyo Institute of Technology, 2-12-1 Ookayama, MeGURO, TOKYO 152-8551, JAPAN

E-mail address: suzuki.t.aw@m.titech.ac.jp 